Jing Zeng

\title{
Sprachlernspiele im DaF-Unterricht in der VR China
}

Möglichkeiten und Herausforderungen ihres Einsatzes in Universitäten

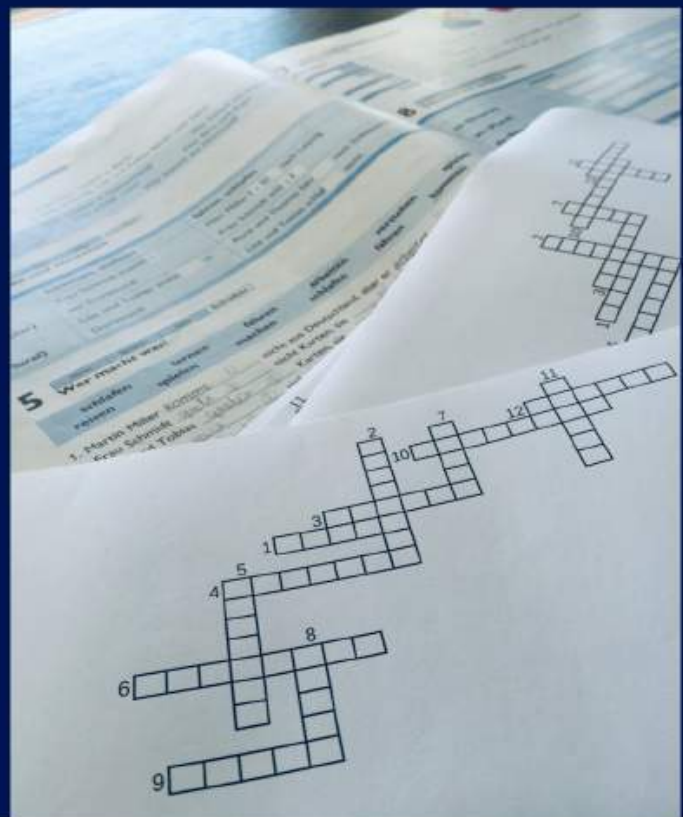


Jing Zeng

\section{Sprachlernspiele im DaF-Unterricht in der VR China}

Sprachlernspiele im DaF-Unterricht werden als multifunktionale Unterrichtsmethode für den Fremdsprachenunterricht angesehen. Ihre Wirksamkeit, insbesondere in asiatischen Ländern ist empirisch bis jetzt nur begrenzt belegt. Die Autorin stellt die Einsetzbarkeit und die Effizienz von Sprachlernspielen im DaF-Unterricht dar und versucht, ein umfassendes Bild zum Einsatz der Sprachlernspiele in China zu geben. Sie zeigt auf, dass Lehrende unter suboptimalen Rahmenbedingungen mit sorgfältiger Vorbereitung und Kreativität Sprachlernspiele als festen Unterrichtsbestandteil einsetzen können. Die Wirkung der Sprachlernspiele wird durch die Forschungsergebnisse bestätigt.

\section{Die Autorin}

Jing Zeng studierte Germanistik an der Technischen Universität Dresden und promovierte an der Technischen Universität Berlin. Sie arbeitet als DeutschDozentin an der Kunming University of Science and Technology in der Provinz Yunnan, Volksrepublik China. 
Sprachlernspiele im DaF-Unterricht in der VR China 


\section{DaZ und DaF in der Diskussion}

Herausgegeben von Jörg Meier, Beatrice Müller und Thorsten Roelcke

Begründet von Martin Löschmann

unter dem Titel

Deutsch als Fremdsprache in der Diskussion

Band 12

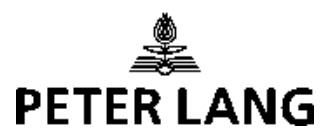




$$
\text { Jing Zeng }
$$

\section{Sprachlernspiele im \\ DaF-Unterricht in der VR China}

Möglichkeiten und Herausforderungen

ihres Einsatzes in Universitäten

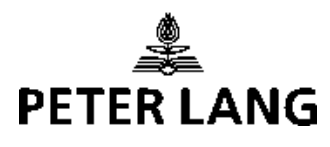




\section{Bibliografische Information der Deutschen Nationalbibliothek}

Die Deutsche Nationalbibliothek verzeichnet diese Publikation in der Deutschen Nationalbibliografie; detaillierte bibliografische

Daten sind im Internet über http://dnb.d-nb.de abrufbar.

Zugl.: Berlin, Technische Univ., Diss., 2019,

u. d. T. Möglichkeiten und Herausforderungen des Einsatzes von Sprachlernspielen im DaF-Unterricht an Universitäten in der

VR China: eine empirische Untersuchung an der Technischen Universität Kunming

Diese Publikation wurde gefördert aus dem Open-Access-Publikationsfonds der TU Berlin.

Umschlagabbildung: @ Jing Zeng

ISSN 2629-4907

ISBN 978-3-631-80063-8 (Print)

E-ISBN 978-3-631-81241-9 (E-PDF)

E-ISBN 978-3-631-81242-6 (EPUB)

E-ISBN 978-3-631-81243-3 (MOBI)

DOI $10.3726 / \mathrm{bl} 6557$

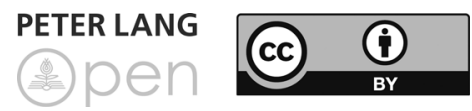

Open Access: Dieses Werk ist lizenziert unter einer Creative Commons Namensnennung 4.0 Internationalen Lizenz (CC-BY) Weitere Informationen: https://creativecommons.org/licenses/by/4.0/

Diese Publikation wurde begutachtet.

๑) Jing Zeng 2020

Peter Lang $\mathrm{GmbH}$

Internationaler Verlag der Wissenschaften

www.peterlang.com 


\section{Vorwort}

Seit dem Jahr 2011 biete ich an der Technischen Universität Kunming „Hochschuldeutsch“-Kurse für Studierende von Natur- und Technikwissenschaften an. Die Frustration, die ich in meinem Unterrichtsalltag erlebe, bildet den Anlass für die vorliegende Forschung. Die Mehrheit meiner Lernenden kommt aus ökonomisch unterentwickelten Gebieten von Südwest-China, wo ungünstige Schulbedingungen herrschen. Im Fremdsprachenunterricht sind sie schweigsam und zurückhaltend, weil sie nur negative Lernerfahrung damit gemacht haben und demotiviert sind. Um ihr Interesse für das Fremdsprachenlernen zu wecken und den Unterricht lebhafter zu gestalten, habe ich versucht, Sprachlernspiele im Unterricht einzusetzen. Nach drei Jahren intensiver Arbeit liegen die Resultate dieses Versuchs nun vor Ihnen.

An dieser Stelle möchte ich allen, die an dem guten Gelingen dieser Forschung beteiligt waren, ganz herzlich danken. Eine wissenschaftliche Arbeit ist nie das Werk einer einzelnen Person. Beteiligt waren eine Menge mir nahestehender Menschen.

An erster Stelle, muss mein Doktorvater Prof. Dr. Thorsten Roelcke genannt werden. Die Mails, die Gespräche und die wertvollen Ratschlägen von ihm haben mir immer geholfen. So eine Betreuung kann nicht als selbstverständlich angesehen werden. Auch Prof. Dr. LI Yuan bin ich für ihr zweites Gutachten zum Dank verpflichtet, die mich mit ihren Anregungen unterstützt hat. Ohne den wertvollen akademischen Rat von Prof. Dr. Thorsten Roelcke und Prof. Dr. LI Yuan wäre diese Arbeit nicht entstanden.

Ein ganz besonderer Dank gilt der Fakultät für Fremdsprachen und -kulturen der Technischen Universität Kunming, die mir Zeit und Unterstützung für die Bearbeitung der Dissertation gegeben hat. Ebenso geht mein Dank an alle Kolleginnen und Studierenden, die an dem Forschungsprojekt teilgenommen haben.

Nicht minder aufreibend waren die vergangenen Jahre für meine Familie, die dieses Werk in allen Phasen mit jeder möglichen Unterstützung bedacht haben. Meinem Mann und meinen Eltern bin ich dafür auf ewig dankbar.

Darüber hinaus gilt mein Dank auch meinen Freundinnen und Freunden in Deutschland. Silke Kärcher und ihrem Vater, Herrn Reinhard Kärcher, gebührt mein herzlichster Dank für ihre Unterstützung bei der sprachlichen Korrektur. Auch bei Florian Stoverink und seinem liebevollen Freundeskreis möchte ich mich bedanken, besonders für die Unterkunft und die stetige Unterstützung. 



\section{Inhaltsverzeichnis}

1 Einleitung

2 Deutsch als Fremdsprache in China: Entwicklung, Tendenzen und das Problemfeld vom Einsatz der interaktiven/ kommunikativen Methode

2.1 Überblick der Entwicklung und Tendenzen vom institutionellen

DaF-Unterricht in China 13

2.1.1 Der Beginn der institutionellen Fremdsprachenausbildung ......... 14

2.1.2 Die tastende Phase vom 1949 bis 1977 ............................................. 16

2.1.3 Die rasende Entwicklungsphase .......................................................... 19

2.2 Zum Einsatz der interaktiven/kommunikativen Methode im

DaF-Unterricht in China ........................................................................ 21

2.2.1 Lehrerbezogene Hindernisse ............................................................. 22

2.2.2 Lernerbezogene Hindernisse ....................................................... 28

2.2.3 Hindernisse in den Rahmenbedingungen ...................................... 31

2.2.3.1 Das Problemfeld „Großgruppenunterricht“ ..................... 33

2.2.3.2 Exkurs: Möglichkeiten des Großgruppenunterrichts ....... 36

2.2.4 Willingness to Communicate ............................................................. 39

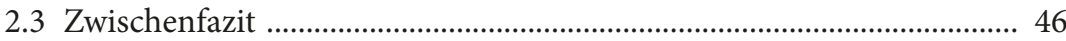

3 Sprachlernspiele: Begriff und Forschungsstand …………………. 49

3.1 Zur Definition vom Spiel im allgemeinen Sinn ..................................... 49

3.2 Zur Definition von Sprachlernspielen im Fremdsprachenunterricht ... 52

3.2.1 Die Terminologien und die Zuordnung der Sprachlernspiele ...... 53

3.2.2 Abgrenzung und Gemeinsamkeiten von Sprachlernspielen und Spiel .................................................................................... 55

3.2.3 Abgrenzung und Gemeinsamkeiten von Sprachlernspielen und anderen Arbeits- und Übungsformen ...................................... 59

3.3 Die Effekte der Sprachlernspiele 63 
3.3.1 Die Funktion von Spielen im allgemeinen Sinn .............................. 63

3.3.2 Effekte von Sprachlernspielen im Fremdsprachenunterricht ........ 65

3.3.2.1 Aspekt der Motivation ......................................................... 65

3.3.2.2 Aspekt der Kommunikation und Interaktion ..................... 66

3.3.2.3 Aspekt der Lerneffizienz und Wissensvermittlung ........... 67

3.4 Forschungsstand vom Einsatz der Sprachlernspiele ................................. 68

3.4.1 Allgemeiner Forschungsstand des Spieleinsatzes im

Fremdsprachenuntericht .............................................................. 68

3.4.2 Empirische Forschungen zum Einsatz der Sprachlernspiele im DaF-Unterricht ......................................................................... 71

3.4.2.1 Empirische Forschungen zum Einsatz der Sprachlernspiele im europäischen Raum ............................ 71

3.4.2.2 Empirische Forschungen zum Einsatz der Sprachlernspiele in Japan .................................................... 75

3.4.2.3 Empirische Forschungen zum Einsatz der Sprachlernspiele in China .................................................. 77

3.4.2.4 Schlussfolgerung zu den empirischen Forschungen .......... 78

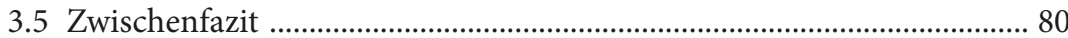

\section{Die empirische Untersuchung: Hintergrund und} Herangehensweise

4.1 Lernumfeld: Studienbegleitender Deutschunterricht an

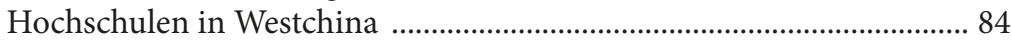

4.1.1 Studienbegleitender Deutschunterricht: Situation von „Hochschuldeutsch“ ........................................................................ 86

4.1.2 Zur Bildungsungleichheit zwischen Ost- und Westchina .............. 88

4.1.3 Zur Situation der Fremdsprachenausbildung in der Provinz

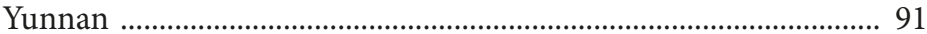

4.1.4 Schlussfolgerung zum Lernumfeld ................................................... 94

4.2 Forschungsdesign ........................................................................................ 95

4.2.1 Forschungsgegenstand ................................................................... 97

4.2.2 Organisatorischer Hintergrund und Probandensituation ............. 99 
4.2.3 Forschungsmaterialien: Lehrwerk, Spieladaption und Didaktisierung

\section{Empirische Untersuchung Teil I: Die Wirkung von} Sprachlernspielen auf Motivation und Lernfortschritt von DaF-Lernenden in China

5.1 Forschungsphase Wintersemester 2016-17 113

5.1.1 Forschungsdesign Wintersemester 2016-17

5.1.2 Auswertung und Analyse zu der Befragung zur Motivation und Unterrichtsevaluation im Wintersemester 2016-17

5.1.3 Auswertung und Analyse zu den Ergebnissen der Semesterprüfungen im Wintersemester 2016-17

5.1.4 Auswertung und Analyse zu der Befragung zum Einsatz der Sprachlernspiele im Wintersemester 2016-17

5.1.5 Schlussfolgerung der Forschungsphase Wintersemester 2016-17 126

5.2 Forschungsphase Sommersemester 2017 128

5.2.1 Forschungsdesign im Sommersemester 2017 128

5.2.2 Auswertung und Analyse zu der Befragung zur Motivation und Unterrichtsevaluation im Sommersemester 2017

5.2.3 Auswertung und Analyse zu den Ergebnissen der

Semesterprüfung im Sommersemester 2017

5.2.4 Auswertung und Analyse zu den Ergebnissen der C- Tests im Sommersemester 2017

5.2.5 Auswertung und Analyse zu der Befragung zum Einsatz der Sprachlernspiele im Sommersemester 2017

5.2.6 Auswertung und Analyse zu den Ergebnissen von Unterrichtsbeobachtungen im Sommersemester 2017 ................. 143

5.2.7 Schlussfolgerung der Forschungsphase Sommersemester 2017 .... 149

5.3 Auswertung und Analyse zum Lehrtagebuch vom Studienjahr 2016-2017 
6 Empirische Untersuchung Teil II: Praxisorientierte Anweisung vom Einsatz der Sprachlernspiele im DaF-Unterricht in China

6.1 Effiziente Planung und Durchführung von spielerischer

Gruppenarbeit im Großgruppenunterricht 163

6.2 Spieladaption: Kreativität und Adäquatheit ........................................... 168

6.3 Kriterien für bevorzugte Spieltypen ....................................................... 176

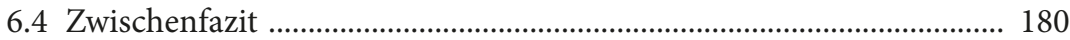

7 Fazit und Ausblick .................................................................................. 183

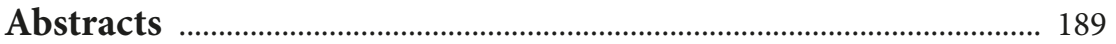

Abbildungsverzeichnis ....................................................................... 191

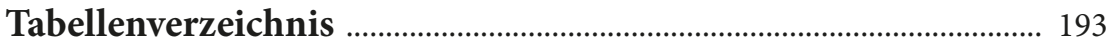

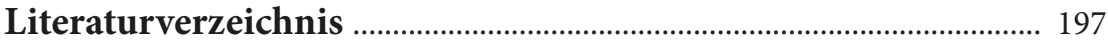

Dieses Werk enthält zusätzliche Informationen als Anhang. Sie können von unserer Website heruntergeladen werden. Die Zugangsdaten entnehmen Sie bitte der letzten Seite der Publikation. 


\section{Einleitung}

Nach Angaben des deutschen Auswärtigen Amtes (2015, S. 4) gewinnt die deutsche Sprache in den letzten Jahren immer mehr Bedeutung in China. Die mangelnde Qualität des chinesischen DaF-Unterrichts wird Mittelpunkt der Diskussion, während die Anzahl der deutschen Lernenden in China und chinesische Studierende in Deutschland in den letzten Jahren rasant steigt. Die Schweigsamkeit und fehlende Bereitschaft für Interaktion von chinesischen Deutschlernenden stellt ein Problem dar, das dringend gelöst werden soll. Die fehlende kommunikative Fähigkeit führt zur mangelhaften sprachlichen Kompetenz im Unterricht, später zu Problemen im Studium und Integrationsschwierigkeiten in Deutschland (vgl. Zeilinger 2006, S. 11f.). Eine Verbesserung der Unterrichtsqualität ist erforderlich.

Sprachlernspiele werden als Unterrichtsmethode seit der kommunikativen Wende in den 70er Jahren in Deutschland weitgehend akzeptiert. Sprachlernspiele können Interaktion und Kommunikation im Unterricht fördern sowie Motivation der Lernenden verbessern. Diese Methode wird aber in Fernost, wo eine andere Lehr- und Lerntradition herrscht, bisher nicht systematisch angewendet und erforscht. Auch in deutschsprachigen Ländern wird die Wirkung dieser Methode nur ungenügend empirisch belegt.

Vor diesem Hintergrund beschäftigt sich diese Arbeit mit folgender zentraler Fragestellung: In wieweit können Sprachlernspiele auf Motivation und Lernfortschritt der Lernenden wirken? Von besonderem Interesse ist eben die Frage, wie man Sprachlernspiele im DaF-Unterricht in China einsetzen kann, sodass die in Europa geborene Methode für die chinesischen Rahmenbedingungen adaptiert werden kann. Die vorliegende Arbeit befasst sich nämlich mit Möglichkeiten und Herausforderungen vom Einsatz der Sprachlernspiele im DaF-Unterricht in China mit Hilfe einer empirischen Untersuchung.

Um die genannten Fragen zu beantworten, beginnen wir mit einer ausgiebigen Betrachtung von methodischen Tendenzen des Fremdsprachenunterrichts in China (siehe Kapitel 2). In diesem Kapitel sollen der Einsatz und die Akzep$\operatorname{tanz}$ der interaktiven/kommunikativen Methode ${ }^{1}$ im DaF-Unterricht herauskristallisiert werden. Die Schwierigkeiten des Einsatzes dieser Methode werden

1 Da interaktiver Unterricht „ die gesamte Sprech- und Lernsituation“ bezeichnet, während kommunikativer Unterricht „sich auf die Fremdsprachen-Sprechsituationen bezieht“(Schiffler 1985, S. 13f.), wird in der vorliegenden Arbeit mit Berücksichtigung auf Sprachlernspiel die Bezeichnung “interaktive / kommunikative Methode” benutzt. 
aus der Perspektive der Lehrenden und der Lernenden veranschaulicht, unter Berücksichtigung der Rahmenbedingungen des chinesischen DaF-Unterrichts.

Anschließend soll der Begriff Sprachlernspiel im Kapitel 3 näher betrachtet werden. Die Definition und der Forschungsstand der Sprachlernspiele im Fremdsprachenunterricht werden diskutiert. Die erwarteten Effekte von Sprachlernspielen werden eben hier dargestellt.

Eine einjährige Aktionsforschung wird durchgeführt, um die Einsetzbarkeit und Wirkung von Sprachlernspielen im DaF-Unterricht in China zu überprüfen. Ein Schwerpunkt der Forschung wird bei der Wirksamkeit von Sprachlernspielen auf Motivation und Lernfortschritt von Lernenden gesetzt. Befragungen, Tests, Unterrichtsbeobachtungen und Lehrtagebücher werden qualitativ und quantitativ analysiert, um die Effizienz von Sprachlernspielen zu kristallisieren. Das Design, das Umfeld und die Methodik der empirischen Forschung wird im Kapitel 4 dargestellt. Das Kapitel 5 konzentriert sich auf die Forschungsergebnisse zur Wirkung von Sprachlernspielen auf die Motivation- und Leistungsebene, während das Kapitel 6 über praxisorientierte Anweisung für den Spieleinsatz im DaF-Unterricht in China Auskunft gibt. Schließlich werden die wichtigsten Forschungsergebnisse und ein relevanter Ausblick im Kapitel 7 veranschaulicht.

Sprachlernspiel handelt nämlich nicht nur um Sprechen, sondern von allen Interaktivonsformen im Unterricht. 


\section{Deutsch als Fremdsprache in China: Entwicklung, Tendenzen und das Problemfeld vom Einsatz der interaktiven/ kommunikativen Methode}

In diesem Kapitel wird zunächst die Entwicklung des institutionellen DaFUnterrichts seit Anfang des modernen Bildungssystems der VR China bis heute skizziert, um die allgemeine Information zum DaF-Unterricht in China zu liefern. Die historischen politischen Rahmenbedingungen und ihre Beziehungen mit verschiedenen Entwicklungsphasen der Fremdsprachenausbildung werden dargestellt, um die didaktischen und methodischen Tendenzen der jeweiligen Zeit besser verstehen zu können.

Danach wird die Einsatzsituation der kommunikativen Methode im DaFUnterricht in China in den Blick genommen. Die Schwierigkeiten des Einsatzes der kommunikativen Methoden werden unter die Lupe genommen: aus der Perspektive von Lehrenden und Lernenden unter Berücksichtigung soziokultureller Besonderheiten und organisatorischer Hindernisse. Das Konzept „Willingness To Communicate“ (WTC) wird anschließend vorgestellt, um ein vertieftes Verständnis zum passiven Unterrichtverhalten der chinesischen Lernenden zu liefern und Verbesserungsmöglichkeiten zu finden.

\section{1 Überblick der Entwicklung und Tendenzen vom institutionellen DaF-Unterricht in China}

Die Entwicklung des institutionellen DaF-Unterrichts widerspiegelt die politische und soziale Lage von China in den letzten 150 Jahren. Viele soziopolitischen Faktoren spielen dabei eine Rolle und entscheiden, wo, wie und welche Fremdsprache gelehrt und gelernt wird. Die Entwicklung vom DaF-Unterricht in China zeigt die chinesische Geschichte in den vergangenen 150 Jahren, die von vielen komplizierten Faktoren verwickelt beeinflusst wird, wie von Kriegen, von der Umstrukturierung des Landes, von stets veränderten internationalen politischen Beziehungen und der wirtschaftlichen Entwicklung des Landes.

Thelen (2003, S. 54) ist der Meinung, dass es große Unterschiede vom Verständnis der didaktischen Methoden im Fremdsprachenunterricht zwischen Deutschland und China gibt, obwohl die Begriffsbezeichnungen identisch sind. Die historischen, kulturellen und sozialen Gegebenheiten müssen berücksichtigt 
werden, um die Unterschiede nachvollziehbar darzustellen. Deswegen ist es für die vorliegende Arbeit von Bedeutung, den chinesischen DaF-Unterricht unter den historischen, kulturellen und sozialen Rahmenbedingungen zu betrachten, um die Tendenzen der didaktischen Methoden und die Entwicklung vom Fremdsprachenunterricht in China besser zu verstehen.

Die vorliegende Diskussion stützt sich hauptsächlich auf die Arbeit von Li (2012), Wei (2009) und Zhu (2009), sie betrachtet die Entwicklung des institutionellen Fremdsprachenunterrichts des modernen China in 3 Hauptetappen: der Beginn der institutionellen Fremdsprachenausbildung von der späten Qing-Dynastie bis zur Gründung der VR China im Jahr 1949, die tastende Phase von 1949 bis 1977 und die rasante Entwicklungsphase von 1977 bis heute.

\subsubsection{Der Beginn der institutionellen Fremdsprachenausbildung}

Es ist heutzutage anerkannt, dass die fremdsprachliche Ausbildung den Anfang des modernen chinesischen Bildungswesens bezeichnet (vgl. Li und Xu 2007, S. 1). Li (2012, S. 69) schließt sich dieser Meinung an und findet, dass die fremdsprachliche Ausbildung eine bedeutende Rolle bei der modernen Transformation des chinesischen Bildungswesens spielt.

Über den Beginn des Fremdsprachenunterrichtes der modernen Zeit wurde bereits sehr viel geschrieben. Der Anfang des Fremdsprachenunterrichts kann bis in die späte Qing-Dynastie (1840-1911) zurückverfolgt werden (vgl. Li 2012, S. 69; Zhu 2019, S. 8; Hernig 2010, S. 1637; Steinmüller 2019, S. 228). Tongwenguan, gegründet im Jahr 1862 in Beijing, ist die erste institutionelle Fremdsprachenausbildungsanstalt von China und gibt Deutschunterricht seit dem Jahr 1871. Das Ziel von Tongwenguan ist, Dolmetscher und Übersetzer mit diplomatischer Orientierung für das damalige kaiserliche China auszubilden, später auch in Kombination mit naturwissenschaftlichen Fächern (vgl. Hernig 2010, S. 1637; Li 2012, 70,77; Zhao 2019, S. 20).

Ähnliche „(kaiserliche) Fremdsprachenhochschulen“ sind später in anderen Städten gegründet worden (vgl. Hernig 2010, S. 1637). Die ausgewählten Fremdsprachen vor Ort sind mit den regionalen politischen und wirtschaftlichen Beziehungen mit dem Ausland stark abhängig: Deutsch wird beispielsweise in Beijing und Shanghai angeboten (vgl. Li 2012, S. 77). Auch militärische Hochschulen in Tianjin, Guangdong und Hubei bieten Deutschkurse an. Seit 1903 können Studierende an chinesischen Hochschulen Deutsch als zweite Fremdsprache lernen (vgl. Wei 2009, S. 270).

Erst im Jahr 1904 entsteht das erste moderne Bildungssystem in China. Die kaiserliche Beamtenprüfung wird im Jahr 1905 abgeschafft. Im Jahr 1912 wurde 
die feudalistische Kaiserherrschaft in China durch die Xinhai-Revolution im Jahr 1911 beendet (vgl. Li 2012, S. 71). Universitäten wie Tongji bieten seit 1907 Deutschkurse für Studierende von medizinischen und technischen Fächern an (vgl. Wei 2009, S. 271). Die Stellung der westlichen Philosophie steigt mit dem wachsenden Interesse an dem westlich-orientierten Wertesystem und der modernen Gesellschaft (vgl. Hernig 2010, S. 1637). Die Universität Beijing, Universität Fudan, Universität Shanghai gründen nacheinander deutsche Abteilungen (vgl. ebenda, S. 1637; Wei 2009, S. 271). Das ist die erste Boom-Entwicklung des Deutschunterrichts in China, die aber wegen der Invasion der japanischen Armee und des Einbruchs vom zweiten Weltkrieg wieder zum Stillstand gekommen ist (vgl. Steinmüller 2019, S. 228).

Der Fremdsprachenunterricht dieser Phase hat folgende Merkmale:

1. Die ersten Fremdsprachenhochschulen haben klare Bildungsziele. Die Zielgruppen sind ausgewählte Personen mit gutem Ausbildungshintergrund, die sich fremdsprachlich weiterbilden lassen und später für die Entwicklung des Landes beruflich beschäftigt werden sollen. Das sind Dolmetscher oder Übersetzungspersonal mit diplomatischen Eigenschaften, oder naturwissenschaftliche und technische Studierenden, die moderne ausländische Fortschritte für die Entwicklung des Landes erlernen. Es ist also keine Bildung für normale Bürger, sondern für die Elite der Gesellschaft (vgl. Li 2012, 95,97; Zhao 2019, S. 23; Li und Lian 2017, S. 116).

2. Mangel an gut ausgebildeten Lehrenden. Die Lehrkräfte von Fremdsprachenhochschulen wie Tongwenguan sind Muttersprachler und Chinesen, die in zielsprachigen Ländern studiert haben. Allerdings zeigt sich der Mangel an Lehrenden langsam, während sich der Fremdsprachenunterricht national weiter entwickelte (vgl. Li 2012, S. 95). Wie Reinbothe (2007, S. 59-67) berichtet, fehlte es für technische und naturwissenschaftliche Fächer an den damaligen Hochschulen an passenden Lehrkräften: Die deutschen Lehrenden, die Fachunterricht geben, konnten kein Chinesisch. Die Studierenden waren sprachlich und fachlich unausgebildet, um die Lehrveranstaltung zu verstehen. Deswegen kann man feststellen, dass die Qualität des Fremdsprachenunterrichts hinsichtlich des Bedarfs des Landes unbefriedigend war.

Zur häufig eingesetzten didaktischen Methode in dieser Zeit vertreten die Forschenden abweichende Positionen. Zhao (2019, S. 23) ist der Meinung, dass die „Direkte Methode“ eine Hauptrolle in dieser Phase spielte, während andere Autoren meinen, dass die Grammatik-Übersetzungsmethode am Anfang des 20. Jhd. am meisten eingesetzt wurde: Hess (1992, S. 70f.) gibt einen Überblick zur Didaktik jener Zeit und meint, dass die „Direkte Methode“ oft an 
Missionarsschulen eingesetzt wurde, weil die ausländische Lehrkraft dort nur ihre Muttersprache beherrschte. An den meisten Schulen, an denen man kaum Kontakt mit Ausländern hatte, spielte die Grammatik-Übersetzungsmethode die Hauptrolle. Li (2012, S. 96) begründet, dass die Hauptbildungsziele die Fertigkeiten Lesen und Übersetzen waren und die Grammatik-Übersetzungsmethode diesen Zielen entsprach. Außerdem deutet sie an, dass die direkte Methode von manchen Lehrenden befürwortet und erforscht, aber nicht sehr weit verbreitet eingesetzt wurde. Thelen (2003, S. 55-57) stimmt Mitschian (1991, S. 260), zu dass die Grammatik-Übersetzungsmethode von den im Ausland studierten Chinesen zurückgebracht wurde, weil diese Methode damals im Western dominierend war. Da die deutsche Abteilung an Universitäten literarisch-philosophisch orientiert war, wurde dort auch die Grammatik-Übersetzungsmethode eingesetzt. Die „Direkte Methode“ trat erst in den 30en Jahren des letzten Jahrhunderts ein und übte nur geringen Einfluss aus (vgl. ebenda, S. 261).

\subsubsection{Die tastende Phase vom 1949 bis 1977}

Seit der Gründung der VR China gewinnt Bildung eine wachsende Bedeutung. Es wird versucht, das Bildungssystem des alten China zu verändern (vgl. Li 2012, S. 98; Wei 2009, S. 272). Allerdings erlebte die Fremdsprachenausbildung in dieser Phase eine Reihe von Schwierigkeiten wegen politischer und sozialer Umwandlungen. Li (2012, S. 98) ist der Meinung, dass die fremdsprachliche Ausbildung dieser Zeit wie ein Kind ist, das gerade Laufen lernt. Politik spielt in dieser Phase eine besonders wichtige Rolle (vgl. Hess 1992, S. 71).

Da in den 1950er Jahren der Aufbau des Staates unter starkem Einfluss der damaligen UdSSR ist, trägt das Bildungssystem damaliger Zeit signifikante Merkmale von dem der UdSSR (vgl. Li 2012, S. 98; Wei 2009, S. 272; Hernig 2010, S. 1638). Die fremdsprachlichen Fakultäten an Universitäten werden mehrheitlich abgeschafft, während sich Fremdsprachenhochschulen entwickeln (vgl. Wei 2009, S. 272). Russisch wird die führende Fremdsprache an Schulen und anderen Bildungsinstitutionen, während andere Fremdsprachen wie Deutsch oder Englisch in Vergessenheit geraten (vgl. Li 2012, S. 98; Thelen 2003, S. 57; Hernig 2010, S. 1638; Li und Lian 2017, S. 117). Die Fremdsprachenhochschule Beijing, die Universität Beijing, die Universität Nanjing und die Universität Shanghai gründen die ersten Deutschabteilungen (das Fach Germanistik) in dieser Zeit (vgl. Wei 2009, S. 272-274; Hernig 2010, S. 1638; Kong 2007, S. 123). Nach der Angabe von Wei (2009, S. 274) gab es zwischen 1953-1956 insgesamt 138 Germanistik-Absolvierenden von den vier genannten Universitäten. Der 
studienbegleitende Deutschunterricht an Universitäten war zu dieser Zeit in Stillstand geraten (vgl. Zhu 2009, S. 628).

Der Wendepunkt kommt im Jahr 1956. Das Bildungsministerium erkannte den Nachteil der führenden Rolle von Russisch und entschied, die Ausbildung von anderen Fremdsprachen (hauptsächlich Englisch) zu entwickeln (vgl. Li 2012, S. 99; Hu 2001, S. 247). Bis zum Jahre 1966 gründeten weitere 12 Universitäten sowie Hochschulen eine Deutschabteilung. Im Jahr 1959 dürfen die Universitäten je nach dem Bedarf und der Situation von Lehrkräften eine zweite Fremdsprache wie Deutsch, Japanisch und Französisch für Studierende anbieten (vgl. Wei 2009, S. 275).

In den 60er Jahren erlässt der Staat eine Reihe von politischen Richtlinien für die Restrukturierung der Fremdsprachenausbildung wegen des Zusammenbruchs der chinesisch-sowjetischen Beziehung. Der Mangel von Fremdsprachenpersonal ist nach Angabe von $\mathrm{Hu}$ (2001, S. 248) wegen der einseitigen Russischausbildung nicht zu vernachlässigen: zwischen den Jahren 1964 bis 1966 gab es nationalweit 450 Germanistikabsolvierende, während der Staat 842 brauchte.

Im Jahr 1964 bildete sich die staatliche Planungsabteilung für Fremdsprachen und führte einen siebenjährigen Entwicklungsplan der Fremdsprachenausbildung aus. Der Plan fördert die Gründung von weiteren Fremdsprachenhochschulen und den Aufbau von fachlichem und studienbegleitendem Fremdsprachenunterricht. Dieser Plan enthält zahlreiche Vorschläge, die für die Entwicklung des Fremdsprachenunterrichts von Bedeutung sind, wird allerdings wegen der Kulturrevolution (1966-1976) nicht vollständig umgesetzt (vgl. Hu 2001, S. 248f.; Li 2012, S. 101; Li und Lian 2017, S. 118).

Die Kulturrevolution hat katastrophale Konsequenzen für die chinesische Bildung. Die Fremdsprachenausbildung ist besonders stark betroffen (vgl. Li und Lian 2017, S. 118), besonders in der ersten Phase zwischen Jahr 1966 bis 1970. Lehraktivitäten und wissenschaftliche Forschung kamen zum Erliegen, weil die meisten Intellektuellen zur körperlichen Arbeit auf das Land geschickt werden (vgl. Wei 2009, S. 275; Hernig 2010, S. 1638). Fremdsprachliche Kenntnisse können als Zeichen für "Spionage für das Ausland“ (Li und Lian 2017, S. 119) betrachtet werden. Ausländische Einflüsse wie Zeitungen, Zeitschriften, Bücher sowie die Lehrtätigkeit ausländischer Lehrenden werden untergesagt. Es herrschte eine chaotische Unordnung an Bildungsinstitutionen (vgl. Li 2012, S. 101). Die Situation verbessert sich erst im Jahr 1970 (vgl. Li 2012, S. 102; Hernig 2010, S. 1638; Wei 2009, S. 276f.; Thelen 2003, S. 60-62). Die Fremdsprachenausbildung gewinnt seitdem immer mehr an Bedeutung. 
Zusammenfassend lässt sich sagen, dass die fremdsprachliche Ausbildung zu diesem Zeitraum immer wieder Fort- und Rückschritte erlebt hat. Das Schicksal der fremdsprachlichen Fachleute dieser Zeit hängt extrem mit der nationalen politischen und sozialen Lage zusammen. In den 50er Jahren, müssen viele Englisch-Lehrende an der Schule wegen der einseitigen Betonung von Russisch eine neue Fremdsprache lernen und gleichzeitig lehren. Manche Lehrenden gaben ihre Lehrtätigkeit deswegen auf (vgl. Li 2012, S. 99; Hu 2001, S. 247). Li (2012, S. 107) schreibt dazu:

Es fehlt Lehrpersonal... in der damaligen Lage ist es schon problematisch, genügende Lehrenden einzustellen. Über gut qualifizierte Lehrende kann man schon vergessen (nationalperspektiv gesehen $)^{2}$.

Danach folgt die Kulturrevolution, die das Leben und die Karriere vieler Fremdsprachenlehrenden beinahe zerstört hat (vgl. Li 2009, S. 149). Es ist kein Wunder, dass sich der Fremdsprachenunterricht der betreffenden Zeit didaktisch und methodisch nicht entwickelt.

Zwischen 1949 und 1966 wird die Grammatik-Übersetzungsmethode weiter beibehalten (vgl. Li 2012, S. 109; Thelen 2003, S. 57). Die eingeführten Lehrmaterialien sowie die Methode der entsandten Experten aus der Sowjetunion und der DDR entsprechen auch dieser Methode (vgl. Hess 1992, S. 71; Thelen 2003, S. 57). Da die diplomatische Beziehung zur DDR nach der Gründung der VR China aufgenommen wird, bietet das Herder-Institut zum Anfang der 1950 Fortbildungskurse an und schickt deutsche Experten nach China. Laut Zhao (2019, S. 23f.) übte das Herder-Institut auf den DaF-Unterricht in China starken Einfluss aus, der bis heute noch zu sehen ist. Ein anderer ausländischer methodischer Einfluss dieser Phase ist die audiolinguale Methode (vgl. Thelen 2003, S. 58; Zhao 2019, S. 24; Wei 2009, S. 296). Das Sprachlabor wird eingeführt und spielt bis heute immer noch eine Rolle an vielen Universitäten.

Trotz verschiedener Einflüsse aus westlichen Ländern kann man wegen der politischen und sozialen Lage der betreffenden Zeit nicht viel Änderung erzeugen. Li (2012, S. 116) deutet an, dass die Grammatik-Übersetzungsmethode im schulischen Englischunterricht die Hauptrolle spielt, weil die Lernziele des damaligen Fremdsprachenunterrichts Lesen und Übersetzen waren. Die ausländischen Konzepte werden oberflächlich aufgenommen. Wei und Qin (2017, S. 146) sind der Meinung, dass sich die Bildungsziele von Fremdsprachenhochschule an Dolmetscher und Übersetzungspersonal richten, während

2 Das chinesische Original dieses Werks habe ich selbst ins Deutsche übersetzt. Das gilt für andere direkte Zitationen von diesem Werk in der vorliegenden Arbeit. 
an Universitäten Literaturwissenschaft studiert wird. Die Grammatik-Übersetzungsmethode scheint deswegen zielentsprechend zu sein. Mitschian (1991, S. 263) teilt zum DaF-Unterricht am Ende 1980 und Anfang 1990 eben ähnliche Position wie Li (2012):

Wie aktuelle Unterrichtsbeispiele zeigen, verbirgt sich heute hinter der Bezeichnung „audiolingualer" Unterricht häufig nur der Einsatz des Sprachlabors oder eines Tonbandgeräts, ohne dass der Unterrichtsgang dadurch wesentlich verändert hätte.

Hess (1992, S. 72f.) erläutert, dass die audiolinguale Methode eine Umsetzung der Lernziele erzeugt hat, aber leider nur an führenden Universitäten praktiziert wurde, während der größte Teil der Unterrichtspraxis von der GrammatikÜbersetzungsmethode geprägt wurde. Auch im heutigen Sprachunterricht im Sprachlabor kann man dem Phänomen immer wieder begegnen. Die Lernmaterialien sind wahrscheinlich interkulturell und kommunikativ, aber der Unterrichtsgang kann gleich wie damals sein (nähere Erläuterung im Kapitel 2.2.3). Laut Mitschian (1991, S. 263) spielte die Grammatik-Übersetzungsmethode zur damaligen Zeit die absolut dominierende Rolle, trotz der Einflüsse aus dem Ausland. Das wird in folgenden Kapiteln ausführlicher behandelt.

\subsubsection{Die rasende Entwicklungsphase}

Seit dem Jahr 1978 tritt die Fremdsprachenausbildung in die richtige Boom-Ära ein (vgl. Hu 2001, S. 250; Zhao 2019, S. 21). Im Jahr 1978 fand die Konferenz über Fremdsprachenlernen und -lehren auf staatlicher Ebene in Beijing statt, an der alle fremdsprachlichen Abteilungen der Universitäten bzw. Hochschulen in China teilnahmen. Diese Konferenz kennzeichnet den Anfang der Blütezeit der Fremdsprachenausbildung der VR China (vgl. Li 2012, S. 119; Li und Lian 2017, S. 119). Zhu (2019, S. 8) schreibt dazu:

Nach der Kulturrevolution, besonders seit der Reform- und Öffnungspolitik Chinas im Jahre 1978, verbesserte sich das gesellschaftliche Umfeld für die Fremdsprachenlehre, darunter auch Germanistik oder Deutsch als Fremdsprache, als Fach in China.

Seit Anfang der 80er Jahre gewinnt die deutsche Sprache wieder eine feste Stellung an Universitäten und Hochschulen (vgl. Wei 2009, S. 278; Hernig 2010, S. 1638). Laut einer nationalen Statistik des Bildungsministeriums im Jahr 1995 bieten 116 Universitäten bzw. Hochschulen studienbegleitenden Deutschunterricht an, der von 16460 Studierenden besucht wird (vgl. Zhu 2009, S. 642). Anfang der 80en Jahren gibt es ca. 21 Universitäten sowie Hochschulen mit dem Fach Germanistik (vgl. Wei 2009, S. 279f.; Kong 2007, S. 124). Seit Beginn des 21. Jahrhunderts gewinnt die Fremdsprachenausbildung in China immer mehr 
an Bedeutung wegen zunehmender internationaler Zusammenarbeit und vertiefender Öffnungspolitik (vgl. Li und Lian 2017, S. 120). Die Zahl der Germanistik-Institute steigt von 28 im Jahr 2000 (vgl. ebenda, S. 120) bis zu 50 bis Ende 2005 (vgl. Kong 2007, 139-140). Im Jahr 2017 gibt es schon ca. 110 GermanistikInstitute in China (vgl. Wei und Qin 2017, S. 148).

Zur Anzahl des deutschen Sprachlernens deutet Gerbig (2007, S. 317) an, dass die Zahlen der Deutschlernenden in Megastädten stetig steigt. Laut der Statistik des deutschen Auswärtigen Amtes (2015, S. 7) gehört China zu den Ländern, wo sich die Zahlen der Lernenden sehr dynamisch entwickeln: „Allein im Hochschulbereich sind die absoluten Zahlen seit der letzten Erhebung im Jahr 2010 um 24 \% gestiegen“(Auswärtiges Amt 2015, S. 29). Die Statistik zeigt, dass die deutsche Sprache in den letzten 20 Jahren in China nationalweit verbreitet ist:

Tabelle 1: Ausgewählte Daten zur Situation Deutschlernen in China 2015 (vgl. Auswärtiges Amt 2015, S. 12)

Gesamtzahl der Deutschlernenden

117.487

(Schulen, Universitätsbereich und Erwachsenenbildung)

Anzahl der Hochschulen mit Deutschunterricht

327

Anzahl der Deutsch-lernenden Studierenden

44.945

Zuwachs der Gesamtzahl im Vergleich zu 2010

$+8.945$

Während die Lerneranzahl nationalweit deutlich gestiegen ist, kann man allerdings über die Didaktik und Methodik des Deutschunterrichts seit dem Jahr 1978 bis heute keine eindeutige positive Änderung sehen. Lehrerzentrierter Frontalunterricht, Auswendiglernen und die Dominanz der Grammatik-Übersetzungsmethode sind Unterrichtsalltag (vgl. Zeilinger 2006, S. 7-9; Thelen 2003, S. 242).

Seit der Öffnungspolitik gegen Ende der 70en Jahren gibt es immer mehr Zusammenarbeit zwischen China und den deutschsprachigen Ländern. Goldberg $(2010,3)$ weist darauf hin, dass die didaktische und methodische Entwicklung im Ausland in China großem Interesse begegnet. DAAD und Goethe-Institut arbeiten mit den Bildungsinstitutionen zusammen. Lektorenprogramme und Ausbildungen für chinesische DaF-Lehrende von DAAD spielen seit mehr als 30 Jahren bei der Verbreitung der deutschen Sprache und Verbesserung der Unterrichtsqualität eine der herausragende Rolle (vgl. Goldberger 2010, 3; Wei 2009, S. 304f.).

Zur Entwicklung der Unterrichtsdidaktik äußert sich Wei (2009, S. 296f.): in den 80er Jahren wird die kommunikative Methode in China bekannt und 
gewinnt immer mehr an Bedeutung, während die traditionelle GrammatikÜbersetzungsmethode gleichzeitig eine feste Stelle im Unterricht behält.

Um die Unterrichtsqualität zu verbessern, gibt es auch zahlreiche Bemühungen von dem chinesischen Deutschkreis auf staatlicher Ebene: Überarbeitungen von Curricula für Germanistik und Studienbegleitendes Deutsch werden in den letzten 30 Jahren mehrmals durchgeführt (vgl. Zhu 2019, S. 12f.; Wei 2009, S. 287-289; Kong 2007, S. 125f.). Fachtagungen zum Thema Didaktik und Methodik werden organisiert und Lehrwerke werden entwickelt (vgl. Wei 2009, S. 297-300; Zhu 2009, S. 631-654).

Allerdings zeigt die Unterrichtswirklichkeit in China trotz der Bemühung chinesischer Seite und der ausländischen Mittlerorganisationen keine eindeutige Verbesserung. Kritik an der Unterrichtsqualität kann man in vielen Berichten der DAAD-Lektoren finden (vgl. Steinmüller 2019, S. 229). Eine empirische Untersuchung der jüngsten Zeit (vgl. Tian 2017) verdeutlicht auch methodische Mängel des DaF-Unterrichts an chinesischen Universitäten. Die Passivität im Unterricht ist bis heute ein signifikantes Merkmal von chinesischen Lernenden (vgl. Casper-Hehne 2015, 162).

Für interaktiven/kommunikativen Fremdsprachenunterricht und lernerzentrierte Methoden kann bis heute kein fester Platz in China und vielen anderen ostasiatischen Ländern gefunden werden, während die Grammatik-Übersetzungsmethode in diesen Ländern weit verbreitet eingesetzt wird (vgl. Chou 2015, S. 34). Thelen (2003, S. 67) ist der Meinung, dass es einen großen Unterschied zwischen Deutschland und China bezüglich der kommunikativen Methode gibt, weil es unterschiedliche wissenschaftliche Traditionen sowie institutionelle Rahmenbedingungen gibt. Das Verstehen der Interaktionsschwierigkeiten im Unterricht kann dazu beitragen, die Situation und Probleme vom DaF-Unterricht in China konkret zu beobachten. Im Folgenden wird dieses Problemfeld unter die Lupe genommen und sich damit auseinandergesetzt, um die mangelhafte Unterrichtspraxis bezüglicher der interaktiven/kommunikativen Methode zu verstehen.

\subsection{Zum Einsatz der interaktiven/kommunikativen Methode im DaF-Unterricht in China}

Wegen der Reform und Öffnungspolitik seit Ende der 70er Jahre des letzten Jahrhunderts gewinnt die kommunikative Kompetenz von Sprachlernenden wachsende Bedeutung in China. Auf dem Arbeitsmarkt ist erhöhter Bedarf von Fachkräften mit kommunikationsfähigen Fremdsprachenkenntnissen zu beobachten. Die Defizite der mündlichen kommunikativen Fähigkeiten von 
chinesischen Fremdsprachenlernenden können deswegen nicht vernachlässigt werden. Auch die Studierenden zeigen mehr Interesse dafür, eigene Kommunikationskompetenz auf Fremdsprachen zu verbessern, weil sie bessere Berufschancen haben möchten oder Fortbildungsmöglichkeiten in Zielsprachländern suchen. Dieses gestiegene Interesse bringt eine Unzufriedenheit gegenüber der traditionellen Unterrichtsmethode mit sich (vgl. Wang 2007, S. 79-82; Thelen 2003, S. 241f.). Allerdings trifft die Methodenänderung auf eine Reihe von Schwierigkeiten.

Hier wird das Problemfeld vom Einsatz der interaktiven/kommunikativen Methoden im chinesischen DaF-Unterricht näher betrachtet. Es gibt unterschiedliche Faktoren, die den DaF-Unterricht beeinflussen können. Die von chinesischen und deutschen DaF-Lehrenden oft diskutierten soziokulturellen Faktoren, institutionelle Rahmenbedingungen und andere bezügliche Probleme werden hier tiefer untersucht. Das Konzept Willingness to Communicate wird vorgestellt, um die vorhandene Problematik besser zu verstehen.

\subsubsection{Lehrerbezogene Hindernisse}

Ein Unterricht besteht aus zwei Gruppen: die Lehrenden und die Lernenden. Die beiden Gruppen nehmen aktiv am Unterrichtsgeschehen teil und bilden mit- und untereinander Interaktion. In diesem Unterkapitel wird die Ansicht von Lehrenden in China näher betrachtet, um die Schwierigkeiten beim Einsatz der interaktiven/kommunikativen Methode aus Lehrerperspektive zu analysieren.

Das traditionelle chinesische Fremdsprachenunterrichtsmodell legt großen Wert auf Lesen und Grammatik, was den Bedarf der Lernenden und den Markt der heutigen Zeit nicht mehr trifft. Diese Tatsache wird seit langem erkannt (vgl. Wang 2007, S. 82; Wei 2009, S. 297). Wegen der Diskrepanz zwischen Bedarf und Unterricht werden Reformen, Diskussionen und Verbesserungsversuche auf didaktischer Ebene durchgeführt (vgl. Wang 2007, S. 92-103; Zhu 2009, S. 648-654). Aber bis heute hält die lehrerzentrierte Grammatik-Übersetzungsmethode immer noch ihren festen Platz im chinesischen Fremdsprachenunterricht (vgl. Tian 2017, S. 134; Chou 2015, S. 34; Li und Lian 2017, S. 138; Zeilinger 2006, S. 8f.).

Wang (2007, S. 92-103) gibt einen Überblick über die Methodendiskussionen über kommunikative Methoden von chinesischen Fremdsprachenlehrenden seit den 80er Jahren bis zum Anfang des 21. Jhd. und meint, dass in den 80er Jahren man sich auf die Frage konzentriert: Welche Methode ist für chinesische Lernende geeignet? Zur kommunikativen Methode machen sich manche Lehrende 
Sorgen um die Überbetonung von Kommunikation und die Vernachlässigung von fundamentalem Sprachwissen und schriftlicher Fähigkeit (vgl. Wang 2007, S. 93; Liang 1999, S. 203).

Wang (2007, S. 93f.) ist der Meinung, dass die Betonung des Sprachfundaments bis heute in China eine wichtige Rolle spielt. Das kann dazu führen, dass kommunikative Übungen von Lehrenden und auch Lernenden als Zeitvergeudung betrachtet werden. Man bezweifelt die Übertragbarkeit der kommunikativen Methode. Manche Lehrende sind der Meinung, dass der enorme sprachliche Unterschied zwischen Deutsch und Chinesisch eine unüberwindliche Hürde für chinesische Deutschlernende darstellt, die nur mit festem Wissen von Grammatik und Wortschatz bewältigt werden kann (vgl. Wang 2007, S. 94f.; Hess 1992, S. 382-389; Chou 2015, S. 134). Wang (2007, S. 96) kritisiert den Diskussionszustand der 80er Jahre, weil dabei viele wichtige Probleme vom Einsatz der kommunikativen Methoden vernachlässigt werden: Es wurde nicht einmal genau gefragt, was kommunikative Kompetenz bedeutet.

Zur Diskussion über die Qualität des chinesischen Fremdsprachenunterrichts wird ein Problem immer wieder erwähnt: Fehlende Lehrerausbildung. Dieses Problem wird von vielen Forschenden konkretisiert (vgl. Li und Lian 2017, S. 139f.; Wang 2007, S. 102; Steinmüller 2019, S. 232f.).

Es gibt in China bis jetzt noch keine systematische Deutschlehrerausbildung (vgl. Steinmüller 2019, S. 232; Zhu 2019, S. 8f.; Goldberger 2010, 6). In China gibt es keine Trennung von Deutsch als Fremdsprache und Germanistik (vgl. Hess 2001, S. 1584; Hernig 2010, S. 1638). Laut eines Forschungsberichts aus dem Jahr 1992 bekannten 62 \% der befragten Lehrenden, dass sie Didaktik und Methodik nie systematisch gelernt haben. $42.3 \%$ Lehrende sind davon überzeugt, dass sie ihre eigene Unterrichtsmethode dringend verbessern müssen (vgl. Wei 2009, S. 297).

Trotz dieser Erkenntnis gibt es bis heute immer noch kein strenges Germanistikstudium mit Fachrichtung DaF, obwohl die Lehrtätigkeit eine Hauptberufsauswahl für viele Germanistikstudierenden ist (vgl. Zhu 2019, S. 8f.). Laut Li und Lian (2017, S. 137) wird DaF heute nur „an einigen wenigen Universitäten als Modul angeboten".

Kong (2007, S. 128) zeigt mit einer nationalweiten empirischen Untersuchung aus dem Jahr 2004, dass Germanistik an chinesischen Hochschulen in vier Gruppen geteilt ist:

1. Traditionelle Germanistik mit dem Vorbild der Germanistik in Deutschland

2. Interkulturelle Germanistik 
3. Germanistik mit interdisziplinären Fachrichtungen (Richtungen wie auswärtiger Politik sowie Wirtschaft)

4. Germanistik in Verbindung mit natur- oder ingenieurwissenschaftlichen Fachrichtungen

Didaktik und Methodik des Fremdsprachenunterrichts ist offensichtlich kein Lernziel des Germanistik-Studiums. Auch im Beitrag von Wei und Qin (2017) aus der jüngsten Zeit über die Entwicklung von Germanistik in China findet man keine Hinweise über Interesse für die Deutschlehrerausbildung. Die Ziele des Bachelor-Studiums von Germanistik liegen eher auf der Vermittlung von sprachlichem und landeskundlichem Wissen (vgl. Gu 2004, S. 90), wie der Lernplan vom Germanistikstudium an der Universität Nanjing (vgl. Kong 2007, S. 129) zeigt.

Wei (2009, S. 283-285) deutet an, dass die Zielsetzung des Germanistikstudiums in den 50er und 60er Jahren eher für die Ausbildung der sprachlichen Fertigkeiten sowie Literatur- und Sprachwissenschaft gedacht war. Heute haben verschiedene Universitäten und Hochschulen je nach eigener Vorstellung und Rahmenbedingungen verschiedene Zielsetzungen. Li und Lian (2017, S. 125f.) vertreten eine ähnliche Position. In der Zusammenarbeit mit Universitäten in deutschsprachigen Ländern werden auch neue Studienrichtungen entwickelt. Das ist aber stark regional- und universitätsabhängig. Sie bestätigen eben, dass Fremdsprachendidaktik bis heute nur eine Randerscheinung im Germanistikstudium in China ist. Auch interkulturelle Kommunikation und angewandte Linguistik gewinnen erst in der letzten Zeit vergleichsweise mehr an Bedeutung.

Während didaktische Kenntnisse im Studium nicht vermittelt werden, möchten viele Bachelor-Absolvierende Deutschlehrende werden (vgl. Zhu 2019, S. 9). Fehlendes didaktisch-methodisches Wissen führt zu Problemen in der Unterrichtspraxis. Die fehlende Lehrerausbildung stellt offensichtlich ein großes Problem dar.

Für das Masterstudium von Germanistik gibt es nicht viele Informationen. Eine Statistik aus dem Jahr 2008 zeigt, dass die Universitäten nur Masterstudiengänge in Richtung Literatur-, Kultur- und Sprachwissenschaft anbieten (vgl. Wei 2009, S. 287). Laut einer Statistik zur Fachrichtungen der Deutschlehrenden an Germanistikabteilungen kann man auch feststellen, dass die Mehrheit der Lehrkräfte Literatur- und SprachwissenschaftlerInnen sind (vgl. Zhu 2019, S. 11; Wei 2009, S. 296). Wenn die Lehrenden keine methodische Ausbildung gemacht haben, beruhen ihre Unterrichtsmethoden auf eigener Lernerfahrung (vgl. Steinmüller 2019, S. 232; Goldberger 2010, 6; Hunold 2009, S. 63; Li 2009, S. 302). Das heißt, wenn die Person kein Auslandsstudium hat, übt ihre 
Unterrichtserfahrung vom Fremdsprachenunterricht in China großen Einfluss auf ihre eigene Lehrmethode aus, die in den meisten Fällen von Lehrerzentriertheit tief geprägt wird. Raupach (2016, S. 61) demonstriert, dass die chinesischen Masterstudierenden vom Fach-DaF in Deutschland in ihrem Unterrichtspraktikum die Tendenz zeigen, ihre lehrerzentrierte, vom Frontalunterricht geprägte Lernerfahrung übertragen zu wollen.

Die fehlende Lehraus- und Fortbildung hat offensichtlich negative Einflüsse auf die Qualität des Unterrichts (vgl. Hunold 2009, S. 62f.; Gu 2004, S. 93; Thelen 2003, S. 242f.; Becker-Sliwa 2017, S. 47). Das wird auch längst erkannt. Seit Ende der 80er Jahre des letzten Jahrhunderts organisierten DAAD und GoetheInstitut Lehrfortbildungsangebote für chinesische Deutschlehrende in China. Gerbig (2007, S. 319) kommentiert, dass diese Reihen von Regionalseminaren im Großen und Ganzen erfolgreich laufen, aber keinen „Multiplikatoreneffekt “ haben, da die Teilnehmer das Wissen aus dem Fortbildungskurs entweder nicht an der Heimatuniversität weitergeben, oder auf Widerstände stoßen, weil manche Lehrende an der alten traditionellen Methode festhalten wollen. Gu (2004, S. 94) bestätigt die Existenz dieses Phänomens und meint, dass es einen kleinen Teil von Lehrenden gibt, die von der modernen Methode nicht überzeugt sind. Steinmüller (2019, S. 235) meint ebenfalls, dass die Fortbildungsprogramme von Mittlerorganisationen die mangelhafte Qualifikation von Deutschlehrenden nicht verbessern können, weil viele DaF-Lehrenden die vermittelte Methode im Unterricht kaum einsetzen, wie die Forschung von Tian (2017) bestätigt. Auch als Probleme der Fortbildungsprogramme sieht Gu (2004, S. 95), dass die verbesserten Methodenvorschläge in überarbeiteten Curricula von den meisten Lehrenden nicht in der Praxis eingesetzt werden. Steinmüller (2019, S. 234) stützt sich darauf und weist hin, dass, obwohl inzwischen eine beachtliche Gruppe von ausgebildeten jungen chinesischen DaF-Lehrenden von ihrem DaF-Studium in Deutschland zurück nach China kommt, es vielen schwer gelingt, sich gegen die Hierarchie der Abteilung durchzusetzen.

Es kristallisiert sich heraus, dass fehlendes didaktisches Wissen eines der größten Probleme des chinesischen Deutschunterrichts ist. Dabei spielen noch sozio-kulturelle Faktoren eine Rolle. Thele (2003) untersuchte die Fremdsprachen-Methodenentwicklung in China und findet, dass der Einsatz der kommunikativen Methode eine Herausforderung ist:

Viele Fachartikel machen deutlich, dass „kommunikative Kompetenz" ein schwieriger, aufgeladener Terminus ist, hinter dem sich verschiedene Vorstellungen der Benutzer verbergen, beeinflusst durch deren Lehr- und Lerntraditionen... Problematisch erwies sich hierbei schon, dass[...] die fehlende Lehrer-Innenausbildung effektiver Unterrichtsgestaltung häufig im Weg stand und schon daher, was letztendlich als „kommunikative Methode“ in China 
bezeichnet wird, stimmt nicht mit der westlichen Begriffsbestimmung überein. Da die gesellschaftspolitischen Strömungen, die diesen Ansatz im Westen hervor gebracht haben, so weinig mit denen in China vergleichbar sind, diese Unterschiede aber nicht reflektiert werden, erschwert die Adaption des Ansatzes in China zusätzlich (ebenda, S. 240).

Das traditionelle Lehrer-Vorbild-Konzept, das tiefe kulturelle Prägung trägt, übt Einfluss auf die Unterrichtsmethode aus. Dieser Aspekt wird von deutschen und chinesischen Forschenden aus verschiedenen Standpunkten debattiert. Während nach Meinung vieler chinesischer Autoren die kulturelle Lern- und Lehrtradition für der Unterrichtsbesonderheit Rechnung tragen kann (vgl. Zhao 2019, S. 22; Chou 2015, S. 163-169), finden einige deutsche Wissenschaftler, dass der kulturelle Einfluss sehr begrenzt ist (vgl. Mitschian 1999; Steinmüller 2019).

Steinmüller (2019, S. 229-231) widerspricht der „andauernden Wirkung konfuzianischer Unterrichtsprinzipien“, weil Konfuzius' Lern- und Erziehungsmethode für autonomes Lernen durch „eigenständiges entdeckendes Lernen unter Anleitung des Lehrers“ spricht. Das stimmt wohl, dass Konfuzius autonomes Lernen und lernerzentriertes Lehren befürwortet. Allerdings beschreibt die pädagogische Tugendlehre vom Konfuzius nur das ideale Lernen und Lehren, und ist nicht die Grundlage der Autoritätsstellung von Lehrenden in China. Die Autoritätsstellung von Lehrenden kommt von Konfuzius Lebens- und Sozialphilosophie, welche das Leben und Verhalten von Chinesen stärker prägt (vgl. Chou 2015, S. 161). Chou (2015, S. 159f.) stellt die Lebens- und Sozialphilosophie vor und macht folgende Zusammenfassung:

...(Der) Lebens- und Politikphilosophie des Konfuzius[...] sind fünf zwischenmenschliche Grundbeziehungen von zentraler Bedeutung, nämlich die Beziehungen (1) zwischen Herrscher und Untertan, (2) zwischen Vater und Sohn, (3) zwischen älterem und jüngerem Bruder, (4) zwischen Ehemann und Ehefrau sowie (5) zwischen Freund und Freund. In jeder dieser hierarchischen Beziehungen gibt es bestimmte Rechte und Pflichten, außerdem gibt es jeweils ein die Beziehung bestimmendes Prinzip: die Untertanen sollen dem Herrscher mit Treue oder Loyalität gegenübertreten, die Kinder den Eltern mit Pietät und Gehorsamkeit, die Geschwister sollen sich gegenseitig brüderlich behandeln, die Ehefrau soll dem Ehemann mit Gehorsamkeit begegnen sowie der Jüngere dem Älteren mit Respekt (ebenda, S. 160).

Das veranschaulicht die Lehrer-Schüler-Beziehung in China. Die Lehrperson gehört zu der älteren Generation, die respektiert werden soll (vgl. ebenda, S. 168). Auch die Arbeit von deutschen Forschenden bestätigen dieses Phänomen. Hunold (2009, S. 54) erörtert, dass es in asiatischen Ländern üblich ist, die Lehrperson als Vorbild für Lernen und Verhaltensstandards zu betrachten. Diese hierarchische Ungleichheit führt zur einer Lehrer- und Schüler-Beziehung, die man im Westen heutzutage nicht mehr sehen kann. 
Das perfekte moralische und intellektuelle Bild der Lehrperson übt auch Einfluss auf das Unterrichtsverhalten der Lehrenden aus. Hunold (2009, S. 55) findet, dass chinesische Lehrenden ihr ideales Bild vor Schüler perfektionieren müssen, um das Gesicht zu wahren. Im Unterricht versucht die Lehrperson ihre Unvollkommenheit zu verbergen, besonderes bei aussprachlichen Problemen. Das kann dazu führen, dass die Lehrenden absichtlich kommunikationsorientierte Unterrichtsgestaltung vermeiden.

Zusammenfassend lässt sich sagen, dass viele Faktoren beim Einsatz der kommunikativen Methode im chinesischen Fremdsprachenunterricht eine Rolle spielen können. Es gibt in China bis jetzt noch keine eigenständig entwickelte Fremdsprachenmethode im DaF-Bereich. Fehlende methodische und pädagogische Ausbildung übt dabei offensichtlich einen starken Einfluss aus. Auch die sprachliche Ausbildung von Lehrenden kann mangelhaft sein (vgl. Hess 1992, S. 232; Wang 2007, S. 105). Das Ausbildungsdefizit kann zur Verständnisproblemen über den Begriff sowie die kommunikative Kompetenz führen (vgl. Wang 2007, S. 104). Man betrachtet den kommunikativen Ansatz wie einen Widerspruch zum Erlernen des sprachlichen Fundaments.

Außerdem sind viele der Lehrenden Literatur- und SprachwissenschaftlerInnen. Sie lehren Deutsch, aber ihr Forschungsinteresse ist nicht die Unterrichtspraxis (vgl. Wei 2009, 296, 301). Wang (2007, S. 107) deutet an, dass das Forschungsdefizit von der Unterrichtspraxis die Methodenentwicklung verhindern kann. Unterrichtsforschung ist eine eigenständige Wissenschaft, die erlernt werden muss. Fehlendes empirisches Forschungswissen führt zum Missverständnis der Unterrichtspraxis. Es gibt meines Erachtens keine systematische empirische Forschung über die Effizienz der kommunikativen Unterrichtsmethode im Vergleich zum traditionellen Unterricht im DaF-Unterricht in China. Die Frage, ob kommunikativer Methodeneinsatz die Ausbildung von sprachlichem Fundament wie Grammatik und Wortschatz nicht begünstigen kann, bleibt offen.

Schließlich ist die Rolle des kulturellen Einflusses nicht zu vernachlässigen, da sie die Lehrer-Rolle im Unterricht definiert und die Lehrer-Schüler-Beziehung beeinflusst. Das kulturell spezifische Verhaltensmuster hat auf den Lernenden einen ebenso großen Einfluss wie auf den Lehrenden. Darüber wird im nächsten Unterkapitel weiter diskutiert. 


\subsubsection{Lernerbezogene Hindernisse}

Außer Fleiß, Zielstrebigkeit und Leistungsorientierung werden chinesische Studierende in der Fachliteratur zur Didaktik des DaF-Unterrichts oft auch mit ein paar negativen Wörtern beschrieben: schweigsam, passiv, zurückhaltend.

Die Passivität der chinesischen Lernenden wird von den DAAD-Lektoren seit Mitte bis Ende der 80er Jahre unter die Lupe genommen. Die Lektorenberichte beklagen die Passivität der chinesischen Lernenden und stellten fest, dass ihr typisch chinesisches Lernverhalten eine Barriere für interaktiven/kommunikativen Unterricht ist (vgl. Mitschian 1991, S. 34-38; Zeilinger 2006, S. 6f.; Steinmüller 2019, S. 229).

Nach 30 Jahren hat sich die Situation nicht verbessert. Wang (2007, S. 122125) deutet an, dass die im Kapitel 2.2.1 genannten Probleme lehrerseits zu mangelhafter Kommunikationsfähigkeit der Lernenden führen können. Allerdings kann man ähnliche Probleme bei chinesischen Lernenden, die in Deutschland studieren, auch beobachten (vgl. Hunold 2009, S. 52). Wenn es Zurückhaltung und passives Verhalten im Unterricht in beiden Kulturräumen zu beobachten gibt, muss man eine Frage stellen: Was sind die Probleme lernerseits?

Wang (2007, S. 123) und Chou (2015, S. 134) deuten an, dass das komplizierte deutsche Sprachsystem eine wichtige Rolle dabei spielen soll. Der große Unterschied zwischen Chinesisch und Deutsch wird von vielen Didaktikern als Hürde für chinesische Lernende dargestellt. Die Flexion, Genusmarkierung und Wortstellung der deutschen Sprache sind besonders schwierig für chinesische Lernende (vgl. Han 2006, S. 407; Wang 2007, S. 123). In China wird ein Einführungskurs für Phonetik der deutschen Sprache als Notwendigkeit vorgeschlagen(vgl. Gu et al. 2003, S. 92; Zhu 2003, S. 39), weil ein großer Unterschied zwischen dem chinesischen und deutschen phonematischen Systeme besteht (vgl. Wang 1988, S. 77-80; Zhu 2009, S. 650).

Darüber hinaus ist das Sprechen ein komplexes Thema: die Lernenden müssen in allen Aspekten (Aussprache, Grammatik, Wortschatz...) in der Lage sein, sich zu äußern (vgl. Han 2006, S. 407). Huneke und Steinig (2013, S. 161-167) heben hervor, dass Sprechen in der Muttersprache schon ein hochkomplexer psycholinguistischer Vorgang ist, der Aufmerksamkeit und Mut erfordert. Das Sprechen in der Fremdsprache benötigt noch viel mehr Aufmerksamkeit und kultur-spezifisches Wissen.

Wang (2007, S. 123) betont, dass sich die chinesischen Lernenden beim Sprechen eher auf die sprachliche Form konzentrieren. Außerdem tendieren sowohl die Lernenden als auch die Lehrenden, pragmatische Angemessenheit bei der Kommunikation zu vernachlässigen. Die Unsicherheit beim Sprechen wird vom 
komplizierten deutschen Sprachsystem verstärkt, sodass viele chinesische Lernende Angst vor dem Sprechen haben.

Fehlende Kommunikationsmöglichkeiten auf Deutsch spielen dabei auch eine Rolle. Nicht nur diejenige in China haben kaum Kontakt mit Muttersprachlern, auch viele in Deutschland haben wegen Integrationsschwierigkeiten keine Gelegenheit zur Kommunikation mit Deutschen (vgl. Wang 2007, S. 124; Han 2006, S. 409; Hunold 2009, S. 51).

Hinter der Sprechangst verbergen sich auch kulturelle Einflüsse. Dabei spielt die Gesichtswahrung eine bedeutende Rolle. Maletzke (1996, S. 106) weist darauf hin, dass Gesichtsverlust in chinesischer Kultur „den Kern der Persönlichkeit berührt oder gar verletzt bis hin zur Unerträglichkeit“. Han (2006, S. 412) weist darauf hin, dass Chinesen „sehr auf die Reaktion von anderen achten“. Fehler vor den anderen zu machen oder vor der Gruppe von Lehrenden kritisiert zu werden ist unerträglich (vgl. Chou 2015, S. 169). Da der Unterricht in China manchmal mit sehr großen Gruppen stattfindet (siehe Kapitel 2.2.3), ist die Angst vor Gesichtsverlust noch größer.

Mitschian (1999, S. 45-47) vergleicht die Berichte zum Lernverhalten der asiatischen Lernenden in den 80er Jahren und fasst drei kulturelle Ursachenzuweisungen von der Passivität der genannten Gruppe zusammen: Angst vor Gesichtsverlust, Unterordnung unter die Gemeinschaft und die Achtung vor der Autorität. Xie (2010, S. 11f.) stellt auch Forschungsergebnisse im English as a Second Language (EFL)-Raum vor und zeigt, dass das Unterrichtsverhalten von kulturellen traditionellen Werten beeinflusst werden kann. Die sind: Gesichtswahrung, Bescheidenheit, Respekt vor der Autorität und die Kollektivorientiertheit $^{3}$. Auch die Forschung von Hess (2007) teilt die gleiche Auffassung.

In China herrscht offensichtlich eine andere Lerntradition als in Deutschland. Die lehrerzentrierte Unterrichtsform führt dazu, dass die Studierenden an nonverbale Interaktion gewöhnt sind (vgl. Xie 2010, S. 12). Man legt Wert auf Prüfungsleistung und Auswendiglernen von Wissen. Die Wurzel dafür kann in der kaiserlichen Beamtenprüfung und der Bildungsideologie des antiken China gesehen werden.

Chou (2015, S. 166f.) ist der Meinung, dass die kaiserliche Beamtenprüfung auf das moderne Prüfungssystem im chinesischsprachigen Raum immer noch Einfluss ausübt. In China sind die Prüfungen ab der Grundschulzeit bis

3 „They put collective benefits before individual interests and so avoided bothering teachers with questions which might only have concerned themselves. They only raised questions when they could not find out answers unaided.“(Xie 2010, S. 11) 
zur Universität mehrheitlich wissensabfragende Tests. Auswendiglernen ist eine wichtige Lernstrategie, die man beherrschen muss, um gute Leistungen zu bekommen. Chou (2015, S. 168) weist darauf hin, dass die gesamte Gesellschaft des Kulturraums prüfungsorientiert ist, weil Schulleistung auf das Leben eines Individuums starken Einfluss ausüben kann.

Die schulische Sozialisation prägt die Wertstellung von Lernenden für Unterricht. Raupachs Erfahrung mit chinesischen DSH-Lernenden bestätigt, dass sie „formbezogenes Lernen vorziehen“ und „einen expliziten Grammatikunterricht erwarten“ (vgl. Raupach 2016, S. 58). Diese Wertstellung wird auch von Hunold (2009, S. 56) gesehen und darauf hingewiesen: das Bestehen einer Prüfung ist die höchste Lernmotivation von chinesischen Studierenden, und die chinesischen Studierenden sind „pragmatischer orientiert“ als die westlichen. Auch der empirische Befund von Chou (2015, S. 243f.) aus jüngster Zeit bestätigt die Prüfungsund Leistungsorientiertheit von chinesischen Lernenden.

Außerdem kann Auswendiglernen nach asiatischer Tradition zur einer „Vertiefung und Entwicklung des Verstehens“ führen, was mit „mechanischem Lernen" nach westlichem Konzept nicht vergleichbar ist (vgl. Hunold 2009, S. 59). Zahlreiche Studien zeigen, dass ostasiatische Schüler und Studierende „tiefenorientierte Lernstrategien einsetzen“ und „Lernzugänge mit ausgeprägter Verarbeitungstiefe bevorzugen“ (vgl. Hesse 2007, S. 247f.). Helmke (2017, S. 203) merkt eben an, dass „repetitives Üben“ nur im westlichen Denken einen „negativen Beigeschmack“ besitzt:

In Ostasien, ins besondere in den durch das konfuzianische Erbe gekennzeichneten Ländern (CHC = Confucian Heritage Countries) wie China, Japan, Korea und Vietnam hat das wiederholende Üben einen vollkommen anderen Stellenwert. Man spricht gelegentlich auch vom Paradox des chinesischen Lerners (großer Lernerfolg trotz Vorherrschens von Wiederholungsstrategien beim individuellen Lernen) ...

Einige deutsche Forschenden sind der Meinung, dass das passive Lernverhalten von chinesischen Lernenden eher auf schulische Sozialisation als auf kulturelle Prägung zurückgeführt werden kann (vgl. Hunold 2009, S. 60; Mitschian 1999). Meines Erachtens kann man schulische Sozialisation von kultureller Prägung nicht streng abtrennen. So Maletzke (1996, S. 16):

In der Kulturanthropologie ist Kultur im Wesentlichen zu verstehen als ein System von Konzepten, Überzeugungen, Einstellungen, Wertorientierungen, die sowohl im Verhalten und Handeln der Menschen als auch in ihren geistigen und materiellen Produkten sichtbar werden. Ganz vereinfacht kann man sagen: Kultur ist die Art und Weise, wie die Menschen leben und was sie aus sich selbst und ihrer Welt machen. 
Die kulturelle Prägung übt seinen Einfluss in allen Phasen aus, zu jeder Zeit im Leben eines Individuums. Auch die Bildungsinstitutionen werden von der Kultur beeinflusst. Das passive und schweigsame Unterrichtsverhalten von chinesischen Lernenden taucht nicht nur im Fremdsprachenunterricht auf, sondern auch in ihrem Leben und Studium in Deutschland und in China im Allgemeinen (vgl. Hunold 2009, S. 52-54). Mangelhafte Lehrmethodik und lehrerzentrierte schulische Sozialisation können ihr Lernverhalten stark beeinflussen, aber es gibt auch andere Ursachen.

Wenn man die Probleme von chinesischen Lernenden im Fremdsprachenunterricht genau betrachtet, kann man feststellen, dass das Hauptproblem in ihrer Schweigsamkeit im Unterricht liegt. Die Gründe ihrer Schweigsamkeit sind vielfältig. Die Unterrichtsmethode, die institutionellen Rahmenbedingungen, die Lernerfahrung und persönliche Lernmotivation und Charakter sowie viele andere Dinge können das Lernverhalten eines Individuums beeinflussen. Im Kapitel 2.2.4 wird das Konzept Willingness to Communicate vorgestellt, um die Einflussfaktoren der Schweigsamkeit besser zu verstehen. Die kulturellen und unterrichtsmethodischen Einflussfaktoren werden auch im Rahmen dieses Konzepts weiter diskutiert. Davor sollen die Rahmenbedingungen des Fremdsprachenunterrichts in China näher betrachtet werden, um die Lernbedingung und Lernumgebung zu veranschaulichen.

\subsubsection{Hindernisse in den Rahmenbedingungen}

Fehlende Lehrerausbildung, problematische schulische Sozialisation und ein besonderer kultureller Hintergrund sprechen dafür, dass der Einsatz von interaktiver/kommunikativer Methode im chinesischen DaF-Unterricht besondere Schwierigkeiten hat. Die Rahmenbedingungen des Fremdsprachenunterrichts stellen eben eine Hürde dar, weil sie auch von kultureller und historischer institutioneller Entwicklung geprägt werden.

Um die unbefriedigende Qualität des Fremdsprachenunterrichts zu verbessern, wird eine Reihe von Maßnahmen eingesetzt: Diskussionen über Zielsetzung des Fremdsprachenunterrichts, Unterrichtsmuster, Lernkompetenz und -strategien sowie Leistungsmessungsverfahren werden durchgeführt. Darunter spielt das nationale Leistungsmessungsverfahren für Fremdsprachenlernen eine wichtige Rolle. In den nationalen Prüfungen (College Englisch/Deutsch Stufe 4 und 6) werden hauptsächlich grammatisches Wissen und Lesen evaluiert, sodass man sich im Unterricht auf linguistisches Wissen konzentrieren muss (vgl. Wang 2007, S. 96-101). Leistungsorientierte Lerngewohnheiten (siehe Kapitel 2.2.2) können dazu führen, dass sprachliches fundamentales Wissen 
wichtiger als kommunikative Kompetenz des Lernenden betrachtet wird. Auch für die Zulassung zum Master-Studium an chinesischen Universitäten werden wissensabfragende Tests eingesetzt. Die Studierenden, die sich für eine Weiterbildungsmöglichkeit in China interessieren, legen mehr Werte auf Grammatik und Wortschatz.

Außerdem sehen die institutionellen Rahmenbedingungen in China auch anders als die in Deutschland aus. Thele setzte sich mit der Fremdsprachenunterrichtsituation in den 80er und 90er Jahren auseinander, und spricht vom negativen Einfluss der "schlechten Ausstattung“ der Universitäten auf eine Verbesserung von kommunikativer Kompetenz (vgl. Thelen 2003, S. 240). Damals kann man die Universitätsausstattung ja mit dem Wort „schlecht“ bezeichnen. Heutzutage sind die Mehrheiten der chinesischen Universitäten gut oder sogar sehr gut ausgestattet. Die Frage ist aber, ob die gute Ausstattung dem Unterricht wirklich was bringen kann.

Die Sprachlabors, die mit der Einführung von audio-lingualer Methode importiert werden, sind heutzutage an vielen Universitäten zu sehen. An der Technischen Universität Kunming ${ }^{4}$ beispielsweise hat das Sprachlabor einen festen Platz im studienbegleitenden Fremdsprachenunterricht. Die Klassenzimmer des Lehrgebäudes der Fakultät für Fremdsprachen- und Kulturen bestehen nur aus Sprachlabors. Das bedeutet, dass jedes Klassenzimmer ein Raum mit abgetrennten Kabinen ist und keine Tafel besitzt. Da bis zum Jahr 2017 die Hälfte der studienbegleitenden Fremdsprachenkurse „Sprechen und Hören“ üben sollen, müssen solche Stunden nur im Sprachlabor durchgeführt werden. Solche Raumbedingungen sind für kommunikativen Unterricht nicht ideal (Steinmüller 2019, S. 234). Nach Beobachtung der Forscherin wird in vielen Sprachlabor-Stunden nicht gesprochen, obwohl der Kurs für "Sprechen und Hören“ dient. Der Unterrichtsverlauf ähnelt der unteren Beschreibung von Steinmüller (2019, S. 234):

In einem Unterrichtsraum mit Gruppentisch, PC für jeden Studierenden und allen modernen Medien sitzt der Dozent auf einem erhöhten Lehrerpult, schreibt wortlos aus dem geöffneten Lehrbuch einen Text in seinem PC, wirft ihn per Beamer an die Wand, und die Studierenden schreiben diesen Text ebenso wortlos von der Wand ab in ihren eigenen PC - DaF-Unterricht mit modernen Medien.

Auch wenn der Unterricht nicht im Sprachlabor stattfindet, gibt es an vielen Universitäten nur einen Hörsaal mit fixierten Stühlen und Tischen. Schön ausgestattet, aber immer noch unpraktisch für eine Interaktion ${ }^{5}$. Die Klassenzimmer sind

4 Chinesisch:昆明理工大学, Englisch: Kunming University of Science and Technology

5 Freistellbare Stühlen und Tische im kleinen Raum kann man an manchen Fremdsprachenhochschulen finden. 
für frontalen und lehrerzentrierten Unterricht eingerichtet. Die traditionelle Klassenzimmereinrichtung stellt den Lehrenden wenig Freiraum für Interaktion mit den Lernenden zur Verfügung und kann negativen Einfluss auf LehrerSchüler-Interaktionshäufigkeit ausüben (vgl. Stadler-Altmann 2015, 57,59). Die empirische Forschung von Chou (2015, S. 213f.) in Taiwan bestätigt ebenso, dass infrastrukturelle Bedingungen Interaktion, Kommunikation und autonomes Lernen behindern können.

Demzufolge ist die Ausstattung der Universitäten trotz der Modernisierung ungeeignet für den Einsatz der kommunikativen Methode. Die ungünstige räumliche Bedingung kann allerdings durch innovative Raumnutzung der Lehrperson verbessert werden (vgl. Stadler-Altmann 2015, S. 59).

Prüfungsorientiertheit und räumliche Rahmenbedingungen bilden deutlich eine Hürde für lernerzentrierte interaktive/kommunikative Unterrichtsgestaltung. Eine der größten Schwierigkeit für Fremdsprachenunterricht in China stellt allerdings die Größe der Gruppe da. An den meisten Universitäten finden Lehrveranstaltungen wegen der Größe der Unterrichtsgruppe und Lerntradition entweder im Hörsaal oder im Sprachlabor statt. Die Gruppenstärke ist nicht ideal für kommunikativen Unterricht. Weil es manchmal nur 1 bis 2 Deutschlehrende an der Universität gibt, arbeiten manche Lehrende in Gruppen von 70 bis zu 120 Studierenden (vgl. Steinmüller 2019, S. 232; Zhu 2007a, S. 147; Hunold 2009, S. 60). Im Folgenden wird der Großgruppenunterricht unter die Lupe genommen, um diese besondere Schwierigkeit und deren Lösung näher zu betrachten.

\subsubsection{Das Problemfeld „Großgruppenunterricht“}

Die Großgruppe kann auf unterschiedliche Weise definiert werden (vgl. Hess 2007, S. 2). Es wird erörtert, dass in einer Klasse nicht allein die Anzahl der Lernenden die Größe der Gruppen bestimmt. Bei der "gefühlten Gruppengröße“ können Faktoren wie Fachgebiet, Lernziele, Methoden, Ressourcen sowie Lerner- und Lehrereigenschaften eine wichtige Rolle spielen (Yang und Loo 2007, S. 74; Hess 2007, S. 2; Loo 2016, S. 362). Das heißt, dass „die Größe“ der Gruppe von Einflussfaktoren wie Raumbedingungen, methodische Kenntnisse und Erfahrungen der Lehrenden sowie Vorwissen und Motivation der Lernenden bestimmt werden können.

In ihrem Werk Teaching Large Multilevel Classes definiert Hess (2007, S. 2) eine Klasse mit mehr als 30 Lernenden als große Klasse. Loo (2007, S. 21) unterteilt Großgruppen in drei Typen: kleine Großgruppe mit 25 bis 35 Lernenden, mittelgroße Großgruppen mit ca. 50 Lernenden und große Großgruppe mit 60 bis 80 Lernenden. Die vorliegende Forschung bezieht sich hauptsächlich auf 
kleine Großgruppen, allerdings können die zu diskutierenden Faktoren auch für mittlere und große Großgruppen gelten.

Winkler (2011) gibt einen Überblick über die Problemfelder des Großgruppen-Unterrichts folgendermaßen:

Tabelle 2: Die Problemfelder des Großgruppenunterrichts (Winkler 2011, S. 3)

\begin{tabular}{|c|c|}
\hline Allgemeine Problemfelder & Aus der Lehrerperspektive \\
\hline $\begin{array}{l}\text { - größere Heterogenität } \\
\text { - - unterschiedliche Niveaustufen, Lernstile } \\
\text { - Anonymität } \\
\text { - eine Tendenz zu vermehrt passivem } \\
\text { Lernverhalten } \\
\text { - unterschiedliche Aufmerksamkeitsspannen } \\
\text { und eine größere Wahrscheinlichkeit des } \\
\text { Auftretens von Störungen } \\
\text { - die Lerner haben wenig Chancen zu üben } \\
\text { - die üblichen Methoden reichen nicht } \\
\text { mehr aus } \\
\text { - der Unterricht ist schwer zu organisieren } \\
\text { - positives Lernklima und Kooperation } \\
\text { zwischen Lehrenden und Lernenden sind } \\
\text { erschwert } \\
\text { - räumliche und zeitliche Situation ist } \\
\text { belastend }\end{array}$ & $\begin{array}{l}\text { - ein höherer Lehraufwand } \\
\text { - Überlastung aufgrund der hohen } \\
\text { Lerneranzahl } \\
\text { - der Lehrer kann im Unterricht nicht auf } \\
\text { jeden Lernenden eingehen } \\
\text { - eine Vielzahl von Stimuli wahrnehmen } \\
\text { und verarbeiten } \\
\text { - eine große Anzahl von Menschen } \\
\text { ansprechen, einbeziehen und ihren } \\
\text { Erwartungen gerecht werden } \\
\text {.. }\end{array}$ \\
\hline
\end{tabular}

Eine große Gruppe bringt viele Auswirkungen mit sich. In der Gruppe können sich große Differenzierungen hinsichtlich Leistungsgrad, Lerntempo, Sozialverhalten, Interessen, sowie der Stärken und Schwächen der Lernenden verbergen.

Yang und Loo (2007, S. 82) deuten an, dass die Passivität eines der größten Probleme vom Großgruppenunterricht im chinesischen DaF-Unterricht ist. Die Masse gibt den Lernenden eine Art von Anonymität. Die potentiell große Heterogenität sowie die Anonymität führt zu diesem typischen Problem vom Großgruppenunterricht (vgl. Loo 2017, S. 4f., 2012, S. 9, 2016, S. 362).

Eine individuelle Betreuungsmöglichkeit des einzelnen Lernenden ist begrenzt. Die Lernenden können und wollen nicht im Unterricht aktiv sein (vgl. Loo 2017, S. 4). Die Frustration von einzelnen Lernenden kann sich schnell ausbreiten und auf die ganze Klasse auswirken. Das übt negativen Einfluss auf das gesamte Lernklima aus (vgl. Loo 2016, S. 362). 
Für die Lehrenden bedeutet Fremdsprachenunterricht mit Großgruppe einen großen Aufwand. Organisatorische Prozesse kosten viel Zeit. Übliche Methoden werden ineffizient wegen der Größe der Gruppe. Organisation von Aktivitäten wird erschwert wegen zeitlicher oder räumlicher Begrenzung. Es ist schwierig, ein positives Lernklima für den Unterricht zu schaffen. Die Lehrenden sind auf Grund der hohen Lerneranzahl überlastet (vgl. Loo 2017, S. 4; Yang und Loo 2007, S. 75; Wang und Zhang 2011, S. 3).

Trotz all dieser Probleme des Großgruppenunterrichts zeigen Untersuchungen zum Lerneffekt in großen Klassen keinen eindeutigen Unterschied hinsichtlich Lernleistung zwischen Groß- und Kleingruppenunterricht. Bei konventionellen Tests, die Wissen abfragend sind, findet man kaum einen Einfluss von der Größe der Gruppen. Allerdings kann die Gruppengröße eine nicht zu übersehende Rolle bei den sprachproduktiven Kompetenzen, bei den höheren kognitiven Funktionen und bei der Motivation spielen. Die unbefriedigende Interaktion im Großgruppenunterricht kann Probleme der Entwicklung von fremdsprachlichen Kompetenzen im Sprechen und Schreiben sowie der Aussprache verursachen (vgl. Loo 2012, S. 8, 2017, S. 4). Das heißt, dass die Erhöhung und Verbesserung der Interaktion im Großgruppenunterricht die Qualität des Unterrichts begünstigen können.

Die Hattie-Studie macht auch deutlich, dass die Größe der Gruppen keinen großen Einfluss auf den Lernfortschritt ausübt, weil sich die Lehrmethoden in kleinerer und größerer Gruppe nicht unterscheiden (vgl. Hattie 2010, S. 85). Infolgedessen kann man vermuten, dass man eine Großgruppe mit richtigen Methoden auch gut unterrichten kann.

Viele Forschenden vertreten diese Meinung, dass die Unterrichtsmethoden, die Lehrenden und die Lernenden sowie die Organisation des Unterrichts eine bedeutsamere Rolle als die Gruppenstärke spielen sollen (vgl. Loo 2017, S. 4, 2012, S. 8f.; Wang und Zhang 2011, S. 3). Becker-Sliwa (2017, S. 47) schließt sich dieser Meinung an und schreibt in ihrem Bericht über DaF-Unterricht in China, dass auch Forschungsarbeiten aus China zu dem Schluss kommen, dass die Art und die Qualität des Unterrichts für die Lernfortschritte entscheidender als die Größe der Gruppe ist.

Man ist sich darin einig, dass Großgruppenunterricht gleichzeitig Herausforderungen und Möglichkeiten mit sich bringt (vgl. Wang und Zhang 2011, S. 6). Der Schlüssel ist die effiziente Organisation und geeigneter Methodenansatz. Anschließend werden die Möglichkeiten des Großgruppenunterrichts sowie die wichtigen methodischen Prinzipien zur erfolgreichen Durchführung des Großgruppenunterrichts skizziert, um die Herangehensweise mit dieser Problematik anschaulich zu machen. 


\subsubsection{Exkurs: Möglichkeiten des Großgruppenunterrichts}

Trotz der genannten Probleme und Schwierigkeiten bringen Großgruppen auch Vorteile mit sich. In Ländern wie China bietet eine große Lerngruppe Lernchancen für viele Lernenden an, weil die Lernendenzahlen stets wachsen und Lehrkräfte fehlen (vgl. Loo 2012, S. 13; Becker-Sliwa 2017, S. 47). Die große Anzahl und hohe Heterogenität erzeugt Kreativität und Lebhaftigkeit im Klassenraum, mehr Interaktionsmöglichkeiten mit mehr Menschen. Darüber hinaus können die Lehrenden sich mit dieser Erfahrung weiter- und tiefer entwickeln, weil Großgruppenunterricht sehr herausfordernd ist (vgl. Loo 2012, S. 13; Wang und Zhang 2011, S. 3; Hess 2007, S. 4).

Zusammenfassend lässt sich sagen, dass Großgruppenunterricht eine Gelegenheit sowohl für die Lernenden als auch die Lehrenden sein kann, wenn man methodisch damit vorsichtiger umgeht. Die Probleme vom Großgruppenunterricht können mit den richtigen Lehrmethoden verringert oder sogar in Chancen gewandelt werden.

Für Großgruppenunterricht ist es wichtig, die Lernenden im Großgruppenunterricht zu aktivieren. Loo (2012, S. 59f.) betont, dass es keine allein selig machende Methode im Fremdsprachenunterricht gibt. Sie weist nach, dass Lernende zu aktivieren wichtig, aber schwer ist. Hess $(2007$, S. 6) schreibt auch dazu, dass es sehr schwierig ist, die gelangweilte Mehrheit zu motivieren.

Eine Methode, die zur Lernaktivierung führen kann, ist die abwechslungsreiche Unterrichtsgestaltung. Hess (2007, S. 8) erörtert, dass die Abwechslung in einer Großgruppe von größerer Relevanz als in einer Kleingruppe ist, weil man da verschiedene Lerngewohnheiten berücksichtigen muss. Außerdem können abwechslungsreiche Aktivitäten Interesse wecken und die Konzentration der Lernenden verbessern.

Ein übliches Problem vom Großgruppenunterricht ist, dass dem Großteil der Lernenden Stimulation fehlt. Das führt zu einer Art von passiver Haltung, die sich dann auf die gesamte Klasse ausbreiten kann. Ein abwechslungsreicher Unterricht mit vielen Interaktionsmöglichkeiten kann nicht nur die Passivität ändern, sondern auch Gelegenheit für individuelles Lernen und Interaktion bieten (vgl. Loo 2017, S. 5; Becker-Sliwa 2017, S. 51; Schmidjell 2017, S. 14; Winkler 2011, S. 4; Loo 2012, S. 60). Dafür hat eine Großgruppenklasse ihren einzigartigen Vorteil, weil die Anzahl der Lernenden für die Interaktion miteinander mehr als genug ist. Die große Anzahl der Gruppen bietet zahlreiche Lernmöglichkeiten an. Die hohe Heterogenität der Lernenden ermöglicht, einen interessanten, abwechslungsreichen und lernerzentrierten Unterricht zu gestalten (vgl. Hess 2007, S. 2f.). 
Die Abwechslung im Unterricht kann durch verschiedene Methoden realisiert werden: Einsatz von verschiedenen Medien, ein Mix von verschiedenen Sozialformen und Arbeitsformen im Unterricht, Binnendifferenzierung, Angebot von verschiedenen Materialien usw. (vgl.Loo 2017, S. 5; Becker-Sliwa 2017, S. 51; Schmidjell 2017, S. 14f.; Loo 2012, S. 59).

Unter den genannten Methoden zur Gestaltung eines abwechslungsreichen DaF-Unterrichts ist es für die vorliegende Arbeit von Bedeutung, die Durchführung und Organisation der Sozial- und Arbeitsformen unter die Lupe zu nehmen, da die in der empirischen Forschung eingesetzten Sprachlernspiele in der Form von interaktiver Gruppen- und Partnerarbeit durchgeführt worden sind.

Schmidjell (2017, S. 14) plädiert dafür, dass die Sozialformen in großen Gruppen eine der bedeutendsten Rollen spielen sollen. Es ist allerdings keine leichte Aufgabe, geeignete Lehr- und Lernaktivitäten für große Lerngruppen zu finden. Offene Unterrichtsformen können außer Zeitverlust auch Unruhe in der Klasse verursachen. Man soll sich gut überlegen, wie man eine bestimmte Aktivität organisiert (vgl. Loo 2017, S. 5). Goh (2017, S. 33) schließt sich dieser Meinung an und bekräftigt, dass die methodische Durchführung eine Herausforderung ist. Die Lehrperson soll eine Arbeits- und Sozialform auswählen, die allen Lernenden Gelegenheit zum Ausprobieren neuer sprachlicher Mittel gibt. Sie hält es für richtig, Übungen und Aufgaben häufig in Kleingruppen durchzuführen, um die große Gruppe zu aktivieren.

Hess (2007, S. 10f.) weist darauf hin, dass kooperatives Lernen für den Großgruppenunterricht ein notwendiger Bestandteil sein soll, weil die Lehrperson allein die ganze Klasse nicht gleichzeitig betreuen und sich um jeden einzelnen Lernenden kümmern kann. Deswegen können die Lernenden während des Lernens manchmal die Rolle von Lehrenden übernehmen und voneinander lernen. Durch kooperatives Lernen miteinander sind die Lernenden auch mehr in den Lehr- und Lernprozess einbezogen.

Gruppenarbeit kann als Auflockerung des Frontalunterrichts dienen und bietet gute Chancen, die Unterrichtsgestaltung stärker als üblich an individuellen Bedürfnissen und Interessen des einzelnen Lernenden auszurichten (vgl. Meyer 1994, S. 242). So gelingt es der Lehrperson, das Individuum trotz der Größe der Gruppe sichtbar zu machen. Mit Gruppen- und Partnerarbeit können Lehrende mit den Kleingruppen und jedem einzelnen Lernenden im Gespräch bleiben, so dass die Lernenden sich als Einzelne wahr- und ernstgenommen fühlen. Diese Personalisierung kann positive Wirkung haben (vgl. Goh 2017, S. 36; BeckerSliwa 2017, S. 51).

Loo (2017, S. 6, 2016, S. 363), Hess (2007, S. 112) und Meyer (1994, S. 245) verdeutlichen, dass Partner- und Gruppenarbeit Kommunikationsgelegenheiten 
im Unterricht bieten können. Die Lernenden werden dadurch aktiver. Haß (2017b, S. 307) weist ebenso darauf hin, dass Gruppenarbeit besonders bedeutsam für den Fremdsprachenunterricht ist, weil die individuelle Sprechzeit der Lernenden dadurch deutlich erhöht werden kann.

Diese „Aufsplitterung in Grüppchen“ (Loo 2017, S. 7) kann die Publikumshemmung vermeiden. Die Lernenden haben in kleiner Gruppe mehr Chancen, ihre Sprechfertigkeit zu üben. Das hat einen positiven Effekt für die Großgruppe, weil die Lernenden sich sicherer fühlen. Die Lernenden können sich ohne Scheu äußern. Das ist für chinesische Lernende von besonderer Bedeutung, da der kulturspezifische Gesichtsverlust und das Schamgefühl von der Größe der Gruppe abhängig sein können. Die Beurteilung durch die anderen wird in der chinesischen Beziehungsarbeit mehr beachtet als die Selbstbeurteilung. Das führt in der chinesischen Interaktion dazu, dass man in größeren Gruppe das Gesicht verstärkt wahren will. Je kleiner die Gruppe wird, desto weniger hat man Angst vor Kommunikation und Meinungsäußerung (vgl. Stork und Zhao 2009, S. 42-44).

Schwerdtfeger (2007, S. 254f.) befürwortet ebenfalls, dass Gruppenarbeit Ängste verringern und die Veränderungen im Verhalten von Lernenden verursachen kann. Die stärkeren Lernenden werden zurückhaltender, während die schwachen Lernenden mutiger werden. Gruppenarbeit erleichtert das Sprechen in einer Fremdsprache. Sie erörtert: „Gruppenarbeit und Partnerarbeit haben so einen systematisch begründeten unverzichtbaren Ort im Fremdsprachenunterricht, ebenso wie Formen des Frontalunterrichts." (ebenda, S. 255)

Gruppen- und Partnerarbeiten im Fremdsprachenunterricht können auch dazu beitragen, die Motivation der Lernenden zu verbessern, weil sich die Lernenden in der Gruppe anerkannt fühlen können. Das autonome Lernen wird gefördert, da die Lernenden selbständig mit anderen Lernenden arbeiten. Die Lehrperson tritt in den Hintergrund, wird als Helfende gesehen. Außerdem wird die individuelle Sprechzeit der Lernenden erhöht. Gruppenarbeit kann auch ein Zusammengehörigkeitsgefühl in der Klasse entwickeln und festigen, kann die Selbständigkeit und Solidarität der Lernenden fördern, wenn ein Gruppenunterricht zielstrebig gestaltet wird. (vgl. Schiffler 1985, S. 148-151; Becker-Sliwa 2017, S. 48-51; Meyer 1994, S. 245).

Allerdings hat Gruppen- und Partnerarbeit wie alle anderen Methoden im Fremdsprachenunterricht ihre Nachteile. Meyer (1994, S. 251) deutet an, dass der Gruppenunterricht eine attraktive und nahezu einhellig befürwortete Sozialform ist, aber in der Wirklichkeit schwierig realisiert werden kann. Der größte Nachteil ist der hohe Aufwand. Eine gute Gruppenarbeit benötigt eine intensivere Vor- und Nachbereitung als Frontalunterricht (vgl. Meyer 1994, S. 253; Schiffler 1985, S. 153; Rösler 1994, S. 130). Gruppenarbeit in einer Klasse mit 
vielen Teilnehmenden bedeutet manchmal viele vorzubereitende Materialien, welche für die Lehrperson eine Überlastung sein kann. Der andere Nachteil vom Gruppenunterricht in Großgruppen ist der hohe Zeitfaktor (vgl. Loo 2012, S. 105). Aktivitäten in Großgruppen zu organisieren kann Reibungsverlust verursachen (vgl. Loo 2017, S. 7). Kontrollverlust und Disziplinschwierigkeiten können ebenfalls auftauchen (vgl. Loo 2012, S. 103).

Außerdem macht man sich Sorgen um die erhöhte Nutzung der Muttersprache in der Gruppenarbeit (vgl. ebenda, S. 107). Schiffler (1985, S. 153) betont dagegen, dass die Kommunikation in der Gruppe die Bewältigung der Aufgabe fördert, auch wenn die Lernenden in der Gruppe in der Muttersprache sprechen. Meyer (1994, S. 253) pointiert, dass die Risiken sich lohnen, weil der scheinbar risikoreichere Gruppenunterricht lebendiger, interessanter und letztlich befriedigender als der Frontalunterricht ist.

Der Reibungsverlust kann durch straffe Unterrichtsorganisation verringert werden. Die wichtigste Voraussetzung dafür ist sorgfältige Planung und Vorbereitung des Unterrichts, die von Lernzielen und Zielaufgaben ausgehen (vgl. Loo 2017, S. 7f.; Goh 2017, S. 33). Über die Planung und Durchführung des Gruppenunterrichts in der Praxis beschäftigt sich das Kapitel 6 weiter, am Beispiel von Sprachlernspielen anhand der empirischen Untersuchung.

\subsubsection{Willingness to Communicate}

Die Schweigsamkeit chinesischer Lernender ist nicht nur im Deutschunterricht zu beobachten. Im Englisch als Fremdsprachenunterricht wird dieses Phänomen auch bemerkt (vgl. Xie 2010, S. 10). Wen (2003, S. 18f.) argumentiert, dass es eine weitverbreitete Erkenntnis ist, dass chinesische Englischlernende sehr gute Leistungen bei Grammatik-basierten Prüfungen bringen können, aber sehr schweigsam im Unterricht sind: Ihnen fehlt es an Kommunikationsbereitschaft. Im EFL-Raum wird das Konzept Willingness to Communicate (WTC) in den 90er Jahren entwickelt, um die Schweigsamkeit der Fremdsprachenlernenden im Unterricht zu verstehen und zu beseitigen.

Der Ursprung von WTC führt zur Forschung für Kommunikationswilligkeit in der ersten Sprache zurück. Laut Macintyre und Dörnyer (1998, S. 545f.) wird WTC im Jahr 1985 in der Literatur für Kommunikation in der ersten Sprache als „the probability of engaging in communication when free to choose to do so" vorgestellt und verstanden.

Forschungen bestätigen, dass WTC in der Muttersprache eines Individuums von vielen Faktoren beeinflusst werden kann, wie z. B. selbst-wahrgenommene Kommunikationskompetenz, Kultur, Kommunikationsangst, Schüchternheit 
usw. (vgl. Macintyre et al. 1998, S. 546; Zarrinabadi und Tanbakooei 2016, S. 30f.). Während die WTC der ersten Sprache eher von Charaktereigenschaften geprägt sind, kann WTC der Fremdsprache von sowohl Charaktereigenschaften als auch situationsabhängige Faktoren beeinflusst sein (vgl. Zarrinabadi und Tanbakooei 2016, S. 31). Dazu schreibt Macintyre und Döryner (1998, S. 546):

It is highly unlikely that WTC in the second language (L2) ist a simple manifestation of WTC in the L1... The differences between L1 und L2 WTC may be due to the uncertainty inherent in L2 use that interacts in a more complex manner with those variables that influence $L 1$ WTC...

Basierend auf ihren Forschungen entwickeln Macintyre und Dörnyer (1998) das Pyramidenmodell der Einflussfaktoren von WTC, das von vielen WissenschaftlerInnen in ihren Forschungen zur WTC referiert und eingesetzt wird (vgl. Zarrinabadi 2014, S. 289; Kang 2005, S. 280; Zhang et al. 2018, S. 230; Zarrinabadi und Tanbakooei 2016, S. 33; Pawlak et al. 2015, S. 655; Wen und Clément 2003, S. 23-27).

Ihr Modell zeigt, dass L2 WTC von individuellen Charaktereigenschaften bis zu den verschiedenen Faktoren der Kommunikationssituationen sowie der soziokulturellen Rahmenbedingungen beeinflusst werden kann. L2 WTC wird anhand dieses Modells als „a readiness to enter into discourse at a particular time with a specific person or persons, using a L2“" (Macintyre et al. 1998, S. 547) definiert.

Die Erhöhung von WTC soll als wichtigstes Ziel des Fremdsprachenlernens betrachtet werden. Macintyre und Dörnyer finden, dass die Erhöhung von WTC zu dem Endziel vom Fremdsprachenlernen beitragen kann: die Kulturen und Nationen mit Hilfe von Kommunikation zusammenzubringen (vgl. ebenda, S. 559).

Zahlreiche empirische Forschungen zur WTC werden durchgeführt, weil man herausfinden will, warum manche Lernende in Fremdsprachen kommunizieren wollen und manche nicht. Lernende mit höherer WTC werden aktivere Lernende, die mehr Bereitschaft zur Kommunikation in einer Fremdsprache zeigen und mehr Lernmöglichkeiten bekommen würden (vgl. Zarrinabadi 2014, S. 289). Die Forschungen zur WTC können dabei helfen, kommunikatives Verhalten und die Psychologie der Fremdsprachenlernenden besser zu verstehen und didaktische Verbesserungsmöglichkeiten zur Interaktion im Fremdsprachenunterricht zu gestalten (vgl. Peng und Woodrow 2010, S. 835).

Es gibt viele Forschungen, die die Einflussfaktoren relativ stabiler persönlicher Charaktere untersuchen (vgl. Zhang et al. 2018, S. 229). Es wird allerdings argumentiert, dass der Forschungsschwerpunkt auf die dynamische Natur von 


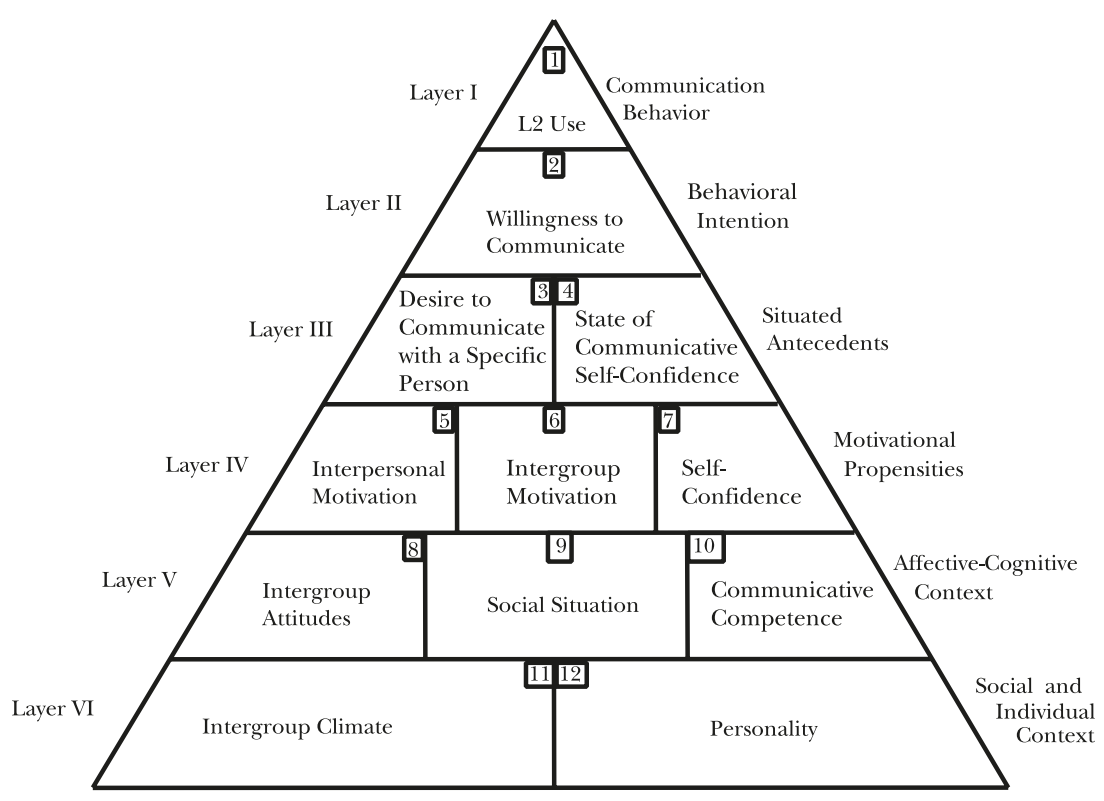

Abbildung 1: Heuristic Model of Variables Influencing WTC (Macintyre et al. 1998, S. 547)

WTC gelegt werden soll, weil die Forschungen auf der Ebene der Charaktereigenschaften widersprüchliche Resultate zeigen (vgl. ebenda, S. 227).

Nach Zarrinabadi (2014, S. 289f.) und Kang (2005, S. 279) zeigen die empirischen Forschungen auf "trait-like“ Ebene, dass die selbst wahrgenommene Kommunikationskompetenz und Kommunikationsangst die WTC am stärksten prägen kann. Auch andere persönliche Variablen können dabei eine Rolle spielen: Motivation, Selbstvertrauen, Geschlecht ${ }^{6}$, Alter ${ }^{7}$ und Einstellungen (vgl. Zarrinabadi 2014, S. 289; Zarrinabadi und Tanbakooei 2016, S. 31; Cao und Philp 2006, S. 481).

Viele der Forschungen auf situativer Ebene basieren auf dem Pyramidenmodell von MacIntyre und Dörynei (1998). Zhang (2018) analysiert 35 empirische

6 „Females shows higher level of WTC compared to Males.“(Zarrinabadi und Tanbakooei 2016, S. 31.)

7 "The persons degree of WTC increases with age“(Zarrinabadi und Tanbakooei 2016, S. 31.) 
Forschungen, die die situativen Komponenten von WTC untersuchten, und findet heraus, dass die Gruppenmitglieder (Mitlernende und Lehrende), die Atmosphäre im Unterricht, Themen und Aktivitäten im Unterricht die WTC von Fremdsprachenlernenden beeinflussen können.

Es lässt sich zeigen, dass beide Ebenen, Eigenschaften eines Individuums und Situation der Umgebung, bei der WTC des Fremdsprachenlernens eine Rolle spielen können. Dazu erörtert Zarrinabadi (2014, S. 290):

The trait-like and situational views of WTC are found to complement each other. Trait-like WTC prepares individuals for communication by creating a tendency for them to place themselves in situations where communication is expected, while situational WTC affects the decision to initiate communication in specific situations.

Zusammenfassend lässt sich sagen, dass die WTC der Fremdsprache eines Individuums von vielen verschiedenen Variablen beeinflusst werden kann. In der vorliegenden Forschung wird nicht versucht, die empirischen Ergebnisse aller WTC- Forschungen darzustellen und zu diskutieren, sondern nur manche Forschungsergebnisse unter die Lupe zu nehmen, die für die chinesischen Lernenden und die Lehrmethodenentwicklung in China eine besondere Relevanz haben. Die Variablen werden auf zwei Ebenen diskutiert: kulturell und didaktisch.

Fremdsprachenunterricht ist ein Ort, wo verschiedene Kulturen in Kontakt gebracht werden. Im Fremdsprachenunterricht finden Interaktionen statt. Jede Kultur hat eigene Interaktionssitten. Kulturelle Einflüsse spielen dabei offensichtlich eine Rolle. Es ist kulturell geprägt, wie, wann, wieviel, wie oft, mit wem eine Person mit anderen Menschen in ihrem Kulturraum kommunizieren kann oder soll. Solche kulturelle Prägung beeinflusst unsere alltägliche Kommunikation in der Muttersprache und auch in Fremdsprachen.

McCroskey (1990, S. 74) deutet an, dass kommunikative Normen von Kulturen stark abhängig sind und die Kommunikationskompetenz eines Individuums kulturgebunden ist. Er ist der Meinung, dass man aus verschiedenen Kulturen unterschiedliche Vorstellungen von „Schweigsamkeit“ oder „Redefreudigkeit“ haben kann. Man hat deswegen auch ein unterschiedliches Verständnis von der Höhe der WTC eines Individuums in verschiedenen Kulturen. Seine Analyse (ebenda) von WTC Studien in Australien, Mikronesien, Porto Rico, Schweden und der USA bestätigt, dass es große Unterschiede zwischen WTC-Werten unter der genannten Kulturen gibt: Die Amerikaner zeigen die höchste WTC und die mikronesischen Menschen die niedrigste.

Wenn man die Forschungen von WTC vom EFL-Untersuchungsraum beobachtet, kann man feststellen, dass die Mehrheit davon in asiatischen Ländern 
durchgeführt wird (vgl. Zhang et al. 2018, S. 229). Die asiatischen Fremdsprachenlernenden zeigen in der Tat auch mehr Probleme bei der Kommunikation und Interaktion im Fremdsprachenunterricht. Es wird bestätigt, dass asiatische Lernende im Unterricht Passivität, Schweigsamkeit und Kooperationsunfähigkeit in der Gruppenarbeit zeigen (vgl. Cao und Philp 2006, S. 482). Es ist durchaus verständlich, dass die Mehrheit der WTC-Forschungen in diesen Ländern durchgeführt wird, weil man versucht, mit Hilfe der Untersuchungen die Unterrichtsqualität vor Ort zu verbessern.

Peng (2010, S. 838) und Wen (2003, S. 18) weisen darauf hin, dass WTC von Fremdsprache von großer Bedeutung für den Fremdsprachenunterricht in China ist, weil chinesische Fremdsprachenlernende oft als passive Lernende mit mangelhafter WTC im Fremdsprachenunterricht bezeichnet werden. Bis jetzt gibt es leider nur begrenzte Forschungen zur WTC von chinesischen Fremdsprachenlernenden. In der vorliegenden Arbeit werden zwei Forschungen davon dargestellt.

Die Forschung von Peng und Woodrow (2010) wurde an einer chinesischen Universität von Ostchina mit 579 Bachelor-Studierenden durchgeführt. Ihre Forschung zeigt, dass Selbstvertrauen der wichtigste Einflussfaktor von WTC der Zielgruppe ist (vgl. ebenda, S. 855-860). Dieses Ergebnis stimmt mit anderen WTC-Forschungen überein, dass die selbst wahrgenommene Kommunikationskompetenz eines Individuums die WTC bestimmen kann. Das Ergebnis zur Motivation zeigt, dass hohe Motivation keine hohe WTC bedingen muss. Das wird in einer Forschung in Japan auch bestätigt. Peng und Woodrow argumentiert (2010, S. 855), dass die Motivation des Fremdsprachenlernens von chinesischen Studierenden, nicht auf Kommunikation, sondern auf Bestehen der Prüfungen liegt. Ihre Motivation beeinflusst deswegen eher das Erlernen von Wortschatz, Leseverständnis und Schreiben, weil diese bei den Prüfungen eine viel größere Rolle als das Sprechen selbst spielen. Das führt noch dazu, dass die Studierenden von kommunikativen Übungen weniger als von Grammatikübungen überzeugt sind und kommunikationsorientierter Unterricht nicht ernsthaft genug angenommen wird (vgl. ebenda, S. 855f.). Außerdem wird dabei herausgefunden, dass hohe Zusammengehörigkeit in der Gruppe zur Erhöhung der WTC beitragen kann. Darüber hinaus ist festzustellen, dass die Unterrichtsumgebung direkten Einfluss auf Motivation, Selbstvertrautheit und Vorstellung der Lernenden üben kann. Das heißt, dass eine gute Atmosphäre im Unterricht und positive Erfahrung mit Interaktion die Kommunikationsmotivation der Lernenden begünstigen können (vgl. ebenda, S. 856-858).

Während die empirische Forschung von Peng und Woodrow (2010) die Einflussfaktoren in der Unterrichtspraxis belegt, versucht Wen und Clément (2003) 
anhand des Pyramidenmodells die kulturellen Einflüsse auf WTC von chinesischen Lernenden zu analysieren. Sie sind der Meinung, dass die fehlende WTC von chinesischen Lernenden nicht auf sprachliche Problemen, sondern auf die traditionelle chinesische Philosophie und Kultur zurückgeführt werden kann. Die Außenorientierung („other-directed self“) und das Wahren des Gesichts sind Merkmale der kollektivistischen Kultur. Chinesen legen deswegen großen Wert auf die Beurteilung durch Andere. Die Zurückhaltung chinesischer Fremdsprachenlernenden wird von ihrer Angst vor der Beurteilung ihrer Kommilitonen und Lehrenden verursacht und führt zur Schweigsamkeit im Unterricht (vgl. ebenda, S. 19f.). Außerdem sind chinesische Lernenden von der Lehr- und Lerntradition stark geprägt, die Autorität des Lehrers und das Auswendiglernen für wichtig erachtet. Sie weisen darauf hin, dass Lehrerrollenwechsel trotz Lehrerfortbildung und anderer lernerorientierter Methoden nur zu Misserfolgen führt. Die Studierenden können die Rollenwechsel von Lehrenden nicht akzeptieren und fühlen, dass sie im lernerzentrierten Unterricht nicht effizient lernen können. Die Lernenden sind daran gewöhnt, Grammatik als richtiges Wissen wahrzunehmen. Sie führen ihre mangelhafte Kommunikationsfähigkeit oft auf fehlendes Grammatikwissen zurück (vgl. Wen und Clément 2003, S. 22f.).

Basierend auf dem Pyramidenmodell (siehe Abbildung 1.) deuten Wen und Clément an, dass die Zusammengehörigkeit innerhalb der Gruppe und die Rolle der Lehrenden auf sozialer Ebene (Layer VI von Pyramidenmodell) die WTC von chinesischen Fremdsprachenlernenden beeinflussen können. Gute Zusammenzugehörigkeit führt zu besserer WTC. Positive Zusammengehörigkeit in der Gruppe bedeutet im chinesischen Wertesystem, dass die Gruppenmitglieder eine enge Beziehung zueinander haben und ein Gefühl der Einheit entwickelt werden kann.. Das Zusammengehörigkeitsgefühl ist aber im Unterricht mit einer großen Gruppe schwer zu verwirklichen, da die Studierenden sich vernachlässigt fühlen können. Gerade in China ist Unterricht in einer Großgruppe sehr üblich, was offensichtlich negative Auswirkungen auf die Zusammengehörigkeit haben kann. Darüber hinaus spielt die Unterstützung von Lehrenden auch eine wichtige Rolle. Verhalten und Vorstellungen der Lehrperson üben signifikante Wirkung auf die Unterrichtpraxis aus. Da die Lehrenden in China als die Autorität im Klassenzimmer gehalten werden, können sie besonders starken Einfluss auf das Verhalten von Lernenden ausüben. Je engagierter die Lehrperson ist, desto besser kann sie die WTC der Lernenden fördern (vgl. ebenda, S. 26-28). Diese Argumentation wird von Hattie (2010, S. 238) hervorgehoben, dass die Lehrperson die entscheidende Rolle im Unterricht spielt.

Bezüglich der Ebene der Charaktereigenschaften im Pyramidenmodell stellt Wen und Clément (2003, S. 29-31) fest, dass die Risikobereitschaft und Toleranz 
für Mehrdeutigkeit in diesem Kontext von besonderer Bedeutung sind. Risikobereitschaft im Fremdsprachenunterricht bedeutet die Bereitschaft, in peinlichen Situationen zu sein oder von den anderen Mitlernenden ausgelacht $\mathrm{zu}$ werden. Die Lernenden mit hoher WTC sind in der Lage, vor Zuschauern unbekannte komplizierte sprachliche Mittel zu nutzen. Chinesische Lernende, die aus kulturellem Grund Sorgen des Gesichtsverlusts vor anderen haben, tendieren dazu, im Fremdsprachenunterricht wenig zu sprechen, um die schlimmste soziale Peinlichkeit zu vermeiden. Chinesen zeigen auch deswegen eine niedrige Toleranz gegenüber Mehrdeutigkeit. Sie fühlen sich wohl, wenn sie durch regelhafte Grammatik jede Kleinigkeit der Sprache erklären, um jeden kleinen Fehler zu vermeiden, sodass sie das Gesicht wahren können. Solche Überlegungen führen zur Mutlosigkeit des Sprechens. Auf der Motivationsebene finden Wen und Clément (2003, S. 31), dass die chinesischen Lernenden motiviert sind, für die Zugehörigkeit der Gruppe bzw. die Anerkennung der Gruppenmitglieder zu arbeiten. Das heißt, dass Unterricht mit Gruppenarbeit eine gute Methode sein kann, um die Lernenden zu motivieren. Gruppenarbeit wird auch von den Forschungen zum Großgruppenunterricht als wichtiges Instrument bezeichnet (siehe Kapitel 2.2.3.2).

Anhand dieser zwei Forschungen zur WTC von chinesischen Fremdsprachenlernenden kann festgestellt werden, dass kulturelle Faktoren eine bedeutende Rolle dabei spielen. Das heißt aber nicht, dass man das passive Unterrichtsverhalten von chinesischen Fremdsprachenlernenden nicht verbessern kann. Manche Verhaltensweisen haben wahrscheinlich ihre Wurzeln in der Kultur, aber passende methodische Vorgehensweisen können nicht nur negative Auswirkungen der kulturellen Einflüssen verbessern, sondern auch die kulturellen Einflüsse nutzen.

Das starke Zugehörigkeitsbedürfnis kann beispielsweise zur besseren Motivation in der Gruppenarbeit führen. Die niedrige Toleranz für Mehrdeutigkeit spricht für den starken Wunsch zum richtigen Sprachengebrauch.

Laut Cao (2006, S. 487f.) fühlt man sich in kleinerer Gesprächsgruppe in Bekanntschaft sicher und die WTC ist höher. Pawlack (2015, S. 666) bestätigt, dass die Bereitschaft zur Interaktion in kleiner Gruppe am höchsten und im Plenum am niedrigsten ist. Um die Risikobereitschaft zu erhöhen, kann man den Großgruppenunterricht in kleine Gruppen aufteilen und die gegenseitige Bekanntschaft der Lernenden fördern, um das unsichere Gefühl vor anderen zu verringern.

Die Studie von Kang (2005) zeigt, dass außer der menschlichen Beziehung innerhalb der Gruppen auch die Relevanz und Bekanntheit der Gesprächsthemen im Fremdsprachenunterricht starke Auswirkung auf die WTC von 
Lernenden haben können. Lernende meinen, dass ihre Zurückhaltung manchmal vom fehlenden Wissen zu Gesprächsthemen verursacht wird. Zarrinabadi (2014) versucht in seiner Forschung, die Einflussfaktoren lehrerseits auf WTC zu untersuchen. Die Forschung zeigt, dass eine lange Wartezeit auf die Antwort von Lernenden, eine passende Fehlerkorrekturmethode, verbale und nonverbale Unterstützung und geeignete Themenauswahl die WTC der Lernenden beeinflussen können.

Es lässt sich sehen, dass richtige didaktische Überlegungen eine Verbesserung von WTC und Lernverhalten im Unterricht erzeugen können. Geeignete Übungstypen und die richtige Durchführungsmethode können die Unterrichtsqualität verbessern. Aubrey (2011, S. 237) schreibt dazu: „By giving careful consideration to influences on WTC, teachers can manipulate classroom conditions and tap into students' latent WTC to optimize student interaction."

\subsection{Zwischenfazit}

In diesem Kapitel werden die Schwierigkeiten vom Einsatz der interaktiven/ kommunikativen Methode im DaF-Unterricht in China ausführlich diskutiert. Die fehlende Lehrausbildung, das kulturell- und sozialspezifische Lern- und Lehrverhalten sowie die Unterrichtsrahmenbedingungen stellen Hürden für die Verbesserung der Interkation im Unterricht und kommunikativer Kompetenz der Lernenden dar.

Die Diskussion kristallisiert allerdings heraus, dass es Möglichkeiten gibt, den Unterricht interaktiver und kommunikativer zu gestalten. Probleme der Räumlichkeit können durch Lehrverhalten verbessert werden (siehe Kapitel 2.2.3). Passive Lernverhalten können mit richtiger Methode bewältigt werden (siehe Kapitel 2.2.4). Auch die Großgruppe kann Chancen für abwechselungsreiche Interaktion und Kommunikation anbieten (siehe Kapitel 2.2.3.2).

Für chinesische Fremdsprachenlernende verspricht eine erhöhte WTC die Verbesserung des Unterrichtsverhaltens. Die Frage ist, wie man ihre WTC fördern kann. Auch Huneke und Steinig (2013, S. 166) sind der Meinung, dass der Sprachunterricht unbedingt eine ermutigende Atmosphäre braucht, um Anlässe und Situationen für Kommunikation zu schaffen.

Basierend auf der oben genannten Diskussion wird das Sprachlernspiel als Unterrichtsmethode in der vorliegenden Forschung ausgewählt, um die Unterrichtsqualität und die Schweigsamkeit sowie Passivität der chinesischen Lernenden zu verändern. Da Sprachlernspiele meistens in Form von Gruppen- oder Partnerarbeit durchgeführt werden, ist diese Form für den Großgruppenunterricht von besonderer Bedeutung. Die Forschung von Pawlak (2015) zur WTC 
deutet daraufhin, dass die Lernenden große Kommunikationswilligkeit während spielerischer Übungen zeigen. Spielerische Übungen können in kleiner Gruppe durchgeführt werden und helfen dem Aufbau von Bekanntschaft der Lernenden. Sie sind risikofrei und fördern Gruppenzugehörigkeit. Auch andere Forschungen zur WTC zeigen, dass Sprachlernspiele für die Erhöhung der WTC der Lernenden geeignet sein, weil die Beziehung in der Gruppe und Unterrichtsatmosphäre die WTC prägen können (siehe Kapitel 2.2.3.2). Im folgenden Kapitel wird die Unterrichtsmethode Sprachlernspiel diskutiert, um die Eigenschaften und Effekte dieser Methode zu veranschaulichen. 



\section{Sprachlernspiele: Begriff und Forschungsstand}

In der heutigen Fremdsprachendidaktik ist bereits akzeptiert, dass Spiele zum Verstehen und Gebrauch einer Fremdsprache beitragen können (vgl. Dauvillier und Meese 2004, S. 20) und als fester Bestandteil des Fremdsprachenunterrichts angesehen werden sollten (vgl. Jentges 2007, S. 21). Die zahlreichen Spielesammlungen und Spielmaterialien, die seit der kommunikativen didaktischen Wende auf dem Markt zu finden sind, sowie die Tatsache, dass sich ein Band des Fernstudienprojekts zur Fort- und Weiterbildung im Bereich Germanistik und Deutsch als Fremdsprache dem Thema "Spiele im Deutschunterricht“ (Dauvillier und Meese 2004) widmet, sprechen dafür (vgl. Mátyás 2009, S. 114).

Weil es keine allgemeine Definition für ein Spiel gibt, gibt es auch in der Fachliteratur bis heute keine einheitliche Definition zu dem Begriff Sprachlernspiel. Für die vorliegende Forschung ist es wichtig, zuerst einen Definitionsversuch des Sprachlernspiels aus meinem Blickwinkel zu liefern (siehe Kapitel 3.1 und 3.2). Danach werden die erwarteten Effekte von Sprachlernspielen dargestellt (siehe Kapitel 3.3). Das Kapitel 3.4 liefert einen Überblick zu den empirischen Forschungen von Sprachlernspielen im europäischen und asiatischen Fremdsprachenunterricht.

\subsection{Zur Definition vom Spiel im allgemeinen Sinn}

Viele Spielautorinnen und -autoren sind sich darin einig, dass es schwierig ist, eine einheitliche Definition für den Begriff Spiel zu finden (vgl. Kacjan 2010, S. 1179; Klippel 1980b, S. 36; Kilp 2003; ebenda, S. 91; Steinhilber 1986, S. 6; Grätz 2001, S. 5).

Klippel (1980) gibt einen Überblick über die drei Möglichkeiten der Begriffsklärung, die in der Literatur zu finden sind. Dies sind „Definitionsversuche“, "phänomenologische Klärung“ und „Kontrastierung mit vermeintlich gegensätzlichen Phänomenen“ (vgl. Klippel 1980b, S. 36) (siehe Abbildung 2).

Die Kontrastierungsmethode zur Begriffsklärung des Spieles wird in der Literatur vielfach benutzt. Am häufigsten wird Arbeit als gegensätzlicher Begriff zum Spiel genannt. Klippel (1980b, S. 45f.) weist nach, dass es drei Positionen zu den Verhältnissen von Spiel und Arbeit gibt. Ältere Spieltheoretiker teilen die Ansicht, dass Spiel und Arbeit ein Gegensatzpaar bilden, während die neueren Theorien behaupten, dass Spiel und Arbeit Komplementärmengen sind. Eine 


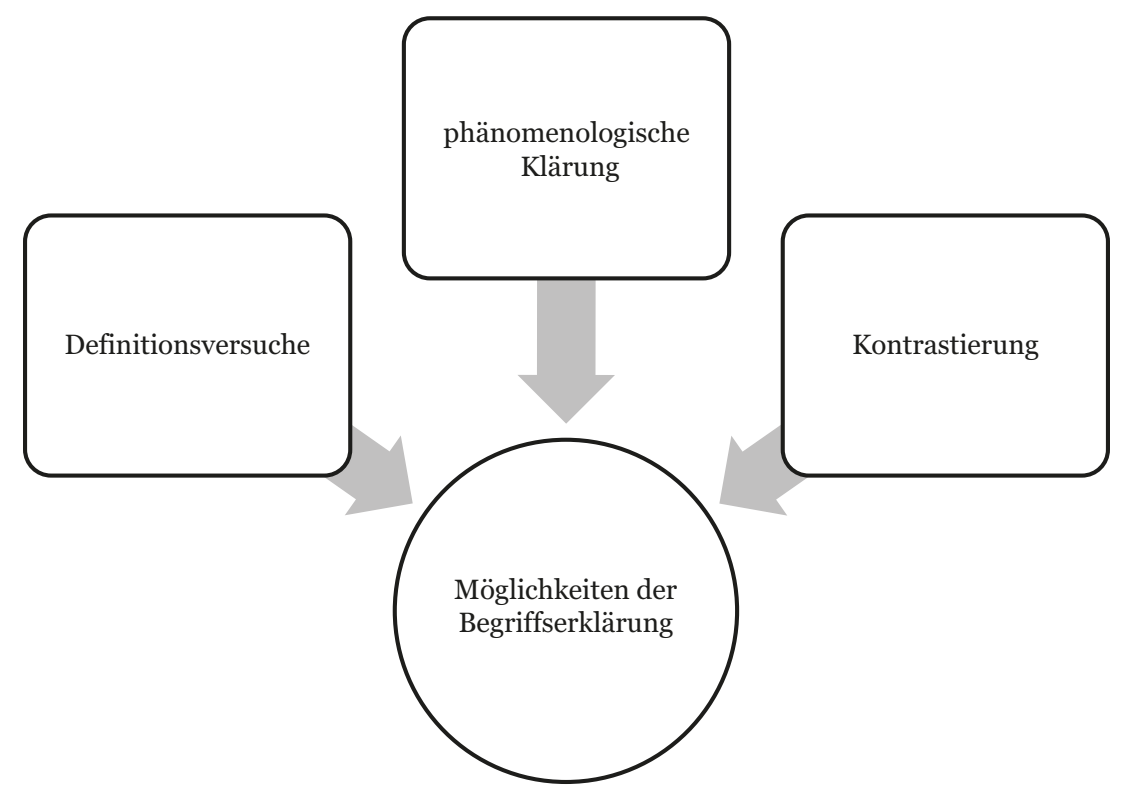

Abbildung 2: Drei Möglichkeiten der Begriffserklärung nach Klippel (1980b)

dritte Stellungnahme plädiert dafür, dass das Spiel als Vorbereitung eines Kindes auf die Tätigkeit eines Erwachsenen angesehen werden soll.

Es wird selten versucht, den Spielbegriff mit umfassender und ausführlicher Definition zu erklären (vgl. ebenda, S. 36). So Steinhilber(1986, S. 6):

Die Fülle der Erscheinungsformen und die komplizierte Struktur des Spiels haben auch zu der Auffassung geführt, eine zufriedenstellende Begriffsbestimmung des Spiels könne nicht geleistet werden.

Etymologische Untersuchungen bieten eben einen Zugang zum Definitionsversuch des Spielbegriffs. Das Wort „Spiel“ stammt vom alt- bzw. mittelhochdeutschen Wort „Spil“ ab, das mit einer kreisenden Tanzbewegung verbunden ist (vgl. Klippel 1980b, S. 36).

Es gibt auch andere Wissenschaftlerinnen und Wissenschaftler, die sich mit dem Vergleich der Bedeutungen des Wortes "Spiel“ in verschiedenen Sprachen auseinandersetzen. Kilp (2003, S. 92) deutet an, dass das Wort „Spiel“ auf Deutsch sehr unpräzise ist, negativer als in anderen Sprachen besetzt und mit dem Nichtstun verbunden ist. Kleppin (1989, S. 185) vergleicht das Wort „Spiel“ mit „game" und „play“ auf Englisch und findet heraus, dass, wenn man das Wort 
„Spiel“ ins Englische übersetzt, muss man sich entscheiden, ob man damit „play“ oder "game“ meint. Während "play“ freies Spiel bedeutet, ist „game“ ein regelgeleitetes Spiel.

Um Spiel genau zu definieren, ist eine Abgrenzung zwischen Spiel und NichtSpiel nötig. Man versucht, das Spiel vom Nicht-Spiel mit bestimmten Merkmalen abzugrenzen. „Der Altmeister der Spielpädagogik“ (Grätz 2001, S. 5) Hans Scheuerl liefert ein umfassendes Konzept zu Merkmalen des Spiels, das von Spielforschenden in der Fremdsprachendidaktik oft als Beispiel genommen wird (vgl. Klippel 1980b, S. 40-45; Mátyás 2009, S. 90-92). Scheuerl (1991, S. 203206) versucht, dem Spielbegriff eine phänomenologische Klärung mit sechs Merkmalen zu geben (siehe Abbildung 3).

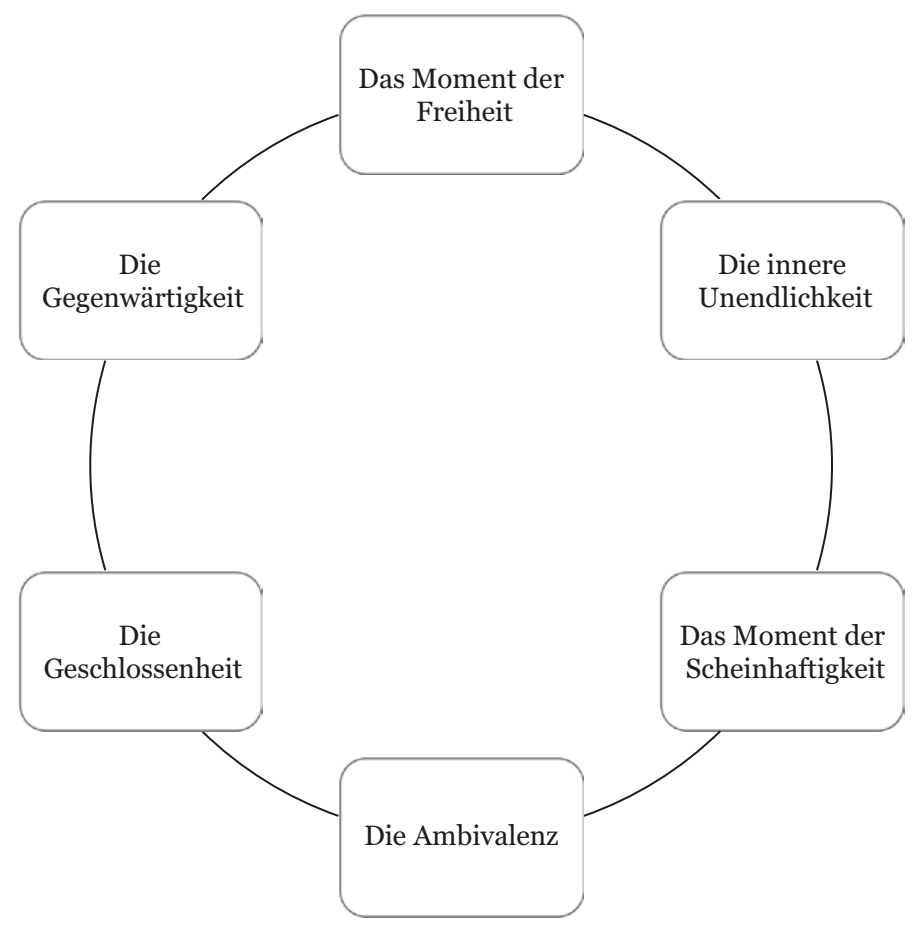

Abbildung 3: Die sechs Merkmale des Spiels im Scheuerls Konzept

Hier muss man aber fragen: Kann man mit den Merkmalen ein Spiel vom Nicht- Spiel abgrenzen? Ist Spiel ein objektives Phänomen, oder ist ein Spiel vom Spieler subjektiv hervorgebracht? Klippel (1980b, S. 88) schreibt zu den Fragen: 
Die Grenzen zwischen Spiel und Nicht-Spiel liegen [...] nicht vorwiegend in der Natur der

Tätigkeit, sondern in der Einstellung des Spielenden.

Moderne Spieltheoretiker sprechen dafür, dass jede Tätigkeit sowohl Arbeit als auch Spiel sein kann (vgl. Kleppin 1989, S. 185). Klippel (1980b, S. 39) empfiehlt, dass eine allgemein gültige Definition zur Untersuchung spezieller Spielformen keine unabdingbare Voraussetzung darstellt, weil die Frage keine unmittelbaren Auswirkungen auf die Beschäftigung mit unterrichtlichen Spielformen hat. Auch andere Forschende teilen die gleiche Position (vgl. Koenig 2003, S. 10; Jentges 2007, S. 21).

Daher wird hier auf eine weitere Auseinandersetzung zur Definition zum Spiel im allgemeinen Sinn verzichtet. Allerdings ist eine Begriffsklärung und Abgrenzung vom Sprachlernspiel im Fremdsprachenunterricht für die Durchführung der empirischen Forschung von Bedeutung. Im Folgenden soll geklärt werden, was unter dem Begriff Sprachlernspiel in der vorliegenden Forschung im Rahmen der Fremdsprachendidaktik verstanden werden kann.

\subsection{Zur Definition von Sprachlernspielen im Fremdsprachenunterricht}

Wie bei der Definition vom Begriff Spiel im allgemeinen Sinn, gibt es in der Fachliteratur noch keine einheitliche Definition oder Bezeichnung zum Spieleinsatz im Fremdsprachenunterricht.

Obwohl Sprachlernspiele seit den achtziger Jahren des letzten Jahrhunderts in einigen wissenschaftlichen Veröffentlichungen so präsent sind, dass sie als fester Bestandteil des Fremdsprachenunterrichts gesehen werden können, herrscht im Bereich der Einordnung und Terminologie Divergenz (vgl. Jentges 2007, S. 21). Kleppin (2017, S. 325) weist darauf hin, dass Sprachlernspiele zur Zeit der kommunikativen Wende einen besonderen Stellenwert einnahmen und heute dem Unterrichtsprinzip aufgabenorientierten Lernens zugeordnet werden können.

Im Folgenden wird versucht, zuerst einen Überblick über die Einordnung vom Sprachlernspiel im Lernkontext der Fremdsprachendidaktik zu verschaffen. Danach wird eine Abgrenzung zwischen Sprachlernspielen und Spielen im allgemeinen Sinne sowie zwischen Sprachlernspielen und anderen Arbeitsund Übungsformen im Fremdsprachenunterricht gemacht, um zu veranschaulichen, wie der Begriff Sprachlernspiel in der vorliegenden Arbeit verstanden werden kann. 


\subsubsection{Die Terminologien und die Zuordnung der Sprachlernspiele}

Manche Forschende sind der Meinung, dass ein Spiel im Unterricht eine Art von Arbeits- oder Übungsform ist (vgl. Rösler 1994, S. 126; Behme 1985b, S. 10; Kilp 2003, S. 94; Bohn und Schreiter 2001, S. 419f.). Manche ordnen Spiel dem Oberbegriff Tätigkeit (vgl. Klippel 1980a, S. 39) oder Aktivität (vgl. Steinhilber 1986, S. 6) zu.

Kilp (2003, S. 91) verweist darauf, wie uneinheitlich die Verwendung des Begriffs „Spiel“ im Unterricht ist: „es ist festzustellen, dass es keine einheitliche Definition für das Spiel, geschweige denn für das Lern- und Sprachlernspiel gibt.“

Begriffe wie „Unterrichtsspiel“ (Steinhilber 1986), „communicative activities“ (Klippel 2010), „Lernspielmaterialien“ sowie „Lernspiel“ (Klippel 1980a) und „Sprechspiel“ (Behme 1985a) tauchen in verschiedenen Spielsammlungen sowie Spielforschungen auf.

Um die Begriffe zu definieren, versuchen manche Autorinnen und Autoren, die Begriffe zur Gruppe zu ordnen.

Kilp (2003, S. 93) spricht sich dafür aus, dass der Begriff Sprachlernspiel als Oberbegriff von allen Spielen, die im Sprachunterricht und Fremdsprachenunterricht eingesetzt werden, verstanden werden soll.

Bohn und Schreiter (2001, S. 419f.) benutzen den Begriff "Sprachlernspiel“ und zählen ihn zu der „umfangreichen und vielfältigen Gruppe der Lernspiele“. Lernspiel wird als Synonym zum „didaktischen Spiel“ verstanden und unter dem Oberbegriff „Übungen mit spielhafter Handlungen“ dem Fremdsprachenunterricht zugeordnet (siehe Abbildung 4).

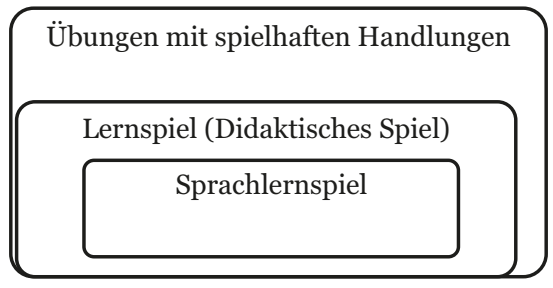

Abbildung 4: Zuordnung vom Sprachlernspiel von Bohn und Schreiter (vgl. Bohn und Schreiter 2001, S. 419f.) 
Stellfeld (1995, S. 61f.) erörtert, dass man unter „didaktischem Spiel“ nicht nur Spiele im Unterricht sondern auch Spiele im Freizeitbereich verstehen kann. Er bevorzugt deswegen in seiner Forschung eher den Begriff „Unterrichtsspiel“.

Mátyás (2009) geht auch davon aus und gibt einen Überblick von der hierarchischen Beziehung zwischen dem didaktischen Spiel, dem Unterrichtsspiel und dem Sprachlernspiel. In ihrer Abbildung (siehe Abbildung 5) sieht man, dass das Sprachlernspiel eine Form des Unterrichtsspiels ist, das besonders im Fremdsprachenunterricht eingesetzt wird.

Es ist nötig, hier an dieser Stelle noch den Unterschied zwischen Sprachspiel und Sprachlernspiel zu verdeutlichen, obwohl beide Begriffe manchmal als Synonyme benutzt werden (vgl. Kleppin 1989, S. 185):

Ein Sprachspiel ist nicht immer ein Lernspiel. Es verwendet die Sprache als Spielelement (vgl. Kilp 2003, S. 93) und bezieht sich hauptsächlich auf den kreativen Umgang mit einer Sprache (vgl. Mátyás 2009, S. 93). Kleppin (1989, S. 185) deutet an, dass der Begriff Sprachspiel sprachphilosophisch belegt ist.

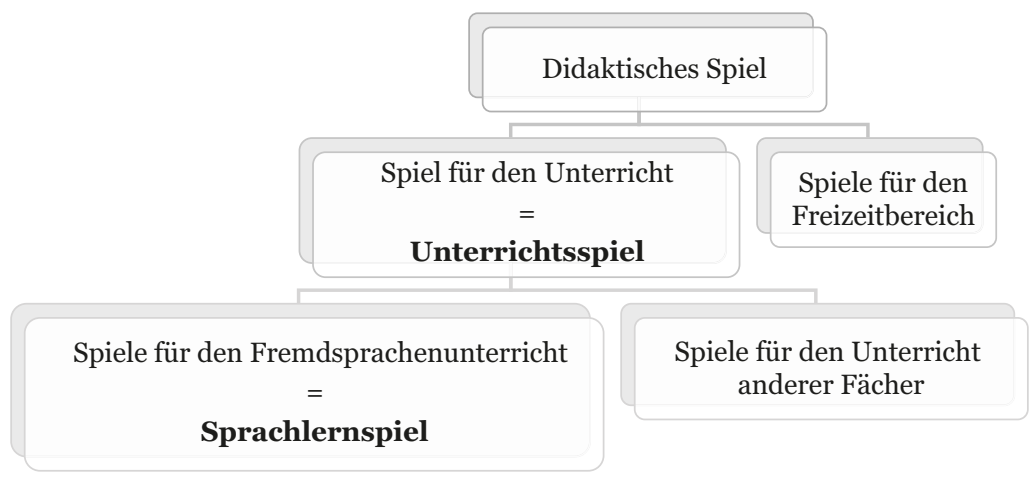

Abbildung 5: Hierarchische Beziehung zwischen den Begriffen didaktisches Spiel, Unterrichtsspiel und Sprachlernspiel (vgl. Mátyás 2009, S. 93)

In der vorliegenden Forschung wird das Wort Sprachlernspiel benutzt, da die anderen Begriffe entweder zu weit (z. B. Unterrichtsspiel, didaktisches Spiel) oder zu eng (z. B. Sprechspiel) gefasst sind.

Da weder die Einordnung des Sprachlernspiels unter den didaktischen Fachbegriffen noch die Abgrenzung zwischen Sprachspiel und Sprachlernspiel nachgewiesen werden kann, und was genau ein Sprachlernspiel ist und welche Einsatzformen dem zugeordnet werden können, ist im Folgenden eine nähere 
Auseinandersetzung bezüglich Sprachlernspiel, Spiel im allgemeinen Sinn und andere Übungsformen zu finden. Dadurch wird veranschaulicht, was in der vorliegenden Arbeit unter dem Begriff Sprachlernspiel genau verstanden wird.

\subsubsection{Abgrenzung und Gemeinsamkeiten von Sprachlernspielen und Spiel}

„Die Grenzen zwischen dem freizeitlich- unterhaltenden Beschäftigungsspiel und dem Lernspiel sind meiner Meinung nach fließend." So schrieb Behme (1985b, S. 12). Um die Fragen, ob Sprachlernspiel ein Spiel ist und was der Unterschied zwischen Sprachlernspiel und Spiel ist, zu beantworten, wird hier versucht, die gemeinsamen Merkmale und die Unterschiede zwischen Sprachlernspiel und Spiel im allgemeinen Sinn aufzulisten.

Davor wird zuerst das im Kapitel 3.1 erwähnte Scheuerlkonzept zu den Merkmalen des Spiels näher bestimmt. Scheuerl listet sechs Merkmale des Spiels auf: die Freiheit, die innere Unendlichkeit, die Scheinhaftigkeit, die Ambivalenz, die Geschlossenheit und die Gegenwärtigkeit (vgl. Scheuerl 1991, S. 203-206). Die folgende Zusammenfassung von Interpretationen der genannten Merkmale in der Tabelle 3 dient als Ausgangspunkt für die Annährung der Definitionen von Spiel und Sprachlernspiel.

Mit den sechs Merkmalen kann man unter Spiel verstehen, dass eine Tätigkeit bezeichnet wird, die ein besonderes Zeitsystem hat, die Freude spendet, sodass man den Verlauf der Tätigkeit genießen und die Wirklichkeit vergessen kann. Die Freiheit, die Gegenwärtigkeit und die Scheinhaftigkeit erlauben einem, sich im Spiel ganz frei und ohne Angst zu verhalten, weil die Handlungen im Spiel keine Konsequenzen für die Wirklichkeit haben. Spiele im Unterricht einzusetzen kann deswegen eine angstfreie und motivierende Atmosphäre realisieren, in der die Teilnehmer sich frei äußern und mit Freude lernen können. 
Tabelle 3: Die sechs Merkmale eines Spiels vom Scheuerl und deren Interpretationen

Merkmal

Die innere Unendlichkeit

Das Moment der Scheinhaftigkeit

Die Ambivalenz

Die Geschlossenheit

Die Gegenwärtigkeit

\section{Bedeutung des Merkmals}

Die Bedeutung dieses Merkmals variiert von Autor zu Autor (vgl. Klippel 1980b, S. 40). Bei manchen Autoren wird auch Zweckfreiheit genannt, z. B. Steinhilber (1986, S. 17). Das Merkmal wird häufig als zentrales Merkmal des Spiels verstanden (vgl. Klippel 1980b, S. 40). Scheuerl (1994, S. 67) sieht das Merkmal als „Eines der hervorstechendsten Merkmale des Spiels“. Klippel fasst so zusammen: „für Scheuerl ist das Spiel Selbstzweck für den Spielenden“" (Klippel 1980b, S. 40).

Dieses Merkmal bedeutet, dass die Spieler dazu tendieren, das Spiel so lange wie möglich zu führen oder zu wiederholen (vgl.ebenda, S. 41). So Scheuerl (1991, S. 205): „ Das Ende (eines Spiels) kommt jedes Mal durch Mächte, die für die Spielbewegung selber äußerlich sind: durch den Ruf der Mutter, durch Hunger, Müdigkeit, Ungeduld, alterseigentümlicher Zappeligkeit - jedenfalls nicht aus der gestalthaften Verlaufsform des Spiels selber."

Im Spiel vergisst der Spieler das reale Leben. Es ist möglich, fiktiv zu handeln (vgl.Scheuerl 1994, S. 77-85; Klippel 1980b, S. 41).

Kennzeichnung der Spielspannung, die weder zu gering noch zu groß sein darf, sodass der Spieler sich nicht gelangweilt oder überfordert fühlt (vgl. Klippel 1980b, S. 43; Mátyás 2009, S. 91; Scheuerl 1991, S. 204).

Das Spiel hat eine räumliche und zeitliche Grenze, folgt bestimmten Regeln und Normen (vgl. Scheuerl 1991, S. 204; Klippel 1980b, S. 43f.; Mátyás 2009, S. 91).

Der Spielverlauf ist wichtiger als das Resultat. Die Aufmerksamkeit des Spielendes ist auf die Gegenwart in der Spielzeit gerichtet (vgl. Mátyás 2009, S. 92; Klippel 1980b, S. 44; Scheuerl 1994, S. 95-102). 
Ein Spiel im Fremdsprachenunterricht, also ein Sprachlernspiel, hat noch einige besondere Merkmale gegenüber Spiel im allgemeinen Sinn. Mátyás vergleicht in ihrer Forschung das Scheuerlkonzept mit den Charakteristika des Sprachlernspiels (siehe Abbildung 6), die von Kleppin (1980, S. 38-40) zusammengestellt wurden, und kommt zu folgendem Resultat:

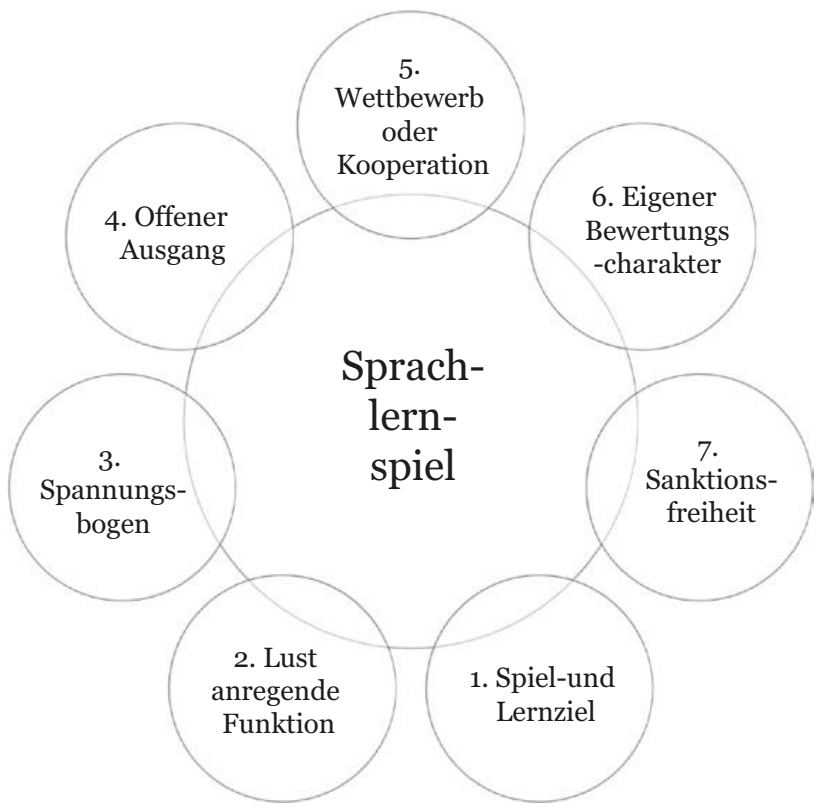

Abbildung 6: Kriterien des Sprachlernspiels nach Kleppin von Mátyás (2009, S. 94)

Mátyás (2009, S. 101) kommt zum Schluss, dass die Liste der Kleppin'schen Charakteristika vom Sprachlernspiel sich größtenteils mit den theoretischen Überlegung des Scheuerl-Spielkonzepts decken. Mit dem Vergleich dieser zwei Listen vom Merkmalen stellt Mátyás fest, dass der wichtigste Unterschied zwischen Sprachlernspiel und Spiel darin besteht, dass Sprachlernspiel außer Spielziel noch ein Lernziel hat (vgl. ebenda, S. 94). Ein Spiel soll nach dem Merkmal der Freiheit von Scheuerl zweckfrei sein. Aber der Einsatz eines Sprachlernspiels ist immer zweckgebunden (vgl. Jentges 2007, S. 23). So Meyer (1994, S. 344):

Spielen im Unterricht ist nicht zweckfrei, sondern ein zielgerichteter Versuch zur Entwicklung der sozialen, kreativen, intellektuellen und ästhetischen Kompetenzen der Schüler. 
Mit der Zweckgebundenheit des Sprachlernspiels geht das Freiheit-Merkmal eines Spiels teilweise verloren. Allerdings sollen die Teilnehmer das Sprachlernspiel während eines Spielverlaufs subjektiv zweckfrei erleben, damit sie den Eindruck haben, dass sie nicht um des Lernens willen, sondern um des Spielens willen das Sprachlernspiel spielen. So kann eine völlige Konzentration auf das Spiel erst erfolgen (vgl. Mátyás 2009, S. 95).

Zusammenfassend lässt sich sagen, dass einige Merkmale, die für Spiele im allgemeinen Sinne typisch sind, für Sprachlernspiele in unterschiedlichem Maße charakteristisch sind (vgl. ebenda, S. 101):

Tabelle 4: Gegenüberstellung des Kleppin-Konzepts zum Scheuerlkonzept nach Mátyás (2009, S. 101f.)

\begin{tabular}{|c|c|c|}
\hline \multicolumn{2}{|c|}{ Sprachlernspiel Charakteristika von Kleppin } & \multirow{2}{*}{$\begin{array}{l}\text { Gekoppeltes } \\
\text { Merkmal vom } \\
\text { Scheuerlkonzept }\end{array}$} \\
\hline Charakter & Bedeutung & \\
\hline Spannungsbogen & $\begin{array}{l}\text { „Der Wechsel zwischen dem Anwachsen } \\
\text { und Nachlassen der Spielsammlung, was } \\
\text { die Spielenden zur Fortsetzung des Spiels } \\
\text { motiviert.“ (Mátyás 2009, S. 97) }\end{array}$ & Die Ambivalenz \\
\hline Eigener & Das Spiel hat wegen vereinbarter & Die Geschlossenheit \\
\hline Bewertungscharakter & $\begin{array}{l}\text { Spielregeln ein eigenes Bewertungssystem } \\
\text { und schafft damit die Möglichkeit, selbst zu } \\
\text { evaluieren (vgl. ebenda, S. 100). }\end{array}$ & \\
\hline Die Sanktionsfreiheit & $\begin{array}{l}\text { Ein Sprachlernspiel soll nicht von der } \\
\text { Lehrperson bewertet werden (vgl. ebenda, } \\
\text { S. 100). }\end{array}$ & $\begin{array}{l}\text { Die Gegenwärtigkeit } \\
\text { Die Scheinhaftigkeit }\end{array}$ \\
\hline
\end{tabular}

Die Kriterien des Sprachlernspiels nach Kleppin, die vom Scheuerlkonzept fürs Spiel im Allgemeinen Sinne nicht ausgeschlossen werden, sind die Lust anregende Funktion, der offene Ausgang und der Wettbewerbscharakter des Sprachlernspiels.

Laut Mátyás (2009, S. 96) soll ein Sprachlernspiel so konzipiert werden, dass die Teilnehmer ein freudiges Erlebnis haben können. Nur dadurch kann die motivierende Funktion einer Spieltätigkeit wirken. Außerdem muss das Sprachlernspiel auch offen sein, sodass man den Verlauf und das Ergebnis nicht genau vorhersagen kann und Spannung und Freude im Spiel entstehen können (vgl. ebenda, S. 98). Ein angemessener Wettbewerbscharakter bietet im Spielverlauf Kooperationsmöglichkeiten zwischen Gruppen, verschafft Interaktionen, fördert Offenheit des Spielausgangs. Deswegen sollen die Elemente Wettbewerb 
und Kooperation einen festen Platz nebeneinander in dem Spiel haben (vgl. ebenda, 100-103).

Zusammenfassend lässt sich sagen, dass ein Sprachlernspiel ein Spiel ist, das von DidaktikerInnen und Lehrenden aus einem Spiel im allgemeinen Sinn für Fremdsprachenlernende adaptiert wird (vgl. Dauvillier und Meese 2004, S. 18). Ein Sprachlernspiel hat das Erlernen einer Sprache als Zweck und kann als eine Art von Arbeits- und Übungsform im Fremdsprachenunterricht verstanden werden (vgl. Kilp 2003, S. 93f.). Im Folgenden wird veranschaulicht, wie sich ein Sprachlernspiel von anderen Arbeits- und Übungsformen unterscheidet.

\subsubsection{Abgrenzung und Gemeinsamkeiten von Sprachlernspielen und anderen Arbeits- und Übungsformen}

Nach Kilp (2003, S. 93f.) kann das Sprachlernspiel als Extremform von Übungen verstanden werden. Aber was ist eine Übung? Wohin kann man das Sprachlernspiel im Kontext des Fremdsprachenunterrichts einordnen? Hier wird versucht, Antworten auf diesen Fragen zu liefern.

Jentges (2007, S. 21) deutet an, dass eine Auseinandersetzung mit Grundbegriffen des Unterrichts nötig ist, um diese Fragen zu beantworten. Bevor das Sprachlernspiel als Übungs- oder Aufgabenform definiert wird, werden hier an erster Stelle die Grundbegriffe zur Übungsform erklärt.

Wie bei den Begriffen Spiel und Sprachlernspiel, gibt es in der Fachliteratur ebenso kaum eine endgültige einheitliche Definition für den Begriff Übung:

Die Terminologie, mit der die Vorlagen bezeichnet werden, an denen sich Üben vollziehen soll, ist unscharf und widersprüchlich. Das Verständnis von Übungsformen, Übungstyp und schließlich Übungstypologie wird von jedem Autor unterschiedlich gedeutet. [...] In jeder fremdsprachendidaktischen ,Epoche' waren Autoren bestrebt, Übungsformen und Übungstypen zu einer sinnvollen, unmittelbar einsetzbaren Übungstypologie zu vereinigen (Schwerdtfeger 1989, S. 188).

Auch wenn die Autoren sich nicht darauf verständigen können, was man unter Übung genau verstehen kann, sind sie sich doch darin einig, dass die Übung für Fremdsprachenunterricht ein enorm wichtiger Bestandteil des Lernprozesses ist und als zentrale Phase betrachtet werden sollte (vgl. Rösler 1994, S. 126; Schwerdtfeger 1989, S. 187; Huneke und Steinig 2010, S. 229; Kieweg 2010, S. 182).

Rösler (1994, S. 127) ordnet Übung als Arbeitsform ein und greift auf die Definition der Übung von Schwerdtfeger zurück: 
Übung ist eine Handlung des Lernenden, in deren Verlauf er identische oder ähnliche Sachverhalte wieder und wieder lernt, um sie zu behalten und für den eigenen produktiven Umgang zur Verfügung zu haben (Schwerdtfeger 1989, S. 187f.).

Schwerdtfeger (1989, S. 187f.) weist noch den Unterschied zwischen Arbeitsform und Übungsform nach: Arbeitsform beschreibt ein umfassenderes und generelleres unterrichtliches Geschehen als der Begriff Übungsform, und obwohl beide Begriffe oft synonym verwendet werden, ist der Begriff Übungsform spezieller. Sie ist der Meinung, dass eine Übung die Funktion hat, die Stabilität und Deutlichkeit des Gelernten in der kognitiven Struktur zu verstärken und den Grad ihrer Dissoziierbarkeit und ihrer Bewahrung im Gedächtnis zu erhöhen.

Huneke und Steinig (2010, S. 229f.) finden, dass die Übung als Abschlussphase im Lernprozess angesehen werden soll und eine Voraussetzung für die Anwendung des gelernten Wissens bildet. Sie stützen sich darauf, dass eine erfolgreiche Übung 3 Merkmale besitzt: Wiederholung, Variation und Motivation. Die drei Merkmale sind miteinander fest verknüpft. Wiederholen dient als das grundlegende Element des Übens. Man wiederholt das Gelernte mehrmals, bis man davon überzeugt ist, dass man das Gelernte richtig beherrscht. Das heißt aber nicht, dass jede Wiederholung mit der anderen identisch sein muss. Nur wenn beim Üben eine gewissen Variation von Übungen zur Verfügung steht, kann eine Wiederholung effektiv sein, weil es motivierender ist. Nur wenn das Üben für einen Lernenden motivierend ist, kann die Übung erfolgreich sein (siehe Abbildung 7).

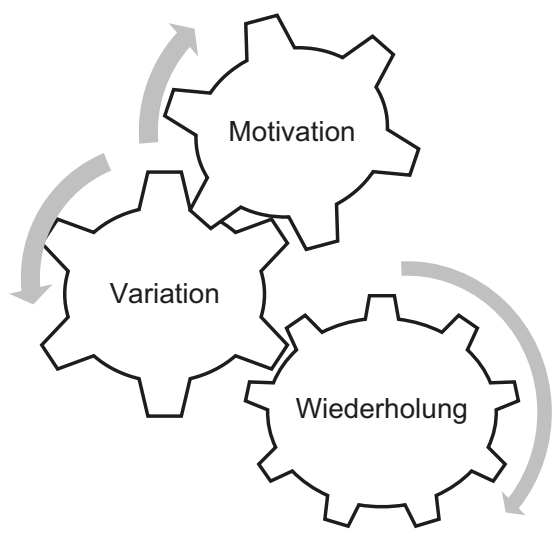

Abbildung 7: Die miteinander verknüpften Merkmale 
Man kann feststellen, dass es nicht leicht ist, eine Übung erfolgreich durchzuführen. Für Lehrende ist es eine Herausforderung, eine Übung für die Lernenden motivierend und wirksam zu konzipieren. Dazu schreibt Rösler (1994, S. 127):

Dass Übungen oft als langweilig empfunden werden und oft recht eintönig sind, hat eher mit der mangelnden Phantasie der Übungsmacher zu tun oder mit ihrem Glauben daran, dass in einem bestimmten Ansatz nur bestimmte Übungen zulässig seien, als damit, dass es an Beschreibungen einer möglichen Übungsvielfalt fehlt.

Übungen sind sprachbezogen, behandeln ein sprachliches Phänomen, und betonen den Einsatz von vorab festgelegten Strukturen, Redemitteln oder Fertigkeiten. Sie sind nicht mitteilungsbezogen (vgl. Huneke und Steinig 2010, S. 232; Klippel 2010, S. 186; Dauvillier und Meese 2004, S. 12).

Während eine Übung stark lenkend ist, eine eindeutige Lösung und einen vorgegebenen Lernweg hat (vgl. Dauvillier und Meese 2004, S. 12), ist eine Aufgabe offener, freier und kommunikativ (vgl. Dauvillier und Meese 2004, S. 14; Huneke und Steinig 2010, S. 232). Obwohl es fließende Grenzen zwischen den verschiedenen Arbeitsformen gibt, kann man eine Reihe von Unterschieden in der Übung und der Aufgabe feststellen, so Huneke und Steinig (2013):

Tabelle 5: Die Prototypische Gegenüberstellung von Übung und Aufgabe (Huneke und Steinig 2013, S. 233)

\begin{tabular}{ll}
\hline Übung & Aufgabe \\
\hline $\begin{array}{l}\text { Ziel: korrekte Sprachverwendung; } \\
\text { sprachbezogen }\end{array}$ & $\begin{array}{l}\text { Ziel: Gelingen von Mitteilung und Verstehen } \\
\text { bei der Kommunikation in der Lerngruppe; } \\
\text { mitteilungsbezogen }\end{array}$ \\
im Unterrichtsprozess entstanden \\
vom Lehrer erstellt & Lösungswege müssen von den Lernern \\
ein vorgeplanter Lösungsweg & gefunden werden \\
eine richtige Lösung & mehrere Lösungen möglich \\
fordert und fördert Orientierung an der & fordern und fördern Autonomie \\
(sprachlichen) Norm & süd dem Übungsgeschehen \\
haben dienende Funktion: Erleichterung der & übergeordnet: erfordern Übungen, um \\
Lösung von Aufgabe & leichter lösbar zu sein \\
& tendenziell: „Offenheit“ \\
\hline tendenziell: , Geschlossenheit“ &
\end{tabular}

Die Gegenüberstellung verdeutlicht, dass die Offenheit einer Arbeitsform für die Abgrenzung zwischen Übung und Aufgabe am wichtigsten ist. Die Offenheit der Aufgabe betrifft drei Faktoren: die Aufgabestellung, die Lösungswege und das Ergebnis. Für die Lernenden heißt dies, dass sie in einer Aufgabe einen 
individuelleren und kreativeren Lernprozess erleben können. Bei der Lösung einer Aufgabe können sie selbstständiger mit der Zielsprache umgehen. Sie können das Vorgegebene antizipieren, verändern, umstrukturieren, ausweiten und die Perspektive wechseln. Eine offene und kreative Aufgabe kommt dem Sprachlernspiel nahe (vgl. Dauvillier und Meese 2004, S. 15f.).

Kilp (2003, S. 94) versteht das Sprachlernspiel als eine Extremform der Übung, die den höchsten Freiheitsgrad bei allen Übungsformen hat. Rösler (1994, S. 133) sieht Spiele im Fremdsprachenunterricht als unterschiedliche Aktivitäten, die klein und zeitlich sehr begrenzt sind. Sie werden als Übungen zielgerichtet eingesetzt. Klippel (2010, S. 187) schließt sich dieser Meinung an und akzeptiert Sprachlernspiele als eine „Teilmenge von Activities und Übungen“.

Die Grenze zwischen Sprachlernspiel und anderen Übungsformen ist fließend (vgl. Schweckendiek 2001, S. 11). Das hat einerseits mit subjektiver Wahrnehmung zu tun (vgl. Klippel 2010, S. 188), was im Kapitel 3.1 zum Spiel diskutiert wird. Andererseits ist man auch davon überzeugt, dass viele als Spiel bezeichnete Arbeitsformen im Fremdsprachenunterricht eigentlich traditionellen Übungsformen entsprechen. Es ist die Art und Weise, wie die Übung, Aufgabe oder Aktivität durchgeführt werden. Damit wird bestimmt, ob eine Arbeitsform ein Spiel im Unterricht ist. Sprachlernspiel ist kein bestimmter Übungstyp, keine Methode, sondern das Wie einer Übung, einer Aufgabe oder einer Aktivität, die im Fremdsprachenunterricht durchgeführt wird (vgl. Jentges 2007, S. 22f.).

\section{In der vorliegen Arbeit werden Sprachlernspiele so verstanden:}

Ein Sprachlernspiel ist eine, von Didaktikern und Lehrenden aus einem Spiel oder anderen Arbeits- und Übungsformen, fürs Lernen adaptierte kreative Aufgabenstellung. Sie vermittelt und festigt die Lerninhalte auf spielerische Weise. $\mathrm{Zu}$ Sprachlernspielen gehören alle Aktivitäten, die in einem handlungsorientierten, kommunikativen und Lernautonomie fördernden Unterricht sind, die auch wie „spielerische Übungen und Aufgaben“ bezeichnet werden (vgl. Dauvillier und Meese 2004, S. 18f.).

Sprachlernspiele können von jeder Übung, Aufgabe oder Aktivität adaptiert werden, weil die Art und Weise der Durchführung entscheidender ist als der Inhalt. Sprachlernspiele sollen der Mehrheit der genannten Charakteristika von Kleppin (Kapitel 3.2.2) entsprechen, außer dem „offenen Ausgang“. Es gibt nämlich Spiele wie Kreuzworträtsel, die für Fremdsprachenunterricht gut geeignet sein können, aber nur eine richtige Antwort haben. Außerdem werden Rollenspiele in der vorliegenden Arbeit in Anlehnung von Klippel (2010, S. 189) nicht als Sprachlernspiele betrachtet, weil sie keine Regelspiele bzw. „games“ sind, sondern "play“. Außerdem ist zu bemerken, dass subjektive Wahrnehmung 
dabei immer eine Rolle spielt, was man unter Spiel versteht. Kilp (2003, S. 94) schreibt dazu:

Was für Kinder ein Spiel ist, kann für Erwachsene lediglich eine Übung mit spielerischem Charakter sein. Übungen, die für Kinder Spiele darstellen, müssen für Erwachsene nicht die gleiche Bedeutung haben.

\subsection{Die Effekte der Sprachlernspiele}

In diesem Kapitel geht es um die Diskussion der erwarteten Effekte bzw. Funktionen von Sprachlernspielen im Fremdsprachenunterricht.

Um die Effekte von Sprachlernspielen besser zu verstehen, werden im Vordergrund die Funktionen des Spiels im Allgemeinen diskutiert. Danach werden die möglichen Effekte von Sprachlernspielen beschrieben.

\subsubsection{Die Funktion von Spielen im allgemeinen Sinn}

Seit langer Zeit diskutiert man über das Spiel, über dessen Definition, Klassifikation und Funktionen. Das Spiel ist ein vielschichtiges und komplexes Phänomen. Deswegen ist bis heute keine einheitliche Definition gelungen (vgl. Döring 1997, S. 59; Klippel 1980b, S. 38; Steinhilber 1986, S. 6; Jentges 2007, S. 21; Kreuzer 1983, S. 7). Keine Spieltheorien oder Denkmodelle reichen aus, das spielerische Verhalten zu erklären (vgl. van der Kooij 1983, S. 303).

Erst im 17. und 18. Jahrhundert wird das Spiel von den Philosophen als notwendige Entwicklungsphase von Kindern erkannt (vgl. ebenda, S. 297f.). Schiller schreibt in seinem fünfzehnten Brief über die ästhetische Erziehung einen oft zitierten Satz (vgl. Scheuerl 1983, S. 34; Koenig 2003, S. 11; Hansen und Wendt 1990, S. 15): „Der Mensch spielt nur, wo er in voller Bedeutung des Wortes Mensch ist, und er ist nur da ganz Mensch, wo er spielt." (Schiller 1991, S. 37).

Heute ist man sich darin einig, dass Spiele kognitive, emotionale, soziale und kreative Fähigkeiten fördern und motivierend sein können (vgl. Döring 1997, S. 28; Warwitz und Rudolf 2004, S. 82). Spiele können als ein Lernprozess verstanden werden. Zu der Frage „Warum spielen Kinder?“ schreibt Spitzer (2000, S. 62), dass manche Verhalten, wie Kampfverhalten z. B., nur erlernt werden können, wenn sie ohne Schaden für den Organismus immer wieder geübt werden können. Im Spiel kann risikoarmes Lernen stattfinden. Man erwirbt im Spiel Kompetenzen, die zur Bewältigung der Konflikte und Aufgaben im Leben notwendig sind (vgl. Oerter 1997, S. 283). 
Scheuerl (1983, S. 33) erörtert, dass Spiele in der Gesamtheit menschlicher Aktivitäten und Erlebnisweisen bei Kindern, Jugendlichen und Erwachsenen einen sehr unterschiedlichen Stellenwert haben.

Für das Kind ist das Spiel ein Medium, in dem sich kognitive und emotionale Anteile vereinen, wo Emotionen deutlich ausgedrückt werden können, und gleichzeitig die Realität nachgespielt und umgestaltet werden kann (vgl. Oerter 1997, S. 263).

Im Erwachsenenalter transformiert sich das kindliche Spiel auf eine andere Ebene. Spiele werden in andere Tätigkeiten integriert und institutionalisiert. So leben Erwachsene ihren Spieltrieb z. B. bei öffentlichen Wettkämpfen und sportlichen Spielen aus. Dabei können Preise gewonnen und sogar Karriere gemacht werden, etwa beim Fußball oder Tennis. „Spiele“ finden auch im privaten Bereich statt; sie werden dann als Hobbys bezeichnet und dienen der Entspannung und dem Zeitvertreib. Erwachsene spielen also anders, aber dennoch mit ähnlichen Zielen wie Kinder (vgl. ebenda, S. 310-315). Van der Kooij (1983, S. 324) schließt sich auch dieser Meinung an:

Der Erwachsene tritt ins Spiel ein als in eine durch ihn geschaffene Scheinwelt, die der Realität der Arbeitswelt gegenübersteht. Das Kind hingegen macht im Spiel Erfahrungen zum Zwecke einer besseren Beherrschung der Wirklichkeit.

Zusammenfassend lässt sich sagen:

1. Das Spiel ist eine Form des risikoarmen Lernens, dass Kinder auf das spätere Sozialleben vorbereiten kann. Kinder lernen im Spiel nötige Fertigkeiten zum Bewegen, Denken und gesellschaftlichen Leben. Den Erwachsenen dient Spiel hauptsächlich als Erholung und Entspannung von der Arbeit und zweckgerichtetem Leben.

2. Im Spiel werden Emotionen angeregt, die den psychischen Zustand von Kindern und Erwachsenen beeinflussen können. Spiele sind Lust anregend und Angst abbauend.

Warwitz und Rudolf (2004, S. 83) heben hervor, dass Spiele neue Dimensionen fürs Lernen eröffnen können:

Es schafft befreite, offene Situationen, die es ermöglichen, sich aktiv und schöpferisch mit Problemen und Aufgaben auseinanderzusetzen[...]Spielendes Lernen eröffnet die Chance, selbstständig zu experimentieren, originelle Lösungen zu finden und kreativ zu sein. Es nutzt zudem die Motivationskraft des Spielens. Beim spielerischen Lernen wird Denken als Probehandeln, als Auseinandersetzung mit eigenen Möglichkeiten und Grenzen herausgefordert... 
Infolgedessen kann man vermuten, dass Spiele für Kinder und auch für Erwachsene wichtig sind und eine Chance fürs Lernen bieten können. Im Folgenden werden die Effekte von Spielen im Fremdsprachenunterricht unter die Lupe genommen.

\subsubsection{Effekte von Sprachlernspielen im Fremdsprachenunterricht}

Groos (1991, S. 70) gibt Aufschluss über das Erwerben von Sprache:

Teils ohne Spielstimmung, zum Teil aber auch in spielender Betätigung [...] erwirbt ein jedes Kind durch Nachahmung den köstlichsten Kulturschatz eines Volks, die Muttersprache. Es erlernt sie von selbst - und viele unter den großen Pädagogen haben es als ein Ideal bezeichnet, den Menschen auch die Fremdsprachen in solch spielender Weise erlernen zu lassen.

Hopp $(1983,288)$ stimmt dieser Meinung zu und pointiert, dass alles sprachliche Handeln Inhalt des Spiels sein kann, weil alle Arten von Tätigkeiten des menschlichen Lebens mögliche Inhalte bzw. Bestandteile eines Spiels sein können.

Im Folgenden werden die Effekte von Sprachlernspielen im Fremdsprachenunterricht unter drei Hauptaspekten zusammengefasst, nämlich dem Aspekt von Motivation, dem Aspekt von Kommunikation und Interaktion, und dem Aspekt von Lerneffizienz und Wissensvermittlung.

\subsubsection{Aspekt der Motivation}

Die motivierende Funktion von Sprachlernspielen wird von vielen Autoren bestätigt (vgl. Hoppe 1983, 293; Koenig 2003, S. 9; Hoppe 1985, S. 7; Steinhilber 1986, S. 15f.; Dauvillier und Meese 2004, S. 24; Hansen und Wendt 1990, S. 35; Jentges 2007, S. 37; Kacjan 2008, S. 59; Bohn und Schreiter 2001, S. 421).

Mátyás (2010, S. 396) findet, dass die motivierende Funktion eine der wichtigsten Eigenschaften von Sprachlernspielen ist. Die angstabbauenden und lustanregenden Effekte des Spiel können eine ideale Lernatmosphäre schaffen. Besonders für schwache Lernende sind Spachlernspiele geeignet.

Nach Bohn und Schreiter (2001, S. 421) kann der Wettbewerbscharakter des Spiels und der Reiz des Neuen die Lernmotivation in besonderer Weise stärken.

Die extrinsischen Motivationskräfte des Sprachlernspiels wie Auflockerung, Belohnung und Entspannung können die Einstellung des Lernenden zur Fremdsprache und zum Unterricht verbessern (vgl. Kleppin 2007, S. 264; Hansen und Wendt 1990, S. 35; Hoppe 1985, S. 7; Grätz 2001, S. 6). Die intrinsische Motivationskraft bzw. die innere Endlosigkeit des Spiels führt dazu, dass der Spielende die Tätigkeit fortsetzen und wiederholen will (vgl. Hansen und Wendt 1990, S. 35; Steinhilber 1986, S. 16). 


\subsubsection{Aspekt der Kommunikation und Interaktion}

Hopp (1983, S. 291f.) analysiert die Situation des Fremdsprachenunterrichts und vergleicht sie mit der des muttersprachlichen Unterrrichts, und kommt zu folgender Zusammenfassung: Im fremdsprachlichen Unterricht gibt es keine unmittelbaren Anwendungserfordernisse der Zielsprache und der Unterricht ist vom starken Übungs-und Trainingscharakter geprägt. Die Redesituation und die Sprechhandlung ist uneigentlich und künstlich. Die praktische Anwendung, kreative Handhabung von Sprache sowie die Reflexion über situative, soziale und ästhetische Funktionen des sprachlichen Gebrauchs von Fremdsprache im Fremdsprachenunterricht können nicht mit dem muttersprachlichen Unterricht verglichen werden. Er geht davon aus, dass das Spiel eine Möglichkeit bietet, einen auflockernden und kommunikationsfreundlichen Kontext zu bauen, in dem man die isolierten Wörter und Sätze in konkrete kommunikative Situationen einbetten kann (vgl. ebenda, S. 292-295).

Bohn und Schreiter (2001, S. 425) haben folgende kommunikativen Vorteile vom Spieleinsatz im Fremdsprachenunterricht zusammengefasst:

1. Mit Spiel schafft man echte, situative Sprechanlässe

2. Spiele bringen die Lernenden in Situationen, in denen kreativsprachliches Handeln gefordert ist.

Kleppin (1980, S. 21-28) betont den interaktiven Charakter von Sprachlernspielen. Sie beschreibt die Förderung der Persönlichkeitsentwicklung, der Kooperationsbereitschaft und der Solidarität als zentrale Komponente des Spielens, und kommt zu dem Schluss, dass Sprachlernspiele Kontakte in der Klasse erleichtern und die beste Form von echter Sprachanwendung sind.

Im Spiel wird die Fremdsprache als Mittel zum Erreichen eines Zwecks gebraucht, wobei kreatives sprachliches Handeln gefordert ist, um das Ziel zu erreichen, da Spiele gemeinsame Eigenschaften mit echten kommunikativen Situationen aufweisen und Interaktion mit kommunikativer Absicht ermöglichen können (vgl. Schweckendiek 2001, S. 11; Mátyás 2009, S. 112). Spiele können das freie, regelgeleitete Sprechen imitieren (vgl. Grätz 2001, S. 6) und als authentische Kommunikationssituation betrachtet werden (vgl. Hoppe 1985, S. 9; Kleppin 2007, S. 264).

Wegen der motivierenden Funktion des Spiels ist auch zu erwarten, dass die Sprechzeit der Lernenden im Sprachlernspiel erhöht werden kann (vgl. Schweckendiek 2001, S. 11), besonderes bei schwachen Lernenden (vgl. Grätz 2001, S. 6; Mátyás 2010, S. 395). 


\subsubsection{Aspekt der Lerneffizienz und Wissensvermittlung}

Die motivierenden und kommunikativen Effekte sprechen schon dafür, dass Sprachlernspiele die Lerneffizienz im Fremdsprachenunterricht erhöhen können.

Das Argument von Hoppe (1983, S. 288), dass alles sprachliche Handeln zum Inhalt des Spiels gemacht werden kann, wird von Kleppin (2007, S. 264) bestätigt: „Mit Spielen sind unterschiedliche Lernziele zu verbinden...Sprachlernspiele sind in allen Lern- und Unterrichtsphasen, in allen Sozialformen und medienunterstützt einsetzbar.“

Sprache wird in der Spielsituation unbewusst geübt (vgl. Mátyás 2010, S. 395). Im Spiel können fremdsprachliche Strukturen wiederholt und variiert werden (vgl. Koenig 2003, S. 9), dabei wird die Übungszeit intensiver genutzt (vgl. Schweckendiek 2001, S. 11). Das Lernen ist ganzheitlich (vgl. Grätz 2001, S. 6). Das Sprachlernspiel ermöglicht unbewusstes Üben, das zur Automatisierung von Sprachabläufen führen kann (vgl. Bohn und Schreiter 2001, S. 425).

Das Sprachlernspiel bietet außerdem eine Möglichkeit, affektiv zu lernen. Die Wichtigkeit der Emotionen und ihrer Verbindung mit Kognition wird von neurobiologischer Forschung nachgewiesen (vgl. Haß 2017a, S. 45). Die beim Spiel beteiligten Emotionen verbessern den Lernfortschritt, ermöglichen eine effektive Informationsverarbeitung und langfristigere Speicherung von Informationen im Gehirn (vgl. Mátyás 2010, S. 396; Dauvillier und Meese 2004, S. 25).

Klippel (1998, S. 5) betont, dass eine entscheidende Wirkung des Spiels in der Erhöhung der Aufmerksamkeit liegt: „Während des Spiels richtet sich die Aufmerksamkeit der Lernenden zuerst auf das Spielziel und diejenigen sprachlichen Aufgaben, die es erreichen helfen." Die Konzentration kann die Fähigkeit des Gedächtnisses fördern, Informationen zu behalten. Das spricht dafür, dass ein Abwechslung und Freude bietendes Sprachlernspiel den Lernprozess positiv prägen kann (vgl. Dauvillier und Meese 2004, S. 24).

Außer sprachlicher Wissensvermittlung kann man auch mit Sprachlernspielen folgendes Wissen im Unterricht erweitern:

1. Vermittlung von kulturellem und landeskundlichem Wissen (vgl. Kleppin 1980, S. 29f., 2007, S. 264; Hansen und Wendt 1990, S. 36; Kleppin 1989, S. 186; Grätz 2001, S. 6)

2. Kommunikationskompetenzen (vgl. Jentges 2007, S. 36; Hansen und Wendt 1990, S. 33; Kleppin 1989, S. 186, 1980, S. 24, 2007, S. 264)

3. Lernstrategien (vgl. Jentges 2007, S. 36; Kleppin 2007, S. 264) 
Da die Sprachlernspiele den Lernenden helfen, kommunikative Kompetenz und Lernstrategien zu entwickeln und die Sprechzeit auszuweiten, können sie auch autonomes Lernen fördern (vgl. Hansen und Wendt 1990, S. 36; Kleppin 1980, S. 30-32; Koenig 2003, S. 9; Kleppin 2007, S. 264f.; Klippel 1998, S. 5f.).

Sprachlernspiele schaffen eine angstfreie Unterrichtsatmosphäre, reduzieren Zeit- und Notendruck, weil Sprachlernspiele die Bewertung durch die Lehrperson durch mehr Selbstbewertung und Gruppenverantwortung ersetzt (vgl. Dauvillier und Meese 2004, S. 16). Zur Lernumgebung schreibt Haß (2017a, S. 45): „Die Gestaltung einer angenehmen, entspannten und anregenden Lernumgebung hat auf den Erfolg von Lernprozessen großen Einfluss."

Sprachlernspiele bieten eine Möglichkeit, im Unterricht soziale Fähigkeiten, insbesondere die Fähigkeit zur Teamarbeit, zu verbessern (vgl. Jentges 2007, S. 36), sie fördern Selbstvertrauen der Spielenden (vgl. Kacjan 2008, S. 59) und dienen dem Zweck der Binnendifferenzierung (vgl. Mátyás 2009, S. 116; Kleppin 2007, S. 264).

Über die Multifunktionalität von Sprachlernspielen schreibt Meyer (1994, S. 344):

Spielen im Unterricht ist nicht zweckfrei, sondern ein zielgerichteter Versuch zur Entwick-

lung der sozialen, kreativen, intellektuellen und ästhetischen Kompetenzen der Schüler.

Zusammenfassend lässt sich sagen, dass Spiele im Fremdsprachenunterricht das Lernen ganzheitlich fördern können. Sprachlernspiele sollten ein fester Bestandteil des Fremdsprachenunterrichts sein. Wegen der Multifunktionalität sollen Sprachlernspiele die WTC von Fremdsprachenlernenden fördern.

Allerdings gibt es heute immer noch eine Diskrepanz zwischen Theorie und Praxis des Spieleinsatzes im Fremdsprachenunterricht. Um dieses Problem zu veranschaulichen, wird der Forschungsstand zu den Wirkungen des Sprachlernspiels im Folgenden unter die Lupe genommen.

\subsection{Forschungsstand vom Einsatz der Sprachlernspiele}

\subsubsection{Allgemeiner Forschungsstand des Spieleinsatzes im Fremdsprachenunterricht}

Den Spieleinsatz im Fremdsprachenunterricht gibt es seit Anfang der neuzeitlichen Pädagogik in Europa. J. A. Comenius berücksichtigte in seiner im Jahr 1656 veröffentlichten Schola ludus (Schule als Spiel) das Spiel ausführlich und integrierte das Lernspiel als Mittel für den Erwerb und das Festigen von fremdsprachlichen Strukturen (vgl. Hansen und Wendt 1990, S. 16; Mátyás 2009, S. 110). 
Im 19. Jahrhundert berücksichtigen pädagogische Theorie und Praxis der Philanthropen das Spiel in noch stärkerem Maße als bei Comenius, nicht zuletzt auch unter dem Einfluss der Gedanken einer Reihe bedeutender politischer Denker wie Locke und Rousseau. Dichtern wie Lessing, Schiller und Goethe sowie Philosophen wie Kant und Hegel (vgl. Klippel 1980b, S. 63; Kube 1977, S. 26f.). Laut Kube (1977, S. 26f.) galt das Spiel als Möglichkeit der Entwicklung kreativer Fähigkeiten von Kindern, zur Entspannung und Entlastung von Arbeit und als Medium, um mit Kindern zu kommunizieren. In dieser Zeit wird die Rolle des Spiels in der allgemeinen Erziehung von Kindern stark beachtet. Es handelte sich noch nicht spezifisch um Fremdsprachenunterricht oder Spielandragogik.

Die positive Einschätzung des Spiels für die Entwicklung des Menschen und die unterrichtliche Verwendung geht in der zweiten Hälfte des 19. Jahrhunderts im Zug des Herbartianismus und des aufkommenden Frontalunterrichts wieder verloren und wird erst mit der Reformpädagogik des 20. Jahrhunderts wieder ins Zentrum pädagogischer Betrachtung zurückgebracht (vgl. Kube 1977, S. 27f.; Klippel 1980a, S. 15).

Die Aufmerksamkeit der Wissenschaft gilt allerdings traditionell vorwiegend dem kindlichen Spiel (vgl. Hansen und Wendt 1990, S. 15). Der Spielandragogik fehlen noch gesicherte Grundlagen. Allerdings kann man überall eine Übertragung auf Erwachsene finden (vgl. ebenda, S. 17). Kube (1977, S. 8) hebt hervor, dass die Entwicklung einer entsprechenden Spieldidaktik erst am Anfang steht.

Die Forschungsentwicklung des fremdsprachendidaktischen Aspektes vom Spiel wurde wegen des fächerübergreifenden Charakters des Spiels und auch des Verhältnisses zwischen allgemeiner Didaktik und Fremdsprachendidaktik, die nicht als besonders eng bezeichnet werden kann, verhindert (vgl. Klippel 1980a, S. 51). Am Ende der 70er und Anfang der 80er Jahre sind einige wichtige AutorenInnen mit ihren Werken zum Spieleinsatz im Fremdsprachenunterricht zu nennen: Klippel 1980b, 1980a; Steinhilber 1979; Kleppin 1980. Allerdings wird das Thema danach nur sporadisch behandelt.

Das bestätigt Hoppe (1983, S. 286) durch seine Untersuchung der Zeitschrift "Praxis des neusprachlichen Unterrichts", in der er in den Jahrgängen 1953 bis 1978 nur drei kleine Beiträge zur Thematik Spiel bzw. darstellendem Spiel im Fremdsprachenunterricht fand, also insgesamt 20 Seiten in den genannten Zeitschriften, in denen mehr als 5000 Seiten fachdidaktische Themen behandelt werden. Eine zunehmende Aufgeschlossenheit für Fragen der Spielverwendung im Fremdsprachenunterricht fand Kube aber im Jahr 1979, wo sich die erste Ausgabe der genannten Zeitschrift dem Thema „Lernspiele für die Praxis“ widmete. Es gab etwa zur gleichen Zeit auch erste Ansätze von systematischer Behandlung 
und Begründung unterrichtlichen Spielens im Fremdsprachenunterricht: Kleppin (1980) und Steinhilber (1979).

Der Überblick der spieltheoretischen Forschung zeigt, dass weder alle Funktionen noch alle Erscheinungsformen des Spiels vollständig bekannt und untersucht sind. Im Hinblick auf die Eigenschaften und Wirkungen von Spielen ist noch vieles ungeklärt (vgl. Klippel 1980a, S. 14). Wenn man sich den Stand der Diskussion und die vorhandenen Materialien ansieht, bekommt man den Eindruck, dass Spiele eine allgemein anerkannte Stellung in der Fremdsprachendidaktik gefunden hätten. Allerdings widerspricht die Praxis der „vorgeblichen Spielfreudigkeit“. Spiele sind trotz der umfangreichen dafür sprechenden Theorie eine "Randerscheinung“ des Fremdsprachenunterrichts (vgl. Kleppin 1980, S. 1; Klippel 1998, S. 4).

Die von Klippel (1998) zusammengestellte Auswahlbibliografie zu Spielen im Fremdsprachenunterricht zeigt deutlich, dass die Spielpädagogik und die Spieldidaktik im Fremdsprachenunterricht in den 1970er Jahren ihre goldene Zeit erlebten. Viele Werke zum Thema Spiel, zur Theorie und Geschichte der Spielforschung, sind in den siebziger Jahren erschienen. Umfangreiche Literatur zur Spielvorschlägen und Spielmaterialien ist auch in den achtziger Jahren zu finden. Ab 1990 wurden hauptsächlich Spielesammlungen und Spielmaterialien herausgegeben, allerdings keinerlei weiterführende theoretische oder empirische Forschungen (vgl. Koenig 2003, S. 9; Klippel 1998, S. 13). Spiele werden in den 70er und 80er Jahren so stark erforscht, dass ab den neunziger Jahren Spielelemente generell Eingang in Lehrwerke und Übungsmaterialien (in Deutschland) finden (vgl. Niewalda 2014, S. 241; Klippel 1998, S. 4).

Heutzutage, 50 Jahre nach der goldenen spieldidaktischen Zeit des 20. Jahrhunderts, bleibt der Forschungsschwerpunkt des Sprachlernspiels immer noch in der theoretischen Welt von Spielesammlungen und -materialien. Es wird angenommen, dass Spiele motivierend sind, Angst abbauen können, und positive Wirkungen im Unterricht haben. Es gibt Themenhefte der einschlägigen Zeitschriften, die den Spielen und spielerischen Übungen gewidmet sind. Aber ob diese umfangreiche Literatur zum Thema Spiel im Fremdsprachenunterricht überhaupt für den Spieleinsatz in der Praxis führt, ist unbekannt (vgl. Koenig 2003, S. 9).

Dass es Bedarf an weiterer empirischer Forschung zum Spieleinsatz im Fremdsprachenunterricht besteht, bestätigen viele Autoren (vgl. Hansen und Wendt 1990, S. 41; Koenig 2003, S. 9; Bohn und Schreiter 2001, S. 420; Mátyás 2009, S. 114). Dazu erörtert Jentges (2007, S. 36) beispielsweise, dass sich die bis 2007 verfassten wissenschaftlichen Arbeiten hauptsächlich mit dem Sprachlernspiel im nicht-deutschen, also Fremdsprachen-Unterricht im schulischen 
Kontext beschäftigen. Jentges (2007, S. 40-56) analysiert die Untersuchungen zur Wirkung der Motivationssteigerung von Sprachlernspielen und meint, die allgemein verbreitete Annahme, dass Sprachlernspiele im Fremdsprachenunterricht motivierende Funktion haben, kann nicht belegt werden und bedarf weiterer Untersuchungen. Den Untersuchungen zur Effizienz des Erreichens von fremdsprachlichen Lernzielen sei es auch nicht überzeugend gelungen, die Effizienz von Sprachlernspielen als Lernform im Fremdsprachenunterricht nachzuweisen.

Klippel (2010, S. 189) erwähnte folgenden Gründe für die entzogene empirische Validierung des Spiels:

1. die Individuell unterschiedlichen Reaktionen von Lernenden auf Spiele.

2. Fremdsprachenlehrkräfte gründen ihre Verwendung von Sprachlernspielen auf ihre individuelle Haltung gegenüber spielerischen Aktivitäten.

3. Eine empirische Spielforschung müsste erhebliche Hürden überwinden, denn die Vielzahl der Faktoren, die Spielfreude und Spielerfolg beeinflussen, lässt ein experimentelles Forschungsdesign wenig geeignet erscheinen.

Zum zukünftigen Forschungsbedarf schreibt sie (ebenda, S. 189): „Es ist die Aufgabe der fremdsprachendidaktischen Forschung zu untersuchen, warum und in welcher Hinsicht bestimmte Spiele oder activities gute Lernsituationen darstellen."

Im Folgenden werden einige empirische Untersuchungen zum Sprachlernspiel im Fremdsprachenunterricht vorgestellt, um einen Überblick über die bisherige Praxis der Spieldidaktik im Fremdsprachenunterricht zu geben.

\subsubsection{Empirische Forschungen zum Einsatz der Sprachlernspiele im DaF-Unterricht}

In diesem Teil werden einige empirische Forschungen zu Sprachlernspielen im Fremdsprachenunterricht vorgestellt. Forschungen aus europäischen und asiatischen Räumen werden hier unter die Lupe genommen, um den Einsatz vom Spiel in den verschiedenen Kulturkreisen näher zu betrachten.

\subsubsection{Empirische Forschungen zum Einsatz der Sprachlernspiele im europäischen Raum}

Seit der kommunikativen Wende gewinnt das Spiel einen festen Platz im Fremdsprachenunterricht (vgl. Kleppin 2017, S. 325). Steinhilber (1979/1986) und Kleppin (1980) gehören zu den ersten, die Sprachlernspiele im Rahmen von Fremdsprachenunterricht in Deutschland untersucht haben. 
Die Forschung von Steinhilber (1979/1986) fokussiert sich auf die Wirkung des Spieleinsatzes auf Leistung und Motivation im schulischen Lateinunterricht. Das Experiment, das mit Experimental- und Kontrollgruppen durchgeführt wird, hat insgesamt 14 Tage gedauert. Der Lernerfolg wurde durch Vortest-Nachtest-Behaltenstest definiert. Zwischen dem Vortest und dem Nachtest wurde die Experimentalgruppe mit Spieleinsatz, und die Kontrollgruppe konventionell, also ohne Spiele, unterrichtet. Zwischen den Nachtests und den Behaltenstests wird in beiden Gruppen konventionell gelehrt, um den Lernerfolg zu überprüfen. Die Beurteilung der Motivationsfunktion des Spiels wird durch Befragung erfasst. Zusammenfassend lässt sich sagen, dass die Lernenden der Experimentalklasse mit Spieleinsatz mehr gelernt haben als die der Parallel-Klasse ohne Spiele. Eine erhöhte Motivation der Schüler kann man in der Spiele-Klasse beobachten. Allerdings treten die Einstellungsänderungen nicht so deutlich hervor wie der Lernerfolg. Die eingesetzten Unterrichtsspiele können zu besseren Lernund Behaltensergebnissen führen, auf die Einstellung der Schüler zum Fach üben sie aber keinen nachhaltigen Einfluss aus (vgl. Steinhilber 1986, S. 135).

Die Hauptforschungsziele von Kleppin (1980) sind die Beschreibungen des Verhaltens sowohl von Lernenden als auch von Lehrenden im Sprachlernspiel, und zwar mit einem selbstentwickelten Untersuchungsinstrumentarium. Mit Hilfe des Instrumentariums sollen die Interaktionsverläufe in Spielen und die subjektive Einstellung gegenüber den Spielen dargestellt und beschrieben werden. Das Instrumentarium besteht aus einer Reihe von Befragungen und Unterrichtsprotokollen zum Interaktions- und Zeitverlauf.

Die Untersuchung wurde im Französisch-Unterricht im Hochschulbereich durchgeführt und versuchte damit aufzuzeigen, dass auch für Erwachsene das Sprachlernspiel durchaus effektiv sein kann. Um die Effizienz von Spielen zu messen, werden zwei verschiedene Gruppen unter kontrollierten Unterrichtsbedingungen als Probanden genommen. Der Lernerfolg wird nicht durch einen Test, sondern durch die subjektiven Beurteilungen der Lernenden bestimmt, weil ein Abschlusstest der Forscherin ,aufgrund der vielen nicht kontrollierbaren äußeren Variablen (z. B. die psychische Verfassung der Probanden)“ (ebenda, S. 62) unmöglich zu konstruieren war, und der „Scheinobjektivität“ vieler Untersuchungen ihrer Meinung nach die Problematik verschleiern kann (vgl. ebenda, S. 61-63). Die Untersuchung fand in drei Kursen statt. Jedes durchgeführte Sprachlernspiel wurde mit einem Video aufgenommen. Mehrere Lehrende nahmen an der Forschung teil. Am Ende wurden insgesamt 11 Spiele in die Bewertung einbezogen (vgl. ebenda, S. 152-362).

Die Forschung von Kleppin (1980) zeigt: Obwohl man bei der Durchführung des Spiels mit vielen Schwierigkeiten und Problemen konfrontiert sein kann, sollen und 
können Sprachlernspiele ihren sinnvollen Platz in der Fremdsprachenmethodik haben, wenn der Einsatz bewusst erfolgt. Sprachlernspiele bedürfen einer genauen Planung durch die Lehrperson, die dafür bereit ist, Signale aus der Lerngruppe aufzunehmen, zu verarbeiten, und zu versuchen, Gründe für Spielhemmungen, Unlust am Spiel, Probleme mit Korrekturen usw. herauszufinden und eigenes Verhalten zu verändern (vgl. ebenda, S. 466).

Wie im Kapitel 3.3.1 diskutiert, gibt es nur begrenzte empirische Forschungen, die Wirkungen von Sprachlernspielen systematisch belegen können. Jentges (2007) versuchte mit ihrer Forschung zur Effektivität des Sprachlernspiels auf Wortschatzerwerb dieses Forschungsdefizit zu verbessern. Sie führt eine systematische empirische Forschung mit zwei unabhängigen Gruppen anhand des Lateinischen Quadrates durch. Die Datenerhebungsmethode besteht aus vier Prüfungen, die über einen dreimonatigen Zeitraum stattfinden. Die Probanden sind ausländische Studierende an der Phillips-Universität Marburg. Um die Untersuchung durchzuführen, wird eine zweiwöchige Lehrveranstaltung mit dem Titel „Spielend Deutsch lernen“8 in drei Semestern kostenlos angeboten. Insgesamt haben 105 Personen an der Lehrveranstaltung teilgenommen. Die Kursteilnehmenden kommen aus 34 Ländern, wobei nur 9 Personen ${ }^{9}$ aus dem ostasiatischen Raum stammen. Die Mehrheit davon kommt aus englischsprachigen Ländern.

In dieser Forschung werden Methoden in beiden Gruppen anschaulich gemacht, sodass man weiß, dass das Experiment in einem methodisch vergleichbaren Kontext stattfindet. Die Ergebnisse zeigen, dass der Unterricht mit Sprachlernspielen kurz- und mittelfristig effizienter als der Unterricht ohne Spiele ist. Auf das langfristige Behalten haben die zwei Vermittlungsmethoden keinen Unterschied (vgl. ebenda, S. 110f.). Insgesamt gesehen können Sprachlernspiele als effiziente Unterrichtsmethode im Vergleich mit anderen betrachtet werden und sollten einen festen Platz im Fremdsprachenunterricht haben. Gleichzeitig wird auch betont, dass ein individueller Akzeptanzunterschied von

8 Bei der spielerischen Vermittlungsmethode in der genannten Forschung soll betont werden, dass Jentges Sprachlernspiel im engeren Sinn versteht als in der vorliegenden Arbeit. Sie betont, dass Sprachlernspiele als „echte Spiele“ wahrgenommen werden sollen. Sie sieht z. B. Kreutzworträstel nicht als Spachlernspiel, sondern nur als spielerische Übung an (vgl. Jentges 2007, S. 51). In der vorliegenden Arbeit wird allerdings die spielerische Übung als Unterbegriff vom Sprachlernspiel verstanden, wobei Kreuzworträtsel als Sprachlernspiel betrachtet wird.

9 Darunter sind 5 aus Japan, 2 aus Taiwan und 2 aus Südkorea (vgl. Jentges 2007, S. 67). 
Sprachlernspielen in der Forschung deutlich wird. In der Zukunft sollen ihrer Meinung nach noch weitere Forschungen zur Akzeptanz von Sprachlernspielen bei Lernenden mit verschiedenen persönlichen, kulturellen und sozialen Merkmalen durchgeführt werden (vgl. ebenda, S. 123).

Während die Forschungen von Steinhilber (1979/1986), Kleppin (1980) und Jentges (2007) einen Blick auf den Spieleinsatz im Fremdsprachenunterricht in Deutschland bieten, untersuchen Mátyás (2009) und Kacjan (2008) die Rolle von Sprachlernspielen im DaF-Unterricht in Finnland, Ungarn und Slowenien.

Das Forschungsprojekt von Mátyás (2009) wurde als Dissertationsarbeit an der Universität Jyväskylä, Finnland vorgelegt. Ziel der Untersuchung ist, die Spielpraxis im Fremdsprachenunterricht der gymnasialen Oberstufe in Finnland und Ungarn zu beschreiben und zu vergleichen. Dabei werden die Meinungen der Lehrenden zum Spieleinsatz im DaF-Unterricht berücksichtigt. Die Forschungsergebnisse zeigen, dass der Spieleinsatz im finnischen und ungarischen DaF-Kontext wie in früheren Forschungsarbeiten (vgl. Koenig 2003, S. 10; Klippel 1998, S. 4) als Randerscheinung angesehen wird, obwohl die Sprachlernspiele nach der subjektiven Einschätzung der Lehrkräfte in beiden Unterrichtskontexten als eine gleichberechtigte Unterrichtstechnik betrachtet werden. Als Gründe des mangelnden Spieleinsatzes wurden von den Befragten Zeitmangel im Unterricht sowie schwierige Zugänglichkeit der Sprachlernspiele genannt (vgl. Mátyás 2010, S. 392f.).

Signifikanter Unterschied zwischen dem finnischen und dem ungarischen DaF-Unterricht konnte beim Einsatz verschiedener Spieltypen bemerkt werden. Während im finnischen Kontext kooperative Sprachlernspiele bevorzugt werden, sind die kompetitiven Sprachlernspiele bei ungarischen Lehrkräften ein beliebter Spieltyp. Die Forscherin begründet, dass es im finnischen Fremdsprachenunterricht eine lange Tradition kooperativer Lernformen gibt. Zusammenfassend lässt sich sagen, dass die Unterrichtskultur und -tradition eines Landes, die Leitlinien der Lehrpläne und der didaktische Hintergrund der Lehrkräfte die Unterrichtspraxis stark beeinflussen können (vgl. ebenda, S. 393).

Die Kacjan-Forschung (2008) findet im schulischen Kontext in Slowenien statt und hat auch die Spielwirkung auf den Wortschatzerwerb zum Ziel. Wegen der geringen Probandenzahl (6 Schüler) (vgl. ebenda, S. 103) wird hier darauf verzichtet, eine weitere Darstellung dieser Forschung zu liefern. Zum nächsten wenden wir den Blick auf die Spielpraxis in Asien. Da es nur sehr begrenzte Literatur zum Spieleinsatz im Fremdsprachenunterricht in asiatischem Raum gibt, werden im Folgenden die empirischen Forschungen zum Spieleinsatz im DaFUnterricht in Japan und in China berücksichtigt. 


\subsubsection{Empirische Forschungen zum Einsatz der Sprachlernspiele in Japan}

Laut Niewalda (2014, S. 241) gibt es nur wenige Studien in Japan, die Sprachlernspiele im Fremdsprachenunterricht untersuchen. Es gibt eine große Distanz vom Forschungstand in Japan mit Vergleich zu dem im deutschsprachigen Raum. Um diese Forschungslücke zu füllen, hat Niewalda zwei empirische Forschungen jeweils aus Lehrer- und Lernerperspektive durchgeführt.

In der Forschung zum Spieleinsatz aus Sicht der Lernenden versucht Niewalda (2014) herauszufinden, ob die Lernenden Sprachlernspiele als effektive Lehrmethode einschätzen und wenn ja, inwiefern. Die Forschung ist als Aktionsforschungsprojekt zu verstehen und besteht aus drei Teilen. Zum ersten wurden zwei Spiele im Unterricht eingesetzt, dann diskutierten die Lernenden in Kleingruppen über ihre Spielerfahrung. Der dritte Bestandteil ist das Lehrtagebuch der Forscherin als Ergänzung zu den Ergebnissen der Diskussionen.

Die Untersuchung fand im Juli 2013 an der Universität Matsuyama satt. 40 Studierende aus unterschiedlichen Fachrichtungen, die Deutsch als zweite Wahlpflichtfremdsprache lernen, bildeten die Probanden. Die Probanden haben sehr wenige Erfahrungen mit Sprachlernspielen und sind Deutsch-Anfänger. Insgesamt werden zwei Spiele im Unterricht eingesetzt und evaluiert. Gleich nach der Durchführung vom Spiel wurde in Kleingruppen darüber diskutiert. Alle Diskussionen wurden mit einem Aufnahmegerät aufgezeichnet und transkribiert (vgl. ebenda, S. 247-250).

Zusammenfassend lässt sich sagen, dass der Großteil der Studierenden Sprachlernspiele als positiv einschätzen und als ernstzunehmende Arbeitsform im DaF-Unterricht wahrnehmen. Die Lernenden sind sich bewusst, dass Sprachlernspiele nicht nur die strukturelle sprachliche Fähigkeit verbessern, sondern auch kommunikative sowie soziale Techniken vermitteln können. Die Effektivität von Sprachlernspielen im DaF-Unterricht wird von den Lernenden bestätigt (vgl. Niewalda 2014, S. 256).

Auf der organisatorischen Ebene wurden einige problematische Aspekte von Studierenden genannt, dass es zum Beispiel zeitlich nicht ausreichend war, ein Spiel zu Ende zu spielen, oder dass die Regeln nicht für alle verständlich waren. Außerdem fühlten manche sich überfordert, weil das Spiel ihrer sprachlichen Kompetenz nicht ganz entsprach (vgl. ebenda, S. 255f.).

Die zweite Forschung von Niewalda (2015) liefert einen ersten Einblick zum Einsatz von Sprachlernspielen an japanischen Universitäten aus Lehrer-Perspektive (vgl. Niewalda 2015, S. 127). Diese empirische Forschung zeigt eine 
ausführliche Darstellung über die Stellungnahmen der DaF-Lehrenden an japanischen Universitäten über den Einsatz von Sprachlernspielen.

Das Forschungsinstrument bestand aus Fragebögen. Diese wurden 2013 in drei Fachtagungen sowie Konferenzen in Japan ausgegeben. Von 210 ausgegebenen Fragebögen wurden 42 zurückgeschickt. Um die 20 \% Rücklaufquote zu erhöhen, wurde der Fragebogen 2014 noch einmal online angeboten. 25 zurückgeschickte Fragebögen der Onlinebefragung wurden in die Auswertung einbezogen. Außerdem wurden Interviews mit sechs Lehrenden durchgeführt (vgl. ebenda, S. 129-131).

Unter den 67 Befragungsteilnehmenden waren 34 japanische Lehrende und 26 deutsche Lehrende. 46 davon hatten mehr als 10 Jahre Unterrichtserfahrung, nur 4 unterrichteten weniger als 3 Jahre. $23 \%$ der befragten Lehrenden hatten einmal an einer Fortbildung zum Thema Sprachlernspiel teilgenommen und $65 \%$ hatten Interesse an einer solchen Veranstaltung gehabt (vgl. ebenda, S. 132).

Zusammenfassend lässt sich sagen, dass die befragten Lehrenden, sowohl deutsche als auch japanische, eine positive Stellung gegenüber Sprachlernspielen hatten. Durch die relativ niedrig Rücklaufquote der Fragenbogen kann man annehmen, dass die Problematik „Sprachlernspiel“ für die meisten japanischen Lehrenden nicht interessant oder attraktiv genug war, da es zwischen der Anzahl der deutschen (26) und die der japanischen (34) Beteiligten keinen großen Unterschied gab, obwohl die Befragungen in Japan durchgeführt wurden.

Die Mehrheit der Befragten verwendeten Sprachlernspiele nicht regelmäßig und setzten sie in einem Semester nur ein- bis dreimal ein (vgl. ebenda, S. 135). Manche Lehrenden meinten, dass die Lernenden Zeit für die Gewöhnung haben sollten (vgl. Niewalda 2015, S. 146). Viele Lehrende waren der Meinung, dass Sprachlernspiele aufwendige Vorbereitungen benötigen und bei der Durchführung zeitintensiv sein können (vgl. ebenda, S. 148). Die japanischen Befragten wiesen noch darauf hin, dass sie als Lehrende keine Erfahrungen mit Sprachlernspiele in eigenen Lernprozessen gemacht haben, und wussten deswegen nicht, wie man das macht (vgl. ebenda, S. 150f.). Darüber hinaus sollen die Rahmenbedingungen wie Gruppenstärke, Raumbedingungen und das Image der Universität eine entscheidende Rolle im Spieleinsatz spielen. Ein japanischer Lehrer schrieb, dass Sprachlernspiele an bestimmten Universitäten nicht erwünscht sind, sogar als Tabu betrachtet werden (vgl. ebenda, S. 151f.).

Infolgedessen kann man sagen, dass Sprachlernspiele im DaF-Unterricht in Japan auch nur eine Randerscheinung im Unterricht sind, obwohl der Einsatz von Sprachlernspielen von den Lernenden befürwortet wird. Die Wirkung vom Spieleinsatz wird in der Praxis von den Lehrenden bestätigt. Allerdings finden 
die meisten Befragten, dass es zeitintensiv ist, die Spiele vorzubereiten und durchzuführen.

\subsubsection{Empirische Forschungen zum Einsatz der Sprachlernspiele in China}

Eine systematische Forschung zum Sprachlernspiel im Fremdsprachenunterricht in China ist nicht zu finden. Zwei Dissertationsarbeiten zu den Unterrichtsmethoden liefern einen begrenzten Blick zum Spieleinsatz im chinesischen DaF-Unterricht für das Vokabellernen.

Beide Dissertationsarbeiten versuchen, die verschiedenen Lernstrategien für Vokabellernen im DaF-Unterricht in China zu vergleichen. Während Yeh (2014) die Akzeptanz von verschiedenen Methoden bei chinesischen Lernenden untersucht, spielt bei der Untersuchung von Yang (2017) die Effektivität von verschiedenen Methoden die Hauptrolle.

Die Forschung von Yeh (2014) befasst sich mit der Akzeptanz von fünf verschiedenen Wortschatzlernmethoden bei chinesischen Lernenden. In der Forschung werden insgesamt 754 Germanistik-Studierende befragt (vgl. ebenda, S. 116), um ihre Meinung gegenüber den verschiedenen Methoden zu sammeln. Dabei werden die linguistisch-kognitive Methode, die situativ-imitative Methode, Sprachlernspiele, multimediale und internetgestützte Methoden und die Vermittlung von Wortschatzlernstrategien und -techniken als Methode berücksichtigt (vgl. ebenda, S. 80). Die Forschung zeigt, dass die Befragten nur sehr wenig Erfahrung mit Sprachlernspielen haben (vgl. ebenda, S. 119). Die Methode wird von der Mehrheit der Befragten als positiv bewertet (vgl. ebenda, S. 149). Allerdings zeigen Sprachlernspiele im Vergleich mit anderen Methoden relativ niedrigere Akzeptanz (vgl. ebenda, S. 199). Die Akzeptanz der linguistisch-kognitiven Methode liegt am höchsten (vgl. ebenda, S. 194).

Yang (2017) hat vier Methoden verglichen. Zwei davon sind in China weitverbreitet und häufig eingesetzt, nämlich Auswendiglernen und Vokabellernen im Wortfeld. Die anderen zwei sind in China selten eingesetzt, nämlich das „Memory“-Spiel und digitale Lernprogramme (vgl. ebenda, S. 147). Die empirische Forschung wird nach dem Forschungsverfahren des Lateinischen Quadrats durchgeführt, dauert insgesamt sechs Wochen bzw. acht Unterrichtsstunden an einer chinesischen Universität. Insgesamt haben 45 chinesische Bachelor-Studierende im Fach Germanistik an der Forschung teilgenommen. Als Datenerhebungsmethoden werden Tests und Befragungen eingesetzt, um Lernfortschritte, Lernerbiographie und Meinungen gegenüber eingesetzten Methoden von den Lernenden zu sammeln. Die Forschungsergebnisse zeigen, dass Sprachlernspiele 
im Vergleich zu den traditionellen Methoden besser von den Studierenden bewertet werden, weil sie die Atmosphäre im Unterricht verbessern und interaktionsanregender sind (vgl. ebenda, S. 190). Auch die Testergebnisse bestätigen die bessere Wirkung vom Sprachlernspiel als Auswendiglernen und WortfeldMethode (vgl. ebenda, S. 208). Außerdem wird herausgefunden, dass die Lernenden kaum Erfahrung mit spielerischer Übungsform haben (vgl. ebenda, S. 188).

Die zwei Forschungen bestätigen, dass der Einsatz von Sprachlernspielen bis jetzt eine seltene Methode im DaF-Unterricht in China ist. Anders als in Deutschland, gewinnt der Spieleinsatz im Fremdsprachenunterricht erst in den letzten Jahren Aufmerksamkeit in China. Es gibt kaum von Chinesen konzipierte Lehrwerke mit Sprachlernspielen oder Spielesammlungen für Fremdsprachenunterricht. Erst in der jüngeren Zeit gewinnt das Spiel Raum in chinesischen DaF-Lehrwerken. Liangs (2018) Untersuchung zur spielerischen Übungen in den Lehrwerk-Reihen "Studienweg Deutsch“ gibt den ersten Überblick über Spiele in chinesischen DaF-Lehrwerken. Es wird herausgefunden, dass spielerische Übungen in genannten Lehrwerken etwa $7 \%$ bis $10 \%$ der Gesamtübungen einnehmen, wobei Rollenspiele und Brainstorm auch spielerischen Übungen zugeordnet werden (vgl. ebenda, S. 102). Nach eigener Unterrichtserfahrung bemerkt Liang (ebenda, S. 103), dass spielerische Übungen sehr zeitaufwendig sind. Viele Lehrende behandeln die spielerischen Übungen nicht, weil sie keine Zeit dafür haben. Oder sie führen solche Übungen mit traditioneller lehrerzentrierter Methode durch. Außerdem wirken sich die mangelnden Variationen nach bestimmter Zeit nur langweilig für die Lernenden aus.

Zusammenfassend kann man feststellen, dass Sprachlernspiele in China genau so wenig wie in Japan behandelt werden. Es gibt bisher keine systematische Forschung zum Einsatz von Sprachlernspielen. Diese werden auch in der Unterrichtspraxis wenig verwendet.

\subsubsection{Schlussfolgerung zu den empirischen Forschungen}

Jede der vorgestellten Forschungen besitzt ihre eigene Perspektive zum Thema Sprachlernspiel. Manche Forschungen betrachten hauptsächlich subjektive Meinungen (vgl. Kleppin 1980; Mátyás 2009; Niewalda 2014, 2015; Liang 2018). Andere Forschungen versuchen die Wirkung des Spieleinsatzes mit Hilfe von Tests zu bestätigen (vgl. Steinhilber 1986; Jentges 2007; Kacjan 2008; Yang 2017).

Durch die Studien in den letzten 10 Jahren kann festgestellt werden, dass die Lern- und Lehrtradition den Einsatz von Sprachlernspielen im Fremdsprachenunterricht stark beeinflussen können. Aufgrund verschiedener Lerntraditionen 
können verschiedene Spieltypen vorkommen (vgl. Mátyás 2009). Die Problematik vom Sprachlernspiel ist im Fremdsprachunterricht in China, genau wie in Japan, sehr wenig erforscht. Der Einsatz von Sprachlernspielen kann von den betroffenen regionalen Rahmenbedingungen be- oder sogar verhindert werden. Deswegen ist es sinnvoll, eine Organisationsmethode für spielerische Unterrichtsmethoden zu entwickeln, die in die Lehr- und Lerntradition und die spezifischen Rahmenbedingung in China integriert werden kann. Die „westliche“ Methode soll modifiziert werden, um sich dem chinesischen Unterricht anzupassen und dort Raum zu erhalten. Auch Jentges (2007, S. 123) spricht über weiteren Forschungsbedarf zum Spieleinsatz in anderem Lernkontext.

Obwohl die Forschungen einen wichtigen Beitrag zum Sprachlernspiel geleistet haben, zeigen sie einige gemeinsame methodische Schwächen:

1. Die Dauer der empirischen Forschungen ist m. E. ungenügend. Die Experimentierzeit der Wirkungsforschungen mit Tests dauert von 14 Tage (vgl. Steinhilber 1986) bis drei Monate (vgl. Jentges 2007). Es fehlen Langzeitergebnisse, die die Wirkungen von Sprachlernspielen auf Lernfortschritte bestätigen können.

2. Die eingesetzten Spiele werden meistens nicht als normaler Unterrichtsbestandteil behandelt, sondern als Forschungsmethode in Sonderstunden oder Sonderkursen eingesetzt. Bei der Kleppin- und Niewalda-Forschung beispielsweise werden die eingesetzten Spiele nicht in die Unterrichtseinheit integriert. In der Niewalda-Forschung (2014) finden die Diskussionen gleich nach dem Spiel in derselben Stunde statt. So ist der Unterricht wegen des Spieleinsatzes und der Befragung bzw. Diskussion nicht vollständig. Bei der Forschung von Jentges (2007) nehmen die Probanden in einer spielspezifischen Lehrveranstaltung teil.

3. Die Anzahl der eingesetzten Spiele ist begrenzt. In der Forschung von Kleppin (1980) werden 11 Spiele analysiert, während die ganz Forschung 2 Semester dauert. Die Niewalda-Forschung hat nur 2 Spiele analysiert. Das heißt, dass die Sprachlernspiele auch in den meisten genannten zum Spieleinsatz nicht als reguläre Unterrichtsmethode eingesetzt werden.

4. Bei der Mehrheit der Forschungen zu den Wirkungen der Sprachlernspiele auf den Lernfortschritt handelt es sich um Vokabellernen (vgl. Jentges 2007; Kacjan 2008; Yang 2017), aber nicht um die ganzheitliche Sprachfähigkeit. Sprachlernspiele sind allerdings in allen Unterrichtsphasen zur verschiedenen Lernzielen einsetzbar (vgl. Kleppin 2017, S. 325).

5. Bei der Mehrheit der Wirkungsforschungen bezüglich Lernfortschritt werden die Unterrichtsverläufe der Kontrollgruppe nicht genau dargestellt (vgl. 
Steinhilber 1986; Kacjan 2008). Es kann nicht festgestellt werden, ob der bessere Lernfortschritt von der spielerischen Methode oder nur von einer längeren Übungszeit und mehr Übungsmöglichkeiten verursacht wird.

6. Es gibt keinen Vergleich auf der zeitlichen Ebene. Es wird nicht nachgewiesen, ob Sprachlernspiele mehr Zeit im Unterricht in Anspruch nehmen als vergleichbare traditionelle Übungen.

7. Bis heute ist der Einsatz von Sprachlernspielen im Unterrichtsalltag an chinesischen Universitäten unsystematisch erforscht. Die interkulturelle Barriere wird von Koenig (2003, S. 16) als Schwierigkeit des Spieleinsatzes angesehen. Der sozialkulturelle Hintergrund, die Rahmenbedingungen vor Ort, die Lern- und Lehrtradition sowie deren Einfluss auf Spieleinsatz werden unzureichend betrachtet.

\subsection{Zwischenfazit}

Es kristallisiert sich heraus, dass das Sprachlernspiel eine wichtige Unterrichtsmethode sein soll. Die im Kapitel 3.3 diskutierten Effekte zeigen, dass Sprachlernspiele einen speziellen Beitrag für DaF-Unterricht in China leisten können:

Sprachlernspiele können die Lernenden motivieren, weil sie lustanregend sind und eine angstfreie Unterrichtsatmosphäre bilden können. Durch Sprachlernspiele kann die Gruppenzugehörigkeit gefördert werden, sodass man sich in der Gruppe sicher fühlt. Da Angst, Peinlichkeit und Stress zur Schweigsamkeit im Unterricht führen (vgl. Smith und King 2018, 325), können eine verbesserte Unterrichtsatmosphäre und starke Gruppenzugehörigkeit die Kommunikationsbereitschaft (WTC) fördern.

Außerdem werden Sprachlernspiele meistens in der Form von Gruppen- oder Partnerarbeit durchgeführt, was für die Erhöhung von WTC auch von besonderer Bedeutung ist (siehe Kapitel 2.2.4). Diese beide Sozialformen sind eben für guten Großgruppenunterricht vorrangig (siehe Kapitel 2.2.3.2).

Sprachlernspiele bieten Chancen für kontextuelles Lernen mit allen Sinnen an, erhöhen Aufmerksamkeit und Sprechzeit und fördern autonomes Lernen (siehe Kapitel 3.3.2.3). Mit der erhöhten Motivation und Interaktion im Unterricht ist zu erwarten, dass Sprachlernspiele die sprachliche Fähigkeit der Lernenden begünstigen können.

Zusammenfassend lässt sich sagen, dass Sprachlernspiele eine ideale Unterrichtsmethode gegen die Schweigsamkeit und den Interaktionsmangel der chinesischen Fremdsprachenlernenden sein können. Allerdings wird die Wirkung 
von Sprachlernspielen im DaF-Unterricht noch nicht systematisch erforscht, sowie deren Einsetzbarkeit und Akzeptanz im chinesischen Kontext.

In der vorliegenden Untersuchung wird versucht, den heutigen Untersuchungsstand zum Einsatz der Sprachlernspiele im Fremdsprachenunterricht zu ergänzen. Die Effizienz von Sprachlernspielen im chinesischen DaF-Unterricht wird auf Leistungs- und Motivationsebenen überprüft. Außerdem wird die praxisorientierte organisatorische Methode des Einsatzes der Sprachlernspiele in China mit einbezogen. Dabei wird beispielsweise herausgefunden, ob Sprachlernspiele zeitlich genau so effizient wie traditionelle Übungen organisiert werden können, und ob man traditionelle Übungen als Sprachlernspiele adaptieren kann. Das letzte Ziel ist für den DaF-Unterricht in China von herausragender Bedeutung, weil es kaum Spielesammlungen für den Fremdsprachenunterricht in China gibt und man manchmal mit einem bestimmten Lehrwerk arbeiten muss, das kein spielerisches Element enthält. Der Blick richtet sich eben auf die regionale Besonderheit und deren Einfluss auf den Spieleinsatz, weil die Rahmenbedingungen in China anders als die in Europa sind (siehe Kapitel 2.2).

Darüber hinaus sollen die Sprachlernspiele als fester Bestandteil eines einheitlichen Unterrichts integriert werden, sodass sie als eine reguläre Methode des Unterrichts von Studierenden wahrgenommen werden können. Außerdem kann die ganzheitliche Wirkung von Sprachlernspielen aufs Erlernen einer Fremdsprache dadurch überprüft werden, was bisher noch nicht systematisch behandelt wird. Im Folgenden wird die Herangehensweise der empirischen Untersuchung veranschaulicht. 



\section{Die empirische Untersuchung: Hintergrund und Herangehensweise}

Den Forschungen zu Sprachlernspielen im Fremdsprachenunterricht ist es bis heute nicht gelungen, genügend empirisch belegte Beweise zur Wirkung der Sprachlernspiele im Fremdsprachenunterricht zu liefern. Einerseits können die empirischen Forschungen keinen hinreichenden Nachweis für die Effizienz des Sprachlernspiels gegenüber dem Unterricht ohne Sprachlernspiel belegen. Andererseits wurde noch keine empirische Untersuchung durchgeführt, um die Fragen zu beantworten: Kann eine solche interaktive/kommunikative Methode im Fremdsprachenunterricht effizient sein in Ländern, wo der Fremdsprachenunterricht noch stark von einem lehrerzentrierten Unterricht und der Grammatik-Übersetzungsmethode geprägt ist?

Die vorliegende Forschung versucht deswegen die Effizienz der Sprachlernspiele in einem anderen kulturellen Kontext, d. h. im DaF-Unterricht an chinesischen Universitäten zu überprüfen. Es wird davon ausgegangen, dass Sprachlernspiele multifunktionale Hilfsmittel vom Fremdsprachenunterricht für eine Zielgruppe aus allen Lerntraditionen sein können, weil spielendes Lernen in der Natur des Menschen liegt. Wenn man die Sprachlernspiele vorsichtig adaptiert und den Unterricht sorgfältig plant, kann diese Methode auch in Ländern wie China wirksam sein.

Zur allgemeinen institutionellen Rahmenbedingung des DaF-Unterrichts in China liefert schon Kapitel 2 einen Überblick. Wegen der Weite des Landes und der großen Bevölkerungszahl zeigt der Fremdsprachenunterricht in China allerdings starke regionale Unterschiede (vgl. Wang 2007, S. 83). In der vorliegenden Arbeit wird das regionale Spezifikum berücksichtigt. Der Unterrichtstyp „Hochschuldeutsch“ und regionale Besonderheiten des Lernumfelds werden zunächst ausführlich dargestellt. Ihre Entwicklung, Zielgruppen und Rahmenbedingungen werden diskutiert, sowie spezielle Probleme dieses Unterrichtstyps. Die Besonderheiten des Fremdsprachenunterrichts in Westchina werden diskutiert, wobei die regionalen Rahmenbedingungen der empirischen Untersuchung herauskristallisiert werden. Danach werden das Forschungsdesign, die Methodik und die Zielgruppe vorgestellt, um die Herangehensweise der vorliegenden Untersuchung zu veranschaulichen. 


\subsection{Lernumfeld: Studienbegleitender Deutschunterricht an Hochschulen in Westchina}

Betrachtet man die Entwicklung des institutionellen Deutschunterrichtes in China (siehe Kapitel 2.1), kann festgestellt werden, dass es verschiedene Kursangebote gibt. Es gibt zwei wesentliche Ausbildungsarten des Deutschunterrichts an chinesischen Hochschulen: zum einen als Hauptfach für Germanistikstudierende und zum anderen als Nebenfach für Nichtgermanistik-Studierende (vgl. Zhu 2003, S. 35). Unter Deutsch als Nebenfach gibt es noch eine Reihe von unterschiedlichen Unterrichtsangeboten, die von verschiedenen Forschenden unterschiedlich kategorisiert werden (vgl. Zhu 2003, S. 35; Hess 2001, S. 1581; Hernig 2010, S. 1638; Li und Lian 2017, S. 125). In der vorliegenden Arbeit werden die aktuell üblichen Kursangebote wie folgt gegliedert:

- Hochschuldeutsch als 1. Fremdsprache: studienbegleitendes Pflichtfach für Studierende von Nichtfremdsprachenfächern wie Natur- und Ingenieurswissenschaften. Die deutsche Sprache dient für diese Zielgruppe als die erste Fremdsprache an der Universität.

- Hochschuldeutsch als 2. Fremdsprache: Als Pflicht- oder Wahlpflichtfach für Studierende von Fremdsprachenfächern wie Anglistik, sie dient hier als die zweite Fremdsprache.

- Deutsch als Wahlpflichtfach für alle Studierende: Der Unterricht findet normalerweise außerhalb der normalen Unterrichtszeit statt, das heißt am Abend oder am Wochenende und dauert nur ein Semester. Alle Studierenden können sich dafür anmelden.

- Intensivkurs für eine Weiterbildungsmöglichkeit in deutschsprachigen Ländern. Meistens handelt es sich hierbei um eine Prüfungsvorbereitung, beispielsweise der DaF-Vorbereitungskurs.

Für die vorliegende Forschung ist eine Analyse der Situation des studienbegleitenden Deutschunterrichts für Studierende von Nichtfremdsprachenfächern (Hochschuldeutsch als 1. Fremdsprache) von Bedeutung, der oft als „Deutsch als „Anwendungsfach“ oder „Hochschuldeutsch“ bezeichnet wird (vgl. Gu et al. 2003, S. 87; Zhu 2019, S. 9f., 2007a, S. 142; Li und Lian 2017, S. 127). Hier wird „Hochschuldeutsch“ als Bezeichnung dafür genommen. In manchen Statistiken wird allerdings keine klare Trennung von Hochschuldeutsch als 1. Fremdsprache und 2. Fremdsprache gemacht, weil es in der vorhandenen Literatur keine klare Differenzierung gibt ${ }^{10}$.

10 Beide Gruppen können an den nationalen Hochschuldeutschprüfungen Stufe 4 und Stufe 6 teilnehmen. 
Nach Hess (2001, S. 1581) bilden die studienbegleitenden DeutschunterrichtLernenden die größte Deutschlerngruppe an chinesischen Universitäten. Laut einer nationalen Statistik zum Hochschuldeutschunterricht im Jahr 1995 (Zhu 2009, S. 642f.) gibt es damals 116 Universitäten, die Hochdeutsch anbieten. Die Anzahl der damals deutschlernenden Studierenden beträgt 16 460. Im Jahr 2015 nahmen Studierende aus 232 Universitäten an der landeseinheitlichen Prüfung Hochschuldeutsch Stufe 4 und Stufe 6 teil (vgl. Zhu 2019, S. 10). Schätzungsweise besuchen jetzt 25000 Studierende an 300 Universitäten den Hochschuldeutsch-Unterricht (vgl. Li und Lian 2017, S. 127).

Die große Zielgruppe zeigt deutlich, dass dieser Unterrichtstyp eine wichtige Rolle für die deutsche Sprache in China spielen soll. Allerdings wird „Hochschuldeutsch" bisher nur sehr begrenzt in China erforscht, weil der Forschungsschwerpunkt im Deutsch als Hauptfach liegt (vgl. ebenda, S. 126f.). Es scheint so zu sein, dass Professor Zhu Jianhua, der (ehemalige) Vorsitzender des Anleitungskommitees für Hochschuldeutsch des chinesischen Bildungsministeriums der einzige Forscher ist, der in den letzten Jahren stets Beiträge zur Entwicklung des Faches veröffentlicht (vgl. 2003; Zhu 2007b, 2007a, 2009, 2019).

Studienbegleitender Deutschunterricht ist von Natur aus nicht einfach, weil er von vielen Faktoren beeinflusst wird und verschiedenen Rahmenbedingungen unterliegen kann. Diese Problematik fand allerdings viele Jahre keine Beachtung im DaF-Bereich und gewinnt nur in der letzten Zeit einige Aufmerksamkeit (vgl. Rösler 2015, S. 7).

Rösler (2015) deutet an, dass studienbegleitender Deutschunterricht von verschiedenen Faktoren geprägt ist. Dazu zählen Motivation der Lernenden, die Ausgangslage der Universitäten, die Lehrenden, Anzahl der Kontaktstunden, verschiedene Lerninhalte bzw. Gewichtung von Fachsprache und Kommunikation im Unterricht sowie die Beziehung zwischen Fachstudium und Spracherwerb. Er pointiert, dass „es den studienbegleitenden Deutschunterricht nicht gibt und nicht geben kann" (ebenda, S. 16). Der Bericht von Gu (2003) zeigt auch, dass an verschiedenen Universitäten in China unterschiedliche Motivationen und Lernziele im studienbegleitenden Deutschunterricht festgestellt werden können.

Im Folgenden wird deswegen versucht, zuerst eine allgemeine Situation des Hochschuldeutschunterrichts in China zu liefern und danach die regionale spezifische Eigenschaft des Fremdsprachenunterrichts in Westchina darzustellen. 


\subsubsection{Studienbegleitender Deutschunterricht: Situation von „Hochschuldeutsch“}

Der Ursprung von Deutsch als studienbegleitendes Fach an Hochschulen kann bis zum Anfang des 20. Jhd. zurückgeführt werden. Einige Universitäten bzw. Hochschulen bieten damals schon Deutschkurse außerhalb der fachlichen Lehrveranstaltungen an. An manchen Universitäten sind deutsche Lehrende für Fachunterricht tätig, und die deutsche Sprache ist die Unterrichtssprache (vgl. Zhu 2009, S. 628; Reinbothe 2007, S. 59-62). Vom Aufbruch des Krieges bis zum Ende der Kulturrevolution bleibt Hochschuldeutsch unentwickelt. Nach dem Krieg wird die deutsche Sprache zuerst wegen der starken Betonung von Russisch zum Mauerblümchen. Danach erschüttert die Kulturrevolution die ganze Fremdsprachenausbildung in China (vgl. Zhu 2009, S. 629-631).

Im Jahr 1980 wird das „Curriculum für öffentlichen Deutschunterricht“ herausgegeben. Das bedeutet, dass Deutschunterricht als studienbegleitendes Fach des Hochschulbildungssystems der VR China etabliert ist (vgl. ebenda, S. 631f.). Im Jahr 1985 wird „Öffentlicher Deutschunterricht" nach der Bezeichnung von "College English“ umbenannt: die Bezeichnung „College German“ gilt bis heute (vgl. Zhu 2007a, S. 141f.). Seitdem werden das Curriculum für Hochschuldeutsch und die Prüfungsordnung für Hochschuldeutsch Stufe 4 und Stufe 6 mehrmals überarbeitet. Es gibt nationalweit immer mehr Hochschulen, die Hochschuldeutsch als 1. und 2. Fremdsprache anbieten, und immer mehr Studierende, die an den nationalen Prüfungen teilnehmen. ${ }^{11}$

Angesichts der Globalisierung und dem Aufbau eines Wirtschaftsgürtels entlang der Seidenstraße von China nach Europa („Ein Gürtel, Eine Straße“) seit dem Jahr 2013 gewinnen viele Fremdsprachen neue Bedeutung (vgl. Li und Lian 2017, S. 121). Die Nachfrage nach Fremdsprachenausbildungen an Hochschulen ändert sich: Der Bedarf an Fachkräften mit genügendem fremdsprachlichem Wissen und Kommunikationskompetenz ist größer geworden. Die Ausbildung von Nicht-Englisch-Fremdsprachen an Hochschulen entspricht wenig dem Marktbedarf und dem Entwicklungsbedarf des Landes (vgl. Xu 2017, S. 45f.; Peng und Yang 2018, S. 190). Auch die deutsche Sprache gewinnt mehr an Bedeutung als früher (vgl. Peng und Yang 2018, S. 190; Zhu 2019, S. 10). Die wachsende Anzahl von Deutschlernenden in China zeigt dies (vgl. Auswärtiges Amt 2015, S. 7).

11 Im Jahr 1995 waren 1012 Studierende aus 17 Hochschulen von 8 Provinzen, im Jahr 2015 waren 9404 Studierende aus 232 Hochschulen von 25 Provinzen (Es gibt 34 Provinzen in China) (vgl. Zhu 2019, S. 10). 
Studienbegleitender Deutschunterricht soll unter diesem Kontext als besonders wichtig betrachtet werden, weil Hochschuldeutsch die sprachliche Ausbildung für Nicht-Fremdsprachenfächer anbietet. Eine Kombination von dem Hochschuldeutschunterricht und der fachlichen Lehrveranstaltungen entspricht dem Marktbedarf im Rahmen von „Ein Gürtel, eine Straße“. Allerdings trifft die Entwicklung von studienbegleitendem Deutschunterricht an chinesischen Hochschulen auf eine Reihe von Schwierigkeiten. Li (2009, S. 256) ist der Meinung, dass studienbegleitender Fremdsprachenunterricht nie die genügende Aufmerksamkeit bekam, und nur eine sehr begrenzte Anzahl von Studierenden durch studienbegleitenden Fremdsprachenunterricht genügendes Wissen ihrer Zielsprache bekommen können.

Erstens fehlt es an hochqualifizierten Lehrenden. An viele Universitäten und Hochschulen sind nur 1-2 Lehrende für Hochschuldeutsch tätig (vgl. Zhu 2019, S. 12), wie z. B. an der Technischen Universität Kunming. Neben dem Lehrkräftemangel wird der Unterricht oft in großen Gruppen erteilt, von 35 bis 100 in einer Klasse (vgl. Peng und Yang 2018, S. 191; Zhu 2019, S. 15). Außerdem ist die Anzahl der Kontaktstunden gering, nach Zhu (2019, S. 16) variiert die Gesamtzahl von 240 bis 280 . Fehlende Lehrkräfte, große Gruppen und begrenzte Kontaktstunden führen dazu, dass Frontalunterricht in der Praxis die Hauptrolle spielt.

Spricht man von der Qualifikation von Lehrenden für Hochschuldeutschunterricht, muss man zuerst herausfinden, welche Lernziele der Unterricht hat. Das Hauptlernziel für Hochschuldeutsch ist sprachliches Grundwissen, was dem Bedarf des Marktes aber nicht völlig entspricht (vgl. Peng und Yang 2018, S. 191). Obwohl in dem überarbeiteten Curriculum außer „notwendige Sprachkenntnisse" auch Kommunikationsfähigkeit und interkulturelle Kompetenz betont werden (vgl. Zhu 2019, S. 12f.), muss man viel Zeit auf sprachfundamentales Wissen verwenden, wenn man die Prüfung für Hochschuldeutsch Stufe 4 und Stufe 6 bestehen will. Da in der Prüfung keine mündliche Kommunikation getestet wird, wird der mündliche sprachliche Aspekt oft vernachlässigt. Die Evaluation von Fremdsprachen zeigt ein großes Problem im chinesischen Bildungssystem, das man kurzfristig nicht verändern kann (vgl. Zheng 2018, S. 49).

Außerdem sollen folgende Fragen auch beantwortet werden: Soll die Fachsprache dabei eine Rolle spielen, wenn ja, in welcher Tiefe? Sollen die Lehrenden außer sprachlichem Wissen auch fachliches Wissen (der natur- und technischen Wissenschaften) beherrschen? Wie sieht die Gewichtung von Kommunikationsfähigkeit und interkultureller Kommunikation aus? Wie sieht die Zielgruppe des Unterrichts aus? 
Diese Reihe von Fragen lässt feststellen, dass die Planung eines guten studienbegleitenden Deutschunterrichts von vielen Schwierigkeiten behindert werden kann. Der Hochschuldeutschunterricht, den die meisten Universitäten anbieten können, besteht aus einfachen Sprachkursen und entspricht den Kriterien eines richtigen studienbegleitenden Deutschunterrichts von Rösler (2015) nicht.

\subsubsection{Zur Bildungsungleichheit zwischen Ost- und Westchina}

Hochschuldeutsch in China - hinter der gleichen Bezeichnung können sich verschiedene Rahmenbedingungen und Zielgruppen verbergen. Die Bildungsungleichheit in China kann dabei eine Rolle spielen. Während die Ostregionen von China entlang der Westküste des Pazifik in den letzten Jahren ein großes ökonomisches Wachstum erzeugt haben, ist die Entwicklung der Mittel- und Westregionen vergleichsweise begrenzt. Der ökonomische Unterschied verursacht eine große Diskrepanz auf der Bildungsebene in verschiedenen Regionen (vgl. You und Dörnyei 2016, S. 496; Hu 2017, S. 16; He 2008, S. 1).

Nach der Untersuchung von $\mathrm{Hu}(2017$, S. 16) über regionale Unterschiede des Bildungswesens von 31 Provinzen in China ist die Bildungszeit von den Bürgern im Osten am längsten und im Westen am kürzesten. Außerdem ist das Bildungsniveau zwischen Stadt und Land in Ostchina deutlich besser als das der Mittel- und Westregion. Für die vorliegende Arbeit ist die Bildungssituation von Westchina und deren Unterschied zum Osten von Bedeutung und wird hier veranschaulicht.

Zu Westchina zählt man Shanxi, Xinjiang, Gansu, Qinghai, Ningxia, Sichuan, Chongqing, Yunnan, Guizhou, Xizang (Tibet) und den Westen von Hunan. Die geographische Besonderheit begrenzt die Entwicklung der Regionen, weil ihre Mehrheit von hochliegenden Gebirgen umgeben ist. Außer Han-Chinesen leben noch 55 Minderheitsnationen in China, 43 davon wohnen in Westchina. Aufgrund der spezifischen Naturbedingungen und aus historischen Gründen liegt die ökonomische und soziale Entwicklung der Region weiter zurück von der in der Ost- und Mittelregion (vgl. He 2008, S. 1; Qi 2017, S. 5; Wang 2013, S. 52f.).

$\mathrm{Hu}$ (2008, S. 48f.) deutet an, dass die Schwachstelle der Bildung in Westchina eine fehlende humanistische Ausbildung ist. Der Lerninhalt an der Schule vernachlässigt die humanistische Ausbildung und den kulturellen Hintergrund von Minderheitsnationen. Ein anderes Problem der Bildung in Westchina ist der Lehrkräftemangel. Die Anzahl und die Qualifikation der Lehrenden können den Bedarf vor Ort nicht befriedigen. Qi (2017, S. 5) ist der Meinung, dass die ungünstige Verkehrsinfrastruktur die Ursache ist. Gut ausgebildete Lehrende wollen nicht fürs ganze Leben tief in den Bergen bleiben. Lehrerausbildung kann 
eben wegen der Verkehrsprobleme nicht systematisch und regelmäßig durchgeführt werden.

Nach dem Entwicklungsbericht von Westchina im Jahr 2013 zeigen die Hochschulen in Westchina einigermaßen Fortschritte, allerdings im Vergleich zu Hochschulen in Mittel- und Ostchina nur gering. Die Provinz Sichuan, Shanxi und Chongqing sind etwas besser entwickelt, während die übrigen Regionen noch relativ schwach sind. Es gibt zahlreiche Probleme im Grundausbildungsbereich. Wegen fehlender Gleichberechtigung von Frauen und Männern besuchen viele Mädchen in den extrem armen Regionen keine Schule. Die Analphabetenquote in den Armenregionen in Westchina ist hoch. Dazu kommt noch die rückständige Einstellung gegenüber Bildung, schlechte Lernbedingungen und fehlende Lehrkräfte (vgl. Qi 2017, S. 5; Wang und Yang 2017, S. 143). Auch die Lehrkraftsituation im Hochschulbereich von Westchina ist im Vergleich zu der in Mittel- und Ostchina relativ unbefriedigend (vgl. Wei und Liu 2016, S. 79f.).

Die Bildungsdiskrepanz existiert nicht nur zwischen Ost- und Westchina, sondern auch zwischen Stadt und Land, da die Stadt bessere Lehrkräfte einsetzen und Städter stärker ausgeprägte Bildungswünsche haben (vgl. You und Dörnyei 2016, S. 496). Die Bildungschancen, die Bildungsinvestitionen und Bildungsbedingungen sind anders (vgl. Hu 2017, S. 14). Während die Städte, insbesondere die Hauptstädte, relativ gute Ausbildungsbedingungen besitzen, ist die Ausbildung auf dem Land oft problematisch. Die Bildungsungleichheit zwischen Stadt und Land in Westchina ist höher als die in Ostchina (vgl. ebenda, S. 17). Allerdings leben 80 \% der Bevölkerung von Westchina auf dem Land (vgl. Qi 2017, S. 5). Das heißt, dass die Mehrheit der Bevölkerung von Westchina unter schlechten Bildungsbedingungen leben.

Ein großes Problemen der Bildung in Westchina stellt die fehlende fremdsprachliche Ausbildung dar. In der Grundausbildung der Fremdsprachenerziehung können große Unterschiede zwischen Ost und West bezüglich der Lehrkräfte, der Lernbedingung und Lernqualität und dem Stellenwert gegenüber Fremdsprachen in der jeweiligen Region festgestellt werden (vgl. Li 2010, S. 34).

Auch bei der Fremdsprachenausbildung im Hochschulbereich kann man große Unterschiede zwischen Ost und West finden. Wenn man die Liste von Universitäten und Hochschulen mit Germanistikabteilungen (vgl. Jia und Wei 2011, S. 179-184) betrachtet, kann man feststellen, dass nur 5 Universitäten aus den westlichen Regionen (konkret aus Shanxi, Sichuan und Chongqing) eine Germanistikabteilung haben, während insgesamt 68 Universitäten auf der Liste aufgeführt werden. 
An der Mehrheit der Schulen in den armen Westregionen gibt es auch keine qualifizierten Englischlehrenden (vgl. E 2013, S. 63), und die Leistung der Schüler im Fach Englisch ist auch unbefriedigend im Vergleich zu denen aus dem Osten (vgl. You und Dörnyei 2016, S. 496; Hu 2003, S. 14f.). Das Fach Englisch wird oft von Chinesisch- oder Mathematik- Lehrenden gelehrt, die selber sehr begrenzte Englischkenntnisse haben (vgl. Jiang 2012, S. 7f.; Li 2010, S. 35). ${ }^{12}$

Jiang (2012) analysiert den „Eco-Context" und „Eco-Compensations" der Fremdsprachenausbildung in Westchina und findet als ungünstige Bedingungen für die Fremdsprachenausbildung den niedrigen Stellenwert von Fremdsprachen auf dem Land und die Multikulturalität vor Ort. Fremdsprachen zu beherrschen wird auf dem Land in Westchina nicht als wichtige Fähigkeit anerkannt wie in Ostchina, das stärker von der Globalisierung geprägt ist (vgl. Hu 2003, S. 15). Lehrkräftemangel führt dazu, dass das Fach Englisch an der Schule in armen Regionen keine Rolle spielt, weil man Wert auf Chinesisch und Mathematik liegt. Das Erlernen von Fremdsprachen ist prüfungsorientiert. Die Menschen dort verspüren wenig Neigung zu humanistischem Wissen.

Außerdem haben viele chinesische Minderheitsnationen ihre eigene Sprache. Das heißt, dass Englisch die zweite Fremdsprache für sie ist, da Mandarin die erste Fremdsprache sein soll. Das Erlernen von Chinesisch soll fürs Leben und die Erziehung im Vordergrund stehen (vgl. Luo 2001). Wenn es überhaupt Englischlehrkräfte gibt, sind es überwiegend Han-Chinesen, die keine minderheitsnationale Sprache sprechen können (vgl. Jiang 2012, S. 6; Hu 2003, S. 16).

Die fehlende Fremdsprachenausbildung an Grund- und Mittelschulen hat Konsequenzen für die Fremdsprachenausbildung an Hochschulen. Die Forschung von You und Dörnyei (2016, S. 516f.) zeigt, dass Studierende in Westchina eine deutlich niedrige Motivation beim Englischlernen im Studium als die in Ostchina zeigen. Sie begründen dies so:

The more advanced or specialized one's education was, the stronger ideal language image the student entertained. A likely explanation for this trend is that the students' education increasingly opens up their horizon onto the global world beyond China, bringing about an increasing appreciation of Global English (ebenda, S. 517).

12 Eine Erfahrung der Autorin als Beispiel für den Extremfall: Laut einer Deutschen, die mit ihrer Familie für lange Zeit in einem Dorf den Englischunterricht am Grenzgebiet von Provinz Yunnan übernimmt, gibt es auf der Dorfschule 3 chinesische EnglischLehrende, die kein Englisch beherrschen. Die gelehrte Sprache ist eine selbst entwickelte Sprache jeder Lehrkraft. Jede lehrt deswegen auch ein anderes "Englisch". 
Eine Anglistikstudentin aus Westchina berichtet in einer Untersuchung der Bildung in Westchina, dass sie im Vergleich mit Studierenden aus der Ostregion größere Schwierigkeiten beim Englischlernen hat und ein niedrigeres Niveau der Englischausbildung erreicht (besonders bei der Aussprache), weil sie schon bei der Grundbildung schlechtere Ausbildungschancen hatte (vgl. E 2013, S. 63).

\subsubsection{Zur Situation der Fremdsprachenausbildung in der Provinz Yunnan}

Die vorliegende Forschung findet in der Provinz Yunnan statt, die zu Westchina gehört. Die Provinz Yunnan liegt an der Grenze zwischen China, Vietnam, Laos und Birma, hat eine Fläche von $394000 \mathrm{~km}^{2}$. Geographisch ist Yunnan ein Hochland. 94 \% der Provinz ist gebirgig (vgl. Fei et al. 2016, S. 67). Die geographische Bedingung führt zur Multikulturalität in der Provinz (vgl. He 2008, S. 70f.).

In Yunnan leben 25 Minderheitsnationen neben Han-Chinesen, davon leben 15 nur in der Provinz Yunnan. Die Minderheitsnationen nehmen 33,4 \% der gesamten Bevölkerung der Provinz ein (vgl. Die Regierung der Provinz Yunnan 2017a, S. 1). Yunnan besitzt eine der größten Minderheitsbevölkerungen unter allen chinesischen Provinzen (vgl. Fei et al. 2016, S. 68; Die Regierung der Provinz Yunnan 2017a, S. 1). 23 Minderheitsnationen davon pflegen ihre eigene Sprache (vgl. Die Regierung der Provinz Yunnan 2017b). Eine Statistik aus dem Jahre 2012 (vgl. Zhao und Mao 2012, S. 224) verdeutlicht, dass über 7 Millionen der Bevölkerung in Yunnan kaum Mandarin beherrschen und über 10 Millionen der Minderheitsbevölkerung in ihrer minderheitsnationalen Muttersprache kommunizieren ${ }^{13}$.

Die Provinz Yunnan ist einerseits bekannt für ihre schöne Landschaft, das gute Klima und die kulturelle Vielfalt, ist andererseits aber eine der ärmsten Provinzen in China. Bis Ende 2015 gibt es noch über 5 Millionen Bevölkerung, die unter Armut leiden. Die Anzahl der unter Armut leidenden Bevölkerung von

13 Der zitierte Beitrag wurde im Jahr 2012 veröffentlicht. Die Daten können aus noch früherer Zeit stammen und zeigen nur die Situation vor 10 Jahren. Andere Quelle zum Mandarin-Gebrauch der Minderheitsnationen in der Provinz Yunnan in der jüngeren Zeit liegen $\mathrm{m}$. W. leider nicht vor. Es lässt sich aber vermuten, dass die rasende Entwicklung von Internet und Smartphone die Rolle der Mandarin-Sprache in Minderheitsnationen-Regionen verändert haben. Auch die Bildungsentwicklung dürfte den Mandarin-Gebrauch in den letzten Jahre stark vorangetrieben haben, sodass vermutlich inzwischen mehr Menschen Mandarin beherrschen als vor 10 Jahren. 
Yunnan ist die höchste unter allen chinesischen Provinzen (vgl. Li et al. 2018, S. 24).

Auch das Bildungsniveau in Yunnan sieht nicht gut aus. Mangelnde Gleichberechtigung zwischen Männern und Frauen sowie fehlende Lehrkräfte an Schulen, besonders in den Grenzregionen, sind keine Seltenheit (vgl. Wang und Yang 2017, S. 143f.; Zhao und Mao 2012, S. 223f.). Die geographische Lage macht es schwer, in Yunnan Autobahnen und Eisenbahnstrecken zu bauen (vgl. Fei et al. 2016, S. 68). Im Extremfall gibt es im Dorf überhaupt keine ordentliche Straße. Die Gebirge bilden eine starke Barriere für den Aufbau der Infrastruktur und führen zu problematischen schulischen Verhältnissen. Manche Schüler in den Bergen müssen täglich viele Kilometer zur Schule laufen (vgl. Zhao und Mao 2012, S. 223).

Hinsichtlich der Fremdsprachenerziehung zeigt Yunnan folglich wie andere Westregionen große Unterschiede im Vergleich zu Ostchina. Luo (2012, S. 112114) analysiert den Bildungshintergrund von 101 Englischlehrenden an Grundschulen in der Provinz und stellt fest, dass nur $36 \%$ davon Anglistik studierten und die Mehrheit davon keinen Bachelor-Abschluss haben.

Auch im Hochschulbereich bleibt Yunnan weiter zurück als die anderen Provinzen in China. Die Entwicklung der Hochschulen kann auf die Zeit des Zweiten Weltkriegs zurückgeführt werden, als die Eliteuniversitäten von ostchinesischen Städten wegen des Krieges nach Yunnan umgezogen sind. Die Hochschulbildung in Yunnan entwickelt sich seitdem langsam. Bis Jahr 2017 gibt es in Yunnan 77 Hochschulen, davon 32 Universitäten (vgl. Ministerium für Bildung der VR China 2018). Es gibt nur eine Universität, die Universität Yunnan, die als Schwerpunktuniversität in China eingestuft wird.

Die Entwicklungsungleichheit innerhalb der Provinz kann man anhand der Anzahl der Universitäten feststellen: Im Jahr 2015 gibt es in der Hauptstadt Kunming 20 Universitäten, während es in anderen Teilen der Region insgesamt nur 8 gibt (vgl. Cao 2015, S. 13f.). Typisch für Westchina: Die Hochschulen und Universitäten befinden sich meistens in den Hauptstädten der Provinzen. Die Ungleichheit der Verteilung innerhalb einzelner Provinzen ist offensichtlich (vgl. Wei und Liu 2016, S. 79).

Das Problem der Bildungsdiskrepanz zwischen Stadt und Land wird seit Jahren von der chinesischen Regierung erkannt. Verschiedene Maßnahmen werden ergriffen, um die Situation zu verbessern. Seit dem Jahr 1999 erweitert man die Zulassungsquote an Hochschulen, sodass sich die Hochschulbildung von einer Elitebildung zur Massenbildung wandeln kann und mehr Landbevölkerung die Chance auf eine Hochschulbildung bekommen können (vgl. Chang et al. 2018, 
S. 78). Da der große Teil der Bevölkerung in China auf dem Land lebt, ist die Maßnahme für eine Verbesserung der Bildung von Bedeutung.

Die Wirkung dieser Maßnahme ist allerdings auch kritisch zu sehen. Chang (2018, S. 85f.) analysiert die Zulassungsquote im Jahr 1997 und 2011 und stellt heraus, dass sich die Zulassungsquote der Landbevölkerung zu Hochschulen deutlich erhöht. Während im Jahr 1997 nur 5,12 \% Landbevölkerung eine Bildungschance an der Hochschule haben, bekommen 20,74 \% im Jahr 2011 eine Zulassung. Die Diskrepanz der Bildungszeit zwischen Stadt und Land ist kleiner geworden. Aber zwischen der Zulassungsquote von Stadt und Land gibt es immer noch einen sehr großen Unterschied. Ein Bericht im Jahr 2011 (Lin 2011, S. 1) zeigt, dass unter 1 Million Schüler, die die Hochschulaufnahmeprüfung im Jahr 2010 aufgegeben haben, Schüler auf dem Land den größten Anteil haben.

Zusammenfassend lässt sich sagen: Die Diskrepanz zwischen Stadt und Land wird nicht kleiner, obwohl die Bildungschance für die Landbevölkerung tatsächlich verbessert wird. Die erweiterte Zulassung hat nämlich mehr Bildungschancen für die Stadt als für das Land gebracht (vgl. Meng et al. 2017, S. 15; Chang et al. 2018, S. 86).

Basierend auf einer Untersuchung im Jahr 2012 gehört Yunnan zu einer der Provinzen, deren Zulassungsquote an Schwerpunktuniversitäten ${ }^{14}$ niedriger als der nationale Durchschnitt ist (vgl. Zhang und Zhang 2015, S. 29f.). Die Zulassungsquote von Schülern aus der Provinz ist hoch an lokalen Universitäten (vgl. Jiang et al. 2013, S. 189), weil Hochschulen für die Bildungsgleichheit der eigenen Region beitragen sollen (vgl. Zhang und Zhang 2015, S. 35). Die Technische Universität Kunming zum Beispiel hat 539 Bewerber aus armen Familien in der Provinz Yunnan im Jahr 2018 aufgenommen. 1529 Immatrikulierte haben minderheitsnationalen Hintergrund und nehmen ca. 19,2 \% der gesamten Immatrikulierten ein (vgl. Technische Universität Kunming 23.08.2018). Wegen der fehlenden Fremdsprachenausbildung in der Schulbildungszeit verfügen viele Studierende aus dem Land nur über begrenzte Fremdsprachenkenntnisse (Englisch) und haben deswegen große Schwierigkeiten im Fremdsprachenunterricht des Studiums (vgl. Jiang et al. 2013, S. 189).

14 Unter Schwerpunktuniversität versteht man hier als "211 Hochschulen". Es gibt in China bis zum Jahr 2015 insgesamt 112 Schwerpunktuniversitäten (vgl. Zhang und Zhang 2015, S. 29). 


\subsubsection{Schlussfolgerung zum Lernumfeld}

Basierend auf der Darstellung der Bildungssituation in Westchina bzw. in der Provinz Yunnan und die Diskussion über die Besonderheit von studienbegleitendem Deutschunterricht kann man feststellen, dass diesem an Universitäten in Westchina eine spezielle Problematik innewohnt.

Erstens haben die Studierenden aus der Region relativ ungünstige Bildungserfahrung hinsichtlich des Fremdsprachenlernens. Die fehlende Ressource und mangelhafte Lehrsituation führen dazu, dass sie schlechte Lernerfahrungen vom Fremdsprachenunterricht mitbringen und auch nur über begrenztes Wissen zu den Fremdsprachen verfügen. Sie haben mehr Schwierigkeiten im Fremdsprachenunterricht als die ostchinesischen Studierenden.

Jiang und ihre Forschungsgruppe (2011a; 2013; 2012; 2011b) führten eine Reihe von Untersuchungen über die Situation des Englischlernens ländlicher Bachelor-Studierenden an einer Universität in der Hauptstadt Kunming durch. Die Untersuchung zur Motivation zeigt, dass, im Vergleich zu Studierenden mit städtischer Herkunft, die Studierenden aus dem Land beim Fremdsprachenlernen mehr extrinsisch und weniger intrinsisch motiviert sind: Ihnen fehlt es an Selbstvertrauen und Interesse am Englischlernen. Ihre Eltern zeigen auch kein Interesse an ihrer Fremdsprachenfähigkeit. Sie haben, genau wie die StadtStudierenden, ein stark prüfungsorientiertes Lernziel. Für sie ist Englisch nur als Voraussetzung für weitere Prüfungen wichtig (vgl. Jiang et al. 2011a, S. 24). Außerdem kann man feststellen, dass Studierende aus dem Land kaum selbständig außerhalb des Unterrichts lernen und unbefriedigende Leistung bei Prüfungen haben, besonders beim Hörverständnis, Leseverständnis und Schreiben (vgl. Jiang et al. 2013, S. 189).

Zweitens könnte die geographische Lage in Yunnan den Stellenwert von Fremdsprachen beeinflussen. Während die Ostregionen durch Globalisierung viele europäische bzw. nordamerikanische Einflüsse erleben können, zeigt die Globalisierung in Yunnan eine südasiatische Orientierung (vgl. Zhao und Zhang 2017, S. 38f.). Das heißt, dass in Yunnan mehr Wert auf südasiatische Fremdsprachen gelegt wird als auf europäische Sprachen wie Deutsch. Im Rahmen von „Ein Gürtel, Eine Straße“ soll Yunnan Thailändisch, Vietnamesisch, Indonesisch u.a. Sprachen von Südostasiatischen Ländern fördern, da Yunnan als „Brückenkopf“ zwischen China und Südostasien gilt (vgl. Feng et al. 2013, S. 145). Laut der Untersuchung zum Fremdsprachenbedarf von Zhao und Zhang (2017, S. 39f.) in drei Grenzstädten von Yunnan, nimmt dabei Englisch die erste Stelle ein, gefolgt von Vietnamesisch, Thailändisch, Birmanisch, Laotisch und Khmer(Amtssprache von Kambodscha). 
Außerdem zeigt die Untersuchung von Wei (2015, S. 214-217) über die Berufsauswahl von Hochschulabsolventen in der Provinz Yunnan, dass die Mehrheit der einheimischen Absolventen in wenig entwickelten kleinen Städten oder Kreisstädten arbeiten wollen. Die Absolventen, die nicht aus Yunnan stammen, arbeiten nach dem Studium in größeren Städten, während die Absolventen, die aus Yunnan stammen, mehrheitlich in Kreisstädten oder auf dem Land in Yunnan Stellen finden. Megastädte treffen die Berufserwartung und die Wirklichkeit der yunnanischen Studierenden offensichtlich nicht. In diesem Fall muss man klar feststellen, dass das Erlernen der deutschen Sprache für die Studierenden in Yunnan von geringer praktischer Bedeutung ist.

Schlechte Lernerfahrung, fehlende Grundausbildung beim Fremdsprachenlernen sowie mangelhafte Berufschancen bezüglich Deutsch führen dazu, dass der Deutschunterricht an Universitäten in der Hauptstadt Kunming andere Lernziele haben soll als an Universitäten in Megastädten von Mittel- oder Ostchina. Das nationale Curriculum für Hochschuldeutsch stellt für die Zielgruppe im Grunde eine Überforderung dar. Aus diesem Grund nehmen sie auch nicht an den nationalen Prüfungen der Stufe 4 und Stufe 6 teil.

Das Erlernen von Deutsch bietet für diese Studierenden eher die Möglichkeit, ihren Horizont zu erweitern, allgemein bessere Lernerfahrung mit Fremdsprachenunterricht $\mathrm{zu}$ machen, und Motivation für Fremdsprachenlernen zu entwickeln. Deswegen wird das Sprachlernspiel als Methode ausgewählt, um den Fremdsprachenunterricht für diese Zielgruppe zu verbessern.

\subsection{Forschungsdesign}

Der Ausgangspunkt der vorliegenden Forschung ist, den DaF-Unterricht an der Technischen Universität Kunming zu verbessern, die Schweigsamkeit und mangelnde Lernbereitschaft der Unterrichtzielgruppe zu verändern. Die Forschung wird in der Form einer Aktionsforschung durchgeführt.

Aktionsforschung (action research) wird auch als Handlungsforschung bezeichnet (vgl. Boeckmann 2016, S. 592; Caspari et al. 2007, S. 499). Sie wird von der Lehrperson in ihrem Praxisumfeld durchgeführt unter dem Ziel, eine Praxisverbesserung mit den „Erforschten“ gemeinsam anzustreben (vgl. Boeckmann 2016, S. 592; Grotjahn 2007, S. 496).

Feldmeier (2014, S. 255) ist der Meinung, dass Aktionsforschung besonders geeignet für Unterrichtsbereiche ist, die wenig untersucht werden. Feldmeier stützt sich darauf, dass die Lehrkräfte vor Ort „zuallererst“ die Notwendigkeit von Untersuchungen wahrnehmen können, die zur Unterrichtsverbesserung führen. 
Ein Vorteil der Aktionsforschung ist die hohe Praxisrelevanz. Die Forschung kann in der natürlichen Unterrichtssituation durchgeführt werden, sodass eine Fragestellung besonders unterrichtsrelevant erfolgen kann (vgl. ebenda, S. 257). Dabei bietet Aktionsforschung ergänzenden Zugang zu den Unterrichtsgegenständen, was konventionelle quantitative und qualitative Forschungen nicht leisten können (vgl. ebenda, S. 266). Außerdem können in der Aktionsforschung aber zusätzlich sowohl qualitative als auch quantitative Methoden eingesetzt werden (vgl. Boeckmann 2016, S. 594). Altrichter (1998, S. 13) merkt an, dass Aktionsforschung Lehrenden helfen kann, selbständig Praxisprobleme zu bewältigen und innovativ zu sein.

Hinsichtlich des methodologischen Standards wird jedoch Aktionsforschung wegen ihrer mangelhaften Möglichkeit der Verallgemeinerung kritisiert (vgl. Grotjahn 2007, S. 496). Es ist jedoch fraglich, ob konventionelle Forschungsmethoden eine bessere Generalisierbarkeit hervorbringen können. Mehrfache Wiederholungen von Untersuchungen innerhalb der Aktionsforschung können die Qualität sichern (vgl. Boeckmann 2016, S. 593).

Zusammenfassend lässt sich sagen, dass Aktionsforschung ein ideales Forschungsinstrument für das vorliegende Forschungsvorhaben ist. Der Autorin wird dadurch ermöglicht, gleichzeitig als Forschende und Lehrende den Forschungsgegenstand zu untersuchen. Aktionsforschung macht es eben möglich, sowohl quantitative als auch qualitative Datenerhebungsmethoden zu den verschiedenen Zeitpunkten einzusetzen, um die Forschung auf Methoden- und Datenebene zu triangulieren. Die Triangulierung wird als wichtige Methode angesehen, verschiedene Facetten des Fremdsprachenunterrichts umfassend zu berücksichtigen. Dadurch wird ein „angemessenes Bild“ des Untersuchungsgegenstands gewonnen und der Aussagewert der ermittelten Daten erhöht (vgl. Aguado 2014, S. 52; Caspari et al. 2007, S. 501).

Weil Aktionsforschung wiederholende Schritte (Forschungszyklus) erhalten kann, ist am Ende der Durchführung eines Forschungszyklus eine Reflexionsphase vorgesehen, um weitere Forschungsschritte zu planen. Nach der Datenanalyse des ersten Zyklus wird auf Grundlage der Ergebnisse entschieden, ob ein neuer Zyklus durchgeführt werden soll. In dem neuen Zyklus kann eine erneute Planung bzw. die Fortsetzung des Forschungsprozesses überlegt werden (vgl. Feldmeier 2014, S. 262). Altericht (1998) macht den Kreislauf einer Aktionsforschung mit der folgenden Abbildung deutlich: 


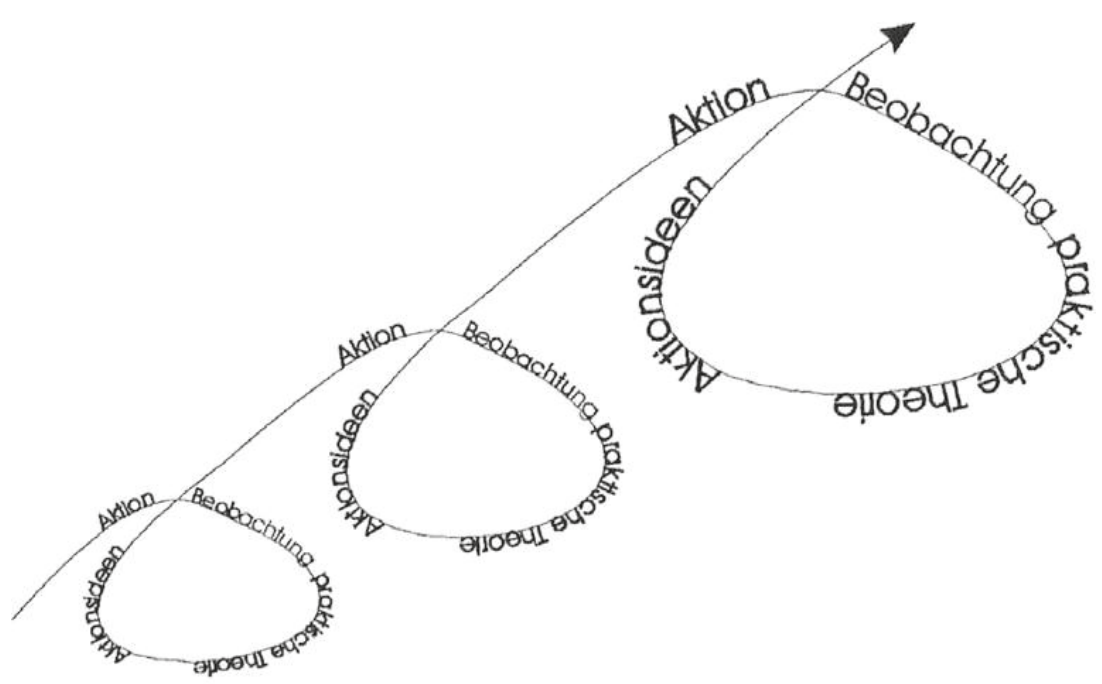

Abbildung 8: Der Kreislauf von Aktion und Reflexion (Altrichter und Posch 1998, S. 17)

Aufgrund der längerfristigen und zyklischen Charakteristik der Aktionsforschung (vgl. ebenda, S. 17) werden zwei Forschungszyklen und deren Ergebnisse in der vorliegenden Arbeit getrennt dargestellt und analysiert, wobei der zweite Zyklus bzw. Forschungsphase im Sommersemester 2017 aufgrund der Ergebnisse des ersten Zyklus (im Wintersemester 2016-17) geplant bzw. fortgesetzt wird. Die eingesetzten Datenerhebungsmethoden werden in einzelnen Unterkapiteln zu jeweiligen Forschungsphasen (siehe Kapitel 5.1.1 und Kapitel 5.2.1) veranschaulicht.

Im Folgenden werden der allgemeine Forschungsgegenstand, der organisatorische Hintergrund und die Forschungsmaterialien vorgestellt, um das gesamte Forschungsdesign darzustellen.

\subsubsection{Forschungsgegenstand}

Gegenstand der vorliegenden Studie ist die Effizienz von Sprachlernspielen. Übergeordnet ist dabei das didaktische Interesse, den Fremdsprachenunterricht in Ländern zu verbessern, wo man Schweigsamkeit und mangelnde Interaktion 
bei den Lernenden häufig beobachten kann. Die übergreifende Forschungsfrage lautet:

Können Lernende aus einer Lerntradition, die stark von der lehrerzentrierten Unterrichtsform und passivem Lernen geprägt ist, sich an interaktive/kommunikative Methoden wie das Sprachlernspiel gewöhnen und dabei bessere Leistungen und höhere Motivation als im lehrerzentrierten Unterricht zeigen?

Bei der Forschung soll herausgefunden werden, ob und gegebenenfalls wie das Sprachlernspiel die sprachlichen Kompetenzen sowie die Motivation der Lernenden verbessern und erhöhen kann.

Um die Erhöhung der Motivation zu überprüfen, werden folgenden Unterfragen gestellt:

1. Können die Lernenden mehr Selbstvertrauen beim Sprachlernen durch Einsatz von Sprachlernspielen gewinnen?

2. Verbessert sich die Einstellung der Lernenden gegenüber der Zielsprache bzw. Deutsch durch Sprachlernspiele?

3. Wird der Unterricht mit Sprachlernspielen besser als der Unterricht ohne Sprachlernspiele bewertet?

4. Sind die Lernenden im Unterricht mit Sprachlernspielen aktiver als die im Unterricht ohne Sprachlernspiele?

Die Verbesserung der sprachlichen Kompetenzen bzw. des Lernfortschritts werden mit folgenden Unterfragen berücksichtigt:

1. Können die Lernenden mit Sprachlernspielen in den durchgeführten Prüfungen und Tests bessere Leistungen als die Lernenden ohne Sprachlernspiele erreichen?

2. Können Sprachlernspiele bestimmte Fertigkeiten stärker fördern, beispielsweise eine bessere Fertigkeit im Hören?

Außerdem will die Forschung versuchen, eine praxisorientierte Verbesserungsmöglichkeit des chinesischen Fremdsprachenunterrichts an Universitäten zu finden. Probleme und Widerstände, die den Einsatz von Sprachlernspielen in der Praxis verhindern können, werden unter die Lupe genommen:

1. Kann der Unterricht mit Sprachlernspielen zeitlich genau so effizient sein wie Unterricht ohne Sprachlernspiele?

2. Kann man traditionelle Übungsformen als Sprachlernspiele adaptieren?

3. Kann man Sprachlernspiele in einer größeren Gruppe einsetzen, auch wenn die Rahmenbedingungen nicht ideal für den Spieleinsatz sind?

Es folgen zwei zugehörige Hypothesen: 
1. Lernende, die den DaF-Unterricht mit Sprachlernspielen besuchen, sind in einer vergleichbaren Situation signifikant mehr motiviert, als Lernende in einem lehrerzentrierten DaF-Unterricht ohne Sprachlernspiele.

2. Lernende, die den DaF-Unterricht mit Sprachlernspielen besuchen, entwickeln in einer vergleichbaren Situation bessere sprachliche Kompetenzen, als Lernende in einem lehrerzentrierten DaF-Unterricht ohne Sprachlernspiele.

Die in die Forschung einbezogenen Variablen sind die folgenden:

Die unabhängige Variable ist der Einsatz vom Sprachlernspiel.

Die abhängigen Variablen sind Lernerfolg und Motivation der Lernenden, operationalisiert als die Ergebnisse der Semesterprüfungen, C-Tests und der durchgeführten Befragungen usw.

In Bezug auf Kontrollvariablen ist festzuhalten: Alle Probanden sind auf dem gleichen fremdsprachlichen Niveau, aus technischen und naturwissenschaftlichen Fächern, im gleichen Jahrgang der Universität und haben kein Vorwissen in der deutschen Sprache. Englisch ist ihre erste bzw. bisher einzige Fremdsprache. Das Verhältnis von Frauen und Männern ist bei den Probanden ausgewogen. Die Untersuchung verzichtet auf Gender- Probleme zum Spieleinsatz. In Bezug auf Lerninhalt und Lernperson wird abgesichert, dass die Probanden mit dem gleichen Lehrwerk nach entworfenem Didaktisierungsplan von der gleichen Lehrperson, der Forscherin selbst, unterrichtet werden. Beide Gruppen nutzen identisch ausgestaltete Unterrichtsräume. Die Tests sowie Befragungen finden für alle Probanden in den gleichen Zeitphasen unter den gleichen Bedingungen statt. Die Befragungen sind anonym durchgeführt, sodass die Befragten wissen, dass diese keinen Einfluss auf ihre Benotung in den Prüfungen und Tests haben werden.

Hinsichtlich möglicher Störvariablen gilt: Aus organisatorischen Gründen sind die Stärke der Gruppen nicht identisch, eine Gruppe hat 36 Probanden und die andere 30 (siehe Kapitel 4.2.2). Die Zahl der Probanden können in zwei Semestern wegen Abwesenheit ${ }^{15}$ sowie aus anderen persönlichen oder organisatorischen Gründen variieren. Das Abschreiben bei Prüfungen kann nicht $100 \%$ verhindert werden. Außerdem ist es für ein Feldexperiment schwer zu kontrollieren, welche Kenntnisse außerhalb des Unterrichts erworben werden.

\subsubsection{Organisatorischer Hintergrund und Probandensituation}

Die Forschung findet an der Technischen Universität Kunming (TUK) statt. Die TUK wurde im Jahr 1954 gegründet und ist die größte Universität in der Provinz

15 Wenn ein Studierender mehr als 33 \% der gesamten Sitzungen in einem Semester abwesend ist, darf er nicht an den Prüfungen teilnehmen. 
Yunnan. Sie bietet 110 Bachelor- und 41 Masterstudiengänge in Natur-, Technik- und Geisteswissenschaften sowie Medizin an. Im Jahr 2018 studieren an der TUK 31,447 Bachelorstudierende und 11,143 Masterstudierende sowie Promovierende. Die TUK arbeitet mit mehr als 60 Hochschulen in den USA, Deutschland, Frankreich und Großbritannien international zusammen (vgl. Technische Universität Kunming 30.10.2018).

Das Studium an der TUK wird nach strukturierten Curricula organisiert, wie an den meisten Universitäten in China. Das heißt, dass die Studierenden keinen Stundenplan für sich frei bauen müssen bzw. können. Kursangebote werden nach einem Curriculum jeder Fakultät organisiert. Für Studierende in China, die kein fremdsprachliches Fach, beispielsweise Anglistik oder Germanistik, studieren, ist das Erlernen einer Fremdsprache (meistens Englisch) während des Studiums obligatorisch. Der (Englisch-)Kurs wird als „college English“ bezeichnet und wird von der Fakultät für Fremdsprachen und -kulturen für die ganz Universität angeboten.

Die neu Eingeschriebenen in Bachelor-Studiengängen an der TUK müssen nach der Einschreibung zunächst an einem Englisch-Einstufungstest teilnehmen. Diejenigen, die weniger als 40 Punkte (von möglichen 100) in der Prüfung bekommen haben, sollen „kleine Sprachen“"16 lernen, d. h. Deutsch, Japanisch oder Französisch statt Englisch. Die Studierenden, die mehr als 40 Punkte im Einstufungstest bekommen haben, dürfen im Prinzip nur Englisch lernen.

An dieser Stelle muss man besonders betonen, dass diese Verteilung keine Strafe und kein Zwang ist. Erstens können die genannten Studierenden wegen fehlender Englischkenntnisse dem Englischunterricht kaum folgen. Da manche von ihnen wegen mangelnder Ressourcen aus der Schulzeit nur sehr begrenzte Kenntnisse der Fremdsprache beherrschen, kann der „kleine Sprachen“-Unterricht den Studierenden eine zweite Chance bieten, eine neue Fremdsprache als richtige Anfänger zu erlernen. Außerdem können sie sich auch für Englisch ummelden, wenn sie kein Interesse für die andere Fremdsprachen haben - allerdings nur unter der Voraussetzung, dass die Kapazität von Englischklassen dafür ausreichend ist.

Insgesamt gibt es an der Fakultät von Fremdsprachen und -kulturen der TUK im Jahr 2016 zwei Deutschlehrerinnen, zwei Französischlehrerinnen und drei Japanischlehrerinnen. Für das WS 2016/17 stehen eine Deutschlehrerin (die Autorin), eine Französischlehrerin und zwei Japanischlehrerinnen für die Erstsemester zur Verfügung. Im WS 2016/17 sind ca. 7000 Studierende eingeschrieben.

16 „Gegenüber Englisch werden alle anderen Fremdsprachen heute oft als ,kleine Sprache bezeichnet." (Zhu und Li 2004, S. 97) 
Aufgrund der großen Anzahl werden die Studierenden aus organisatorischen Gründen je nach dem Fachgebiet in 4 Zonen aufgeteilt. Außer Englisch dürfen die Studierenden von Zone 1 und 2 zwischen Deutsch und Japanisch wählen, während die von Zone 3 und Zone 4 Französisch oder Japanisch lernen dürfen.

Die Studierenden schreiben sich in den Kurs an der Online-Einschreibungsplattform ein. Diejenigen, die weniger als 40 Punkte im Einstufungstest bekommen haben, können nur die kleine Sprache wählen (wegen der Plattformeinstellung). Wenn sie trotzdem Englisch lernen wollen, können sie sich in der ersten zwei Wochen bei den Lehrenden für einen Fachwechsel manuell eintragen.

An der TUK besteht eine Englisch-Klasse normalerweise aus 50 bis 60 Studierenden. Für die kleinen Fremdsprachen ist diese Gruppe etwas zu groß, weil die Lernenden richtige Anfänger sind. Außerdem darf eine Klasse im Prinzip nicht aus weniger als 30 Studierenden bestehen. Deswegen beträgt die Anzahl einer Kleine-Sprache-Klasse normalerweise aus 30 bis 50 Lernenden.

Die Anzahl der Studierenden, die beim Einstufungstest im WS 2016- 17 weniger als 40 Punkte bekommen haben, ist groß. In manchen kleinfremdsprachlichen Klassen sammeln sich 60 oder sogar noch mehr Studierende. Die Deutschklasse Zone 1 besteht am Semesteranfang aus 60 Studierenden, Deutschklasse Zone 2 aus 37 Studierenden. Es gibt viele, die sich für eine Fachwechsel anmelden: 26 in der Deutschklasse Zone 1, und 21 in der Deutschklasse Zone 2. Nach einer zweiwöchigen Umstrukturierung am Semesteranfang sind insgesamt ca. 30 Studierende in die Englisch-Klasse umgeschrieben. Dabei kann die Mehrheit von Zone 2 wegen mangelnder Kapazität der Englischklassen nicht wechseln.

Tabelle 6: Gruppenstärken der Klassen vor und nach der Umstrukturierung

\begin{tabular}{lccl}
\hline Gruppenstärke & \multicolumn{1}{c}{$\begin{array}{l}\text { Am } \\
\text { Semesteranfang }\end{array}$} & $\begin{array}{l}\text { Nach der } \\
\text { Umstrukturierung }\end{array}$ & Fächer \\
\hline $\begin{array}{l}\text { Deutschklasse Zone 1 } \\
\text { (Gruppe A) }\end{array}$ & 60 & 36 & $\begin{array}{l}\text { Energiewissenschaft, } \\
\text { Maschinenbau, } \\
\end{array}$ \\
& & & $\begin{array}{l}\text { Umweltwissenschaft, } \\
\text { Chemieingenieurwesen, }\end{array}$ \\
& & Lebensmittelwissenschaft \\
Deutschklasse Zone 2 & 37 & 30 & Maschinenbau \\
(Gruppe B) & & Topographie \\
& & Bergbau \\
& & Geographie \\
& & Automatisierung \\
& & Bauingenieurwesen \\
& & Stadtplanung \\
\hline
\end{tabular}


Nach der Umstrukturierungsphase der ersten zwei Wochen dürfen die Studierenden die studienbegleitende Fremdsprache im Prinzip nicht mehr ändern. Sie bleiben in der Klasse für zwei Jahre bzw. 4 Semester. Jede Klasse hat 2 Doppelstunden ${ }^{17}$ pro Woche. Die Vorlesungszeit WS 2016/17 dauert insgesamt 16 Wochen, das heißt insgesamt 64 Unterrichtsstunden pro Semester für jede Klasse. Das Erlernen von Deutsch dauert zwei Studienjahre, insgesamt also 256 US.

In der dritten Woche wird eine Befragung zur Lernerbiographie sowie Lernerfahrung und Lernmotivation gegenüber der Fremdsprache durch elektronische Fragenbogen per Handy im Unterricht anonym und freiwillig durchgeführt. Das Ausfüllen der Befragung dauert 5 bis 10 Minuten.

Tabelle 7: Zahl der Anwesenden und der Beteiligten bei der Befragung zur Lernerbiographie

\begin{tabular}{lcc}
\hline & Anwesende Studierende & Teilnehmende Studierende \\
\hline Zone 1 (Gruppe A) & 36 & 34 \\
Zone 2 (Gruppe B) & 29 & 25 \\
\hline
\end{tabular}

Für die Studierenden, die wegen technischer oder anderer Gründe an der Befragung nicht teilgenommen haben, wird ein Link für die Nacharbeitung zur Verfügung gestellt, der für 7 Tage gültig ist. Allerdings hat keiner danach an der Befragung teilgenommen.

Die Befragung zur Lernbiographie (der Fragenbogen und dessen Ergebnisse siehe Anhang 1) bestehen aus folgenden Schwerpunkten:

Tabelle 8: Übersicht der Befragung zur Lernbiografie

\begin{tabular}{ll}
\hline Kriterien & $\begin{array}{l}\text { Operationalisiert in den Fragen } \\
\text { Nr. }\end{array}$ \\
\hline Persönliche Daten: Geschlecht, Alter und Herkunft & $1-3$ \\
Lernerbiographie: & $4-7$ \\
$\begin{array}{l}\text { Gelernte Fremdsprache, Lehrmethode usw. } \\
\text { Lernmotivation für Fremdsprachen im Allgemeinen }\end{array}$ & $8-11$ \\
$\begin{array}{l}\text { Schulzeitige Lernerfahrung } \\
\text { Lernmotivation fürs Studium und Deutsch }\end{array}$ & $12-18$ \\
\hline
\end{tabular}

Aus der Befragung kann man folgende Informationen von den Zielgruppen feststellen:

17 Eine DS beträgt 90 Minuten, besteht aus 2 US. 
Die Mehrheit der Probanden ist männlich: in der Gruppe A gibt es zwei Studentinnen und in der Gruppe B eine Studentin. Die Studierenden sind zwischen 17 und 20 Jahre alt und die meisten davon stammen aus der Provinz Yunnan. $53 \%$ aus Gruppe A und $64 \%$ aus Gruppe B stammen aus Städten und Regionen außerhalb der Hauptstadt Kunming, wo ungünstige fremdsprachliche Schulbedingungen herrschen (siehe Kapitel 4.1).

Alle Studierenden haben nur Englisch als Fremdsprache gelernt (vgl. Frage 4). Die Mehrheit hat 6 bis 10 Jahre Englisch gelernt: $82 \%$ der Gruppe A und $92 \%$ der Gruppe B.

Die Lernerfahrung beim Fremdsprachenlernen sieht nicht positiv aus. Viele interessieren sich für das Fremdsprachlernen nicht: 59 \% von der Gruppe A und $61 \%$ von der Gruppe B sind der Meinung, dass Fremdsprachenunterricht langweilig ist (vgl. Frage 8). Kommunikative Lern- und Lehrmethoden sind ihnen unbekannt. Die meisten erlebten Übungsformen im Fremdsprachenunterricht waren schriftliche Übungen für Grammatik, Wortschatz, Textschreiben und Diktat. Ein Student von Gruppe A hat einmal an einem Sprachlernspiel teilgenommen (vgl. Frage 7). Die Mehrheit ist der Meinung, dass ihre fremdsprachliche Lernerfahrung ihnen nicht geholfen hat, die Welt besser kennenzulernen oder die kommunikative Fähigkeit zu verbessern (vgl. Frage 10 und 11).

Die negative Lernerfahrung lässt verstehen, warum viele kein Selbstvertrauen beim Fremdsprachenlernen haben: 50 \% von der Gruppe A und $40 \%$ von der Gruppe B sind der Meinung, dass sie fremdsprachlich sehr unbegabt sind (vgl. Frage 5).

70 \% der Gruppe A und 80 \% der Gruppe B stimmen aber zu, dass das Erlernen einer Fremdsprache wichtig ist (vgl. Frage 9). Die Mehrheit bzw. 91 \% der Gruppe A und 92 \% der Gruppe B hat vor, im Studium ihre Fremdsprachenkenntnisse zu verbessern (vgl. Frage 12). Aber nur die Hälfte aller Befragten ist motiviert, den Wunsch zu realisieren: 56 \% von Gruppe A und 52 \% von Gruppe B glauben, dass sie Deutsch erlernen können (vgl. Frage 17). Bei der anderen Hälfte fehlt es an Selbstvertrauen: 47 \% der Gruppe A und 33 \% der Gruppe B empfinden die deutsche Sprache als zu schwierig, und 53 \% der Gruppe A und 50 \% der Gruppe B glauben, dass sie keine Begabung fürs Fremdsprachenlernen haben (vgl. Frage 18). Außerdem wählen nur wenige Lernende Deutsch, weil ihnen Deutsch gefällt oder weil sie nach Deutschland fahren und sich da weiterbilden möchten. Die meisten davon haben keine andere Wahl oder keine feste Vorstellung, welche Fremdsprache sie lernen möchten (vgl. Frage 13, 14 und 15).

Zusammenfassend lässt sich sagen, dass die Probanden für das Fremdsprachenlernen wenig motiviert sind. Sie interessieren sich kaum für die deutsche Sprache. Unbefriedigende Lernerfahrungen im Fremdsprachenunterricht 
führen dazu, dass sie sich für eine Fremdsprache allgemein wenig interessieren. Fehlendes Selbstvertrauen kann zur fehlenden WTC führen (siehe Kapitel 2.2.4), weswegen der Fremdsprachenunterricht der TUK nach Erfahrung der Autorin immer von der typischen Schweigsamkeit geprägt ist.

\subsubsection{Forschungsmaterialien: Lehrwerk, Spieladaption und Didaktisierung}

\section{Lehrwerk}

Die Studierenden arbeiten mit dem ersten Band des chinesischen Lehrwerks ” Klick auf Deutsch“ (Zhu und Li 2013). „Klick auf Deutsch“ ist eine vierbändige Lehrwerk-Reihe für Deutsch als studienbegleitendes Fach an chinesischen Universitäten, die im Lehrbücherplan des staatlichen elften Fünfjahresplans vorgesehen ist. Diese Lehrwerksammlung ist die neueste zum Hochschuldeutsch in China und berücksichtigt kommunikative Orientierung und Interkulturalität (vgl. Zhu 2007a, S. 149). Entwicklung und Konzept des Lehrwerkes werden im Beitrag von Zhu und Li (2004) ausführlich diskutiert, deshalb kann auf eine detaillierte Analyse in der vorliegenden Arbeit verzichtet werden.

Der erste Band besteht aus 9 themengebundenen Lerneinheiten und einer phonetischen Einführung. Die phonetische Einführung soll nach Lehrerhandbuch im Prinzip 8 US beanspruchen, jede andere Einheit 6 US. Die themengebundenen Lerneinheiten werden wie im Folgenden strukturiert (vgl. Zhu und Li 2011, S. 1):

Einführung: 10 Min.

Text und dazugehörige Übungen: 90 Min.

Grammatik und dazugehörige Übungen: 90 Min.

Intention: 25 Min.

Hörverstehen: 30 Min.

Leseverständnis: 25 Min.

In der Wiederholungsphase (13. bis 16. Semesterwoche) werden die Übungshefte zum Wortschatz und Leseverständnis der genannten Lehrwerk-Reihe benutzt. Manche Übungen von Texten, Grammatik, Hörverstehen und Leseverstehen in den genannten Lehrwerken und Übungsheften werden als Sprachlernspiele adaptiert (siehe Tabelle 9). 
Tabelle 9: Überblick über Gruppenverteilung und Lernmaterialien WS 2016-17

\begin{tabular}{|c|c|c|}
\hline Gruppe & $\begin{array}{c}\text { A } \\
\text { Kontrollgruppe, 36 P. }\end{array}$ & $\begin{array}{c}\text { B } \\
\text { Experimentalgruppe, 30 P. }\end{array}$ \\
\hline Lehrperson & \multicolumn{2}{|c|}{ ZENG Jing (die Forscherin) } \\
\hline Lehrmaterialien & \multicolumn{2}{|c|}{ Klick auf Deutsch: Lehrbuch, Übungsheft für Wortschatz, Übungsheft } \\
& & für Leseverstehen \\
\hline Übungsform & Übungen aus & Übungen aus genannten Lehrmaterialien \\
\cline { 3 - 3 } & genannten & adaptierte Übungen aus genannten \\
& Lehrmaterialien & Lehrmaterialien in der Form von \\
& & Sprachlernspielen (1 bis 3 Spiele pro \\
& & Doppelstunde, je nach Lerninhalt) \\
\hline
\end{tabular}

\section{Adaptionsbeispiele von Übungen ins Sprachlernspiel}

Um Chancengleichheit zu garantieren, benutzen die Kontrollgruppe (KG) und die Experimentalgruppe (EG) die gleichen Lernmaterialien. Die Übungen des ersten Bands „Klick auf Deutsch“ und dessen zugehörige Übungshefte sind nach bestimmten Formaten kategorisiert. Das bedeutet, dass Übungen mit ähnlichen Strukturen für bestimmte Fertigkeiten oder Kenntnisse in jedem Kapitel angeboten werden. Manche davon werden als Sprachlernspiele adaptiert und in der EG verwendet.

Hier zwei Beispiele von Übungen zur Grammatik und Wortschatz, um das Adaptionsverfahren zu veranschaulichen:

- Lückenfüllen-Übung als Kettenspiel

Das Kettenspiel ist eine spielerische Übungsform für Automatisierung und mündliches Training von sprachlichen Elementen. Im „Spiele im Deutschunterricht" (Dauvillier und Meese 2004, S. 27) wird ein Koffer-Packen-Spiel als Beispiel für Kettenspiel genannt:

Der erste Lernende beginnt: „in meine Tasche kommt: ein Heft. Und in deine?“

Der zweite Lernende fährt fort (mit oder ohne Wiederholung): „In meine kommt (ein Heft und) eine Landkarte: Und in deine..."

Eine im Lehrbuch „Klick auf Deutsch“ regelmäßig vorhandene Übung ist Lückenfüllen mit gegebenen Redemitteln, wie z. B. in der Grammatikübung (GÜ) 10 von Einheit 3 (Zhu und Li 2013, S. 65):

Redemittel: die Post, die Universität, der Börnerplatz, das Studentenwohnheim, das

Goethe-Haus, die Mensa

Beispiel: die Mensa

A: Kennst du die Mensa? 
B: Ja, kenne ich. Warum?

A: Ich suche sie.

Die Lernenden sollen nach dem Beispiel die richtige Form der gegebenen Redemittel in die Lücken füllen.

Dieser Übungstyp wird in folgenden Schritten als Kettenspiel adaptiert:

1. Vorbereitung:

$\rightarrow$ Zuerst soll die Gruppierung festgelegt werden. Da die EG aus 30 Lernenden besteht und es 6 vorgegebene Redemittel gibt, sollen 5 Gruppen gebildet werden.

$\rightarrow$ Die Lehrperson schreibt die Redemittel auf Karte: auf jede Karte ein Redemittel, 6 Karten pro Gruppe.

$\rightarrow$ Die Lehrperson bereitet ein Beispiel und die Redemittel in einer Powerpoint (PPT)- Präsentation vor, sodass es den Lernenden nicht bekannt ist, dass es sich um eine Übung aus dem Lehrwerk handelt.

2. Durchführung.

$\rightarrow$ Mit Hilfe der PPT- Präsentation wird das Beispiel sowie Mustersätze erklärt. Dann werden die Spielregeln ausführlich auf Chinesisch geklärt.

$\rightarrow$ Die Lernenden bilden 6er-Gruppen und stellen sich im Kreis auf. Jede Gruppe bekommt ein Set von Karten.

$\rightarrow$ Jeder Lernende soll nach der Information auf der Karte den Satz vor der Gruppe vollständig formulieren, einer spricht nach dem anderen wie beim Spiel Kofferpacken.

$\rightarrow$ Wenn sie mit einer Runde fertig sind, können sie die Karte wechseln und noch mal spielen. Die Lehrperson geht in dieser Phase im Klassenzimmer herum und gibt Vorschläge und Hilfe.

$\rightarrow$ Nach ca. 10 Min. sagt die Lehrperson, dass das Spiel zum Ende ist, und alle sollen ihren Platz einnehmen. Danach zeigt die Lehrperson, dass es sich um eine Übung auf Seit 65 im Lehrwerk handelt.

Die Spielform hat folgende Vorteile gegenüber der originalen Übung:

1. Die originale Übung ist eine schriftliche Einzelarbeit, während die adaptierte Version eine mündliche interaktive/kommunikative Gruppenarbeit ist. 
2. Die grammatikalischen Übungsziele bzw. konkret die Anwendung von Pronomen und bestimmten Artikeln im Akkusativ bleiben unverändert. Mit der Spielform können die Lernenden die genannten Sprachmittel auch mündlich üben, was die originale Übung nicht anbieten kann.

3. Die Spielform hilft bei der Binnendifferenzierung. Die Durchführung kann anhand individueller Unterschiede in jeder Gruppe anders organisiert werden. Fortgeschrittene können die Karte zudecken oder die zuvor gezeigten Karteninhalte wiederholen, wie im Bespiel der Kettenübung dargestellt wird, während schwächere Lernende mit Unterstützung der Karte die Redemittel üben können. Jede Gruppe kann im eigenen Tempo üben.

- Lückenfüllen-Übung von Verbkonjugationen als Kreuzworträtsel

Ein Kreuzworträtsel ist ein übliches Spiel, das Wissen bzw. Vokabeln zu bestimmten Themen testet. Es handelt sich normalerweise um ein Worträtsel oder eine Reihe von Fragen. Für die Fremdsprachenanfänger stellen viele Kreuzworträtsel eine Überforderung dar, weil man in der Lage sein muss, ein Worträtsel oder die gestellten Fragen in der Zielsprache zu verstehen und Wissen über einen anderen Kulturkreis zu beherrschen. Aber das Kreuzworträtsel muss kein Rätsel im allgemeinen Sinn sein.

Eine übliche Übungsform im Lehrwerk „Klick auf Deutsch“ ist die richtige konjugierte Form von Verben in die Lücken zu füllen, wie GÜ 4 aus Einheit 3 (ebenda, S. 64):

GÜ 4 Verbenspiel. Ergänzen Sie. schlafen

A: Wie viele Stunden du täglich?

B: Acht Stunden.

essen

A: Wann du zu Mittag?

B: Ich frühstücke meistens gut und nicht zu Mittag

Aus dieser Übung kann man ein Kreuzworträtsel adaptieren: 


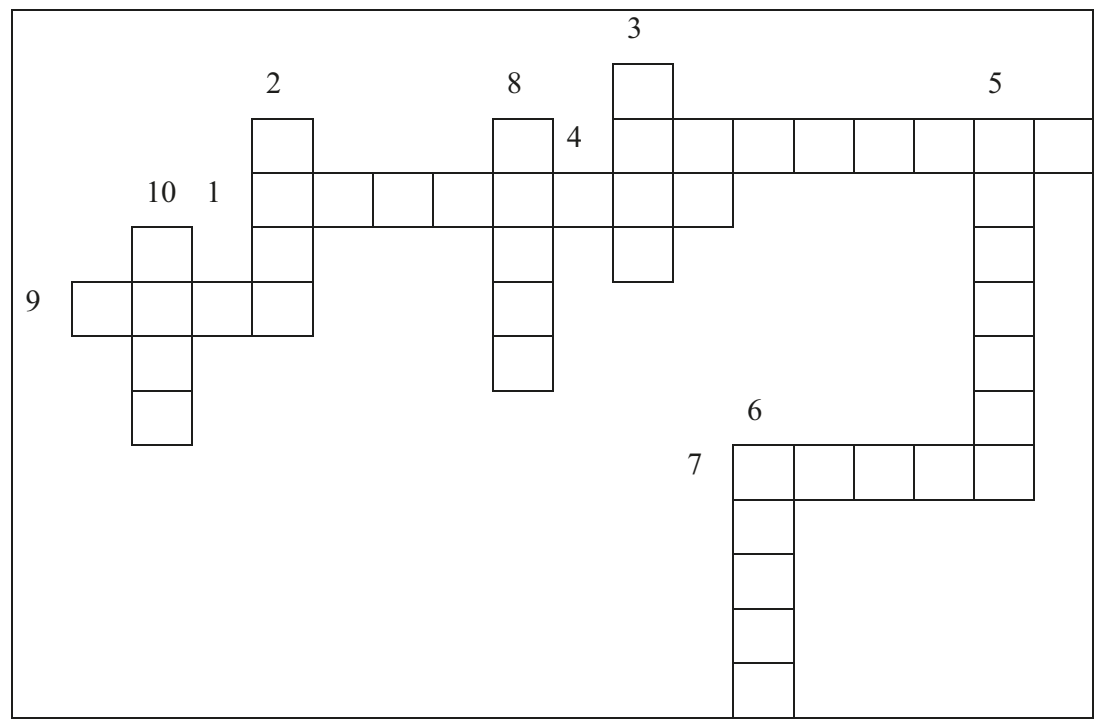

1. Wie viele Stunden (schlafen) du täglich?

2. Wann (essen) du zu Mittag?

3. Ich frühstücke meistens gut und (essen) nicht zu Mittag.

4. (sprechen) du Deutsch?

5. Die Frau (sprechen) auch Deutsch.

6. Ich (fahren) morgen nach München.

7. Wann (fahren) ihr?

8. Hans ( fahren) heute Nachmittag.

9. (haben) du heute Deutschunterricht?

10. Wann (haben) ihre Pause?

Die Lernenden arbeiten zuerst zu zweit mit dem Kreuzworträtsel. Die Lücken und deren Verbindungen helfen ihnen, die Antwort zu kontrollieren. Danach werden sie auf die Übung im Lehrwerk verwiesen und können mit Hilfe des Kreuzworträtsels die Übung weiter bearbeiten.

Das Kreuzworträtsel hat folgende Vorteile gegenüber der Übung im Lehrbuch:

1. Es handelt sich nicht mehr um eine Einzelarbeit, sondern um Partnerarbeit. Die Lernenden können zusammenarbeiten und sich gegenseitig helfen. 
2. Es ist interessanter und macht neugierig, weil ein Gewinnfaktor vorhanden ist.

3. Das Kreuzworträtsel dient auch als eine Möglichkeit für autonomes Lernen. Die Lernenden überprüfen die Antwort selbst mit Hilfe der Struktur und korrigieren sich selbst.

4. Auch der Wettbewerbsfaktor kann vorkommen, wenn z. B. die erste Gruppe, die das Rätsel richtig gelöst hat, einen kleinen Preis bekommen könnte.

\section{Die Didaktisierung}

Um die Störvariablen wie Vergesslichkeit und andere mögliche Probleme im Unterricht zu kontrollieren, wird ein Didaktisierungsplan vor jeder Stunde für beide Gruppen entworfen. Im Plan wird darauf hingewiesen, welche Übung in welcher Spielform adaptiert wird.

Mit dieser Didaktisierung kann die Forscherin die Unterrichtphasen dokumentieren, um die zeitliche Effizienz des Spiels und der Übung zu vergleichen.

Ein Beispiel der Didaktisierung von US 6 (45 Min.) Einheit 3 sieht wie folgt aus. Der mit ※ gekennzeichnete Teil ist das Spiel und die gekoppelte Übung.

Einheit 3 US 6 8. Woche 31.10. 2016 Gruppe B (EG)

Einführung 5 Min.

\begin{tabular}{|l|l|l|l|l|l|}
\hline Lernziele & Lernaktivitäten & Sozialform & Medien & Materialien & Lehraktivitäten \\
\hline $\begin{array}{l}\text { Vorwissen } \\
\text { aktivieren }\end{array}$ & TÜ 3 machen & Einzelarbeit & $\begin{array}{l}\text { PPT- } \\
\text { Präsentation }\end{array}$ & Lehrbuch & $\begin{array}{l}\text { Arbeitsanweisung } \\
\text { geben, erklären } \\
\text { und ermutigen }\end{array}$ \\
\hline
\end{tabular}

Präsentation 5 Min.

\begin{tabular}{|l|l|l|l|l|l|}
\hline Lernziele & $\begin{array}{l}\text { Lernaktivi- } \\
\text { täten }\end{array}$ & Sozialform & Medien & Materialien & Lehraktivitäten \\
\hline $\begin{array}{l}\text { Konjugation von } \\
\text { unregelmäßigen } \\
\begin{array}{l}\text { Verben im } \\
\text { Präsens kennen }\end{array}\end{array}$ & $\begin{array}{l}\text { Folien lesen } \\
\text { zuhören }\end{array}$ & Einzelarbeit & $\begin{array}{l}\text { PPT-Prä- } \\
\text { sentation }\end{array}$ & Lehrbuch & $\begin{array}{l}\text { Folien erklären } \\
\text { Hilfe geben, } \\
\text { erklären und } \\
\text { ermutigen }\end{array}$ \\
\hline
\end{tabular}


Semantisierung 15 Min.

\begin{tabular}{|l|l|l|l|l|l|}
\hline Lernziele & $\begin{array}{l}\text { Lernaktivi- } \\
\text { täten }\end{array}$ & Sozialform & Medien & Materialien & Lehraktivitäten \\
\hline $\begin{array}{l}\text { Konjugation der } \\
\text { unregelmäßigen } \\
\text { Verben im } \\
\text { Präsens lernen }\end{array}$ & $\begin{array}{l}\text { Folien lesen } \\
\text { Regel finden }\end{array}$ & Einzelarbeit & $\begin{array}{l}\text { PPT-Prä- } \\
\text { sentation }\end{array}$ & Lehrbuch & $\begin{array}{l}\text { Arbeitsanwei- } \\
\text { sung geben, } \\
\text { erklären Hilfe } \\
\text { geben, erklären } \\
\text { und ermutigen }\end{array}$ \\
\hline
\end{tabular}

Übung 20 Min.

\begin{tabular}{|c|c|c|c|c|c|}
\hline Lernziele & Lernaktivitäten & Sozialform & Medien & Materialien & Lehraktivitäten \\
\hline \multirow[t]{3}{*}{$\begin{array}{l}\text { Konjugation } \\
\text { der unregelmä- } \\
\text { ßigen Verben } \\
\text { im Präsens } \\
\text { anwenden } \\
\text { können }\end{array}$} & $\begin{array}{l}\text { Kreuzworträtsel } \\
\text { adaptiert aus } \\
\text { GÜ } 4 \text { machen ※ }\end{array}$ & $\begin{array}{l}\text { Partner- } \\
\text { arbeit }\end{array}$ & $\begin{array}{l}\text { PPT-Prä- } \\
\text { sentation }\end{array}$ & Lehrbuch & $\begin{array}{l}\text { Kreuzworträtsel } \\
\text { vorbereiten, ver- } \\
\text { teilen, Arbeits- } \\
\text { anweisung geben, } \\
\text { erklären und } \\
\text { helfen Arbeitsan- } \\
\text { weisung geben }\end{array}$ \\
\hline & & & & Spielkopie & $\begin{array}{l}\text { Zettel vorberei- } \\
\text { ten, verteilen } \\
\text { erklären und } \\
\text { helfen }\end{array}$ \\
\hline & $\begin{array}{l}\text { Spiel adaptiert } \\
\text { aus GÜ } 5 \\
\text { machen } ※\end{array}$ & $\begin{array}{l}\text { Partner- } \\
\text { arbeit }\end{array}$ & & & \\
\hline
\end{tabular}

Hausaufgabe GÜ 6

Einheit 3 US 6 8. Woche 31.10. 2016 Gruppe A (KG)

Einführung 5 Min.

\begin{tabular}{|l|l|l|l|l|l|}
\hline Lernziele & Lernaktivitäten & Sozialform & Medien & Materialien & Lehraktivitäten \\
\hline $\begin{array}{l}\text { Vorwissen } \\
\text { aktivieren }\end{array}$ & TÜ 3 machen & Einzelarbeit & $\begin{array}{l}\text { PPT- } \\
\text { Präsen- } \\
\text { tation }\end{array}$ & Lehrbuch & $\begin{array}{l}\text { Arbeitsanwei- } \\
\text { sung geben, } \\
\text { erklären } \\
\text { Hilfe geben, } \\
\text { erklären und } \\
\text { ermutigen }\end{array}$ \\
\hline
\end{tabular}


Präsentation 5 Min.

\begin{tabular}{|l|l|l|l|l|l|}
\hline Lernziele & $\begin{array}{l}\text { Lernaktivi- } \\
\text { täten }\end{array}$ & Sozialform & Medien & Materialien & Lehraktivitäten \\
\hline $\begin{array}{l}\text { Konjugation } \\
\text { von unregel- } \\
\text { mäßigen } \\
\text { Verben im } \\
\text { Präsens ken- } \\
\text { nen }\end{array}$ & zuhören & Einzelarbeit & $\begin{array}{l}\text { PPT-Prä- } \\
\text { sentation }\end{array}$ & Lehrbuch & $\begin{array}{l}\text { Arbeitsanweisung } \\
\text { geben und erklären } \\
\text { Hilfe geben, erklä- } \\
\text { ren und ermutigen }\end{array}$ \\
\hline
\end{tabular}

Semantisierung 15 Min.

\begin{tabular}{|c|c|c|c|c|c|}
\hline Lernziele & $\begin{array}{l}\text { Lernaktivi- } \\
\text { täten }\end{array}$ & Sozialform & Medien & Materialien & Lehraktivitäten \\
\hline $\begin{array}{l}\text { Konjugation } \\
\text { der unregel- } \\
\text { mäßigen } \\
\text { Verben im } \\
\text { Präsens lernen }\end{array}$ & $\begin{array}{l}\text { Folien lesen } \\
\text { Regel finden }\end{array}$ & Einzelarbeit & $\begin{array}{l}\text { PPT-Prä- } \\
\text { sentation }\end{array}$ & Lehrbuch & $\begin{array}{l}\text { Arbeitsanwei- } \\
\text { sung geben und } \\
\text { erklären } \\
\text { Hilfe geben, } \\
\text { erklären und } \\
\text { ermutigen }\end{array}$ \\
\hline
\end{tabular}

Übung 20 Min.

\begin{tabular}{|c|c|c|c|c|c|}
\hline Lernziele & Lernaktivitäten & Sozialform & Medien & Materialien & Lehraktivitäten \\
\hline \multirow{2}{*}{$\begin{array}{l}\text { Konjugation der } \\
\text { unregelmäßigen } \\
\text { Verben im Prä- } \\
\text { sens anwenden } \\
\text { können }\end{array}$} & GÜ 4 machen $※$ & \multirow{2}{*}{$\begin{array}{l}\text { Einzelarbeit } \\
\text { Plenum }\end{array}$} & \multirow{2}{*}{$\begin{array}{l}\text { PPT-Prä- } \\
\text { sentation }\end{array}$} & \multirow[t]{2}{*}{ Lehrbuch } & \multirow{2}{*}{$\begin{array}{l}\text { Arbeitsanwei- } \\
\text { sungen geben } \\
\text { erklären und } \\
\text { helfen }\end{array}$} \\
\hline & GÜ 5 machen ※ & & & & \\
\hline
\end{tabular}

Hausaufgabe GÜ 



\section{Empirische Untersuchung Teil I: Die Wirkung von Sprachlernspielen auf Motivation und Lernfortschritt von DaF- Lernenden in China}

\subsection{Forschungsphase Wintersemester 2016-17}

\subsubsection{Forschungsdesign Wintersemester 2016-17}

Die experimentelle Phase der Forschung beginnt erst in der fünften Semesterwoche nach der dreiwöchigen Einführung von Phonetik (Einheit 1 vom Lehrwerk), weil es in den ersten zwei Wochen eine Umstrukturierung des Stundenplans der Lernenden gibt und die Lehrveranstaltungen in der vierten Woche wegen nationaler Feiertage ausfällt. Im WS 2016-2017 wird das Erlernen von vier Einheiten ${ }^{18}$ und eine dreiwöchige Wiederholungsphase am Ende des Semesters geplant (siehe Abbildung 9).

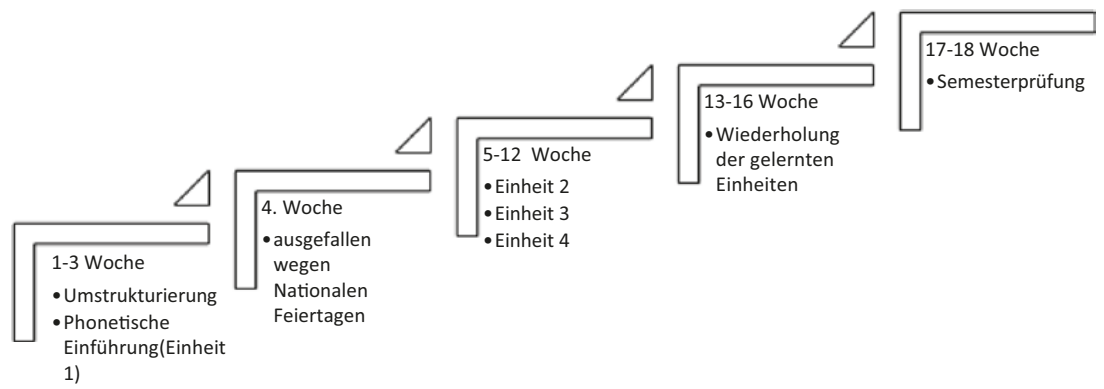

Abbildung 9: Überblick des Plans WS 2016-17

Während des Semesters werden verschiedene Datenerhebungsmethoden verwendet, die in der Abbildung 10 dargestellt werden.

18 Wegen der Bildungsdiskrepanz kann die Einheit nicht in dem im Lehrerhandbuch empfohlenen Tempo (siehe Kapitel 4.2.3) durchgeführt werden. 


\begin{tabular}{|c|c|c|c|}
\hline \multicolumn{4}{|c|}{ Lehrtagebuchhaltung von jeder Stunde ab des Experiments } \\
\hline \multirow{4}{*}{\begin{tabular}{|l|} 
zeitlicher Verlauf des \\
Unterrichts \\
Probleme der \\
Spielorganisation \\
Vergleich zweier Gruppen \\
Reflexion
\end{tabular}} & \multicolumn{3}{|c|}{ Befragung zur Lernbiographie am Semesterbeginn } \\
\hline & \multirow{3}{*}{$\begin{array}{l}\text { Persönliche Daten: } \\
\text { Geschlecht, Alter und } \\
\text { Herkunft } \\
\text { Lernmotivation für } \\
\text { Fremdsprachen im } \\
\text { Allgemeinen } \\
\text { fremdsprachliche } \\
\text { Lernerfahrung } \\
\text { Lernmotivation fürs } \\
\text { Studium und Deutsch }\end{array}$} & \multicolumn{2}{|c|}{ Befragungen am Semesterende } \\
\hline & & & Semesterprüfung \\
\hline & & $\begin{array}{l}\text { Einstellung gegenüber } \\
\text { Deutsch } \\
\text { Bewertung des } \\
\text { Unterrichts } \\
\text { Bewertungen zur } \\
\text { Methode } \\
\text { Sprachlernspiele (nur EG) }\end{array}$ & $\begin{array}{l}\text { sprachliche Kompetenzen } \\
\text { von Hören, Lesen, } \\
\text { Übersetzen, Schreiben } \\
\text { und Grammatik sowie } \\
\text { Wortschatz }\end{array}$ \\
\hline
\end{tabular}

Abbildung 10: Übersicht der Datenerhebungsmethoden im WS 2016-17

Die Befragungen am Semesterende bestehen aus zwei unabhängigen Teilen:

1. Befragung zur Motivation und Unterrichtsbewertung für beide Gruppen (siehe Anhang 2)

2. Befragung zur Einstellung und Bewertung gegenüber Sprachlernspielen für nur EG (siehe Anhang 3)

In der Befragung zur Motivation und Unterrichtsbewertung werden Items zu den folgenden Kriterien gegeben:

Tabelle 10: Kriterien und Itemüberblick der Befragung zur Lernmotivation und Unterrichtsevaluation WS 2016-17

\section{Kriterien}

Einstellung gegenüber Fremdsprache bzw. Deutsch

Selbstvertrauen bezüglich Fremdsprachenlernen

Investierte Lernzeit außerhalb des Unterrichts

Motivation im Unterricht beim Deutschsprechen

Interaktivität im Unterricht

Wissen zur interkulturellen Kommunikation

Evaluation des Unterrichts und des Unterrichtsplans
Operationalisiert in den Items $\mathrm{Nr}$.

$$
\begin{gathered}
1(1.1-1.6), 3 \\
2 \\
4 \\
5.1,5.2 \\
5.3-5.6,6 \\
7(7.1-7.3) \\
8(8.1-8-8), 9,10
\end{gathered}
$$


Ziel der Befragung ist, potenzielle Unterschiede von Motivation, Interaktion und Unterrichtsevaluation zwischen zwei Gruppen herauszufinden.

Eine Befragung zum Einsatz der Sprachlernspiele für die EG wird am Ende des Semesters durchgeführt, um die Bewertung sowie Meinungen über den Einsatz von Sprachlernspielen von Lernenden zu sammeln. Diese Befragung besteht aus folgenden Kriterien:

Tabelle 11: Kriterien und Itemüberblick der Befragung zum Einsatz von Sprachlernspielen WS 2016-17

Kriterien

Operationalisiert in

den Items Nr.

Über den Einsatz von Sprachlernspielen im Unterricht allgemein

1,2

Über die Funktionen von Sprachlernspielen im Unterricht

Über Einzelheiten der eingesetzten Spiele in dem Semester

$4(4.1-4.9)$

Über bevorzugte Spieltypen sowie zur Verfügung stehende Zeit

5,6

Stellungnahme zum Unterricht mit oder ohne Sprachlernspiel

$7,8,9,10$

Die Ergebnisse dieser Befragung sollen Einzelheiten zum Spieleinsatz im Unterricht liefern. Damit soll herausgefunden werden, ob und ggf. wie die Spiele in den Augen der Studierenden funktionieren, welchen Spieltyp sie bevorzugen und ob sie spielen möchten, wenn ja, wie lange und wie überhaupt.

Der potenzielle Unterschied beim Lernfortschritt wird durch die Leistungen der Semesterprüfung überprüft.

\subsubsection{Auswertung und Analyse zu der Befragung zur Motivation und Unterrichtsevaluation im Wintersemester 2016-17}

Die schriftliche Befragung zur Motivation und Unterrichtsevaluation (siehe Anhang 2) wird in der 14. Woche am 12. Dezember 2016 im Unterricht durchgeführt. Die Anonymität der Befragung wird bekannt gemacht. Die Studierenden wissen, dass die Befragung auf ihre Benotung keinen Einfluss ausüben kann. Die Befragung wird durch einen Pretest von 4 Personen mehrmals überprüft und verbessert, um sicherzustellen, dass die Fragen verständlich wirken und die Zeit des Beantwortens nicht länger als 15 Min. ist. Die Befragung ist in der Muttersprache der Lernenden (Chinesisch) verfasst.

Insgesamt sind 56 beantwortete Fragenbogen in die Auswertung einbezogen. Darunter 26 von der EG, 30 von der KG. 
Folgenden Hypothesen werden aufgestellt:

1. Die EG ist mehr motiviert als die KG.

2. Die EG gibt dem Unterricht eine bessere Bewertung als die KG.

3. Der Unterricht der EG wird interaktiver als der von der KG bewertet.

Um zu überprüfen, ob zwei zu vergleichende Stichproben signifikante unterschiedliche Messewerte zeigen, kann man den „t-Test“ benutzen. Die Voraussetzung für die Anwendbarkeit des „t-Tests“ ist die Normalverteilung der Variablen (vgl.Settinieri 2012, S. 263). Die Ergebnisse des Kolmogorov-Smirnov Tests durch SPSS (siehe Anhang 5) zeigen aber, dass die Befragungsergebnisse keiner Normalverteilung entsprechen, $\mathrm{D}(\mathrm{KG})=30, \mathrm{p}<0.05^{19}$; $\mathrm{D}(\mathrm{EG})=26, \mathrm{P}<0.05$. In diesem Fall kann man den Mann-Whitney-U-Test (MWU-Test) benutzen, um signifikante unterschiedliche Messwerte zu überprüfen (vgl. Settinieri 2012, S. 262; Qin und Bi 2016, S. 309). Um ein einheitliches statistisches Verfahren des Projekts zu sichern, werden alle Daten im gesamten Projekt mit MWU-Test durch SPSS analysiert.

Aus Ergebnissen der MWU-Tests (siehe Anhang 6) wird ersichtlich, dass nur bei Item 1.3, 5.5, 6 und 8.6 signifikante Unterschiede zwischen beiden Gruppen festgestellt werden können. Im Anschluss daran sollen diese 4 Items jetzt unter die Lupe genommen werden.

Tabelle 12: Überblick der Items mit signifikantem Unterschied von der Befragung zur Motivation und Unterrichtsevaluation WS 2016-17

\begin{tabular}{|c|c|c|c|c|c|c|c|}
\hline \multirow[t]{2}{*}{$\overline{\text { Item }}$} & \multicolumn{2}{|c|}{ Mittelwert } & \multirow{2}{*}{$\begin{array}{c}\text { Mann- } \\
\text { Whitney-U }\end{array}$} & \multirow[t]{2}{*}{ Wilcoxon W } & \multirow[t]{2}{*}{$\mathbf{Z}$} & \multirow{2}{*}{$\begin{array}{l}\text { Asymp. Sig. } \\
\text { (zweiteilig) }\end{array}$} & \multirow{2}{*}{$\begin{array}{c}\text { P. } \\
\text { (= Sig./ } 2\end{array}$} \\
\hline & EG(26) & KG(30) & & & & & \\
\hline 1.3 & 3.0769 & 2.3000 & 261 & 726 & -2.224 & 0.026 & $0.013^{\star}$ \\
\hline 5.5 & 3.5769 & 3.0333 & 278.5 & 743.5 & -1.889 & 0.059 & $0.0295^{*}$ \\
\hline 6 & 4.5769 & 3.7667 & 152.5 & 617.5 & -4.223 & 0 & $0^{*}$ \\
\hline 8.6 & 2.5385 & 2.9000 & 272.5 & 623.5 & -2.361 & 0.018 & $0.009^{*}$ \\
\hline
\end{tabular}

Zu Item 1.3 „Wenn ich kann, will ich lieber Englisch lernen. (1 ich stimme gar nicht zu - 5 ich stimme völlig zu) “ ist die einteilige Signifikanz $\mathrm{p}=0.013<0.05$ zu sehen. Damit kann man feststellen, dass die EG Englisch signifikant mehr den

19 In der Fremdsprachenforschung wird in der Mehrzahl der Studien das folgende Signifikanzniveau $(P)$ zugrunde gelegt: $p>0.05$ nicht signifikant, $p \leq 0.05$ siginifikant, $p \leq 0.01$ sehr siginifikant, $\mathrm{p} \leq 0.001$ hoch signifikant (vgl. Settinieri 2012, S. 260f.). In der vorliegenden Arbeit wird die signifikante Zahl bzw. höhere Zahl mit * gekennzeichnet. 
Vorzug vor Deutsch gibt als die KG. Das lässt sich aus der Tatsache herleiten, dass 13 Studierende der EG Englisch lernen wollten, aber wegen organisatorischer Gründe das Fach nicht wechseln konnten.

Zur Interaktion im Unterricht zeigen die zwei Gruppen einen deutlichen Unterschied: Item 5.5 „Im Deutschunterricht mache ich die Aufgaben meistens mit meinen Kommilitonen zusammen... (1 ich stimme gar nicht $\mathrm{zu}-5 \mathrm{ich}$ stimme völlig $\mathrm{zu}$ ) “ zeigt einen signifikanten Unterschied: $\mathrm{p} \approx 0.03<0.05$. Damit wird die höhere Interaktivität von der EG bestätigt. Dieser Unterschied wird vom Item 6 noch deutlicher hervorgehoben. Unter Item 6 „Bitte bewerten Sie die Interaktivität im Unterricht im Allgemein (1 sehr schwach - 5 sehr stark)“ findet man hoch signifikanten Unterschied, $\mathrm{p}=0.00 \leq 0.001$. Das heißt, dass der Unterricht von den Lernenden der EG deutlich interaktiver als von den Lernenden der KG bewertet wird.

Der hochsignifikante Unterschied $(\mathrm{p}=0.009<0.01)$ bei Item 8.6 „Bitte bewerten Sie, ob folgende Faktoren im Unterricht zeitlich richtig behandelt werden (1. zuwenig Zeit - 5 zu viel Zeit): Übungen zum Leseverständnis“ beweist, dass die Lernenden der EG der Meinung sind, dass im Unterricht mehr Zeit dem Leseverständnis gewidmet werden soll, während die Lernenden der KG die Zeit für angemessen halten. Das lässt vermuten, dass die EG wegen der Sprachlernspiele die Lesenverständnis-Übungen nicht wie gewöhnlich wahrnehmen.

Außer den genannten statistisch signifikanten Unterschieden zur Unterrichtsinteraktion und dem Vorziehen der Sprache des Lernenden, soll auch die Selbsteinschätzung der Sprachlernfähigkeit unter die Lupe genommen werden, um die potenzielle Änderung während des Semesters zu erkennen.

Zur Item 2 „Bitte bewerten Sie Ihre Sprachlernfähigkeit im Allgemein. (1 sehr schlecht - 5 sehr gut)“ gibt die EG leicht höhere Selbsteinschätzungsnoten in den Rohdaten an. Die einteilige Signifikanz $p=0.252 / 2=0.126>0.05$ deutet allerdings darauf hin, dass die EG keine statistisch signifikant bessere Bewertung der eigenen Sprachlernfähigkeit als die KG angibt.

Tabelle 13: Ergebnisse vom MWU-Test von Item 2 der Befragung zur Motivation und Unterrichtsevaluation WS 2016-17

\begin{tabular}{cccccccc}
\hline Item & \multicolumn{2}{c}{ Mittelwert } & \begin{tabular}{c} 
Mann- \\
\cline { 2 - 3 } Whitney-U
\end{tabular} & Wilcoxon W & Z & $\begin{array}{c}\text { Asymp. Sig. } \\
\text { (zweiteilig) }\end{array}$ & $\begin{array}{c}\text { P. } \\
(=\text { Sig./ 2) }\end{array}$ \\
\hline 2 & 2.5385 & 2.2000 & 324.5 & 789.5 & -1.146 & 0.252 & 0.126 \\
\hline
\end{tabular}


Im Vergleich zur Befragung am Semesteranfang zur Lernbiographie kann man aber eine Steigerung in beiden Gruppen beobachten: EG $+0.63, \mathrm{KG}+0.31$. Werden die Daten mit dem MWU Test überprüft, kann festgestellt werden, dass die Selbstbewertung am Anfang und am Ende des Semesters bei der KG keinen signifikanten Unterschied zeigt: $\mathrm{p}=0.073>0.05$, während man einen signifikanten Unterschied bei der EG feststellen kann: $\mathrm{p}=0.016<0.05$ :

Tabelle 14: Ergebnisse vom MWU-Test von Item 2 am Anfang und Ende des Semesters WS 2016-17

\begin{tabular}{lcccccccc}
\hline Gruppe & $\begin{array}{c}\text { Mittel- } \\
\text { wert } \\
\begin{array}{c}\text { Semes- } \\
\text { teran- } \\
\text { fang }\end{array}\end{array}$ & $\begin{array}{c}\text { Mittel- } \\
\text { wert } \\
\text { Semes- } \\
\text { terende }\end{array}$ & $\begin{array}{c}\text { Steige- } \\
\text { rung der Whitney-U } \\
\text { Bewer- } \\
\text { tung }\end{array}$ & & & $\begin{array}{c}\text { Mann- } \\
\text { W }\end{array}$ & $\begin{array}{c}\text { Wilcoxon } \\
\text { Sig. } \\
\text { (zweitei- } \\
\text { lig) }\end{array}$ & $\begin{array}{c}\text { P. } \\
\text { (= Sig./ } \\
\text { 2) }\end{array}$ \\
\hline EG & 1.91 & 2.54 & +0.63 & 197.000 & 473.000 & -2.140 & .032 & $0.016^{*}$ \\
KG & 1.89 & 2.2 & +0.31 & 408.500 & 1003.500 & -1.454 & .146 & 0.073 \\
\hline
\end{tabular}

Die statistischen Ergebnisse zeigen, dass die Studierenden der EG ihre eigenen Sprachlernkompetenzen am Semesterende deutlich höher als am Semesteranfang einschätzen. Auf Basis der Daten scheint es naheliegend, dass das Selbstvertrauen bezüglich des Fremdsprachenlernens der EG im WS 2016-17 deutlich verbessert wird, während das der KG keine signifikante Änderung zeigt.

Aus der statistischen Auswertung wird geschlossen, dass die Hypothese 1 „Die EG ist mehr motiviert als die KG. "bestätigt werden kann. Während beide Gruppen keinen statistisch signifikanten Unterschied zum Stellenwert der Zielsprache zeigen, erreicht die EG mit Sprachlernspielen eine signifikant erhöhte Selbsteinschätzung ihrer Sprachlernkompetenz als die KG ohne Sprachlernspiele.

Die Hypothese 3 „Der Unterricht der EG wird interaktiver als der von der KG bewertet. "wird durch das hohe Signifikanzniveau von Item 5.5 und 6 bestätigt.

Allerdings verspricht die höhere Interaktivität keine höhere Bewertung des Unterrichts. Bei der Unterrichtsbewertung von Item 9 zeigen die Ergebnisse beider Gruppen keinen signifikanten Unterschied: $\mathrm{P}=0.464 / 2=0.232>0.05$ (siehe Tabelle 15). Die Hypothese 2 „Die EG gibt dem Unterricht eine bessere Bewertung als die $K G$ “ muss widergelegt werden. 
Tabelle 15: Ergebnisse vom MWU-Test von Item 9 im WS 2016-17

\begin{tabular}{|c|c|c|c|c|c|c|c|}
\hline \multirow{2}{*}{$\begin{array}{c}\text { Item } 9 \\
\text { Bitte bewerten Sie } \\
\text { Deutschunterricht } \\
\text { im Allgemein. (1 sehr } \\
\text { schlecht-5 sehr gut) } \\
\end{array}$} & \multicolumn{2}{|c|}{ Mittelwert } & \multirow{2}{*}{$\begin{array}{c}\text { Mann- } \\
\text { Whitney- } \\
-\quad \text { U }\end{array}$} & \multirow[t]{2}{*}{$\begin{array}{c}\text { Wilcoxon } \\
\text { W }\end{array}$} & \multirow[t]{2}{*}{$\mathrm{Z}$} & \multirow[t]{2}{*}{$\begin{array}{l}\text { Asymp. Sig. } \\
\text { (zweiteilig) }\end{array}$} & \multirow{2}{*}{$\begin{array}{c}\text { P. }(= \\
\text { Exact } \\
\text { Sig./ 2) }\end{array}$} \\
\hline & $\begin{array}{l}\text { EG } \\
(26)\end{array}$ & $\begin{array}{l}\text { KG } \\
(30)\end{array}$ & & & & & \\
\hline & 4.15 & 4.03 & 350.000 & 815.000 & -.732 & .464 & $\begin{array}{l}0.232 \\
>0.05\end{array}$ \\
\hline
\end{tabular}

Zusammenfassend lässt sich sagen, dass der Vermittlungsweg mit Sprachlernspielen zu keinem signifikanten Unterschied bei der Unterrichtsevaluation führt, aber die Interaktion im Unterricht erhöht und die Motivation der Lernenden teilweise verbessern kann.

\subsubsection{Auswertung und Analyse zu den Ergebnissen der Semesterprüfungen im Wintersemester 2016-17}

Die Englisch-Einstufungstestergebnisse ${ }^{20}$ am Anfang des Semesters werden überprüft, um feststellen zu können, ob die zwei Gruppen einen signifikanten Unterschied in der Englisch-Leistung zeigen.

Tabelle 16: Ergebnisse vom MWU-Test des Englischeinstufungstests

\begin{tabular}{|c|c|c|c|c|c|c|c|}
\hline \multirow{2}{*}{$\begin{array}{l}\text { Englisch- } \\
\text { Einstufungs- } \\
\text { test }\end{array}$} & \multicolumn{2}{|c|}{ Mittelwert } & \multirow{2}{*}{$\begin{array}{l}\text { Mann- } \\
\text {-Whitney- } \\
\text { U }\end{array}$} & \multirow{2}{*}{$\begin{array}{l}\text { Wilcoxon } \\
\text { W }\end{array}$} & \multirow[t]{2}{*}{$\bar{Z}$} & \multirow{2}{*}{$\begin{array}{l}\text { Asymp. Sig. } \\
(\text { zweiteilig })\end{array}$} & \multirow{2}{*}{$\begin{array}{l}\text { P. } \\
\text { (= Asymp. } \\
\text { Sig../ 2) }\end{array}$} \\
\hline & $\begin{array}{l}\text { EG } \\
(30)\end{array}$ & $\begin{array}{l}\text { KG } \\
(36)\end{array}$ & & & & & \\
\hline & 33.17 & 30.85 & 451.500 & 1117.500 & -1.141 & .254 & $\begin{array}{l}0.127 \\
>0.05\end{array}$ \\
\hline
\end{tabular}

Das Ergebnis lässt keinen signifikanten Unterschied der Englischprüfungsnoten zwischen den zwei Gruppen erkennen $(P=0.127>0.05)$. Das bedeutet, dass die beiden Gruppen am Anfang des Semesters gleiches fremdsprachliches Niveau und gleiche Lernfähigkeit zeigen.

20 Rohdaten siehen Anhang 7: Notenübersicht der Semesterprüfung Wintersemester 2016-17 sowie Einstufungstestnote und Abiturnote vom Englisch 
Nach der 16-wöchigen Vorlesungszeit nehmen die Lernenden in der 18. Woche des WS 2016-17 an der Semesterprüfung für Deutsch teil. Die Prüfung besteht aus zwei Klausuren:

Klausur 1: Test für Hörverstehen, Übersetzen und Schreiben;

Klausur 2: Test für Grammatik, Wortschatz und Leseverstehen.

Jeder Test ermöglicht insgesamt 100 Punkte, 60 Punkte heißt bestanden. Die Notenverteilung zu jeder Teilfertigkeit ist wie folgt dargestellt:

Tabelle 17: Übersicht der Notenverteilung der Semesterprüfung WS 2016-17

\begin{tabular}{lccc}
\hline Klausur 1 & $\begin{array}{c}\text { Gesamtpunkt } \\
\text { Teilfertigkeiten }\end{array}$ & Klausur 2 & $\begin{array}{c}\text { Gesamtpunkt } \\
\text { Teilfertigkeiten }\end{array}$ \\
\hline Hörverstehen & 50 & Grammatik und & 75 \\
Übersetzen & 40 & Wortschatz & \\
Schreiben & 10 & Leseverstehen & 25 \\
\hline
\end{tabular}

Die Prüfung findet am 9. Jan. 2017 in einem Hörsaal statt, in dem die EG und die KG die Prüfung zusammen absolvieren. 2 Aufsichtslehrende sind für die Verteilung und das Einsammeln der Prüfungsbögen sowie die Beaufsichtigung der Prüfung verantwortlich. Die gesamte Prüfung dauert 150 Min. Die Prüfungsteilnehmer bekommen erst die Prüfungsbögen von Klausur 1. Wenn sie mit der Klausur 1 fertig sind, melden sie sich, um die Prüfungsbögen der Klausur 1 abzugeben und die der Klausur 2 zu bekommen.

Der Inhalt der Prüfung und die Struktur der Items entsprechen den gelernten Einheiten und den Übungen des WS 2016-17. Beide Gruppen haben den gleichen Inhalt gelernt. Studierende der EG haben manche Übungen spielerisch erarbeitet, während die der KG nach der Angabe des Lehrwerks geübt haben.

Insgesamt haben 30 Studierende aus der EG und 36 Studierende aus der KG an der Prüfung teilgenommen. Folgende Hypothese wird gestellt und soll überprüft werden:

Die EG mit Sprachlernspielen zeigt bessere Leistung als die KG ohne Sprachlernspiele.

Die Klausurergebnisse ${ }^{21}$ werden durch SPSS mit dem MWU- Test analysiert, um einen möglichen statistischen Unterschied in beiden Gruppen herauszufinden. Die MWU-Test-Ergebnisse sind wie folgt dargestellt:

21 Rohdaten von Prüfungsnoten siehe Anhang 7: Notenübersicht der Semesterprüfung Wintersemester 2016-17 sowie Einstufungstestnote und Abiturnote vom Englisch. 
Tabelle 18: Ergebnisse vom MWU-Test der Klausur 1

\begin{tabular}{llllllll}
\hline $\begin{array}{l}\text { Gesamtnote } \\
\text { Klausur 1 }\end{array}$ & \multicolumn{2}{c}{ Mittelwert } & $\begin{array}{c}\text { Mann- } \\
\text { Whitney- U }\end{array}$ & $\begin{array}{c}\text { Wilcoxon } \\
\text { W }\end{array}$ & $\begin{array}{c}\text { Asymp. Sig. } \\
\text { (zweiteilig) }\end{array}$ & $\begin{array}{c}\text { P. } \\
(=\text { Sig./ 2) }\end{array}$ \\
\cline { 2 - 7 } & $\begin{array}{l}\text { KG } \\
(\mathbf{3 0})\end{array}$ & $\begin{array}{l}\text { (36) } \\
(36\end{array}$ & & & & & \\
\hline & 62.48 & 64.20 & 527.000 & 1193.000 & -.167 & .867 & $0.434>0.05$ \\
\hline
\end{tabular}

Tabelle 19: Ergebnisse vom MWU-Test der Klausur 2

\begin{tabular}{|c|c|c|c|c|c|c|c|}
\hline \multirow{2}{*}{$\begin{array}{l}\text { Gesamtnote } \\
\text { Klausur } 2\end{array}$} & \multicolumn{2}{|c|}{ Mittelwert } & \multirow{2}{*}{$\begin{array}{c}\text { Mann- } \\
\text { Whitney- U }\end{array}$} & \multirow{2}{*}{$\begin{array}{c}\text { Wilcoxon } \\
\text { W }\end{array}$} & \multirow[t]{2}{*}{$\mathrm{Z}$} & \multirow{2}{*}{$\begin{array}{l}\text { Asymp. Sig. } \\
\text { (zweiteilig) }\end{array}$} & \multirow{2}{*}{$\begin{array}{c}\text { P. } \\
(=\text { Sig./ 2) }\end{array}$} \\
\hline & $\begin{array}{l}\text { EG } \\
(30)\end{array}$ & $\begin{array}{l}\text { KG } \\
(36)\end{array}$ & & & & & \\
\hline & 57.61 & 60.9 & 483.500 & 948.500 & -.728 & .467 & $0.234>0.05$ \\
\hline
\end{tabular}

Zusammenfassend lässt sich sagen, dass kein statistisch signifikanter Unterschied bei der Gesamtnote beider Klausuren festgestellt werden kann.

Wenn man die Teilkompetenzen beider Gruppen betrachtet, zeigt sich ein signifikanter Unterschied bei der Fertigkeit Schreiben: $\mathrm{P}=0.04<0.05$ (siehe Tabelle 20). Das heißt, dass die Studierenden der EG statistisch bessere Noten beim Schreiben als die der KG bekommen haben.

Tabelle 20: Ergebnisse vom MWU-Test der Teilfertigkeiten WS 2016-17

\begin{tabular}{|c|c|c|c|c|c|c|c|}
\hline \multirow{2}{*}{$\begin{array}{l}\text { Note der } \\
\text { Teilfertigkeiten }\end{array}$} & \multicolumn{2}{|c|}{ Mittelwert } & \multirow{2}{*}{$\begin{array}{c}\text { Mann- } \\
\text { Whitney- U }\end{array}$} & \multirow{2}{*}{$\begin{array}{c}\text { Wilcoxon } \\
\text { W }\end{array}$} & \multirow[t]{2}{*}{ Z } & \multirow{2}{*}{$\begin{array}{l}\text { Asymp. Sig. } \\
\text { (zweiteilig) }\end{array}$} & \multirow{2}{*}{$\begin{array}{c}\text { P. } \\
\text { (= Sig./ 2) }\end{array}$} \\
\hline & $\begin{array}{l}\text { EG } \\
(30)\end{array}$ & $\begin{array}{l}\text { KG } \\
(36)\end{array}$ & & & & & \\
\hline Hörverstehen & 32.33 & 32.9 & 519.000 & 984.000 & -.272 & .786 & $0.393>0.05$ \\
\hline Übersetzen & 24.15 & 25.89 & 527.000 & 992.000 & -.168 & .867 & $0.4335>0.05$ \\
\hline Schreiben ${ }^{*}$ & $6^{*}$ & 5.42 & 406.000 & 1072.000 & -1.751 & .080 & $0.04<0.05^{\star}$ \\
\hline $\begin{array}{l}\text { Grammatik und } \\
\text { Wortschatz }\end{array}$ & 37.8 & 40.83 & 474.500 & 939.500 & -.844 & .399 & $0.1995>0.05$ \\
\hline Leseverstehen & 19.8 & 20.07 & 507.500 & 972.500 & -.420 & .675 & $0.3375>0.05$ \\
\hline
\end{tabular}

Aufgrund der MWU-Testergebnisse der Semesterprüfung lässt sich sagen, dass die Vermittlungswege zu keinem signifikanten Unterschied in der Gesamtleistung führen. Die Hypothese „Die EG mit Sprachlernspielen zeigt bessere Leistung als die KG ohne Sprachlernspiel" wird nur bei der Teilfertigkeit Schreiben bestätigt. 
Mit Hilfe von SPSS kann man den Index der lexikalischen Vielfalt der Leistungen bekommen, der einen tieferen Blick auf die Leistung erlaubt und die Ergebnisse differenzierter betrachten lässt (vgl. Grum 2012, S. 274). Für jede Probandengruppe wird der jeweilige Leistungsmittelwert, der Median, die Standardabweichung und die Spannweite innerhalb der Gruppen gezeigt (siehe Tabelle 21).

Die Klausurnoten der EG weisen eine höhere Streuung auf als die der KG, was an den höheren Werten für die Standardabweichung und die Spannweite zu erkennen ist. Das heißt, dass die Heterogenität der Klausurnoten von der EG bezüglich der lexikalischen Vielfalt größer als die von der KG ist.

Auf der anderen Seite sieht man auch, dass die Standardabweichung der KG des Englischeinstufungstests höher als die der EG ist. Das heißt, dass die Heterogenität der EG am Anfang des Semesters kleiner als die der KG ist.

Tabelle 21: Übersicht des Index lexikalischer Vielfalt der Semesterprüfung WS 2016-17

\begin{tabular}{lcccccccc}
\hline Index & \multicolumn{1}{c}{ Mittelwert } & \multicolumn{2}{c}{ Median } & \multicolumn{3}{c}{ Standardabweichung } & \multicolumn{3}{c}{ Spannweite } \\
\hline Gruppe & EG & KG & EG & KG & EG & KG & EG & KG \\
& $(30)$ & $(36)$ & $(30)$ & $(36)$ & $(30)$ & $(36)$ & $(30)$ & $(36)$ \\
Gesamtnote Klausur 1 & 62.48 & 64.2 & 66 & 65 & $18.24^{*}$ & 12.55 & $64.5^{*}$ & 57 \\
Hörverstehen & 32.33 & 32.9 & 32 & 33.5 & 5.381 & $5.83^{*}$ & 20 & $26^{*}$ \\
Übersetzen & 24.15 & 25.89 & 26.75 & 29.5 & $12.14^{*}$ & 8.32 & $38.5^{*}$ & 30 \\
Schreiben & 6 & 5.42 & 7 & 6 & $2.8^{*}$ & 1.84 & $10^{*}$ & 8 \\
Gesamtnote Klausur 2 & 57.61 & 60.9 & 60.38 & 6.25 & $18.77^{*}$ & 15.46 & 74.5 & $75.5^{*}$ \\
Grammatik und Wortschatz & 37.8 & 40.83 & 39.13 & 42.5 & $15.6^{*}$ & 12.7 & $59.5^{*}$ & 53.25 \\
Leseverstehen & 19.8 & 20.07 & 21.5 & 21.25 & 4.48 & $4.72^{*}$ & 16 & $23.5^{*}$ \\
Englischeinstufungstest & 33.17 & 30.85 & 33.25 & 32 & 5.74 & $7.52^{*}$ & $28^{*}$ & 27.5 \\
\hline
\end{tabular}

Die Erhöhung der Heterogenität der EG deutet darauf hin, dass sich die Leistungsdifferenzierung innerhalb der EG im WS 2016-17 vergrößert hat. Um das zu veranschaulichen, wird die Gesamtnote der Klausur 1 von beiden Gruppen als Beispiel für ein Streudiagramm genommen. Das Diagramm (siehe Abbildung 11) stellt die Verteilung der Gesamtnote Klausur 1 beider Gruppen dar und zeigt demzufolge, dass die Ergebnisse vieler Studierende der KG zwischen 70 und 60 liegen, während die Noten der EG gestreuter sind. Die Note der EG sind entweder gut (höher als 70) oder schlecht (niedriger als 60). 

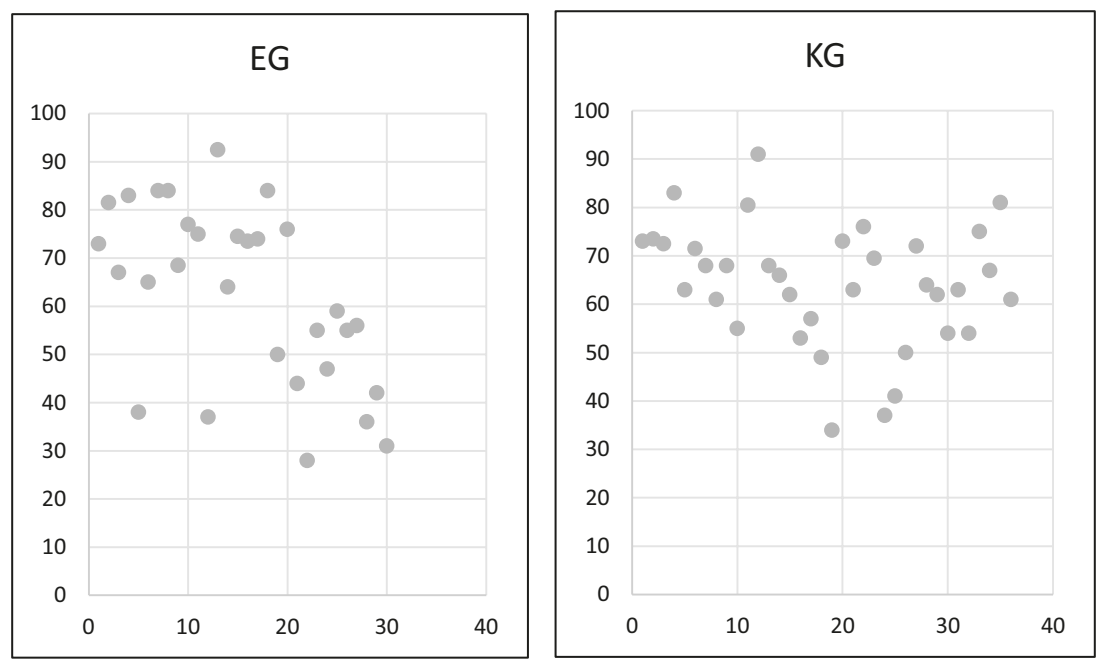

Abbildung 11: Streudiagramm von Gesamtnote der Klausur 1 WS 2016-17

Das könnte darauf schließen lassen, dass die neue Methode bzw. der Einsatz der Sprachlernspiele entweder sehr gut oder gar nicht akzeptiert wird. Man kann sich fragen, ob die Studierenden der EG mit der spielerischen Methode nicht zurecht kommen können. Deswegen ist es nötig, die Ergebnisse der Befragung zum Einsatz der Sprachlernspiele zuerst zu evaluieren, um die Unterschiedlichkeit vom Einfluss der neuen Methode näher zu betrachten.

\subsubsection{Auswertung und Analyse zu der Befragung zum Einsatz der Sprachlernspiele im Wintersemester 2016-17}

Die Befragung zum Einsatz der Sprachlernspiele (siehe Anhang 3) wird in der EG in der 14. Woche durchgeführt. Die Anonymität der Befragung wird bekannt gemacht. Die Studierenden wissen, dass die Befragung auf ihre Benotung keinen Einfluss ausüben kann. Die auf die Muttersprache der Lernenden hin verfasste Befragung wird auch durch einen Pretest von 4 Personen mehrmals überprüft und verbessert.

Insgesamt sind 21 Fragebogen in die Auswertung einbezogen.

Die Ergebnisse der ersten zwei Fragen zeigen, dass die Studierenden merken, dass eine neue Methode bzw. das Sprachlernspiel im Unterricht eingesetzt wird und die Interaktivität im Unterricht sehr hoch ist. 
Tabelle 22: Übersicht der Ergebnisse von Item 1 und 2 von der Befragung zum Einsatz der Sprachlernspiele WS 2016-17

\begin{tabular}{llllllc}
\hline Item & $\mathbf{1}$ & $\mathbf{2}$ & $\mathbf{3}$ & $\mathbf{4}$ & $\mathbf{5}$ & Mittelwert \\
\hline $\begin{array}{l}\text { 1.Haben Sie bemerkt, dass es Sprachlernspiele im } \\
\text { Unterricht gibt? }\end{array}$ & 1 & 1 & 1 & 2 & 16 & 4.48 \\
$\quad(1$ gar nicht -5 sehr deutlich) & & & & & & \\
$\begin{array}{l}\text { 2.Wie sieht die Interaktivität dieser Lehrmethode } \\
\begin{array}{l}\text { Ihrer Meinung nach aus? } \\
(1 \text { sehr schwach - } 5 \text { sehr hoch) }\end{array}\end{array}$ & 0 & 0 & 1 & 5 & 15 & 4.67 \\
\hline
\end{tabular}

Die Auswertung der Items über die Funktionen von Sprachlernspielen (siehe Tabelle 23) deutet darauf hin, dass die Lernenden zustimmen, dass Sprachlernspiele das Erlernen von Grammatik und Wortschatz interessanter machen, und sie angstabbauend sind. Sie sind der Meinung, dass Sprachlernspiele die sprachliche Kompetenz fördern, zum autonomen Lernen beitragen, und die Kommunikation in der Gruppe verbessern können.

Tabelle 23: Übersicht der Ergebnisse von Item 3 von der Befragung zum Einsatz der Sprachlernspiele WS 2016-17

$\begin{aligned} & \text { Stimmen Sie die folgenden Aussagen zum } \\ & \text { Sprachlernspiel zu? (1 gar nicht - } 4 \text { völlig) }\end{aligned}$
Sprachlernspiele...

\begin{tabular}{llllll}
\hline $3.1 \ldots$ machen Grammatiklernen interessanter. & 0 & 1 & 14 & 6 & 3.24 \\
3.2...machen Wortschatzlernen interessanter. & 0 & 4 & 10 & 7 & 3.14 \\
3.3....bauen Angst beim Sprechen ab. & 0 & 3 & 13 & 5 & 3.1 \\
3.4...erhöhen meine Sprechzeit auf Deutsch im & 0 & 2 & 14 & 5 & 3.14
\end{tabular}

Unterricht.

$\begin{array}{lllllll}\text { 3.5...erhöhen meine sprachliche Kompetenz im } & 0 & 3 & 14 & 4 & 3.05\end{array}$ allgemein

$\begin{array}{lllllll}\text { 3.6...verbessern die Kommunikation zwischen } & 0 & 0 & 15 & 6 & 3.29\end{array}$ mir und den Kommilitonen

$\begin{array}{lllllll}\text { 3.7...geben mir Chancen, im Unterricht Zeit für } & 0 & 2 & 15 & 4 & 3.1\end{array}$

Regelfinden zu haben und autonom zu denken.

$\begin{array}{lllllll}3.8 \ldots \text {...erhöhen die Autonomie beim Sprachlernen. } & 0 & & 7 & 11 & 3 & 2.81\end{array}$

3.9... erhöhen die Konzentration im Unterricht. $\quad \begin{array}{llllll}0 & 0 & 15 & 6 & 3.29\end{array}$

$\begin{array}{llllll}3.10 \ldots \text { bringen Abwechslung im Unterricht. } & 0 & 0 & 8 & 13 & 3.62\end{array}$

$\begin{array}{lllllll}3.11 \ldots \text { sind hilfreich für Fremdsprachenlernen im } & 0 & 0 & 13 & 8 & 3.38\end{array}$ allgemeinen.

$\begin{array}{llllll}3.12 \ldots \text { sind interessant, aber für mich etwas zu } & 3 & 11 & 5 & 2 & 2.29\end{array}$ schwer.

$\begin{array}{llllll}\text { 3.13... sind Zeitverschwendung im Unterricht. } & 11 & 10 & 0 & 0 & 1.48\end{array}$ 
Nach der Befragung kann man feststellen, dass Sprachlernspiele die Konzentration erhöhen und den Unterricht abwechslungsreicher machen können. Die Lernenden sind sich darin einig, dass Sprachlernspiele keine Zeitverschwendung im Unterricht sind und keine Überforderung verursachen.

Zur Einsatzzeit der Sprachlernspiele im Unterricht teilen die Studierenden verschiedene Meinungen. Die Mehrheit der Lernenden (10) ist der Meinung, dass die Spiele $50 \%$ bis $90 \%$ der Unterrichtszeit einnehmen sollen, weil Sprachlernspiele das Lernen und ihre Konzentration fördern können. 8 Lernende sind der Meinung, dass $30 \%$ bis $50 \%$ der Unterrichtszeit für Sprachlernspiele ausreichend ist, weil genügend Zeit für eine Zusammenfassung und die Faktenerklärung bleiben soll.

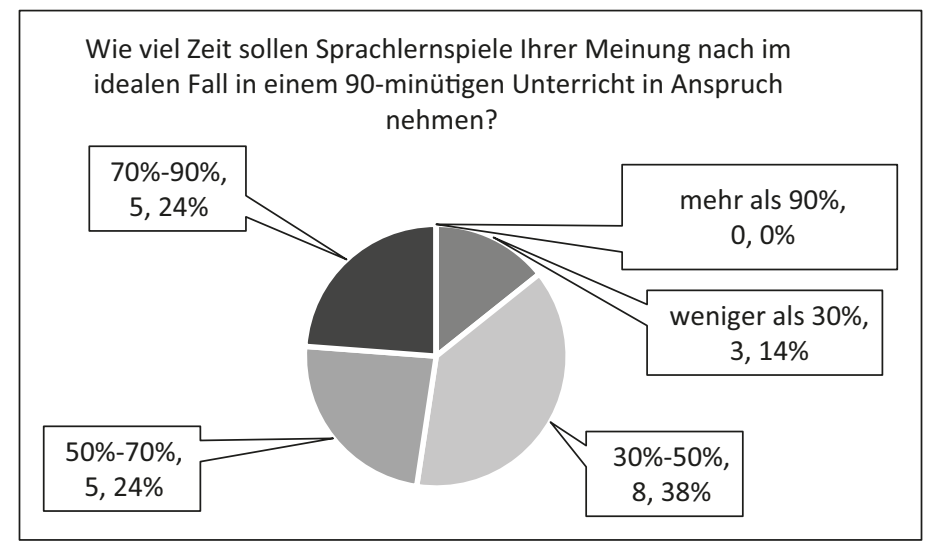

Abbildung 12: Die Ergebnisse von Item 6 der Befragung zum Einsatz der Sprachlernspiele WS 2016-17

Alle befragten Lernenden bevorzugen Unterricht mit Sprachlernspielen, und alle möchten, dass Sprachlernspiele im kommenden Semester eingesetzt werden.

Tabelle 24: Übersicht der Ergebnisse von Item 7 von der Befragung zum Einsatz der Sprachlernspiele WS 2016-17

\begin{tabular}{lll}
\hline $\begin{array}{l}\text { 7. Welche Unterrichtsform gefällt } \\
\text { Ihnen besser? }\end{array}$ & $\begin{array}{l}\text { Unterricht ohne } \\
\text { Sprachlernspiel }\end{array}$ & $\begin{array}{l}\text { Unterricht mit } \\
\text { Sprachlernspiel }\end{array}$ \\
\hline & 0 & 21 \\
\hline
\end{tabular}


Tabelle 25: Übersicht der Ergebnisse von Item 8 von der Befragung zum Einsatz der Sprachlernspiele WS 2016-17

\begin{tabular}{ll}
\hline $\begin{array}{l}\text { 8.Hoffen Sie, dass Sprachlernspiele im nächsten Semester } \\
\text { weiter benutzt werden? }\end{array}$ & Nein \\
\hline & 21 \\
\hline
\end{tabular}

Als Begründungen schreiben Sie:

Tabelle 26: Übersicht der Begründungen von Item 10 von der Befragung zum Einsatz der Sprachlernspiele WS 2016-17

\section{Bitte begründen Sie ihre Auswahl:}

Diese Methode ist viel besser als die in der Oberschule.

Es hilft uns, die Leistung zu verbessern.

Das Wissen ist leichter zu merken, und die Atmosphäre ist aktiver.

Sprachlernspiele helfen beim Lernen. $\times 5$

Beim Lernen spielen und im Spiel lernen ist mir eine nicht schlechte Idee.

Mehr Zeit für Kommunikation im Unterricht.

Erhöht mein Interesse fürs Lernen.

Ich kann ohne Stress lernen. $\times 2$

Ich kann mich besser konzentrieren.

Sprachlernspiele machen den Deutschunterricht besonders.

Zusammenfassend lässt sich sagen, dass die Effekte von Sprachlernspielen, die im Kapitel 3.3 diskutiert werden, weitgehend von den Lernenden wahrgenommen und akzeptiert werden. Studierende sind der Meinung, dass Sprachlernspiele dabei helfen können, das Gelernte in einer interessanten Weise besser und effizienter zu verstehen. Es fördert die Kommunikation und baut Angst ab. Alle wollen in der Zukunft weiter mit Sprachlernspielen lernen.

\subsubsection{Schlussfolgerung der Forschungsphase Wintersemester 2016-17}

Die Befragungsergebnisse zum Einsatz der Sprachlernspiele sprechen dafür, dass Sprachlernspiele hinsichtlich der Motivation und sprachlicher Leistung effizient sein sollen. Gegenüber dieser optimistischen subjektiven Einschätzung deuten die statistischen Ergebnisse der Prüfungen jedoch an, dass kein signifikanter Unterschied der Prüfungsleistungen der Gruppen festgestellt werden kann. Die 
Analyse der Befragung zur Motivation und Unterrichtsevaluation lässt erkennen, dass die EG eine erhöhte Interaktivität und Selbstevaluation zeigen, aber keine bessere Einstellung gegenüber Deutsch oder eine positivere Unterrichtsbewertung ${ }^{22}$ einnehmen.

Die Hypothese 1 „Lernende, die DaF-Unterricht mit Sprachlernspielen besuchen, sind in einer vergleichbaren Situation signifikant mehr motiviert, als Lernende in einem lehrerzentrierten DaF-Unterricht ohne Sprachlernspiele." wird teilweise bestätigt, während die Hypothese 2 „Lernende, die den DaF-Unterricht mit Sprachlernspielen besuchen, entwickeln in einer vergleichbaren Situation bessere sprachliche Kompetenzen als Lernende in einem lehrerzentrierten DaF-Unterricht ohne Sprachlernspiele." widerlegt wird. Darüber hinaus gibt es Anzeichen dafür, dass der Leistungsunterschied innerhalb der EG größer geworden ist, da die Heterogenität der Semesterprüfungsnoten von der EG größer als die von der KG ist.

Zusammenfassend lässt sich sagen, dass die Sprachlernspiele das subjektive Lernerlebnis der Probanden deutlich verbessern können. Diese Verbesserung muss jedoch nicht zu einem besseren Lernfortschritt führen.

Ein möglicher Grund könnte darin liegen, dass es bei den Prüfungen zu viele Störvariablen gibt. Die Effizienz von Sprachlernspielen hinsichtlich Lernfortschritt wird durch zwei Tests am Ende des Semesters überprüft. Es gibt allerdings sehr viele Störfaktoren im Test: Stress, Abschreiben usw. Eine einmalige Prüfung am Semesterende kann keinen Überblick des gesamten Lernfortschritts während des Semesters hervorbringen. Mehrere Tests zu verschiedenen Zeitphasen können wahrscheinlich ein umfassenderes Bild dafür liefern.

Darüber hinaus könnte die Lernerfahrung dabei auch eine wichtige Rolle spielen. Da Deutsch nicht die erste gelernte Fremdsprache der Zielgruppe ist, haben alle Studierende mindestens 6 Jahre Englisch im traditionellen lehrerzentrierten Fremdsprachunterricht gelernt. Zur Roll der Lernerfahrung pointiert Rösler (1994, S. 30-33):

Die Erfahrungen, die die Lernenden beim Lernen einer Fremdsprache schon gemacht haben, die vorherrschenden Lernverfahren ihrer Umgebung und die Veränderung von Weltsicht, die mit dem Lernen einer fremden Sprache einhergehen kann, eine bedeutende Rolle bei der Beschreibung von und vor allem bei Eingriffen in institutionell gesteuertes FL spielen sollten[...] Wer DaF lernt, hat zumindest schon seine eigene Muttersprache(und meistens eine andere Fremdsprache) erworben und sich im Laufe seiner Entwicklung ein Bild von der Welt gemacht, hat Routinen, Schemata, Denkweisen entwickelt, die beim Herangehen an die neue fremde Sprache Deutsch eine Rolle spielen. Weder beginnt er

22 Beide Gruppe haben den Unterricht als sehr gut bewertet. 
erstmalig zu denken oder Kategorien zu bilden, noch ist es das erste Mal, dass er mit Kultur in Berührung kommt. Diesem Sachverhalt ist in Unterrichtsverfahren und Lehrwerken oft nicht immer ausreichend Rechnung getragen worden...

Roche (2013, S. 39) spricht eben dafür, dass die Anpassungsphase für einen erfolgreichen kommunikativeren Fremdsprachenunterricht mit Lernenden, die nur Erfahrung mit lehrerzentriertem Unterricht haben, von Bedeutung ist. Wenn man davon ausgeht, dass die Lernerfahrung der Zielgruppe ihre Leistung und Motivation stark beeinflussen kann, soll damit begründet werden, dass die Lernenden der EG eine neue Methode in 11 Wochen nicht völlig akzeptieren können. Der Leistungsstand der KG, die mit einer vertrauten Methode lernen, ist stabiler, während die EG Zeit braucht, sich die neue Methode anzupassen.

Die Tatsache, dass beide Gruppen den Unterricht gut bewertet haben, spricht dafür, dass beide Gruppen mit der Vermittlungsmethode zufrieden sind. Dieses Resultat soll näher betrachtet werden. Erstens kann man damit begründen, dass die Studierenden der EG mit der spielerischen Methode zufrieden sind. Zweitens ist die Zufriedenheit der KG eben auf Lernerfahrung zurückzuführen: Die konventionelle lehrerzentrierte Methode ist ihnen vertraut. Sie wissen nicht, dass ein Fremdsprachenunterricht anders bzw. interaktiv/kommunikativ und spielerisch gestaltet werden kann. Die niedrige Interaktivität und die lehrerzentrierte Methode führen deswegen zu keiner schlechten Unterrichtsbewertung.

Um herauszufinden, ob die Leistungs- und Motivationserhöhung durch die Lernerfahrung beeinflusst werden, ist es nötig, die Aktionsforschung fortzusetzen. Die Fortsetzung hilft dabei, die Wirkung der Sprachlernspiele in einer längeren Phase näher zu beobachten und zu analysieren.

\subsection{Forschungsphase Sommersemester 2017}

\subsubsection{Forschungsdesign im Sommersemester 2017}

Da der Einfluss der Lernerfahrung nicht außer Acht gelassen werden kann, soll eine zweite Phase der Aktionsforschung geplant werden. Da die zweite Phase als eine Verlängerung der ersten Phase betrachtet werden kann, soll die Gruppenverteilung identisch bleiben, nämlich Gruppe A als die KG und Gruppe B als die EG.

Durch die Befragung zum Einsatz der Sprachlernspiele im WS 2016-17 kann man festhalten, dass die eingesetzten Sprachlernspiele von den Lernenden positiv bewertet werden. Darüber hinaus weist die Auswertung der Befragung zur Motivation und Unterrichtsevaluation nach, dass die Lernenden beider Gruppen mit 
dem Unterricht zufrieden sind. Infolgedessen muss keine Änderung hinsichtlich der Didaktisierung und Spieladaptionsmethode vorgenommen werden.

Das heißt, dass die Probanden, die Untersuchungsmaterialien, die Adaption von Übungen als Spiele und die Unterrichtsplanung im SS 2017 die gleichen wie im WS 2016-17 sind. Es wird weiter mit den Lehrwerk-Reihen „Klick auf Deutsch" und deren zugehörigen Übungsheften gearbeitet.

Am Ende des SS 2017 soll die gleiche Befragung zur Motivation und Unterrichtsevaluation sowie zum Einsatz der Sprachlernspiele aus WS 2016-17 durchgeführt werden, um mögliche Änderungen bei der Motivation, der Unterrichtsbewertung und dem Einsatz der Sprachlernspiele zu überprüfen.

In der zweiten Phase der Aktionsforschung sollen allerdings verstärkt Datenerhebungsmethoden für die Lernleistung verwendet werden, weil eine einmalige Prüfung am Ende des Semesters zu viele Störvariablen enthalten kann. Um die Störfaktoren zu relativieren, wird geplant, am Anfang des Semesters und nach dem Erlernen jeder Einheit einen C-Test in beiden Gruppen durchzuführen. Ein C-Test basiert auf dem Konzept der reduzierten Redundanz und soll den allgemeinen Sprachstand erfassen, gilt als objektiv und ist einfach in der Herstellung und Auswertung (vgl. Porsch 2014, S. 90; Huneke und Steinig 2013, S. 251).

Außerdem werden Kolleginnen zu beliebigen Sitzungen eingeladen, um den Unterricht zu beobachten. Damit kann herausgefunden werden, ob bei den Unterrichtsverläufen in den beiden Gruppen beobachtbare Unterschiede von Lehrenden festgestellt werden können, sodass die Effizienz des Sprachlernspiels aus einer Lehrenden-Perspektive evaluiert werden kann.

Die Befragung zur Motivation und Unterrichtsevaluation für beide Gruppen ist die gleiche wie im WS 2016-17 und wird zunächst analysiert. Zum Lernfortschritt beider Gruppen wird hier nicht nur die Semesterprüfung berücksichtigt, sondern auch die Ergebnisse der C-Tests werden analysiert. Dann kommt die Befragung zum Einsatz der Sprachlernspiele (siehe Anhang 4), die teilweise je nach den eingesetzten Spielformen des SS 2017 geändert wird, weil die Übungsformen und Übungsziele des SS 2017 anders sind als die im WS 2016-17. Schließlich werden die Daten der Unterrichtsbeobachtung der Lehrenden unter die Lupe genommen, um die Meinungen der Lehrerseite zu sammeln. 


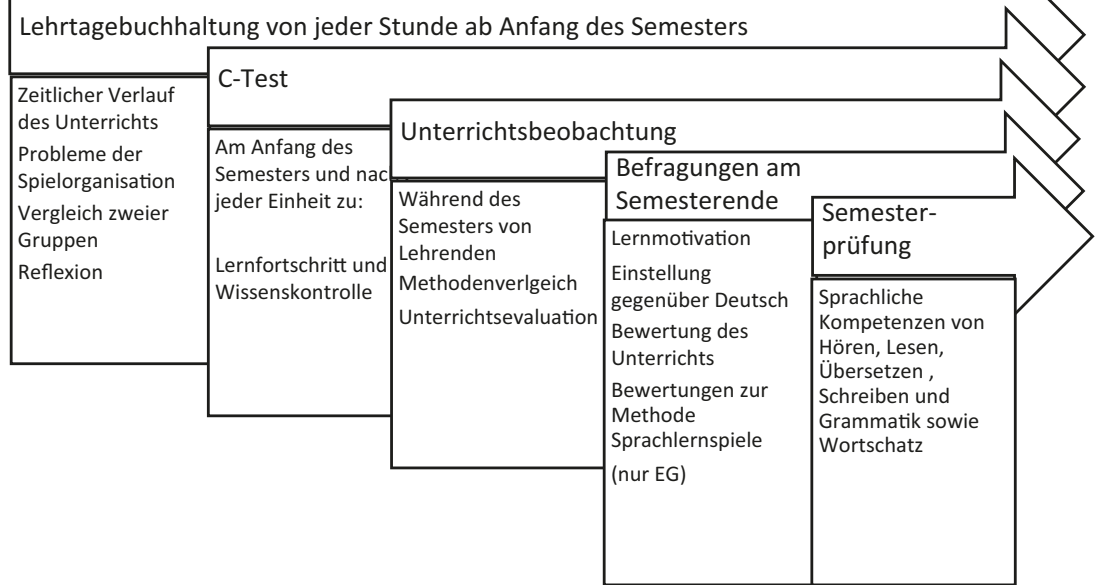

Abbildung 13: Übersicht der Datenerhebungsmethoden SS 2017

\subsubsection{Auswertung und Analyse zu der Befragung zur Motivation und Unterrichtsevaluation im Sommersemester 2017}

Die schriftliche Befragung zur Motivation und Unterrichtsevaluation wird am 2. Juni in der 14. Unterrichtswoche im Unterricht durchgeführt. Die Anonymität der Befragung wird bekannt gemacht. Die Fragen sind identisch mit der Befragung im WS 2016-2017.

Insgesamt werden 60 beantwortete Fragebogen in die Auswertung einbezogen. 25 von der EG, 35 von der KG.

Die gerichteten Hypothesen sind identisch mit den vom WS 2016-17:

1. Die EG ist mehr motiviert als die KG.

2. Die EG gibt dem Unterricht eine bessere Bewertung als die $K G$.

3. Der Unterricht der EG wird interaktiver als der von der KG bewertet.

Durch den MWU-Test mit SPSS kann man bei Item 5.3, 5.5, 5.6, 6, 7.2, 8.4 und 8.8 signifikante Unterschiede beobachten (siehe Anhang 8): 
Tabelle 27: Übersicht der Items mit signifikantem Unterschied von der Befragung zur Motivation und Unterrichtsevaluation SS 2017

\begin{tabular}{|c|c|}
\hline $\begin{array}{l}\text { Item } \\
\text { Nr. }\end{array}$ & Inhalt \\
\hline 5.3 & $\begin{array}{l}\text { Im Deutschunterricht habe ich genügend Zeit, mit meinen Kommilitonen zu } \\
\text { kommunizieren. (1 ich stimme gar nicht } \mathrm{zu}-5 \text { ich stimme völlig zu) }\end{array}$ \\
\hline 5.5 & $\begin{array}{l}\text { Im Deutschunterricht mache ich die Aufgaben meistens mit meinen Kommilitonen } \\
\text { zusammen. ( } 1 \text { ich stimme gar nicht } \mathrm{zu}-5 \text { ich stimme völlig zu) }\end{array}$ \\
\hline 5.6 & $\begin{array}{l}\text { Im Deutschunterricht mache ich die Aufgaben meistens allein. ( } 1 \text { ich stimme gar } \\
\text { nicht zu - } 5 \text { ich stimme völlig zu) }\end{array}$ \\
\hline 6 & $\begin{array}{l}\text { Bitte bewerten Sie die Interaktivität im Unterricht im Allgemeinen. (1 sehr } \\
\text { schwach-5 sehr stark) }\end{array}$ \\
\hline 7.2 & $\begin{array}{l}\text { Ich finde, dass der Deutschunterricht mir geholfen hat, die Welt kennenzulernen } \\
\text { und zu verstehen. ( } 1 \text { ich stimme gar nicht } \mathrm{zu}-5 \text { ich stimme völlig zu) }\end{array}$ \\
\hline 8.4 & $\begin{array}{l}\text { Bitte bewerten Sie, ob folgende Faktoren im Unterricht zeitlich richtig behandelt } \\
\text { werden: Übung zum Hörverständnis. (1 zu wenig Zeit- } 5 \text { zu viel Zeit) }\end{array}$ \\
\hline 8.8 & $\begin{array}{l}\text { Bitte bewerten Sie, ob folgende Faktoren im Unterricht zeitlich richtig behandelt } \\
\text { werden: Übungen zur Kommunikationskompetenz. ( } 1 \text { zu wenig Zeit- } 5 \text { zu viel Zeit) }\end{array}$ \\
\hline
\end{tabular}

Tabelle 28: Ergebnisse von MWU-Tests von Items aus Tabelle 27

\begin{tabular}{llllllll}
\hline Item & \multicolumn{2}{c}{$\begin{array}{c}\text { Mittelwert } \\
\text { (Rohdaten) }\end{array}$} & $\begin{array}{c}\text { Mann- } \\
\text { Whitney-U }\end{array}$ & $\begin{array}{c}\text { Wilcoxon } \\
\text { W }\end{array}$ & Z & $\begin{array}{c}\text { Asymp. Sig. } \\
\text { (zweiteilig) }\end{array}$ & $\begin{array}{c}\text { P. } \\
(=\text { Sig./ 2) }\end{array}$ \\
\cline { 2 - 5 } & EG(25) & KG(35) & & & & & \\
\hline 5.3 & 3.16 & 2.57 & 300.500 & 930.500 & -2.165 & .030 & $0.015<0.05^{\star}$ \\
5.5 & 3.56 & 2.66 & 247.500 & 877.500 & -2.951 & .003 & $0.0015<0.05^{\star}$ \\
5.6 & 2.84 & 3.4 & 283.500 & 608.500 & -2.433 & .015 & $0.0075<0.01^{*}$ \\
6 & 4.4 & 3.74 & 230.000 & 860.000 & -3.322 & .001 & $0.0005<0.01^{\star}$ \\
7.2 & 4.23 & 3.8 & 296.000 & 621.000 & -2.355 & .019 & $0.0095<0.01^{\star}$ \\
8.4 & 2.24 & 2.51 & 335.500 & 660.500 & -1.699 & .089 & $0.0445<0.05^{\star}$ \\
8.8 & 3.16 & 2.74 & 335.000 & 965.000 & -1.837 & .066 & $0.033<0.05^{\star}$ \\
\hline
\end{tabular}

Die einteilige Signifikanz P von Item 5.3, 5.5 und 5.6 zeigt, dass die EG mehr Gelegenheit zur Kommunikation mit Kommilitonen als die KG hat. Die EG hat mehr Zeit, mit Kommilitonen zu kommunizieren und arbeitet oft mit anderen zusammen, während die KG wenig Zeit zur Kommunikation hat und die Aufgaben eher allein macht.

Das Ergebnis von Item 6 bestätigt eben, dass die Interaktivität im Unterricht von der EG signifikant höher ist als die in der KG. 
Das Ergebnis von Item 7.2 deutet auch darauf hin, dass Lernende aus der EG im Deutschunterricht die Welt besser kennengelernt haben als die aus der KG. Das heißt, dass die EG mehr Gelegenheit als die KG hat, ihre interkulturelle Kompetenz zu verbessern.

Item 8.8 bestätigt, dass im Unterricht der EG mehr Übungszeit der Kommunikationskompetenz als im Unterricht der KG gewidmet wird.

Aus den Rohdaten von Item 8.4 kann man erschließen, dass beide Gruppen mit der Übungszeit zum Hörverstehen zufrieden sind. Laut der einteiligen Signifikanz P von Item $8.4(\mathrm{p}=0.089 / 2=0.0445<0.05)$ kann man allerdings feststellen, dass die Lernenden der EG mehr Übungszeit für Hörverstehen haben möchten.

Die Implikation dieser Ergebnisse ist vielfältig. Erstens ist es deutlich, dass der Vermittlungsweg mit Sprachlernspielen hoch interaktiv ist. Hypothese 3 „Der Unterricht der EG wird interaktiver als der von der KG bewertet. “ wird bestätigt. Zweitens kann der Beitrag von Sprachlernspielen zur interkulturellen Kommunikation und Kommunikationskompetenz teilweise bestätigt werden. Zusätzlich kann man feststellen, dass die EG Hörverstehen-Übung wichtiger als die KG wahrnehmen, weil sie die Übungszeit für ungenügend hält.

Es wird kein statistisch signifikanter Unterschied zwischen den Gruppen bei den Items zur Einstellung gegenüber der deutschen Sprache sowie zur Bewertung der eigenen Sprachlernfähigkeit oder Lernzeit außerhalb des Unterrichts festgestellt.

Eine Änderung kann man allerdings bei Item 1.3 beobachten. Bei Item 1.3 „Wenn ich kann, will ich lieber Englisch lernen. (1 ich stimme gar nicht zu - 5 ich stimme völlig zu) " hat man im WS 2016-17 einen signifikanten Unterschied zwischen den zwei Gruppen festgestellt (siehe Kapitel 5.1.2): Die EG gab im Vergleich zur KG Englisch den Vorzug vor Deutsch. Im SS 2017 zeigen beide Gruppen aber keinen Unterschied mehr. Wenn man die Mittelwerte von Item 1.3 der beiden Semester näher betrachtet, kann man feststellen: Der Vorzug von der EG, die englische Sprache zu lernen, hat sich verringert, während der von der KG sich erhöht hat ${ }^{23}$. Das spricht dafür, dass sich die Einstellung der EG gegenüber der deutschen Sprache ins Positive gewandelt, während sich die der KG in die andere (negative) Richtung entwickelt hat.

23 An dieser Stelle soll nochmal betont werden, dass mehr als die Hälfte von EG Englisch lernen wollten, aber sich nicht ummelden konnten. 
Tabelle 29: Vergleich der Mittelwerte von Item 1.3 WS 2016-17 und SS 2017

\begin{tabular}{lccc}
\hline Gruppe & $\begin{array}{c}\text { Mittelwert } \\
\text { WS 2016-17 }\end{array}$ & $\begin{array}{c}\text { Mittelwert } \\
\text { SS 2017 }\end{array}$ & Vorzug von Englisch \\
\hline EG & 3.0769 & 2.96 & $\downarrow$ \\
KG & 2.3000 & 2.9143 & $\uparrow$ \\
\hline
\end{tabular}

Die Hypothese 1 „Die EG ist mehr motiviert als die KG. “ soll anhand dieser Ergebnisse teilweise bestätigt werden.

Die Hypothese 2 „Die EG gibt dem Unterricht eine bessere Bewertung als die $K G$ “ wird widerlegt. Die Ergebnisse von zwei Semestern deuten deutlich darauf hin, dass die Unterrichtsbewertung von Studierenden mit dem Vermittlungsweg keinen Zusammenhang hat. Beide Gruppen haben den Unterricht gut bewertet, und es kann kein statistischer Unterschied gefunden werden:

Tabelle 30: Ergebnisse vom MWU-Test von Item 9 im SS 2017

\begin{tabular}{|c|c|c|c|c|c|c|}
\hline \multirow[t]{2}{*}{$\begin{array}{l}\text { Item } 9 \\
\text { Bitte bewerten Sie } \\
\text { Deutschunterricht im } \\
\text { Allgemeinen. (1 sehr } \\
\text { schlecht-5 sehr gut) }\end{array}$} & \begin{tabular}{ll}
\multicolumn{2}{c}{ Mittelwert } \\
EG & KG \\
$(25)$ & $(35)$
\end{tabular} & \multirow[t]{2}{*}{$\begin{array}{c}\text { Mann- } \\
\text { Whitney- } \\
-\quad \mathrm{U}\end{array}$} & $\begin{array}{c}\text { Wilcoxon } \\
\text { W }\end{array}$ & $\mathrm{Z}$ & $\begin{array}{l}\text { Asymp. Sig. } \\
\text { (zweiteilig) }\end{array}$ & $\begin{array}{c}\text { P. } \\
\text { (= Exact } \\
\text { Sig./ 2) }\end{array}$ \\
\hline & $\begin{array}{ll}3.77 & 4.12\end{array}$ & & 973.000 & -1.555 & .12 & $0.06>0.05$ \\
\hline
\end{tabular}

Im Anschluss daran soll herausgefunden werden, ob es einen statistisch signifikanten Unterschied bei Leistungen der beiden Gruppen im SS 2017 gibt.

\subsubsection{Auswertung und Analyse zu den Ergebnissen der Semesterprüfung im Sommersemester 2017}

Die Prüfung im SS 2017 findet nach 16- wöchiger Unterrichtszeit in der 17. Woche statt. Die Prüfung ist genau so wie die vom WS 2016-17, aus zwei Klausuren gestaltet.

Tabelle 31: Übersicht der Notenverteilung der Semesterprüfung SS 2017

\begin{tabular}{lccc}
\hline Klausur 1 & $\begin{array}{c}\text { Gesamtpunkt } \\
\text { Teilfertigkeiten }\end{array}$ & Klausur 2 & $\begin{array}{c}\text { Gesamtpunkt } \\
\text { Teilfertigkeiten }\end{array}$ \\
\hline Hörverstehen & 35 & $\begin{array}{c}\text { Grammatik und } \\
\text { Wortschatz }\end{array}$ & 80 \\
Übersetzen & 50 & $\begin{array}{c}\text { Leseverstehen } \\
\text { Schreiben }\end{array}$ & \\
\hline
\end{tabular}


Die Semesterprüfung SS 2017 findet am 19. Jun. 2017 in zwei Seminarräumen gleichzeitig statt. In jedem Raum gibt es 2 Aufsichtslehrende zur Organisation und Beaufsichtigung der Prüfung. Der Verlauf und die zur Verfügung stehende Zeit der Prüfung sind identisch wie im WS 2016-17.

Wie im WS 2016-17, entsprechen der Inhalt der Prüfung und die Struktur der Aufgaben den gelernten Einheiten und durchgeführten Übungen vom SS 2017. Insgesamt haben 34 Studierende aus der KG und 25 Studierende aus der EG die Prüfung absolviert.

Folgende Hypothese wird gestellt und soll überprüft werden:

Die EG mit Sprachlernspielen zeigt bessere Leistung als die KG ohne Sprachlernspiele.

Die Testergebnisse werden mit SPSS durch MWU-Test überprüft und die Ergebnisse sind wie folgt ${ }^{24}$ :

Tabelle 32: Ergebnisse von MWU-Tests der Semesterprüfung SS 2017

\begin{tabular}{lccccccc}
\hline Prüfung & \multicolumn{2}{c}{ Mittelwert } & $\begin{array}{c}\text { Mann- } \\
\text { Whitney-U }\end{array}$ & $\begin{array}{c}\text { Wilcoxon } \\
\text { W }\end{array}$ & $\begin{array}{c}\text { Z } \\
\text { Asymp. } \\
\text { Sig.. } \\
\text { (zweiteilig) }\end{array}$ & $\begin{array}{c}\text { P. } \\
\text { (= Sig./ 2) }\end{array}$ \\
\hline Klausur 1 & $48.7200^{*}$ & 47.8235 & 400.000 & 995.000 & -.384 & .701 & $0.3505>0.05$ \\
Klausur 2 & $49.6800^{*}$ & 46.6471 & 378.000 & 973.000 & -.721 & .471 & $0.2355>0.05$ \\
\hline
\end{tabular}

Tabelle 33: Ergebnisse von MWU-Tests der Teilfertigkeiten SS 2017

\begin{tabular}{|c|c|c|c|c|c|c|c|}
\hline \multirow{2}{*}{$\begin{array}{l}\text { Teilkompe- } \\
\text { tenz }\end{array}$} & \multicolumn{2}{|c|}{ Mittelwert } & \multirow{2}{*}{$\begin{array}{c}\text { Mann- } \\
\text { Whitney-U }\end{array}$} & \multirow{2}{*}{$\begin{array}{c}\text { Wilcoxon } \\
\text { W }\end{array}$} & \multirow[t]{2}{*}{$\mathbf{Z}$} & \multirow{2}{*}{$\begin{array}{l}\text { Asymp. } \\
\text { Sig. (zwei- } \\
\text { teilig) }\end{array}$} & \multirow{2}{*}{$\begin{array}{c}\text { P. } \\
\text { (= Sig./ 2) }\end{array}$} \\
\hline & EG(25) & KG(34) & & & & & \\
\hline $\begin{array}{l}\text { Hörverste- } \\
\text { hen }\end{array}$ & $18.0400^{*}$ & 15.6176 & 301.000 & 896.000 & -1.909 & .056 & $\begin{array}{l}0.028 \\
<0.05^{*}\end{array}$ \\
\hline Übersetzen & 20.1600 & $20.5294^{*}$ & 411.000 & 736.000 & -.215 & .830 & $0.415>0.05$ \\
\hline Schreiben & 10.5200 & $11.6765^{\star}$ & 374.500 & 699.500 & -.793 & .428 & $0.214>0.05$ \\
\hline $\begin{array}{l}\text { Grammatik } \\
\text { und Wort- } \\
\text { schatz }\end{array}$ & $34.2400^{*}$ & 30.7647 & 344.500 & 939.500 & -1.236 & .217 & $\begin{array}{r}0.1085 \\
>0.05\end{array}$ \\
\hline $\begin{array}{l}\text { Leseverste- } \\
\text { hen }\end{array}$ & 15.4400 & $15.8824^{\star}$ & 383.500 & 708.500 & -.646 & .518 & $0.259>0.05$ \\
\hline
\end{tabular}

24 Rohdaten der Prüfung siehe Anhang 14. 
Ein Blick auf die Ergebnisse zeigt, dass kein statistisch signifikanter Unterschied bei der Gesamtnote zweier Klausuren bestätigt werden kann, obwohl die Mittelwerte der Klausurleistungen von der EG leicht höher als die von der KG sind.

Wenn man die Leistungen der Teilkompetenzen beider Gruppen betrachtet (siehe Tabelle 33), kann man einen einzigen signifikanten Unterschied bei der Fertigkeit Hörverstehen finden: $\mathrm{P}=0.056 / 2=0.028<0.05$. Das heißt, dass die EG beim Hörverstehen besser als die KG abschneidet.

Der Index der lexikalischen Vielfalt der Leistungen (siehe Tabelle 34) zeigt: Die Klausurnoten der EG weisen eine höhere Streuung auf als die der KG, was an den höheren Werten für die Standardabweichung und die Spannweite zu erkennen ist. Das heißt, dass die Heterogenität der Klausurnoten von der EG bezüglich der lexikalischen Vielfalt größer als die von der KG ist, wie schon im WS 2016-17.

Tabelle 34: Übersicht des Index lexikalischer Vielfalt der Semesterprüfung SS 2017

\begin{tabular}{lcccccccc}
\hline Index & \multicolumn{1}{c}{ Mittelwert } & Median & \multicolumn{3}{c}{$\begin{array}{c}\text { Standardabwei- } \\
\text { chung }\end{array}$} & \multicolumn{3}{c}{ Spannweite } \\
\hline Gruppe & EG & KG & EG & KG & EG & KG & EG & KG \\
& $(25)$ & $(35)$ & $(25)$ & $(35)$ & $(25)$ & $(35)$ & $(25)$ & $(35)$ \\
Gesamtnote Klausur 1 & 48.72 & 47.82 & 52 & 44.5 & $16.04^{*}$ & 16.00 & 60 & 60 \\
Hörverstehen & 18.04 & 15.62 & 18 & 16 & 3.90 & $5.72^{*}$ & 15 & $26^{*}$ \\
Übersetzen & 20.16 & 20.53 & 21 & 19 & $12.75^{*}$ & 10.97 & $43^{*}$ & 37 \\
schreiben & 10.52 & 11.68 & 12 & 13 & $4.31^{*}$ & 2.37 & $14^{*}$ & 9 \\
Gesamtnote Klausur 2 & 49.69 & 46.65 & 48 & 44 & $15.64^{*}$ & 11.94 & $72^{*}$ & 55 \\
Grammatik und Wortschatz & 34.24 & 30.76 & 34 & 28 & $14.62^{*}$ & 11.85 & $68^{*}$ & 55 \\
Leseverstehen & 15.44 & 15.88 & 16 & 16 & $2.89^{*}$ & 2.28 & $14^{*}$ & 12 \\
\hline
\end{tabular}

Zusammenfassend lässt sich sagen, dass die Semesterprüfungsergebnisse vom SS 2017 keinen signifikanten Unterschied zwischen beiden Gruppen zeigen. Die Leistungsdifferenzierung innerhalb der EG ist im SS 2017 immer noch höher als die der KG. Die Hypothese „Die EG mit Sprachlernspielen zeigt bessere Leistung als die KG ohne Sprachlernspiele“ wird nur bei Leistung vom Hörverstehen bestätigt.

Im SS 2017 werden außer der Semesterprüfung noch C-Tests durchgeführt, um den Leistungsunterschied besser zu vergleichen. Im kommenden Unterkapitel werden die Ergebnisse von C-Tests ausgewertet und analysiert. 


\subsubsection{Auswertung und Analyse zu den Ergebnissen der C-Tests im Sommersemester 2017}

Durch die Untersuchung im WS 2016-17 sind die verborgenen Störfaktoren der Semesterprüfung hervorgehoben: Abschreiben, mangelnde Vorbereitung, Gesundheitszustand der Prüfungsteilnehmenden etc.

Es reicht nicht aus, die Effizienz von Sprachlernspielen im ganzen Semester mit einer einmaligen Prüfung zu überprüfen. Da die aktuelle Performanz eines Lerners nach Zeitpunkt der Erhebung, Aufgabenstellung, Testformat und Zusammensetzung der Lernergruppe stark variieren kann, sollen die Lernfortschritte über einen längeren Zeitraum mehrfach evaluiert werden (vgl. Vollmer 2007, S. 367). Daher wurden im SS 2017 fünf C-Tests während des Semesters durchgeführt, um die Lernfortschritte in einer repräsentativen Weise zu erfassen.

C-Tests werden oft wegen seiner Objektivität als Forschungsinstrument benutzt und gelten als leicht zu erstellen und einfach auszuwerten (vgl. Huneke und Steinig 2013, S. 251). Porsch (2014, S. 90) und Vollmer (2007, S. 367) erwähnen, dass der C-Test eine Form eines weiterentwickelten Cloze-Tests ist und den allgemeinen Sprachstand erfassen soll.

Im SS 2017 werden am Anfang des Semesters und nach jeder gelernten Einheit insgesamt $5 \mathrm{C}$-Tests im Unterricht durchgeführt, die jeweils aus 5 kurzen Texten bestehen. Der Textinhalt des ersten C-Tests basiert auf dem Wissen des WS 2016-17. Die anderen vier Tests entsprechen dem Inhalt der erlernten Einheit. Jeder kurze Text hat 20 Lücken und ist nach dem Konstruktionsprinzip des C-Tests (vgl. Grotjahn 1995, 38) gestaltet (Beispiel siehe Anhang 9). Die Tests finden am gleichen Tag in zwei Gruppen statt und dauern jeweils nicht mehr als 30 Minuten. Die Studierenden werden darauf hingewiesen, dass die Noten der C-Tests keinen Einfluss auf die Bewertung ihrer Semesterleistung ausüben, sodass Abschreiben möglicherweise vermieden werden kann.

Hier werden die Ergebnisse der C-Tests ${ }^{25}$ mit SPSS analysiert, um herauszufinden, ob die zwei Gruppen in den Tests anders abschneiden. Da nicht alle Studierenden an jedem Testtag anwesend sind, können hier diverse Teilnehmerzahlen auftauchen.

25 Rohdaten siehe Anhang 13. 
Tabelle 35: Ergebnisse von MWU-Tests von C-Tests SS 2017

\begin{tabular}{llllllll}
\hline Prüfung & \multicolumn{2}{c}{ Mittelwert } & $\begin{array}{c}\text { Mann- } \\
\text { Whitney-U }\end{array}$ & $\begin{array}{c}\text { Wilcoxon } \\
\text { W }\end{array}$ & $\begin{array}{c}\text { Z } \\
\text { Asymp. Sig. } \\
\text { (zweiteilig) }\end{array}$ & $\begin{array}{c}\text { P. } \\
\text { (= Sig./ 2) }\end{array}$ \\
\hline C-Test 1 & EG (26) & KG (35) & 385.500 & 1015.500 & -1.014 & .310 & 0.155 \\
$(\mathbf{2 7 . 0 2 . 2 0 1 7 )})$ & $20.0769^{*}$ & 16.4857 & & & & & $>0.05$ \\
C-Test 2 & EG (25) & KG (34) & 405.000 & 1000.000 & -.307 & .759 & 0.3795 \\
$(\mathbf{3 1 . 0 3 . 2 0 1 7 )})$ & $23.0800^{*}$ & 22.4706 & & & & & $>0.05$ \\
C-Test 3 & EG (25) & KG (28) & 267.000 & 673.000 & -1.481 .139 & 0.0695 \\
$(\mathbf{1 7 . 0 4 . 2 0 1 7 )}$ & $24.8400^{*}$ & 19.6429 & & & & $>0.05$ \\
C-Test 4 & EG (20) & KG (32) & 239.000 & 767.000 & -1.525 & .127 & 0.0635 \\
$(\mathbf{0 8 . 0 5 . 2 0 1 7 )}$ & $31.7500^{*}$ & 26.2813 & & & & $>0.05$ \\
C-Test 5 & EG (22) & KG (27) & 177.000 & 555.000 & -2.414 & .016 & 0.008 \\
$(\mathbf{2 9 . 0 5 . 2 0 1 7 )}$ & $32.3636^{*}$ & 23.9630 & & & & $<0.01^{*}$ \\
\hline
\end{tabular}

Die oberen statistischen Ergebnisse zeigen, dass sich der Unterschied der zwei Gruppen während des Semesters vergrößert. Die einteilige Signifikanz P vom C-Test $1 \mathrm{im}$ Februar beträgt 0.155 und deutet auf keinen Unterschied hin, während die einteilige Signifikanz P vom C-Test 5 im Mai einen statistisch sehr signifikanten Unterschied zeigt $(\mathrm{P}=0.016 / 2=0.008<0.01)$.

Wenn man die Mittelwerte jedes Tests beobachtet, kann man feststellen: Die Studierenden der EG haben in jedem Test bessere Noten bekommen. Bis Ende des Semesters ist die Note von der EG statistisch signifikant deutlich besser als die der KG.

Die Hypothese „Die EG mit Sprachlernspielen zeigt bessere Leistung als die KG ohne Sprachlernspiele" wird am Ende des Semesters bei einem C-Test bestätigt.

\subsubsection{Auswertung und Analyse zu der Befragung zum Einsatz der Sprachlernspiele im Sommersemester 2017}

Die Befragung zum Einsatz der Sprachlernspiele wird in der EG in der 15. Woche durchgeführt. Die Anonymität der Befragung wird bekannt gemacht. Die Lernenden wissen, dass die Befragung keinen Einfluss auf ihre Benotung ausüben kann.

Die Mehrheit der Items besteht aus der gleichen Befragung wie im WS 2016-17. Eine Frage zu ihrer Erfahrung zu Sprachlernspielen (Item 11), eine Frage zur zeitlichen Angemessenheit von Sprachlernspielen im Unterricht (Item 7) und eine Frage zum zukünftigen Methodenwunsch außer Sprachlernspielen (Item 13) werden hinzugefügt. Außerdem sind die Spiele anders als die im WS 
2016-17, weil der Lerninhalt und dazugehörige Übungen anders sind. Die Fragen zu den Einzelheiten der Spiele bleiben identisch mit den aus dem vorherigen Semester (siehe Anhang 4).

Im SS 2017 sind insgesamt 25 Fragebögen in die Auswertung einbezogen. Die Ergebnisse werden mit SPSS durch MWU-Test analysiert, um mögliche Unterschiede zwischen WS 2016-17 und SS 2017 zu finden. Zu den vollständigen Analyseergebnissen siehe Anhang 10.

Die Ergebnisse der ersten zwei Fragen zeigen keinen statistisch signifikanten Unterschied zu den Ergebnissen des WS 2016-17. Das heißt, dass die Studierenden die Präsenz der eingesetzten Methode im Unterricht merken und zustimmen, dass die Interaktivität im Unterricht sehr hoch ist:

Tabelle 36: Ergebnisse von MWU-Tests zwei Semestern von Item 1 und 2

\begin{tabular}{|c|c|c|c|c|c|c|c|}
\hline \multirow[t]{2}{*}{ Item } & \multicolumn{2}{|c|}{ Mittelwert } & \multirow{2}{*}{$\begin{array}{c}\text { Mann- } \\
\text { Whitney-U }\end{array}$} & \multirow{2}{*}{$\begin{array}{c}\text { Wilcoxon } \\
\text { W }\end{array}$} & \multirow[t]{2}{*}{$\mathrm{Z}$} & \multirow{2}{*}{$\begin{array}{l}\text { Asymp. Sig. } \\
\text { (zweiteilig) }\end{array}$} & \multirow{2}{*}{$\begin{array}{c}\text { P. } \\
(=\text { Sig./ } \\
\text { 2) }\end{array}$} \\
\hline & WS & $\overline{\text { SS }}$ & & & & & \\
\hline $\begin{array}{l}\text { 1. Haben Sie bemerkt, } \\
\text { dass es Sprachlernspiele } \\
\text { im Unterricht gibt? } \\
\text { ( } 1 \text { gar nicht- } 5 \text { sehr } \\
\text { deutlich)? }\end{array}$ & 4.48 & 4.68 & 260.500 & 585.500 & -.057 & .954 & $\begin{array}{c}0.477 \\
>0.05\end{array}$ \\
\hline $\begin{array}{l}\text { 2. Wie sieht die Inter- } \\
\text { aktivität dieser Lehr- } \\
\text { methode Ihrer Meinung } \\
\text { nach aus? } \\
\text { ( } 1 \text { sehr schwach-5sehr } \\
\text { hoch) }\end{array}$ & 4.67 & 4.68 & 260.500 & 491.500 & -.056 & .955 & $\begin{array}{c}0.478 \\
>0.05\end{array}$ \\
\hline
\end{tabular}

Die Auswertung der Items über die Funktion von Sprachlernspielen weist eben auf keinen statistischen signifikanten Unterschied von zwei Semestern hin.

Tabelle 37: Ergebnisse von MWU-Tests zum Vergleich der Funktionen der Sprachlernspiele

\begin{tabular}{|c|c|c|c|c|c|c|c|}
\hline \multirow{2}{*}{$\begin{array}{l}\text { Stimmen Sie } \\
\text { folgenden } \\
\text { Aussagen zu? } \\
1 \text { gar nicht- } 4 \text { völlig } \\
\text { Sprachlernspiele... }\end{array}$} & \multicolumn{2}{|c|}{ Mittelwert } & \multirow[t]{2}{*}{$\begin{array}{c}\text { Mann- } \\
\text { Whitney-U }\end{array}$} & \multirow[t]{2}{*}{$\begin{array}{l}\text { Wilcoxon } \\
\text { W }\end{array}$} & \multirow[t]{2}{*}{ Z } & \multirow{2}{*}{$\begin{array}{l}\text { Asymp. } \\
\text { Sig. } \\
\text { (zweiteilig) }\end{array}$} & \multirow{2}{*}{$\begin{array}{c}\text { P. } \\
\left(=\begin{array}{l}\text { Sig. } / \\
\text { 2) }\end{array}\right.\end{array}$} \\
\hline & WS & SS & & & & & \\
\hline $\begin{array}{l}\text { 3.1...machen } \\
\text { Grammatiklernen } \\
\text { interessanter. }\end{array}$ & 3.24 & 3.20 & 255.000 & 580.000 & -.198 & .843 & $\begin{array}{l}0.4215 \\
>0.05\end{array}$ \\
\hline
\end{tabular}


Tabelle 37: Fortsetzung

\begin{tabular}{|c|c|c|c|c|c|c|c|}
\hline \multirow{2}{*}{$\begin{array}{l}\text { Stimmen Sie } \\
\text { folgenden } \\
\text { Aussagen zu? } \\
1 \text { gar nicht- } 4 \text { völlig } \\
\text { Sprachlernspiele... }\end{array}$} & \multicolumn{2}{|c|}{ Mittelwert } & \multirow[t]{2}{*}{$\begin{array}{c}\text { Mann- } \\
\text { Whitney-U }\end{array}$} & \multirow[t]{2}{*}{$\begin{array}{c}\text { Wilcoxon } \\
\text { W }\end{array}$} & \multirow[t]{2}{*}{$\mathrm{Z}$} & \multirow{2}{*}{$\begin{array}{l}\text { Asymp. } \\
\text { Sig. } \\
\text { (zweiteilig) }\end{array}$} & \multirow{2}{*}{$\begin{array}{l}\text { P. } \\
(=\text { Sig./ } \\
\text { 2) }\end{array}$} \\
\hline & WS & $\overline{\text { SS }}$ & & & & & \\
\hline $\begin{array}{l}3.2 \ldots \text { machen } \\
\text { Wortschatzlernen } \\
\text { interessanter. }\end{array}$ & 3.14 & 3.00 & 239.000 & 564.000 & -.569 & .570 & $\begin{array}{l}0.285 \\
>0.05\end{array}$ \\
\hline $\begin{array}{l}\text { 3.3....bauen Angst } \\
\text { beim Sprechen ab. }\end{array}$ & 3.1 & 3.08 & 258.000 & 489.000 & -.112 & .910 & $\begin{array}{l}0.455 \\
>0.05\end{array}$ \\
\hline $\begin{array}{l}\text { 3.4...erhöhen meine } \\
\text { Sprechzeit auf Deutsch } \\
\text { im Unterricht. }\end{array}$ & 3.14 & 3.24 & 243.000 & 474.000 & -.547 & .584 & $\begin{array}{l}0.292 \\
>0.05\end{array}$ \\
\hline $\begin{array}{l}\text { 3.5...erhöhen meine } \\
\text { sprachliche Fähigkeit } \\
\text { im allgemein }\end{array}$ & 3.05 & 3.04 & 261.500 & 586.500 & -.025 & .980 & $\begin{array}{l}0.490 \\
>0.05\end{array}$ \\
\hline $\begin{array}{l}\text { 3.6...verbessern die } \\
\text { Kommunikation } \\
\text { zwischen mir und den } \\
\text { Kommilitonen }\end{array}$ & 3.29 & 3.36 & 240.000 & 471.000 & -.590 & .555 & $\begin{array}{l}0.2775 \\
>0.05\end{array}$ \\
\hline $\begin{array}{l}3.7 \ldots \text {...geben mir die } \\
\text { Chance, im Unterricht } \\
\text { Zeit fürs Regelfinden zu } \\
\text { haben und autonom zu } \\
\text { denken. }\end{array}$ & 3.1 & 3.00 & 249.000 & 574.000 & -.377 & .707 & $\begin{array}{l}0.3535 \\
>0.05\end{array}$ \\
\hline $\begin{array}{l}\text { 3.8...erhöhen die } \\
\text { Autonomie beim } \\
\text { Sprachlernen. }\end{array}$ & 2.81 & 3.12 & 199.500 & 430.500 & -1.538 & .124 & $\begin{array}{l}0.062 \\
>0.05\end{array}$ \\
\hline $\begin{array}{l}\text { 3.9... erhöhen die } \\
\text { Konzentration im } \\
\text { Unterricht. }\end{array}$ & 3.29 & 3.24 & 259.500 & 584.500 & -.077 & .939 & $\begin{array}{l}0.4695 \\
>0.05\end{array}$ \\
\hline $\begin{array}{l}3.10 \ldots \text { bringen } \\
\text { Abwechslung in den } \\
\text { Unterricht. }\end{array}$ & 3.62 & 3.48 & 226.000 & 551.000 & -.933 & .351 & $\begin{array}{l}0.1755 \\
>0.05\end{array}$ \\
\hline $\begin{array}{l}3.11 \ldots \text { sind hilfreich für } \\
\text { Fremdsprachenlernen } \\
\text { im Allgemeinen. }\end{array}$ & 3.38 & 3.40 & 257.500 & 488.500 & -.130 & .896 & $\begin{array}{l}0.448 \\
>0.05\end{array}$ \\
\hline $\begin{array}{l}3.12 \ldots \text { sind interessant, } \\
\text { aber für mich etwas zu } \\
\text { schwer. }\end{array}$ & 2.29 & 2.56 & 208.500 & 439.500 & -1.293 & .196 & $\begin{array}{l}0.098 \\
>0.05\end{array}$ \\
\hline $\begin{array}{l}3.13 \ldots \text { sind } \\
\text { Zeitverschwendung im } \\
\text { Unterricht. }\end{array}$ & 1.48 & 1.32 & 216.000 & 541.000 & -1.219 & .223 & $\begin{array}{l}0.1115 \\
>0.05\end{array}$ \\
\hline
\end{tabular}


Gemäß den statistischen Ergebnissen kann man feststellen, dass die Lernenden von der Effizienz von Sprachlernspielen fest überzeugt sind, wie schon im WS 2016-17. Sie sind der Meinung, dass Sprachlernspiele ihre sprachliche Kompetenz verbessern, Angst abbauen, autonomes Lernen fördern, und Interaktion im Unterricht erhöhen können. Sie sind der Ansicht, dass Sprachlernspiele den Unterricht interessanter machen und keine Zeitverschwendung sind. Ihre Meinungen zum Einsatz der Sprachlernspiele bleiben unverändert im Vergleich zum vorhergehenden Semester.

Die Ergebnisse von Item 6 zeigen, dass die Mehrheit der Meinung ist, dass Sprachlernspiele $30 \%$ bis $70 \%$ der Unterrichtszeit einnehmen sollten. Die erwünschte Spielzeit ist im Vergleich zu der im WS 2016-17 kürzer (siehe Kapitel 5.1.4). Sie sind der Meinung, dass das Erlernen der deutschen Sprache mit Sprachlernspielen interessanter und entspannter ist. Sprachlernspiele helfen ihnen, sich im Unterricht zu konzentrieren und das Gelernte besser im Kopf zu behalten.

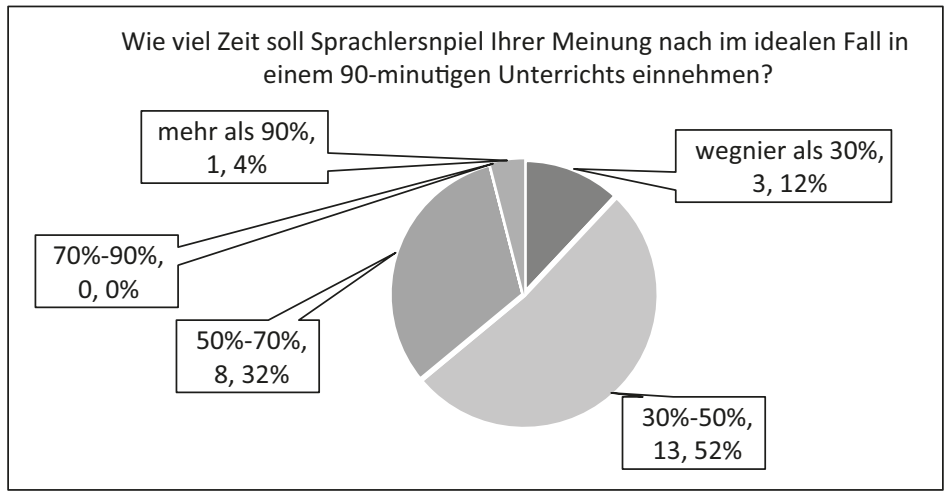

Abbildung 14: Die Ergebnisse von Item 6 der Befragung zum Einsatz der Sprachlernspiele SS 2017

Durch Item 7 kann man feststellen, dass die Mehrheit der Studierenden (96\%) findet, dass die Einsatzzeit der Sprachlernspiele im SS 2017 angemessen ist, nur eine Person davon ist der Meinung, dass der Spieleinsatz zu viel Zeit nimmt: 


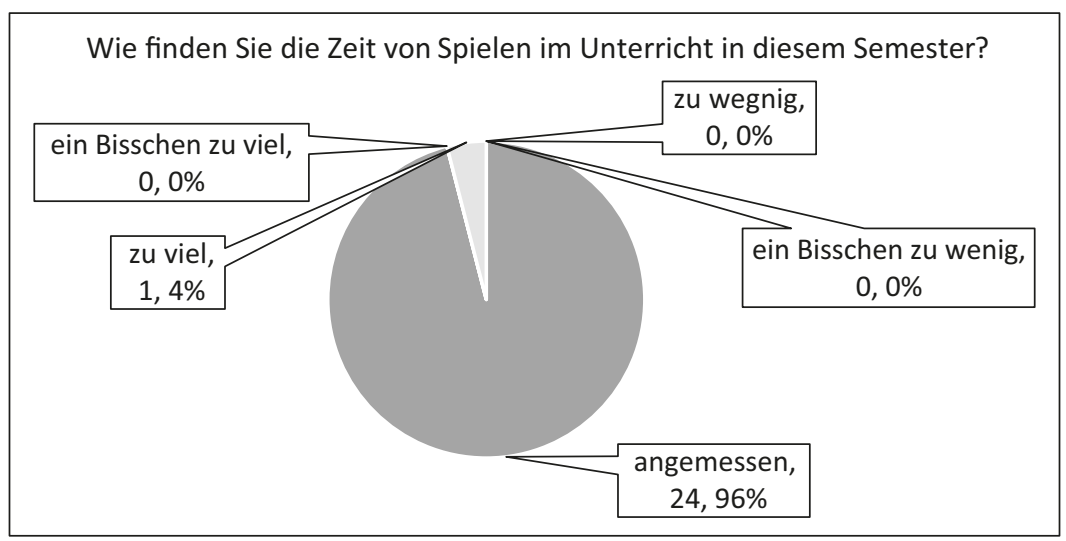

Abbildung 15: Ergebnisse von Item 7 der Befragung zum Einsatz der Sprachlernspiele SS 2017

Die Ergebnisse von Item 8 und Item 12 (siehe Tabelle 38) zeigen, dass die Mehrheit der Lernenden einen Unterricht mit Sprachlernspielen bevorzugen. Es gibt einen Studenten, der geschrieben hat, dass Sprachlernspiele nicht so gut wie traditioneller Unterricht funktionieren, weil Sprachlernspiele das Lernen nicht so gut wie die traditionelle Methode fördern können, trotzdem will er Sprachlernspiele im kommenden Semester noch haben, nur nicht so oft wie im SS 2017.

Tabelle 38: Übersicht der Ergebnisse von Item 8 und 12

\begin{tabular}{lcc}
\hline Item & $\begin{array}{c}\text { Unterricht ohne } \\
\text { Sprachlernspiel }\end{array}$ & $\begin{array}{c}\text { Unterricht mit } \\
\text { Sprachlernspiel }\end{array}$ \\
\hline 8. Welche Unterrichtsform gefällt Ihnen besser? & 1 & 24 \\
12. Hoffen Sie, dass Sprachlernspiele im nächsten & 0 & 25 \\
Semester weiter benutzt werden? & & \\
\hline
\end{tabular}

Zur Begründung ihrer Zuneigung zum Sprachlernspiel haben die Lernenden zahlreiche Kommentare geschrieben, die in 6 Kategorien gegliedert werden können. 
Tabelle 39: Übersicht der Kommentare von Item 10 und 12

Warum bevorzuge ich Sprachlernspiele?

Das Lernen und der Unterricht werden interessanter und abwechslungsreicher.

Es hilft dem Erlernen der Sprache. Ich kann mir das

Gelernte leichter merken.

Es ist motivierend.

Die Atmosphäre im Unterricht ist besser und ich habe mehr Kommunikation mit anderen.

Es erhöht die Konzentration im Unterricht.

Ich mag es einfach, ohne Grund.
Zahl der zugehörigen

Meinungen

17

15

7

4

4

4

Ein Befragter hat noch dazu geschrieben, dass diese Methode sehr gut und viel besser als die traditionelle Methode aus der Oberschule ist. Über ihre Erfahrung mit Sprachlernspielen vor dem Studium meinen die meisten, dass es Sprachlernspiele ja gab, aber sehr selten eingesetzt wurden (siehe Abbildung 16).

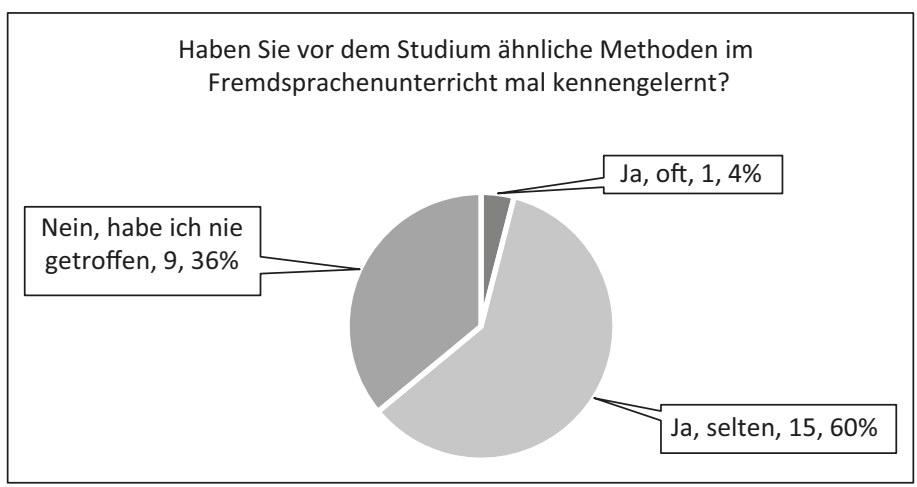

Abbildung 16: Ergebnisse von Item 11 der Befragung zum Einsatz der Sprachlernspiele SS 2017

Zusammenfassend lässt sich sagen, dass die Studierenden Sprachlernspiele als gute Methode ansehen. Die diskutierten Effekte von Sprachlernspielen werden von ihnen wahrgenommen, wie die Befragungsergebnisse im WS 2016-17 auch gezeigt haben. Alle möchten in der Zukunft weiter mit dieser Methode lernen, weil es interessant und motivierend ist und beim Erlernen der deutschen Sprache Hilfe leisten kann. 
Während die Effizienz des Sprachlernspiels hinsichtlich des Lernfortschritts nur teilweise bestätigt werden kann, zeigen die subjektive Erfahrung der Lernenden eine deutlich positive Bewertung gegenüber dem Sprachlernspiel wie schon im vorhergehenden Semester. Da die persönliche Sichtweise für die vorliegende explorativ-interpretative Forschung von Bedeutung ist (vgl. Caspari et al. 2007, S. 499f.), wird nicht nur die Meinung der Studierenden untersucht, sondern auch die Sicht der Lehrenden. Im SS 2017 werden 7 Lehrerinnen zum Unterricht eingeladen, um den Unterrichtsprozess zu beobachten und den Unterricht zu evaluieren. Ihre Beobachtungen und Evaluationen werden als wichtige Ergänzung zu einem möglichst vollständigen Bild vom Einsatz der Sprachlernspiele betrachtet, die im folgenden Unterkapitel ausgewertet und analysiert werden sollen.

\subsubsection{Auswertung und Analyse zu den Ergebnissen von Unterrichtsbeobachtungen im Sommersemester 2017}

Unterrichtsbeobachtung wird als eine wichtige empirische Methode zur Datenerhebung im DaF-Unterricht häufig angewendet (vgl. Brede 2014, S. 137; Caspari et al. 2007, S. 501). Helmke (2017, S. 292) nimmt Beobachtung als „Königsweg zur Beschreibung und Bewertung des Unterrichts“ und notwendige Datenerhebungsmethode für Unterrichtsforschung an.

Da die Untersuchung des Fremdsprachenunterrichts heutzutage sich nicht nur auf Ergebnis und Leistung des Fremdsprachenlernens, sondern auch auf den Prozess des Erlernens konzentriert, wird das Geschehen im Unterricht unter die Lupe genommen. Man versucht herauszufinden, was im Unterricht passiert sowie warum und wie das Erlernen einer Fremdsprache davon geprägt werden kann (vgl. Liu 2016, S. 157). Brede (2014, S. 144) vertritt die Meinung, dass die Ergebnisse von der beobachteten Situation abhängen, und deswegen keine von der Praxis losgelöste Forschung erfolgen kann. Sie verdeutlicht, dass die Analyseergebnisse damit nicht auf statistische Kennwerte beschränkt sind, sondern an Beispielen sichtbar gemacht werden. Sie sind anschaulich und können praxisrelevante Erkenntnisse bieten.

Dieser Vorteil von Unterrichtsbeobachtung ist für die vorliegende Forschung von großer Bedeutung. An dieser Stelle soll geklärt werden, wie der Unterrichtsverlauf für andere Lehrpersonen aussieht. Mit Hilfe dieser Methode kann bestätigt werden, ob Sprachlernspiele beobachtbare Veränderungen in den Unterricht aus Lehrer-Perspektive bringen können.

In der vorliegenden Forschung werden mehrere offene, nicht-teilnehmende Fremdbeobachtungen in natürlicher Situation bzw. im Klassenraum 
durchgeführt. Sieben Lehrerinnen ${ }^{26}$ werden zum beliebigen Unterricht eingeladen. Jede Lehrerin soll eine Stunde mit gleichem Lerninhalt von zwei Gruppen hospitieren, so dass sie die Sitzungen vergleichen kann. Sie dürfen ein beliebiges Stundenpaar auswählen. Insgesamt werden 4 Sitzungspaare hospitiert, weil einige Lehrerinnen zu zweit zu einem Sitzungspaar gekommen sind.

Die Lehrerinnen bekommen vor der Stunde ein Set von Beobachtungsbögen, die anhand der Unterrichtsbeobachtungsbogen 12 und 13 aus der Fernstudieneinheit „Unterrichtsbeobachtung und Lehrerverhalten“ (Ziebell und Meese 2004, S. 165-167) für die vorliegende Forschung adaptiert sind.

Das Fragenbogen-Set (siehe Anhang 11) besteht aus folgenden Beobachtungsbögen: Das Verlaufsprotokoll (Beobachtungsbogen A) dient dem Zweck, den Beobachtenden die Sozial- und Arbeitsformen bewusst zu machen; Befragungen zur Evaluation einzelner Stunden (Beobachtungsbogen B) für die Bewertung des Unterrichts; Befragung zum Vergleich der Stunden von zwei Gruppen mit offenen Fragen (Beobachtungsbogen C).

Den Beobachtungsbogen A füllen die Beobachterinnen während der Beobachtung aus. Der Bobachtungsbogen B soll nach der Beobachtung jeder einzelnen Sitzung sofort ausgefüllt werden. Der Beobachtungsbogen $C$ wird nach der Beobachtung von beiden Stunden ausgefüllt. Die Lehrerinnen können also den Beobachtungsbogen $\mathrm{C}$ ausarbeiten und ihre Gedanken ausführlich formulieren. Alle Beobachtungsbögen werden auf Chinesisch formuliert und ausgefüllt, weil die Mehrheit der Beobachtenden kein Deutsch beherrscht. Da der Unterricht mehrheitlich auf Chinesisch organisiert wird, können alle den Unterrichtsverlauf verstehen.

Hier werden der Beobachtungsbogen B mit Ratingskala statistisch analysiert, um herauszufinden, ob sich die Sitzungen mit gleichem Inhalt und unterschiedlicher Methode von Perspektiven der Beobachtenden unterscheiden.

Folgende Hypothesen werden gestellt:

1. Es gibt beobachtbare Unterschiede zwischen beiden Gruppen.

2. Die EG weist deutlich mehr Interaktion auf als die KG.

3. Die EG scheint mehr motiviert zu sein als die KG.

4. Die Sitzung der EG wird besser als die der KG von den Beobachtenden bewertet.

26 Eine Deutsch-, Eine Fränzösisch-, eine Japanisch- und vier Englisch-Lehrerinnen werden eingeladen. Es gibt insgesamt nur 2 Deutsch-Lehrerinnen an der TU Kunming. Da die Mehrheit des Unterrichts auf Chinesisch durchgeführt wird, sind alle eingeladenen Lehrerinnen in der Lage, den Unterricht zu verstehen und den Prozess zu dokumentieren. 
Der Beobachtungsbogen B enthält folgende Kriterien:

Tabelle 40: Überblick vom Beobachtungsbogen B

Kriterien

Zum Unterrichtsdesign und zur Vorbereitung des

Unterrichts

Zur Durchführung des Unterrichts

Zur Effizienz des Unterrichts

Zur Bewertung des Unterrichts
Operationalisiert in den Items

Nr.

$1-9$

$10-27$

$28-40$

41

Durch MWU-Test mit SPSS kann man Unterschiede bei Item 5, 6 und 7 zum Design und zur Vorbereitung des Unterrichts feststellen (siehe Anhang 12).

Tabelle 41: Items mit signifikantem Unterschied zu Unterrichtsdesign und Vorbereitung

Item Nr. Aussagen zu Unterrichtsdesign und Vorbereitung (1 ich stimme völlig nicht $\mathrm{zu}-4$ ich stimmt völlig zu)

5 Es gibt genügend Zeit für anwendungsorientierte Unterrichtsaktivitäten.

6 Es gibt genügend Gelegenheit für $S .^{27}$, zusammenzuarbeiten

7 Es gibt keine Gelegenheit für S., durch Tun zu lernen.

Tabelle 42: Übersicht der Ergebnisse von MWU-Tests Item 5,6 und 7

\begin{tabular}{|c|c|c|c|c|c|c|c|}
\hline \multirow[t]{2}{*}{ Item Nr. } & \multicolumn{2}{|c|}{ Mittelwert } & \multirow{2}{*}{$\begin{array}{c}\text { Mann- } \\
\text { Whitney-U }\end{array}$} & \multirow{2}{*}{$\begin{array}{c}\text { Wilcoxon } \\
\text { W }\end{array}$} & \multirow[t]{2}{*}{$\mathrm{Z}$} & \multirow{2}{*}{$\begin{array}{l}\text { Asymp. Sig. } \\
\left(2^{\star} \text { einteilig }\right)\end{array}$} & \multirow{2}{*}{$\begin{array}{c}\text { P. } \\
\text { (= Sig./ 2) }\end{array}$} \\
\hline & EG & KG & & & & & \\
\hline 5 & 4.0000 & 2.5714 & 7.000 & 35.000 & -2.622 & $.026^{\mathrm{b}}$ & $0.013<0.05^{\star}$ \\
\hline 6 & 3.8571 & 1.8571 & .500 & 28. & -3.238 & $.001^{\mathrm{b}}$ & $0.005<0.01^{*}$ \\
\hline 7 & 1.0000 & 2.1429 & 10.500 & 38.500 & -2.245 & $.073^{\mathrm{b}}$ & $0.037<0.05^{\star}$ \\
\hline
\end{tabular}

Die statistischen Ergebnisse zeigen:

Bei Item 5 kann man einen signifikanten Unterschied zwischen den Gruppen beobachten. Die Beobachtenden merken an, dass die EG deutlich mehr Zeit für anwendungsorientierte Aktivitäten im Unterricht als die KG hat.

27 „S.“ steht in diesem Kapitel als Abkürzung für Studierende. 
Item 6 deutet auf einen sehr signifikanten Unterschied zwischen beiden Gruppen hin: Die Lernenden in der EG arbeiten mehr als die in der KG zusammen.

Item 7 zeigt Unterschiede beim handelnden Lernen. Die Lernenden in der EG können durch Tun die Sprache lernen, während die von der KG weniger Chance zum handelnden Lernen haben.

Zum Verlauf des Unterrichts weisen Item 14, 15, 18, 19, 20, 21 und 27 Unterschiede hin:

Tabelle 43: Items mit signifikantem Unterschied zum Verlauf des Unterrichts

\begin{tabular}{cl}
\hline Item Nr. & $\begin{array}{l}\text { Aussagen zur Durchführung des Unterrichts } \\
\text { (1 ich stimme völlig nicht zu } \mathbf{- 4} \text { ich stimmt völlig zu) }\end{array}$ \\
\hline 14 & Es wird eine Reihe von vielfältigen Aktivitäten organisiert. \\
15 & Das Lehrerverhalten unterstützt die aktive Teilnahme von S. \\
18 & Die Lernmaterialien bieten den S. vielfältige Möglichkeiten zu lernen. \\
19 & Die Lernmaterialien sind kreativ, interessant und motivierend. \\
20 & Die Lernmaterialien enthalten spielerische Elemente. \\
21 & Die Mehrheit des Unterrichts ist lehrer-dominiert. \\
27 & Die Interaktivität zwischen S. ist gut und zeitlich angemessen. \\
\hline
\end{tabular}

Tabelle 44: Übersicht der Ergebnisse der MWU-Tests von Items zum Verlauf des Unterrichts

\begin{tabular}{|c|c|c|c|c|c|c|c|}
\hline \multirow[t]{2}{*}{ Item Nr. } & \multicolumn{2}{|c|}{ Mittelwert } & \multirow{2}{*}{$\begin{array}{c}\text { Mann- } \\
\text { Whitney-U }\end{array}$} & \multirow{2}{*}{$\begin{array}{c}\text { Wilcoxon } \\
\text { W }\end{array}$} & \multirow[t]{2}{*}{$\mathbf{Z}$} & \multirow{2}{*}{$\begin{array}{l}\text { Asymp. Sig. } \\
\left(2^{\star} \text { einteilig) }\right.\end{array}$} & \multirow{2}{*}{$\begin{array}{c}\mathbf{P} \\
(=\text { Sig./ } / 2)\end{array}$} \\
\hline & EG & KG & & & & & \\
\hline 14 & 3.7143 & 2.1429 & 5.500 & 33.500 & -2.554 & $.011^{\mathrm{b}}$ & $0.0055<0.01^{\star}$ \\
\hline 15 & 3 & 2.85 & & 37.500 & -2.152 & .0 & $0.0265<$ \\
\hline 18 & 3.7143 & 2.4286 & & 0 & -1.994 & $.073^{\mathrm{b}}$ & $0.037<0.05^{*}$ \\
\hline 19 & 3.8571 & 2.4286 & 8.500 & 36 & -2.273 & $.038^{\mathrm{b}}$ & $0.019<0.05^{\star}$ \\
\hline 20 & 3.8571 & 1.4286 & 5 & 28.500 & -3.275 & $.001^{\mathrm{b}}$ & $0.0005<0.001^{\star}$ \\
\hline 21 & 1.8571 & 3.7143 & 4.500 & 32.500 & -2.689 & $.007^{\mathrm{b}}$ & $0.0035<0.01^{\star}$ \\
\hline 27 & 3.5714 & 2.0000 & 6.500 & 34.500 & -2.394 & .017 & $0.0085<0.01^{\star}$ \\
\hline
\end{tabular}

Bei Item 14, 20, 21 und 27 deuten die statistischen Ergebnisse auf einen sehr signifikanten Unterschied hin. Das verdeutlicht, dass die Aktivitäten in der EG in den Augen der Beobachtenden abwechslungsreicher und spielerischer als die in der KG gestaltet sind. Der Unterricht der KG ist eher lehrer-dominierend, 
während der von der EG lernerzentriert ist. Die Interaktivität unter Studierenden in der EG ist deutlich höher als die in der KG.

Item 15, 18 und 19 zeigen ebenso einen statistisch signifikanten Unterschied und beweisen, dass das Lehrverhalten und die Lernmaterialien in EG-Stunden den Lernenden vielfältigere, interessantere und motivierendere Möglichkeiten fürs Lernen anbieten.

Zur Effizienz des Unterrichts zeigen folgende Items statistische Unterschiede:

Tabelle 45: Items mit signifikantem Unterschied zur Effizienz des Unterrichts

\begin{tabular}{ll}
\hline Item Nr. & $\begin{array}{l}\text { Aussagen zur Effizienz des Unterrichts } \\
\text { (1 ich stimme völlig nicht zu - 4 ich stimmt völlig zu) }\end{array}$ \\
\hline 29 & Die Mehrheit der S. scheint im Unterricht konzentriert zu sein. \\
30 & Die Mehrheit der S. nimmt an Aktivitäten aktiv teil. \\
31 & Die Mehrheit der S. spricht nicht gern. \\
34 & Die Mehrheit der S. arbeitet aktiv miteinander und löst die Aufgaben gemeinsam. \\
35 & Die Mehrheit der S. scheint motiviert fürs Fremdsprachenlernen zu sein. \\
38 & Der Unterricht läuft sehr aktiv. \\
39 & Die Atmosphäre des Unterrichts ist sehr lebhaft. \\
\hline
\end{tabular}

Tabelle 46: Übersicht der Ergebnisseder MWU-Tests von Items zur Effizienz des Unterrichts

\begin{tabular}{|c|c|c|c|c|c|c|c|}
\hline \multirow[t]{2}{*}{ Item Nr. } & \multicolumn{2}{|c|}{ Mittelwert } & \multirow{2}{*}{$\begin{array}{c}\text { Mann- } \\
\text { Whitney-U } \\
\end{array}$} & \multirow{2}{*}{$\begin{array}{c}\text { Wilcoxon } \\
\text { W }\end{array}$} & \multirow[t]{2}{*}{$\mathrm{Z}$} & \multirow{2}{*}{$\begin{array}{l}\text { Asymp. Sig. } \\
\text { (2*einteilig) }\end{array}$} & \multirow{2}{*}{$\begin{array}{c}\mathbf{P} \\
(=\text { Sig./2) }\end{array}$} \\
\hline & EG & KG & & & & & \\
\hline 29 & 3.7143 & 2.8571 & 11.000 & 39.000 & -1.875 & $.097^{\mathrm{b}}$ & $0.0485<0.05^{\star}$ \\
\hline 30 & 3.8571 & 2.8571 & 6.000 & 34.000 & -2.593 & $.017^{\mathrm{b}}$ & $0.0085<0.01^{*}$ \\
\hline 31 & 1.4286 & 2.4286 & 3.000 & 31.000 & -2.912 & $.004^{\mathrm{b}}$ & $0.002<0.01^{\star}$ \\
\hline 34 & 3.5714 & 2.1429 & 3.000 & 31.000 & -2.877 & $.004^{\mathrm{b}}$ & $0.002<0.01^{\star}$ \\
\hline 35 & 3.4286 & 1.8571 & 4.000 & 32.000 & -2.756 & $.007^{\mathrm{b}}$ & $0.0035<0.01^{\star}$ \\
\hline 38 & 3.7143 & 2.2857 & 7.500 & 35.500 & -2.328 & $.026^{\mathrm{b}}$ & $0.013<0.05^{\star}$ \\
\hline 39 & 3.8571 & 2.2857 & 5.000 & 33.000 & -2.681 & $.011^{\mathrm{b}}$ & $0.0055<0.01^{\star}$ \\
\hline
\end{tabular}

Die Ergebnisse von Item 30, 32, 34, 35 und 39 zeigen den sehr signifikanten Unterschied zwischen beiden Gruppen und beweisen, dass sich die Mehrheit der Studierenden in der EG im Unterricht aktiver und motivierter verhält und mehr Bereitschaft zum Sprechen und zur Zusammenarbeit mit anderen zeigt. Die Atmosphäre im Unterricht der EG ist lebhafter als die der KG. 
Item 29 und 38 deuten auch einen statistisch signifikanten Unterschied an. Das bedeutet, dass die Studierenden in der EG mehr Konzentration im Unterricht zeigen und der Unterricht der EG aktiver als der von der KG läuft.

Item 41 ermöglicht den Beobachtenden den Unterricht zu bewerten. Bei diesem Item wird ebenso der statistisch signifikante Unterschied bestätigt. Nach der Meinung der Beobachtenden kann der Unterricht von der EG besser als der von der KG gesehen werden:

Tabelle 47: Übersicht der Ergebnisse von MWU-Test von Item 41

\begin{tabular}{|c|c|c|c|c|c|c|c|}
\hline \multirow{2}{*}{$\begin{array}{l}\text { 41. Bitte } \\
\text { bewerten Sie } \\
\text { den Unterricht } \\
\text { (1 sehr schlecht- } \\
5 \text { sehr gut) } \\
\end{array}$} & \multicolumn{2}{|c|}{ Mittelwert } & \multirow{2}{*}{$\begin{array}{c}\text { Mann- } \\
\text {-Whitney-U }\end{array}$} & \multirow{2}{*}{$\begin{array}{c}\text { Wilcoxon } \\
\text { W }\end{array}$} & \multirow[t]{2}{*}{$\mathrm{Z}$} & \multirow{2}{*}{$\begin{array}{l}\text { Asymp. Sig. } \\
\left(2^{\star} \text { einteilig) }\right.\end{array}$} & \multirow{2}{*}{$\begin{array}{c}\mathbf{P} \\
(=\text { Sig./ } \\
2)\end{array}$} \\
\hline & EG & KG & & & & & \\
\hline & 4.7143 & 3.7143 & 7.500 & 35.500 & -2.328 & $.026^{\mathrm{b}}$ & $\begin{array}{l}0.013 \\
<0.05^{\star}\end{array}$ \\
\hline
\end{tabular}

Unter Berücksichtigung der statistischen Ergebnisse können wir annehmen, dass die gestellten Hypothesen bestätigt werden können. Die zwei Gruppen weisen deutliche Unterschiede auf. Die Studierenden der EG zeigen im Unterricht mehr Interaktion und Motivation als die der KG. Der Unterricht der EG wird auch besser als der von der KG bewertet.

Zusammenfassend lässt sich sagen, dass die eingeladenen Lehrerinnen Unterschiede hinsichtlich Interaktion, Motivation und Lehrmethode zwischen zwei Gruppen feststellen können und den Unterricht von der EG als positiver wahrnehmen. Die Antworten zu den offenen Fragen aus Beobachtungsbogen C (siehe Anhang 20) bestätigen eben diese Stellungnahme:

Die Lehrerinnen sind der Meinung, dass die Stunden mit Sprachlernspielen im Vergleich zu den Stunden ohne spielerische Elemente deutlich motivierender wirken und besser sind. Die lebhafte Atmosphäre im Unterricht wird von allen Beobachtenden als wichtiges Merkmal gesehen. Während der Hospitation merken sie deutliche Unterschiede der beiden Gruppen bei der Bereitschaft zum Sprechen, zum Zusammenarbeiten, zum Teilnehmen an verschiedenen Lernaktivitäten und zur Beantwortung der Fragen. Die Studierenden der EG scheinen konzentrierter, motivierter und kommunikationsfreudiger zu sein als die der KG. Die Sitzungen mit Sprachlernspielen sind interessanter und lernerzentriert gestaltet. Man kann in den Stunden durch Tun in einer Gruppe lernen, statt nur 
zuzuhören. Die Mehrheit der Beobachtenden meint, dass sie die spielerische Methode in der Zukunft auch im eigenen Unterricht einsetzen wollen.

\subsubsection{Schlussfolgerung der Forschungsphase Sommersemester 2017}

Die Befragung zu Lernmotivation und Unterrichtsbewertung von der Lernenden-Seite zeigen keinen großen Unterschied zwischen beiden Gruppen, sowohl zur eigenen Sprachlernfähigkeit, als auch zur Unterrichtsbewertung. Eine positive Änderung der Einstellung gegenüber der Sprache der EG kann durch den Unterschied von Item 1.3 von den zweien Semestern bestätigt werden. Auch die höhere Interaktivität im Unterricht mit Sprachlernspielen wird bestätigt.

Zum Lernfortschritt zeigen die Ergebnisse der Semesterprüfung nur einen statistischen Unterschied bei der Note vom Hörverstehen. Die Auswertung von dem letzten C-Test spricht dafür, dass die EG eine deutlich bessere Leistung am Ende des Semesters erbringt.

Der teilweise bestätigte bessere Lernfortschritt der EG spricht dafür, dass die Lernenden von Sprachlernspielen positiv beeinflusst werden. Diese Stellungnahme wird auch von der Befragung zum Sprachlernspiel bestätigt. Die Lernenden sind der Meinung, dass das Sprachlernspiel eine interessante und motivierende Unterrichtsmethode ist. Sie zeigen hohes Interesse und hohe Bereitschaft, mit dieser Methode weiterzuarbeiten. Die hohe Interaktivität durch den Einsatz von Sprachlernspielen wird auch von den Lernenden wahrgenommen. Die Leistungsdifferenzierung innerhalb der EG zeigt allerdings, dass der Wirkungsgrad der neuen Methode bei den Lernenden variieren kann.

Die Ergebnisse der Unterrichtsbeobachtung verdeutlichen die positiven Effekte von Sprachlernspielen. Die Beobachtenden sind einverstanden, dass Sprachlernspiele eine gute Unterrichtsatmosphäre schaffen können sowie positive Lerneffekte und motivierende Funktion haben. Die Sitzungen mit Sprachlernspielen werden aus der Lehrer-Perspektive deutlich besser als die ohne Spiel bewertet.

Da die bisherige Analyse hauptsächlich aus Sicht der Lernenden erfolgt, wird im folgenden Unterkapitel die Sicht der Lehrperson einbezogen, um die Effizienz des Spieleinsatzes auf Motivation und Lernfortschritt von der LehrpersonPerspektive näher zu betrachten.

\subsection{Auswertung und Analyse zum Lehrtagebuch vom Studienjahr 2016-2017}

In der vorliegenden Forschung wird ein Lehrtagebuch als wichtiges Dokumentationsinstrument für die gesamte Forschung eingesetzt. In zwei Semestern 
werden nach jedem Unterrichtstag basierend auf den Didaktisierungsplänen dokumentiert, wie die verschiedenen Aktivitäten in beiden Gruppen zeitlich und inhaltlich gelaufen sind. Außerdem gibt es einen zusammenfassenden Ablaufkommentar der Lehrperson, um wichtige Ereignisse und im Unterricht beobachtbares Lernerverhalten sowie Unterrichtserlebnisse der Lehrperson zu notieren.

Die Daten aus dem Lehrtagebuch sollen analysiert werden, um potenzielle Unterschiede zwischen zwei Gruppen bei der Durchführung der Sprachlernspiele im Unterricht herauszufinden. Außerdem soll die Analyse des Lehrtagebuchs Erhellung zur Spielorganisation und dem Unterrichtserlebnis lehrerseits bringen: Die zeitliche Notierung ermöglicht eine statistische Analyse der eingesetzten Sprachlernspiele, um festzustellen, ob Übungen in Spielform zeitlich anders als originale Übungen sind (siehe Kapitel 6.1). In diesem Unterkapitel wird der tägliche Kommentar der Lehrperson qualitativ analysiert, um mögliche Merkmale und Tendenzen von zwei Gruppen aus Sicht der Lehrperson zusammenzufassen. Da die Stunden von beiden Gruppen immer am gleichen Tag hintereinander stattfanden, sind große Emotions- und Gesundheitsschwankungen der Lehrperson ausgeschlossen.

Vor der Analyse des Lehrtagebuchs werden die Funktionen und Merkmale des Lehrtagebuchs als Forschungsinstrument hier kurz dargestellt.

Das Tagebuch wird von Altrichter (1998, S. 26) als „eines der wichtigsten Werkzeuge" einer forschenden Lehrperson angesehen. McDonough (1994, S. 58) weist nach, dass es drei Hauptherangehensweisen mit dem Tagebuch im Fremdsprachenunterricht gibt:

1. Lernertagebuch mit pädagogischem Ziel als Kommunikations- und Protokollinstrument zwischen Lernenden und Lehrenden

2. Lernertagebuch als Forschungsgegenstand zur Erforschung des Sprachlernsprozesses

3. Tagebuch als Lehrerfortbildungsinstrument

Er pointiert, dass das Lehrtagebuch relativ selten geführt wird, obwohl das Lehrtagebuch ein Forschungsinstrument mit viel Potenzial ist. Er zitiert von Elliott (1991), dass ein Lehrtagebuch „observations, feelings, reactions, interpretations, reflections, hunches, hypotheses, explanations" aufzeichnet.

Liu (2016, S. 185-188) schließt sich dieser Meinung an, dass das Lehrtagebuch eine ausführliche Untersuchungsgeschichte darstellen kann und ein Schatzkammer von Daten ist. Er deutet an, dass das Wichtigste des Lehrertagbuchs darin liegt, dass es ermöglicht, die sich immer wieder ändernden Emotionen, 
Ansichten und Einstellungen der Lehrperson während des Lehrens zu veranschaulichen.

Das Lehrtagebuch soll trotz seiner kritisch zu sehenden Subjektivität und mangelnden Validität als eine Forschungsmethode eingesetzt und mit anderen Datenerhebungsmethoden zusammen als Triangulation eingesetzt werden (vgl. Liu 2016, S. 187; Gass und Mackey 2012, S. 183). Liu (2016, S. 186) erörtert, dass das Tagebuch als Forschungsinstrument seit fast 30 Jahren in der Fremdsprachen-Unterrichtsforschung im Ausland etabliert ist, aber in China nur selten gebraucht wird. Für Aktionsforschung wie in der vorliegenden Arbeit spielt das Lehrtagebuch eine besondere Rolle. Boeckmann (2016, S. 594) teilt die Ansicht und erörtert, dass das Lehrtagebuch ein jederzeit verwendbares, organisatorisch unaufwendiges Forschungsinstrument ist, und als zentrale Forschungsdokumentation angesehen werden sollte. Er zitiert vom Altrichter und Porsch (2007, S. 30f.), dass das Lehrtagebuch „die Entwicklung der Vorstellungen und Einsichten über die verschiedenen Phasen des Forschungsprozesses hinweg" dokumentieren kann.

An dieser Stelle soll auch betont werden, dass die subjektiven Unterrichtserlebnisse der Lehrperson für die vorliegende Forschung von großer Bedeutung sind. Die Lehrenden nehmen am Unterricht aktiv teil und erleben den Unterricht mit den Lernenden zusammen. Erst seit den letzten 20 Jahren interessiert sich die Fremdsprachendidaktik für die Rolle der Lehrkräfte im unterrichtlichen Geschehen (vgl. Caspari 2016, S. 306). Im Vergleich zur Rolle der Lernenden gerät die Rolle des Fremdsprachenlehrers in den Hintergrund der Sprachlernforschung (vgl. Königs 2017, S. 323f.). Caspari (2016, S. 306) demonstriert, dass Unterricht kein Prozess-Produkt-Konzept ist, sondern ein von vielfältigen Wechselbeziehungen sowie Wert- und Interessenkonflikten geprägtes, komplexes und damit potenziell unvorhersehbares Geschehen. Die Lehrperson ist in erster Linie für Veränderungen des Unterrichts verantwortlich. Davon ausgehend sollen Emotionen und Unterrichtserlebnisse der Lehrperson nicht außer Acht gelassen werden.

In der englischsprachigen Sprachlehrforschung wird der Emotion der Lehrenden in den letzten 20 Jahren immer mehr Beachtung geschenkt (vgl. Agudo, Juan de Dios Martinez 2018, S. 2; Yin und Lee 2012, S. 56). Lehren und Lernen sind mit Emotionen eng verbunden und sollen als affektiver Prozess gesehen werden (vgl. Yin und Lee 2012, S. 56-58; Hargreaves 2000, S. 812; O'Connor 2008, S. 118). Agudo (2018, S. 2) deutet an, dass die enge Verknüpfung von Emotion und Kognition im Unterrichtsprozess dazu führen kann, dass die Kognition und Emotion der Lehrperson ihre Lehrmethoden stark prägen können. Dewaeles (2018, S. 126) weist nach, dass die Freude der Lernenden im Unterricht stark 
von den Lehrenden abhängt. Cuéllar und Oxford (2018, S. 58f.) verweisen darauf, dass Lehrende und Lernende ihre eigenen Emotionen, Erfahrungen und Erwartungen ins Klassenzimmer bringen und alle diese Faktoren miteinander vermischen. Deswegen ist die emotionale Reaktion von Lehrenden auf das Unterrichtsgeschehen von großer Bedeutung. Die Emotionen von Lernenden und Lehrenden haben gegenseitigen Einfluss. Die Emotionen von Lernenden können eine bedeutende Rolle bei der Unterrichtsmethode sowie bei der Zufriedenheit und Motivation der Lehrenden spielen (vgl. Smith und King 2018, 325). Deswegen dürfen die persönliche Unterrichtsbetrachtung und -bewertung mit den Emotionen und der Einstellung der Lehrperson nicht als irrelevante Forschungsdaten gesehen werden. Das subjektive Unterrichtserlebnis der Lehrperson widerspiegelt das Unterrichtsgeschehen und soll als sinnvolle Interpretation der Unterrichtssituation aus Lehrerperspektive betrachtet werden.

Anschließend werden die Ablaufnotizen und Unterrichtszusammenfassungen der Lehrperson über die zwei Semester analysiert, um die Unterrichtsbeobachtung der Lehrperson zu rekonstruieren. Mit der Rekonstruktion wird das Unterrichtsgeschehen aus der Lehrerperspektive herauskristallisiert. Eine quantitative Analyse wird vorgenommen, so dass die einzelnen inhaltlichen Merkmale der Texte mit Hilfe eines Kategoriensystems gemessen werden. Die Kategorien werden aus der theoretischen Vorüberlegung bezüglich des Unterrichtsgeschehens abgeleitet und während der Durchsicht der Materialien verfeinert und erweitert.

Der tägliche Beitrag im Lehrtagebuch enthält Informationen zur Raumbedingung des Unterrichts an jedem Tag, Kommentare über den Ablauf des Spiels, eine vergleichende Zusammenfassung über Unterrichtsgeschehen der zwei Gruppen und Reflexionen der Lehrperson zum Unterricht. Die Stunden der zwei Gruppen finden in beiden Semestern immer nacheinander statt, insgesamt 2 Tage pro Woche, an jedem Tag jeweils 2 US für jede Gruppe. Im WS 2016-17 werden 23 und im SS 201724 Lehrbuchbeiträge handschriftlich aufgeschrieben.

Die quantitative Inhaltsanalyse dient den Aussagen und Kommentaren über das Unterrichtsgeschehen. Nach dem gründlichen Lesen allen Beiträge werden die Textsegmente nach den inhaltlichen Merkmalen kodiert. Im Zuge der Kodierung und fallübergreifenden Auswertung ließen sich die Textsegmente in vier zentrale Kategorien herausarbeiten: 1. Zum Unterrichtsverhalten und Lernklima (UV); 2. Zur Interaktionssituation und sprachlichen Produktion im Unterricht (IS); 3. Zum Lehrprozess (LP) und 4.Wichtige Reflexion (WR). Ein Beispiel für die Kodierung von Textstellen sowie ein Tagbucheintrag findet man im Anhang 16. In den unteren Tabellen ist die Anzahl der Textsegmente unter den genannten Kategorien und den einzelnen Codes in den zwei Semestern dargestellt. 
Tabelle 48: Kodierung von Textsegmenten des Lehrtagebuchs WS 2016-17

\begin{tabular}{|c|c|c|c|}
\hline \multirow{3}{*}{$\begin{array}{l}\text { Kategorie } \\
\text { Unterrichtsverhalten } \\
\text { und Lernklima (UV) }\end{array}$} & \multirow{2}{*}{$\begin{array}{c}\text { Code } \\
\text { EG besser als KG (UV1) }\end{array}$} & \multicolumn{2}{|c|}{$\begin{array}{c}\text { Anzahl der } \\
\text { Textsegmente }\end{array}$} \\
\hline & & \multicolumn{2}{|c|}{7} \\
\hline & KG besser als EG (UV2) & \multicolumn{2}{|c|}{6} \\
\hline & & KG & EG \\
\hline & Hohe Konzentration (UV3) & 1 & 5 \\
\hline & Niedrige Konzentration (UV4) & 1 & 1 \\
\hline & Aktive Teilnahme (UV5) & 2 & 6 \\
\hline & Inaktive Teilnahme (UV6) & 2 & 1 \\
\hline & Gutes Lernklima (UV7) & 0 & 6 \\
\hline & Schlechtes Lernklima (UV8) & 4 & 0 \\
\hline \multirow{6}{*}{$\begin{array}{l}\text { Interaktionssituation } \\
\text { und sprachliche } \\
\text { Produktion (IS) }\end{array}$} & Häufige Interaktion (IS1) & 0 & 8 \\
\hline & Wenig Interaktion (IS2) & 5 & 0 \\
\hline & Viel Sprachproduktion (IS3) & 0 & 2 \\
\hline & Wenig Sprachproduktion (IS4) & 0 & 0 \\
\hline & Gute Aussprache (IS 5) & 0 & 0 \\
\hline & Schlechte Aussprache (IS 6) & 0 & 0 \\
\hline \multirow{4}{*}{ Lehrprozess (LP) } & Gelungene Organisation (LP1) & 0 & 17 \\
\hline & Ungelungene Organisation (LP2) & 4 & 16 \\
\hline & EG besser als KG (LP3) & \multicolumn{2}{|c|}{2} \\
\hline & KG besser als EG (LP4) & \multicolumn{2}{|c|}{2} \\
\hline \multicolumn{2}{|c|}{ Wichtige Reflexion (WR) } & \multicolumn{2}{|c|}{25} \\
\hline
\end{tabular}

Tabelle 49: Kodierung von Textsegmenten des Lehrtagebuchs SS 2017

\begin{tabular}{llcc}
\hline Kategorie & Code & \multicolumn{2}{c}{$\begin{array}{c}\text { Anzahl der } \\
\text { Textsegmente }\end{array}$} \\
\hline Unterrichtsverhalten & EG besser als KG (UV1) & \multicolumn{2}{c}{5} \\
und Lernklima (UV) & KG besser als EG (UV2) & \multicolumn{2}{c}{1} \\
& & KG & EG \\
\cline { 2 - 4 } & Hohe Konzentration (UV3) & 5 & 7 \\
& Niedrige Konzentration (UV4) & 4 & 2 \\
& Aktive Teilnahme (UV5) & 3 & 15 \\
& Inaktive Teilnahme (UV6) & 7 & 2 \\
& Gutes Lernklima (UV7) & 0 & 15 \\
& Schlechtes Lernklima (UV8) & 8 & 1
\end{tabular}


Tabelle 49: Fortsetzung

\begin{tabular}{llcc}
\hline Kategorie & Code & \multicolumn{2}{c}{$\begin{array}{c}\text { Anzahl der } \\
\text { Textsegmente }\end{array}$} \\
\hline \multirow{3}{*}{ Interaktionssituation } & & KG & EG \\
\cline { 3 - 4 } (IS) & Häufige Interaktion (IS1) & 0 & 12 \\
& Wenig Interaktion (IS2) & 4 & 0 \\
& Viel Sprachproduktion (IS3) & 0 & 3 \\
& Wenig Sprachproduktion (IS4) & 2 & 0 \\
& Gute Aussprache (IS5) & 0 & 4 \\
Lehrprozess (LP) & Schlechte Aussprache (IS 6) & 1 & 0 \\
& Gelungene Organisation (LP1) & 0 & 10 \\
& Ungelungene Organisation (LP2) & 0 & 4 \\
\multicolumn{1}{l}{ Wichtige Reflexion (WR) } & 0 & 0 \\
\hline
\end{tabular}

Betrachtet man die Kategorie Unterrichtsverhalten und Lernklima, können erkennbare Unterschiede zwischen beiden Semestern festgestellt werden. In dem ersten Semester zeigen zwei Gruppen hinsichtlich Unterrichtsverhalten keinen deutlichen Unterschied, während in dem zweiten Semester die EG viel besser als die KG bewertet wird. In beiden Semestern wird das Lernklima der EG positiver bewertet (siehe UV7), während das der KG negativ bewertet wird (siehe UV8). Beide Gruppen unterscheiden sich im Hinblick auf Interaktion eben sehr. Studierende der EG haben mehr Interaktion im Unterricht und äußern sich mehr (siehe IS1und IS3). Die Aussprache der EG wird auch positiver als die von der KG bewertet (siehe IS5 und IS6).

Die Ergebnisse der Analyse veranschaulichen, dass ab dem zweiten Semester, aus der Lehrperson-Perspektive gesehen, die Studierenden der EG ein deutlich positiveres Bild als die der KG zeigen. Die Lernenden der EG sind im Unterricht aktiver, kooperationsfreudiger und (aus)sprachlich besser. Wie weiter oben schon diskutiert wurde, spiegelt die subjektive Interpretation der Lehrperson einen Teil der Unterrichtsrealität wider. Die Wahrnehmung der Lehrperson beeinflusst den Studierenden und die von den Studierenden vice versa. Die positiven und negativen Bewertungen gegenüber der Lerngruppe, wenn auch subjektiv, haben Konsequenzen für den Unterricht. Die Ergebnisse zeigen, dass die Lehrperson mit der Gruppe mit Spieleinsatz deutlich zufriedener ist, als mit der Gruppe ohne Spiel. Die spielerischen Gruppen- und Partnerarbeiten bringen aus 
Lehrer-Perspektive mehr Interaktion und Kommunikationsmöglichkeit in den Unterricht und hilft der Lehrenden, ein gutes Lernklima zu gestalten.

Infolgedessen lässt sich zusammenfassen, dass höhere Motivation und bessere sprachliche Kompetenz bei der EG durch die Analyse des Lehrtagebuchs bestätigt werden können.

\subsection{Zwischenfazit}

Die vorliegende Forschung versucht, die Effizienz von Sprachlernspielen in DaFUnterricht in China zu überprüfen. Mehrere Instrumente werden eingesetzt und mehrere Datenquellen werden zu verschiedenen Zeitpunkten erhoben, um möglichst viele Facetten des Untersuchungsgegenstandes zu erfassen, den Aussagewert der Daten zu erhöhen, und die Konsistenz der erhaltenen Information zu überprüfen. Der Überblick der eingesetzten Datenerhebungsmethoden sowie gewonnenen Daten in den zwei Semestern werden in folgenden Tabellen dargestellt.

Tabelle 50: Überblick der eingesetzten Datenerhebungsmethoden und der gewonnenen Daten WS 2016-17

\begin{tabular}{|c|c|c|c|}
\hline $\begin{array}{l}\text { Datenerhebungs- } \\
\text { methode }\end{array}$ & Gruppe & Zeit & Daten \\
\hline $\begin{array}{l}\text { Befragung zur } \\
\text { Lernerbiographie }\end{array}$ & \multirow{4}{*}{$\begin{array}{l}\text { EG und } \\
\text { KG }\end{array}$} & 3. Woche & $\begin{array}{l}\text { Persönliche Daten (Geschlecht, Alter } \\
\text { und Herkunft) } \\
\text { Lernbiographie } \\
\text { Lernerfahrung und Lernmotivation } \\
\text { gegenüber Fremdsprachenlernen }\end{array}$ \\
\hline $\begin{array}{l}\text { Befragung zur } \\
\text { Lernmotivation }\end{array}$ & & \begin{tabular}{|l|} 
12. Dez. 2016 \\
14. Woche
\end{tabular} & $\begin{array}{l}\text { Lernmotivation } \\
\text { Einstellung gegenüber Deutsch } \\
\text { Unterrichtsbewertung }\end{array}$ \\
\hline Semesterprüfung & & 18. Woche & \ Leistung von zwei Klausuren \\
\hline Lehrtagebuch & & $\begin{array}{l}\text { jede Stunde ab } \\
\text { 5. Woche }\end{array}$ & $\begin{array}{l}\text { Verlauf einzelner Stunden } \\
\text { Zeit einzelner Aktivitäten } \\
\text { Verlauf der eingesetzten Spiele (EG) } \\
\text { Vergleich zweier Gruppen }\end{array}$ \\
\hline $\begin{array}{l}\text { Befragung zum } \\
\text { Einsatz des } \\
\text { Sprachlernspiels }\end{array}$ & EG & $\begin{array}{l}\text { 12. Dez. } 2016 \\
\text { 14. Woche }\end{array}$ & $\begin{array}{l}\text { Bewertung zur Methode } \\
\text { Sprachlernspiele im Allgemeinen } \\
\text { Bewertung vom einzelnen Spiel }\end{array}$ \\
\hline
\end{tabular}


Tabelle 51: Überblick der eingesetzten Datenerhebungsmethoden und der gewonnenen Daten SS $2017^{28}$

\begin{tabular}{|c|c|c|c|}
\hline Datenerhebungsmethode & Gruppe & Zeit & Daten \\
\hline $\begin{array}{l}\text { Befragung zur } \\
\text { Lernmotivation }\end{array}$ & \multirow{5}{*}{$\begin{array}{l}\text { EG und } \\
\text { KG }\end{array}$} & $\begin{array}{l}\text { 2. Juni } \\
\text { 14. Woche }\end{array}$ & $\begin{array}{l}\text { Lernmotivation } \\
\text { Einstellung gegenüber Deutsch } \\
\text { Unterrichtsbewertung }\end{array}$ \\
\hline C-Tests ${ }^{*}$ & & \begin{tabular}{|l|} 
Nach jeder \\
Einheit \\
\end{tabular} & $\diamond$ Note von einzelnen C-Tests \\
\hline Semesterprüfung & & 18. Woche & $\bullet$ Leistung von zwei Klausuren \\
\hline Lehrtagebuch & & jede Stunde & $\begin{array}{l}\text { Verlauf einzelner Stunden } \\
\text { Zeit einzelner Aktivitäten } \\
\text { Verlauf der eingesetzten Spiele (EG) }\end{array}$ \\
\hline Unterrichtsbeobachtung" & & $\begin{array}{l}\text { im Mai } \\
\text { und Juni } \\
2017\end{array}$ & $\begin{array}{l}\text { Unterrichtsverlauf } \\
\text { Bewertung der einzelnen Stunde } \\
\text { Vergleich der zwei Stunden } \\
\text { Vergleich zweier Gruppen }\end{array}$ \\
\hline $\begin{array}{l}\text { Befragung zum Einsatz } \\
\text { des Sprachlernspiels }\end{array}$ & EG & 15. Woche & $\begin{array}{l}\text { Bewertung zur Methode } \\
\text { Sprachlernspiele im Allgemeinen } \\
\text { Bewertung einzelner Spiele }\end{array}$ \\
\hline
\end{tabular}

Die im Kapitel 4.2.1 gestellten Forschungsfragen können jetzt beantwortet werden:

Tabelle 52: Antworten der Forschungsfragen zur Motivationserhöhung

\section{Forschungsfragen zur Motivationserhöhung}

1. Können die Lernenden mehr Selbstvertrauen beim Sprachlernen durch Einsatz von Sprachlernspielen gewinnen?

2. Verbessert sich die Einstellung der Lernenden gegenüber der Zielsprache bzw. Deutsch durch Sprachlernspiele?

3. Wird der Unterricht mit Sprachlernspielen besser als der Unterricht ohne Sprachlernspiele bewertet?
Ja. Weil sie sich im Unterricht mehr äußern, deutlich mehr Bereitschaft zur Kommunikation haben und zusammenarbeiten können.

Ja. Einstellungsänderungen gegenüber der eigenen Sprachlernfähigkeit (WS 2016-17) und der Zielsprache (SS 2017) werden innerhalb der Gruppen festgestellt, obwohl kein statistischer Unterschied zwischen den zwei Gruppen festgestellt werden kann.

Die Studierenden haben beide Unterrichtstypen als sehr gut bewertet, während die hospitierenden Lehrenden und die Lehrperson die Sitzungen mit Sprachlernpielen deutlich besser bewertet haben.

28 Die mit " gekennzeichnete Methode gibt es im WS 2016-17 nicht. 
Tabelle 52: Fortsetzung

\section{Forschungsfragen zur Motivationserhöhung}

4. Sind die Lernenden im

Unterricht mit Sprachlernspielen aktiver als die im Unterricht ohne Sprachlernspiele?
Ja. Die Lernenden sind der Meinung, dass die Interaktion im Unterricht mit Spielen sehr hoch ist, und dass sie sehr viel mit ihren Kommilitonen zusammenarbeiten können. Die hospitierenden Lehrenden und die Lehrperson sind auch davon überzeugt, dass die Lernenden in Sitzungen mit Sprachlernspielen deutlich mehr Bereitschaft zu Interaktion, Kommunikation und Zusammenarbeit zeigen.

Tabelle 53: Antworten der Forschungsfragen zur Verbesserung der sprachlichen Kompetenzen

\section{Forschungsfragen zur Verbesserung der sprachlichen Kompetenzen}

1. Können die Lernenden mit Sprachlernspielen in den durchgeführten Prüfungen und Tests bessere Leistungen als die Lernenden ohne Sprachlernspiel erreichen?

2. Können Sprachlernspiele bestimmte Fertigkeiten stärker fördern, beispielsweise eine bessere Fertigkeit im Hören?
Teilweise ja. Die Studierenden aus der EG zeigen deutlich bessere Leistungen bei einem C-Test im SS 2017 und bei zwei Teilfertigkeiten in zwei Semestern.

Teilweise ja. Bei der Teilfertigkeit Schreiben im WS 2016-17 und der Teilfertigkeit Hörverstehen im SS 2017 haben die Studierenden aus der EG statistisch höhere Noten bekommen.

Zwei Hypothesen werden gestellt:

1. Lernende, die den DaF-Unterricht mit Sprachlernspielen besuchen, sind in einer vergleichbaren Situation signifikant mehr motiviert, als Lernende in einem lehrerzentrierten DaF-Unterricht ohne Sprachlernspiele.

2. Lernende, die den DaF-Unterricht mit Sprachlernspielen besuchen, entwickeln in einer vergleichbaren Situation bessere sprachliche Kompetenzen, als Lernende in einem lehrerzentrierten DaF-Unterricht ohne Sprachlernspiele.

Die Hypothese 1 wird mit Hilfe der umfassenden Daten überprüft und mehrheitlich durch statistische Analysen bestätigt, während die Hypothese 2 anhand der Analyse nur teilweise bestätigt werden kann.

Unter Berücksichtigung der statistischen Ergebnisse der Unterrichtsbeobachtung können wir annehmen, dass ein lernerzentrierter Unterricht mit Sprachlernspielen motivierender ist. Die EG zeigt einen deutlichen Motivationszuwachs 
im WS 2016-17 und eine verbesserte Einstellung gegenüber der Zielsprache im SS 2017. Die verbesserte Kommunikationsbereitschaft von der EG, die durch Befragung der Lernenden, Unterrichtsbeobachtung von hospitierenden Lehrenden sowie durch das Lehrtagebuch mehrfach bestätigt wird, zeigt, dass die EG kommunikationsfreudiger und aktiver als die KG ist.

Die Lernenden entwickeln mit Hilfe des Sprachlernspiels eine höhere Bereitschaft zur Kommunikation und zum Mitarbeiten im Unterricht, zeigen hohe Interaktion und Konzentration im Unterricht und fühlen sich von einer interessanten und entspannten Unterrichtsatmosphäre umgegeben. Die Lernenden wollen sich in dieser entspannten lebhaften Atmosphäre äußern und mit anderen zusammenarbeiten.

Während beide Klassen ihre jeweilige Unterrichtsform als gut bewerten, sind die hospitierenden Lehrenden und die Lehrperson der Meinung, dass der Unterricht mit Spielen deutlich besser als der traditionelle Unterricht wirkt. Der Grund der Meinungsdiskrepanz liegt wahrscheinlich darin, dass die Lernenden der KG keine Erfahrung mit lernerzentriertem Unterricht mit Sprachlernspielen haben und deswegen nicht wissen, dass Fremdsprachenunterricht anders verlaufen kann, während die hospitierenden Lehrenden und die Lehrperson zwei Unterrichtssituationen erlebten und daher die Unterschiede erkennen konnten.

Die übergreifende Forschungsfrage:

Können Lernende aus einer Lerntradition, die stark von der lehrerzentrierten Unterrichtsform und passivem Lernen geprägt ist, sich an interaktive/kommunikative Methoden wie das Sprachlernspiel gewöhnen, und dabei bessere Leistungen und höhere Motivation als im lehrerzentrierten Unterricht zeigen?

kann wie im Folgenden beantwortet werden:

Die Methode mit Sprachlernspielen kann bei einer Zielgruppe mit langer lehrerzentrierter Lernerfahrung effizient eingesetzt werden. Die Lernenden können sich an interaktive/kommunikative Methoden wie Sprachlernspiele gewöhnen und teilweise bessere Leistungen als im lehrerzentrierten Unterricht erreichen.

Hier muss betont werden, dass in dieser Forschung besserer Lernfortschritt nicht nur eine bessere Note in den Prüfungen bedeutet. Die teilweise bestätigte bessere Prüfungsleistung der EG ist nur ein Teil des Erfolgs des Sprachlernspieleinsatzes. Vielmehr haben die Sprachlernspiele zur Änderung des Lernerlebnisses sowohl der Lernenden als auch der Lehrenden beigetragen. Die Unterrichte mit Sprachlernspielen wirken interessanter und abwechslungsreicher, helfen Beziehung aufzubauen und einander kennenzulernen. Die Ergebnisse der Befragungen zum Sprachlernspiel der EG aus beiden Semestern bestätigen dies und 
belegen, dass die Lernenden Spaß im Unterricht haben und diese Methode willkommen heißen. Das Lehrtagebuch bestätigt ebenfalls, dass die Lehrperson von der Methode Sprachlernspiel überzeugt ist und den Unterricht mit Sprachlernspielen positiver bewertet. Die hospitierenden Lehrenden vertreten diese Meinung ebenfalls.

Die hohe Bereitschaft zur Zusammenarbeit und Interaktion in der EG zeigt eine erhöhte Motivation der Lernenden, obwohl sie die eigene Sprachlernfähigkeit nicht besser bewerten. Sprachlernspiele helfen dabei, ein angstfreies und förderndes Unterrichtsklima zu bauen, sodass die Lernenden sich im Unterricht wohl fühlen und höhere WTC bekommen.

Im Großen und Ganzen wird bewiesen, dass das Sprachlernspiel eine effiziente Unterrichtsmethode ist, auch wenn die Lerngruppe lange Lernerfahrung mit lehrerzentriertem Fremdsprachenunterricht hatte. Nach einer Zeit der Anpassung zeigt ein interaktiver Unterricht mit Sprachlernspielen seine motivierende Funktion, insbesondere bei Kommunikation und aktiver Teilnahme am Unterricht.

Betrachtet man die C-Test-Ergebnisse im SS 2017 (siehe Kapitel 5.2.4) kann festgestellt werden, dass der Unterschied zwischen beiden Gruppen im Laufe der Zeit immer größer geworden ist. Daher kann man vermuten, dass die Wirkung von Sprachlernspielen mit der Zeit sichtbarer wird. Einerseits zeigt die Leistungsdifferenzierung innerhalb der EG in zwei Semestern, dass die Lernenden der EG eine Gewöhnungsphase für die spielerische Methode brauchen. Andererseits kann die Neugier für die neue Sprache am Anfang das Lernen fördern, weil die Lernenden beider Gruppen Anfänger sind. Dieses Motiv kann allerdings langsam verschwinden. Im Vergleich zum traditionellen Unterricht kann Unterricht mit Sprachlernspielen das Interesse und die Motivation fürs Sprachlernen besser fördern und hat daher eine nachhaltigere Wirkung. Daher ist es für die vorliegende Forschung von Bedeutung, den Lernfortschritt der Studierenden in einer längeren Phase zu beobachten.

Nach der Aktionsforschung werden die beiden Klassen von einer Kollegin der Forscherin übergenommen. Das Erlernen der deutschen Sprache wird im Studienjahr 2017-18 für ein zweites Jahr fortgesetzt. Beide Gruppen werden von der Kollegin gleich behandelt. Das heißt, dass der einzige Unterschied beider Gruppen darin liegt, dass die EG Erfahrung mit Sprachlernspielen hat. Die Unterrichtssituation von beiden Gruppen in dem zweiten Jahr sind daher im vergleichbaren Kontext und deren Leistungen in Semesterprüfungen können für die vorliegende Forschung weitere Informationen anbieten.

Nach der Meinung der Kollegin zeigen die beiden Gruppen unter gleichem Kontext verschiedene Unterrichtsverhalten und Lernfortschritte, insbesondere bei der Kommunikation und der Aussprache. Im Folgenden wird die Leistung 
von Semesterprüfungen im WS 2017-18 und SS 2018 durch SPSS mit WMUTest analysiert, um den möglichen Unterschied festzustellen ${ }^{29}$.

Da die EG im ersten Studienjahr mehr Kommunikationsbereitschaft und höhere Aktivität zeigt, und in dem letzten C-Test im SS 2017 bessere Leistung erreicht, kann man vermuten, dass die EG mehr Möglichkeit zum Üben und Verwendung der Zielsprache bekommen hat und mehr Bereitschaft zum autonomen Lernen besitzt. Infolgedessen wird die folgende Hypothese aufgestellt:

Die Prüfungsleistung des Studienjahres 2017-18 der EG ist besser als die der KG.

Wegen zu vieler Fehlzeiten werden manche Studierende nicht zu den Prüfungen zugelassen, deswegen variiert die Anzahl der Teilnehmenden zu verschiedenen Prüfungen.

Die statistischen Ergebnisse zeigen, dass die Hypothese bei Klausur 2 im WS 2017-18 und Klausur 1 in beiden Semestern bestätigt wird. Darunter zeigt Klausur 1 im Wintersemester einen hoch signifikanten Unterschied, Klausur 2 im Wintersemester auch einen sehr signifikanten Unterschied. Das heißt, dass die EG deutlich bessere Leistungen als die KG im zweiten Studienjahr erbracht hat, besonders bei der Klausur 1 für Hörverstehen, Schreiben und Übersetzen.

Tabelle 54: Ergebnisse von MWU- Tests der Semesterprüfungen im Studienjahr 2017-18

\begin{tabular}{|c|c|c|c|c|c|c|c|}
\hline & \multicolumn{2}{|c|}{ Mittelwert } & $\begin{array}{c}\text { Mann- } \\
\text { Whitney-U }\end{array}$ & $\begin{array}{c}\text { Wilcoxon } \\
\text { W }\end{array}$ & $\mathrm{Z}$ & $\begin{array}{l}\text { Asymp. Sig. } \\
\text { (zweiteilig) }\end{array}$ & $\begin{array}{c}\text { P. } \\
\text { (= Sig./ 2) }\end{array}$ \\
\hline \multicolumn{8}{|c|}{ WS 2017-18 } \\
\hline & EG(25) & KG(33) & & & & & \\
\hline $\begin{array}{c}\text { Klausur } \\
1\end{array}$ & $83.4400^{*}$ & 67.7879 & 213.500 & 774.500 & -3.128 & .002 & $\begin{array}{c}0.001 \\
\leq 0.001^{\star}\end{array}$ \\
\hline $\begin{array}{c}\text { Klausur } \\
2\end{array}$ & $78.5000^{*}$ & 66.2727 & 258.500 & 819.500 & -2.419 & .016 & $\begin{array}{c}0.008 \\
\leq 0.01^{\star}\end{array}$ \\
\hline \multicolumn{8}{|c|}{ SS 2018} \\
\hline & EG (21) & KG (31) & & & & & \\
\hline \multirow[t]{2}{*}{$\begin{array}{c}\text { Klausur } \\
1\end{array}$} & $68.4048^{*}$ & 57.0484 & 209.000 & 705.000 & -2.173 & .030 & $\begin{array}{c}0.015 \\
\leq 0.05^{\star}\end{array}$ \\
\hline & EG25) & KG (30) & & & & & \\
\hline $\begin{array}{c}\text { Klausur } \\
2\end{array}$ & $65.9600^{*}$ & 60.2167 & 295.500 & 760.500 & -1.344 & .179 & $\begin{array}{l}0.0895 \\
>0.05\end{array}$ \\
\hline
\end{tabular}

29 Rohdaten dazu siehe Anhang 21. 
Die verzögerte Wirkung lässt sich wie folgt erklären:

Erstens ist Sprachlernen ein Prozess, der in verschiedenen „Erwerbsstufen“ abläuft. Der Erwerbsprozess ist nicht linear. Wenn die Lernenden weder genügende Motivation noch Interesse haben, kommt es zur „Fossilisierung des Spracherwerbs auf einer unteren Erwerbsstufe" (vgl. Roche 2013, S. 125-127). Es lässt sich vermuten, dass die EG mit Hilfe von Sprachlernspielen mehr motiviert für den Spracherwerb als die KG ist, und deswegen weiter vorankommen kann, während die KG auf einer unteren Stufe bleibt.

Zweitens konnten die Effekte der Sprachlernspiele im ersten Studienjahr wegen der Anzahl der Stichproben nicht statistisch bestätigt werden. Da der Fremdsprachenerwerb von vielen Faktoren beeinflusst wird und eine Methode nur zusammen mit anderen Lernfaktoren wirken kann, ist der Effekt einer Methode nicht besonders stark. Je kleiner ein Effekt ist, desto größer muss der Stichprobenumfang sein (vgl. Krekeler 2011, S. 213). Döring und Bortz (2016, S. 843) machen deutlich, dass ein kleiner Effekt erst mit 310 bis 393 Probanden bestätigt werden kann. Nur ein großer Effekt kann mit 20 bis 26 Probanden gezeigt werden. Davon ausgehend kann man vermuten, dass die Effekte von Sprachlernspielen im ersten Jahr nur kleine Effekte im Vergleich mit dem traditionellen Unterricht zeigen. Die Wirkung wird aber im zweiten Jahr größer und kann deswegen mit der kleinen Probandengruppe bestätigt werden.

Zusammenfassend lässt sich sagen, dass sich die Wirksamkeit von Sprachlernspielen auf Leistungsebene erst in einer längeren Phase zeigen lässt. Im ersten Studienjahr zeigt die Mehrheit der Prüfungen keinen deutlichen Unterschied, während große Unterschiede im folgenden Studienjahr zu beobachten sind. Die Implikationen dieses Resultats sind vielfältig:

Einerseits kann man sagen, dass Sprachlernspiele für die sprachliche Kompetenz der Lernenden einen wichtigen Beitrag leisten können. Sprachlernspiele fördern besonders die kommunikative Kompetenz, wie Hören, Schreiben und Aussprache. Die bessere Leistung von Teilfertigkeiten im ersten Studienjahr und die von Klausur 1 im zweiten Studienjahr können diese Wirkung beweisen. Auch im Unterrichtsverlauf wird die bessere Aussprache von Lernenden der EG von der Lehrperson bestätigt.

Andererseits muss man zugeben, dass Sprachlernspiele für einen Deutschunterricht, der stark prüfungsorientiert ist und in kurzer Zeit Erfolg bringen soll, als ungeeignet und ineffizient betrachtet werden können. Obwohl Sprachlernspiele keine schlechtere Leistung im ersten Jahr verursachen, ist der Einsatz des Sprachlernspiels organisatorisch nicht ökonomisch, weil die Vorbereitung und Durchführung sehr aufwendig sind (siehe Kapitel 6). Da man mit traditionellem Unterricht in kurzem Zeitraum einen ähnlichen Lernfortschritt erreichen kann, 
ist der Einsatz von Sprachlernspielen nicht besonders sinnvoll. Für die Zielgruppe der vorliegenden Forschung, die schwachen Fremdsprachenlernenden mit geringer Leistungsorientierung und schlechter Lernerfahrung im Fremdsprachenunterricht, können Sprachlernspiele in einem langzeitigen Kursangebot für gute sprachliche Kompetenz und positive Lernerfahrung viel leisten. Das spricht dafür, dass die Methodenwahl dem Lernziel und Unterrichtstyp entsprechend vorsichtig getroffen werden muss.

Man kann also zu dem Schluss kommen, dass das Sprachlernspiel eine effiziente Unterrichtsmethode im Fremdsprachenunterricht in China sein kann. Diese Methode hat motivierende Wirkung, kann das Erlernen der Zielsprache fördern und hilft den Lehrenden, einen Fremdsprachenunterricht interaktiv/ kommunikativ und motivierend zu gestalten. Der Unterricht mit Sprachlernspielen ist in einer vergleichbaren Unterrichtssituation (mit denselben Unterrichtsmaterialien, derselben Unterrichtszeit und identischem Unterrichtsinhalt) effizienter als der Unterricht ohne Einsatz von Sprachlernspielen. Diese Effizienz zeigt sich aber erst nach einer relativ langen Phase. Erstens weil die Lernenden Zeit brauchen, um sich an eine neue lernerzentrierte interaktive Methode anzupassen. Zweitens werden die Vorteile vom Sprachlernspiel gegenüber der traditionellen Methode erst nach relativ langer Zeit sichtbar.

Die vorliegende Forschung hat auch Implikationen für die Praxis. Es wird gezeigt, dass man Sprachlernspiele in großer Gruppe von Lernenden mit lehrerzentrierter Lernerfahrung gut einsetzen kann. Im folgenden Schritt des Projekts wird die Methode der Adaption von traditionellen Übungen auf Sprachlernspiele vermittelt, die für den Großgruppenunterricht geeignet sind. Eine weitere Analyse des Lehrtagebuchs veranschaulicht die Durchführung des Spiels. Außerdem werden die Befragungen zum Einsatz des Spiels lernerseits vertieft untersucht, um herauszufinden, ob bestimmte Spieletypen von Lernenden besonders bevorzugt werden und wenn ja, warum. 


\section{Empirische Untersuchung Teil II: Praxisorientierte Anweisung vom Einsatz der Sprachlernspiele im DaF-Unterricht in China}

Dieses Kapitel widmet sich der Praxis vom Spieleinsatz im DaF-Unterricht in China. Folgende Forschungsfragen sollen damit beantwortet werden:

1. Kann der Unterricht mit Sprachlernspielen zeitlich genau so effizient sein wie Unterricht ohne Sprachlernspiele?

2. Kann man traditionelle Übungsformen als Sprachlernspiele adaptieren?

3. Kann man Sprachlernspiele in einer größeren Gruppe einsetzen, auch wenn die Rahmenbedingungen nicht ideal für den Spieleinsatz sind?

\subsection{Effiziente Planung und Durchführung von spielerischer Gruppenarbeit im Großgruppenunterricht}

Die didaktischen und methodischen Prinzipien bezüglich eines Spieleinsatzes in der Großgruppe werden hier anhand der Forschungsergebnisse diskutiert und erklärt, sodass Fremdsprachenlehrende mit Hilfe der vorliegenden Forschung ihren Unterricht mit Großgruppen in China oder in anderen Teilen der Welt spielerisch gestalten können. Die Vorteile der Gruppenarbeit für Großgruppenunterricht wurden im Kapitel 2.2.3 ausführlich diskutiert. Da Sprachlernspiele in der Aktionsforschung mehrheitlich in der Sozialform „Gruppenarbeit“ durchgeführt werden, wird an dieser Stelle auf die effiziente Planung und Organisation von Gruppenarbeiten näher eingegangen.

Die Lehrperson muss wissen, dass jede teilnehmeraktivierende Arbeitsform am Anfang mehr Zeit braucht. Für Lernende, die keine oder wenig Erfahrung mit Gruppen- und Partnerarbeit haben, ist eine systematische Einübung notwendig. Mit der Einübungsphase werden der Ablauf und das Ziel des Lernens klarer. Eine Routine kann sich entwickeln. Die initial hohen Zeitkosten des organisatorischen Aufwandes fallen dann weg. In großen Lerngruppen soll man kleine Veränderungen Schritt für Schritt einführen, sodass die Lernenden den Grund und den Sinn dieser neuen Unterrichtsmethode verstehen (vgl. Schwerdtfeger 2007, S. 255; Becker-Sliwa 2017, S. 48; Loo 2012, 59,100, 2017, S. 7). Die Lernenden brauchen Zeit zu lernen, wie sie mit der Gruppenarbeit umgehen sollen (vgl. Hess 2007, S. 112). 
Das heißt, dass die Einführung einer neuen Methode ein sich wiederholender Prozess sein kann. Nach jedem Einsatz einer neuen Aktivität kann man mögliche Verbesserungen oder Veränderungen vornehmen, bis die Aktivitäten für die Zielgruppe, das Lernziel und die zur Verfügung stehenden Ressourcen geeignet sind. Der Prozess wird durch das folgende Diagramm dargestellt:

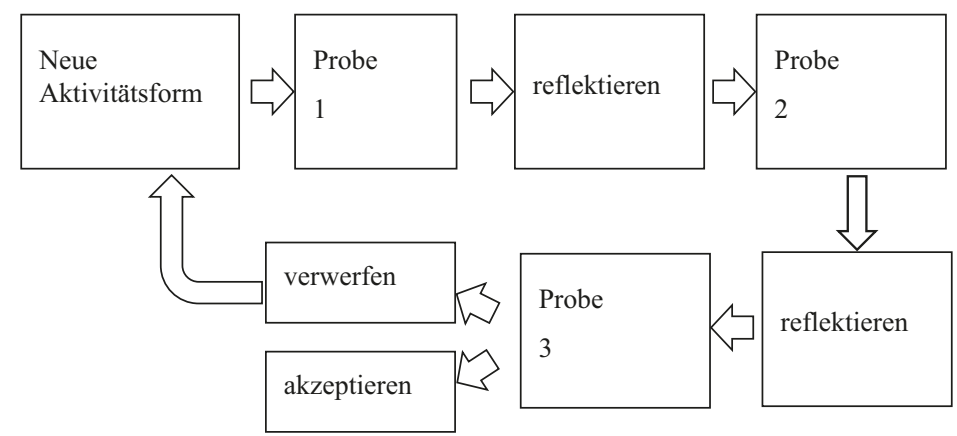

Abbildung 17: Entwicklungsprozess einer neuen Aktivitätsform

Um die Unterrichtszeit effizient zu nutzen und Zeitfresser vorzubeugen, soll der Unterricht in Großgruppen gründlich geplant und vorbereitet werden (vgl. Loo 2017, S. 7). Für die Planung des Unterrichts schlägt Goh (2017, S. 33) die Rückwärtsplanung vor. Das heißt, dass die Auswahl der Aktivitäten von den Lernzielen abhängig ist. Bei der Planung soll die Lehrperson die Zielstellung bedenken, um eine sinnvolle Unterrichtsgestaltung zu entwerfen. Die Aktivitäten sollen Über- und Unterforderung vermeiden (vgl. Loo 2017, S. 5; Rösler 1994, S. 130). Abwechslungen in den Sozial- und Arbeitsformen sollen nicht vergessen werden. Die Lehrperson soll festlegen, wann und wie lange eine Aktivität läuft, um den Unterricht mit klarer Struktur zu gestalten. Außerdem müssen die Raumbedingungen und andere Lehr- und Lernressourcen beachtet werden. Wenn z. B. Gruppenarbeiten in einem Raum mit festgeschraubten Stühlen und Tischen durchgeführt werden müssen, müssen Sitzordnung oder Bewegungsabläufe während der Arbeit gründlich überlegt sein. Ausreichende Materialien zu den Aktivitäten sollten entsprechend der Planung vorbereitet werden.

Die Organisation der Gruppenarbeit spielt dabei die wichtigste Rolle, weil die organisatorischen Dinge und Übergänge von einer Aktivität zu einer anderen in Großgruppen viel Zeit kosten können (vgl. Loo 2017, S. 7). Die Aktivitäten sollen schrittweise durchgeführt werden. Bei der Durchführung von Gruppenarbeit in Großgruppen ist es wichtig, die Aufgaben präzise und eindeutig zu formulieren. 
Die Gruppenbildung hat „entscheidenden Einfluss auf Erfolg oder Misserfolg der Gruppenarbeit“, so Haß (2017b, S. 308). Er schlägt drei Gruppenbildungsverfahren vor: Gruppenbildung durch Entscheidung der Lernenden, durch Entscheidung der Lehrperson oder per Zufall. In der vorliegenden Aktionsforschung wurden meistens Zufallsgruppen gebildet. Haß (ebd.) weist darauf hin, dass der besondere Vorteil dieser Gruppenbildungsmethode ist, den Lernenden das Gefühl zu geben, dass sie im Unterricht fair behandelt werden. Außerdem haben Zufallsgruppen eine Art „Glücksspielfaktor" in sich und sind somit ideal für Sprachlernspiele.

Richtige Gruppierungstechniken können viel Zeit sparen. Im Forschungsexperiment wurden verschiedene Gruppierungsmethoden eingesetzt, die von den Lernenden schnell akzeptiert wurden und zeitlich ökonomisch sind. Manche Gruppierungsmethoden enthalten schon spielerische Elemente wie das Lösen von Rätseln und Glücks- sowie Wettbewerbsfaktoren. Manche Gruppierungsprozesse beinhalten selbst schon eine Aufgabe mit Lernzielen.

Diese Techniken wurden von der Forscherin während der Forschung entwickelt. Da die Probanden mit der Sozialform „Gruppenarbeit“ am Anfang nicht vertraut waren, herrschte immer Chaos, wenn Gruppen gebildet werden sollten. Wenn die Lehrperson sagte:, Wir machen jetzt eine Übung, bitte stehen Sie auf und bilden Sie 5-er Gruppen.", dann konnten die meisten Lernenden mit dieser Anweisung nichts anfangen und wussten nicht, wie oder mit wem sie eine Gruppe bilden könnten. In diesem Fall sind klare und genaue Anweisungen für die Gruppenbildung notwendig. Um den Reibungsverlust zu minimieren, wurden folgenden Gruppierungsmethoden entwickelt:

\section{Gruppierungsmethode A: nach Farben}

Diese Gruppierungstechnik diente hauptsächlich für alle Gruppenaufgaben mit Spielkarteien. Bei der Vorbereitung muss die Lehrperson feststellen, wie viele Gruppen gebildet werden sollen. Je nach Anzahl der Lernenden und der Zahl der zu übenden Redewendungen wird eine passende Gruppengröße bestimmt (wie im Adaptionsbeispiel „Kettenspiel“ im Kapitel 4.2.3). Wenn z. B. fünf Redewendungen geübt werden sollen und es 30 Lernende in der Klasse gibt, sollen 6 Gruppen mit jeweils fünf Mitgliedern gebildet werden. Die Lehrperson schreibt die Redewendungen auf Karten. Jedes Karten-Set für eine Gruppe wird mit einer bestimmten Farbe gekennzeichnet. Im Unterricht verteilt die Lehrperson die Karten vermischt in der Klasse, und sagt $z$. B.: , Alle, die eine rote Karte bekommen haben, bilden bitte hier einen Kreis, diejenigen, die eine blau Karte bekommen haben, bilden dort einen Kreis..."

Mit dieser Methode passiert die Verteilung der Aufgabenmaterialien und das Gruppieren parallel. Die Lernenden wissen dann ganz genau, mit wem, wie und wo sie eine Gruppe bilden. Falls die Raumbedingungen kompliziert sind (wenig freier Raum, nur enge 
Durchgänge zwischen festen Tischen und Stühlen), kann die Lehrperson beispielsweise in einer Powerpoint-Präsentation oder auf der Tafel den Standort jeder Gruppe markieren.

\section{Gruppierungsmethode B: nach Argumenten}

Diese Technik ist ideal für Textarbeit. Die Lehrperson bereitet ein paar Hauptargumente zum Thema des zu bearbeitenden Texts vor, schreibt jedes Argument jeweils auf ein A4 Blatt und verteilt die Blätter in dem Raum. Im Unterricht dient diese Phase als Einführung oder Umstieg von anderen Aktivitäten zum Textverständnis. Um Interesse zu wecken, erklärt die Lehrperson die Argumente zu dem Thema, und bittet die Lernenden, bei dem Argument, dem sie zustimmen (oder das sie ablehnen), aufzustellen.

Die Gruppierung geschieht gleichzeitig mit der Erweckung des Interesses und der Einführung von Redewendungen sowie dem Wortschatz. So bilden die Lernenden mit ähnlichen Meinungen eine Gruppe, und können sich während der folgenden Arbeitsphasen auch gut verstehen und miteinander kommunizieren.

\section{Gruppierungsmethode C: nach Zahlen oder Wortgruppen}

Das ist eine schnelle Gruppierungsmethode, die man mit jeder Gruppenarbeit kombinieren kann. Wenn beispielsweise 40 Studierende anwesend sind und 8 Gruppen gebildet werden sollen, kann die Lehrperson die Studierenden von 1 bis 8 durchzählen lassen. Alle, die 1 gesagt haben, bilden Gruppe 1, die 2 gesagt haben, bilden Gruppe 2, usw. So kann man systematisch in kurzer Zeit Gruppen bilden.

So können auch Anfänger Zahlen auf Deutsch wiederholen und üben. Man kann auch stattdessen bestimmte Wortgruppen nutzen, wie Obst, Kleidung, Farbe usw. Dabei muss man sich nur merken, in welcher Reihenfolge diese Worte genutzt werden. Wenn es in der Klasse viele schwache Lernenden gibt, sollte die Lehrperson die Wörter an die Tafel schreiben, sonst wird dieser Prozess zum Zeitfresser. Vor dem Durchzählen muss auch mehrmals betont werden, dass jeder das gesagte Wort nicht vergessen soll, sonst wissen die Lernenden nachher nicht mehr, zu welcher Gruppe sie gehören.

\section{Gruppierungsmethode D: nach fehlender Information}

Diese Methode ist für Leseverständnisübungen gut geeignet. Der zu lesende Text soll auf einem DIN-A4 Papier nach der Anzahl der Gruppen ausgedruckt oder kopiert werden. Der Text wird in Teile geschnitten, je nach der Anzahl von Mitgliedern jeder Gruppe. Wenn 4 Studierende eine Gruppen bilden sollen, muss der Text in vier Teile geschnitten werden, sodass jeder ein oder zwei Absätze von dem Text bekommt.

Im Unterricht vermischt die Lehrperson alle Zettel und verteilt sie. Die Studierenden sollen mit ihrem Zettel die Gruppenmitglieder finden, mit denen sie den Gesamten Text bilden können und dann entsprechende Aufgaben in der Gruppe machen können.

Für schwache Lernende kann die Lehrperson den zu bearbeitenden Text im Lehrbuch vereinfachen und dann den neuen, leichteren Text mit ähnlichem Inhalt als Gruppenbildungsmaterial benutzen, sodass die Studierenden während der Bildung der Gruppen den schwierigeren Text mit Hilfe der vereinfachten Version spielerisch kennenlernen. Dann verstehen sie später den originalen Text besser und schneller. Diese Gruppierungsmethode kann auch für andere Aufgabetypen adaptiert werden. 
Diese vier Beispiele von Gruppenbildungsmethoden aus der empirischen Forschung zeigen, dass Gruppenbildung nicht nur zeitlich ökonomisch für Großgruppenunterricht, sondern auch ein sinnvoller Lehr- und Lernprozess mit spielerischen Elementen sein kann. Außerdem dient die Gruppierungsphase als ein sanfter Umstieg zwischen Sozialformen und Unterrichtsphasen. Eine gute Gruppierungsmethode kann als eine interaktive/kommunikative spielerische Aufgabe betrachtet werden.

Manche Lehrenden sind der Meinung, dass Sprachlernspiele zeitlich aufwendig seien (vgl. Liang 2018, S. 103). Auch die hospitierenden Lehrenden in der Aktionsforschung haben diese Meinung geteilt (siehe Anhang 20 Lehrerin 6 zu Frage 6). Hier muss man sich zuerst die Frage stellen: Können traditionelle Übungen genau so viele effektive Übungsmöglichkeiten wie Sprachlernspiele anbieten? Ist die zeitliche Sparsamkeit von traditionellen Übungen ein echter Vorteil, oder eher eine oberflächliche Behandlung vom Lernstoff? Während alle Lernenden mit Sprachlernspielen in Gruppen- und Partnerarbeit gleichzeitig üben und sprechen können, können traditionelle Übungen im Plenum nur Sprechmöglichkeit für einzelne, max. zwei Lernende anbieten. Außerdem kann ein hoher Zeitaufwand von Sprachlernspielen oft auf eine mangelhafte Organisation zurückgeführt werden. Das heißt, wenn man ein Sprachlernspiel sorgfältig vorbereitet und organisiert, kann das Spiel auch zeitlich ökonomisch sein.

Basierend auf der Diskussion über Gruppenarbeit im Großgruppenunterricht im Kapitel 2.2.3, dem gesamten Lernfortschritt in zwei Semestern, und der oben angeführten Analyse lässt sich feststellen: obwohl die Lehrenden und auch die Studierenden Zeit brauchten, um sich an die neuen Methoden zu gewöhnen, konnte der Reibungsverlust minimiert werden und die lernerzentrierte spielerische Gruppen- und Partnerarbeit zeitlich genau so effizient wie traditionelle lehrerzentrierte Übungsformen organisiert werden, nachdem die richtigen Methoden etabliert waren.

Außerdem kann man anhand der gleichen Menge an Lernstoff in beiden Gruppen in zwei Semestern feststellen, dass die spielerischen Methoden zeitlich genauso effizient wie traditionelle Methoden sind. Im Folgenden wird versucht, die Einzelheiten des zeitlichen Ablaufs zu veranschaulichen.

Mit Hilfe der Didaktisierung und des Lehrtagebuchs wird die Dauer einzelner Unterrichtsaktivitäten von jeder Sitzung notiert, um festzustellen, ob die spielerischen Aktivitäten zeitlich anders ablaufen als die entsprechenden traditionellen Aufgaben. Die Informationen über die einzelnen Aktivitäten mit Sprachlernspielen werden im Anhang 15 zusammengefasst. 
Im Folgenden soll die Signifikanz des Unterschiedes mit Hilfe des MWUTests für unabhängige Stichproben geklärt werden. Für den MWU-Test lautet die Hypothese:

Es besteht kein Unterschied zwischen den zeitlichen Werten der EG und der KG.

Tabelle 55: Ergebnisse von MWU-Tests über die Aktivitätsdauer WS 2016-17 und SS 2017

\begin{tabular}{cccccccc}
\hline & Mittelwert & $\begin{array}{c}\text { Mann- } \\
\text { Whitney- U }\end{array}$ & $\begin{array}{c}\text { Wilcoxon } \\
\text { W }\end{array}$ & Z & $\begin{array}{c}\text { Asymp. Sig. } \\
\text { (zweiteilig) }\end{array}$ & $\begin{array}{c}\text { P. } \\
\left(=\begin{array}{c}\text { Asymp. } \\
\text { Sig./ 2) }\end{array}\right.\end{array}$ \\
\hline WS & & & & & & & \\
2016-17(39) & KG(39) & & & & & \\
\hline SS 2017 & EG(33) & KG(33) & & & & & \\
\cline { 2 - 8 } & 33.02 & 33.98 & 528.500 & 1089.500 & -.206 & .837 & $0.419>0.05$ \\
\hline
\end{tabular}

Die obere Tabelle zeigt, dass der MWU-Test beim Vergleich der beiden Semester keine statistische Signifikanz erreicht $(\mathrm{p}>0.05)$. Es besteht somit kein signifikanter Unterschied zwischen den beiden Gruppen. Dies bestätigt die Hypothese: Es besteht kein zeitlicher Unterschied zwischen spielerischen Aktivitäten und den originalen Übungsformen.

Somit konnte gezeigt werden, dass Sprachlernspiele zeitlich ökonomisch sein können, solange sie sorgfältig organisiert werden. Im folgenden Kapitel wird die Organisations- und Adaptionsmethode von Sprachlernspielen anhand der gesammelten Forschungsdaten näher betrachtet, um eine effiziente Herangehensweise an die Adaption von verschiedenen Inhalten für Sprachlernspiele zu veranschaulichen.

\subsection{Spieladaption: Kreativität und Adäquatheit}

In der vorliegenden Forschung wird kein Spiel aus Spielesammlungen direkt im Unterricht verwendet: Die eingesetzten Sprachlernspiele sind aus traditionellen Übungen nach Spielmustern adaptiert. Einerseits wird so die Validität der Forschung gesichert, weil die Störvariable kontrolliert wird: Die EG und die KG haben die gleichen Lerninhalte und die gleichen Übungen. Nur die Durchführung der Übungen unterscheiden sich bei den beiden Gruppen. Andererseits ist diese Herangehensweise sehr praxisorientiert, weil Spiele aus Spielesammlungen meistens den konkreten Unterrichtssituationen nicht entsprechen (vgl. Hoppe 
1985, S. 5). Außerdem gibt es auf dem chinesischen Buchmarkt kaum Spielesammlungen für den DaF-Unterricht. Die deutschen Spielesammlungen sind auch für viele Lehrenden in China nur schwer zugänglich. Adaption von Übungen aus vorhandenen Lehrwerken ist für viele Lehrende leichter zu bewältigen.

Koenig (2003, S. 11) betont, dass die Durchführung von Übungen und Spielen im Unterricht „einfacher gesagt als getan“ ist, weil hierbei die Kreativität der Lehrperson gefragt ist. Aus diesen Gründen widmet sich dieses Unterkapitel den Lehrenden in der Praxis und bietet einen Adaptionsleitfaden von traditionellen Übungen als Sprachlernspiele an. Eine Reihe von Adaptionsvorschlägen wird vorgestellt. Die Adäquatheit wird mit Hilfe der Analyse von Lehrtagebuchbeiträgen aus der Forschung überprüft, um die Schwierigkeiten und Möglichkeiten beim Spieleinsatz im DaF-Unterricht in China unter die Lupe zu nehmen.

In der 4. Auflage vom Handbuch Fremdsprachenunterricht (Bausch 2007) wird der Beitrag zum Sprachlernspiel von Kleppin (2007) der Kategorie "Sozialform“ zugeordnet. In der Tat kann ein Sprachlernspiel als Sozialform von Unterrichtsaktivitäten verstanden werden. Wenn eine Übung kreativ durchgeführt wird, wird sie ein Spiel. Fast jede Übung kann zum Spiel adaptiert werden (vgl. Kleppin 2007, S. 265; Koenig 2003, S. 11).

Koenig (2003, S. 11-13) erörtert, dass die traditionellen Übungen häufig die interaktiven sowie kreativspielerischen Perspektiven vom Fremdsprachenunterricht vernachlässigen. Aber wenn die Lehrenden eine Art von kreativer „Redefinitionsfähigkeit“ besitzen, sind sie in der Lage, eine traditionelle Übung ohne viel Aufwand zu variieren. So wird eine traditionelle Übung auch motivierend, lernorientiert, interaktiv und spielerisch.

Klippel (1995, S. 115) schlägt vor, dass in der Lehrfortbildung zum Thema Spieleinsatz erstmal konkrete Spielbeispiele gezeigt und dann Adaptionsmethoden vorgestellt werden sollen, sodass die Lehrenden nicht nur ein einzelnes neues Spiel kennenlernen, sondern auch spielerische Prototypen beherrschen, die sie in der Zukunft nach Bedürfnissen und Unterrichtsrealität variieren können. Das heißt, wenn man die Grundmuster von Sprachlernspielen kennengelernt hat, kann man darauf aufbauend andere Sprachlernspiele selbst entwickeln. Solche Grundmuster findet man z. B. in Spielesammlungen (Friedrich und Jan 1990; Lohfert 1983; MacEdonia 2007; Sánchez Benito et al. 2007) oder der Fernkurseinheit zum Sprachlernspiel vom Goethe Institut (Dauvillier und Lévy-Hillerich 2008). Die Sprachlernspiele im empirischen Teil der vorliegenden Forschung basieren größtenteils auf Spielprototypen aus den oben genannten Werken.

Für den Spieleinsatz ist es allerdings von großer Bedeutung, dass das Spiel lernzielgenau, themen- und zielgruppenadäquat eingesetzt wird (vgl. Kacjan 
2010, S. 1180). Dauvillier und Lévy-Hillerich (2008, S. 46) empfiehlt, dass man beim Einsatz von Sprachlernspielen folgende Aspekte berücksichtigen soll:

1. Sprachlernspiele sollen die Lernenden nicht überfordern;

2. Vor dem Einsatz sollen die Spielmaterialien sorgfältig überprüft werden;

3. Die räumlichen Bedingungen sollen für das Spiel geeignet sein;

4. Der Aufwand des Spiels soll genau überlegt werden.

Es gibt eine Reihe von wichtigen Einsatzkriterien zum Sprachlernspiel, die man bei der Adaption berücksichtigen soll. Im Folgenden werden die wichtigsten Kriterien zur Vorbereitung, Durchführung und Nacharbeitung des Spieleinsatzes veranschaulicht.

Wenn es eine wichtigste Phase des Spieleinsatzes gibt, so ist dies zweifellos die Vorbereitung. Jedes Spiel muss für jede Verwendung entsprechend vorbereitet werden. Dauvillier und Lévy-Hillerich (2008, S. 78) schlägt folgende Checkliste vor:

1. Ist der Spieltyp geeignet?

2. Ist der Spieltyp für die jeweilige Lerngruppe geeignet?

3. In welche Unterrichtsphase passt das Spiel?

4. Wann wird gespielt?

Einer der Nachteile von Sprachlernspielen ist der hohe Zeitaufwand bei der Vorund Nachbereitung und der Materialaufwand (vgl. Mátyás 2010, S. 396), besonders wenn die Lerngruppe groß ist. Bei der Vorbereitung des Spiels soll man nämlich den Aufwand und die Lernziele des Spiels besonders beachten. Kleppin (2007, S. 265) legt darauf viel Wert und betont, dass der Spielaufwand mit den zu erwartenden Effekten in einem sinnvollen Verhältnis stehen soll. Wenn ein Spiel zu aufwendig vorbereitet werden muss, soll die Lehrperson überlegen, ob der Aufwand sich lohnt, und ob das Spiel das Lernen wirklich effizient begünstigen kann.

Man kann z. B. die traditionellen Übungen aus dem Lehrwerk mit Wettbewerbselementen integrieren, ohne zusätzliche Spielmaterialien vorzubereiten. So erreicht man das Lernziel und auch das Spielziel ohne viel Aufwand. Es gibt auch Spiele, für die die Lehrperson Spielkarten aus Übungen des Lehrbuches basteln soll, die anschließend mit anderen Gruppen weiter benutzt werden können (siehe Adaptionsbeispiel 1 im Kapitel 4.2.3).

Außerdem ist bei der Vorbereitungsphase wichtig, das Spiel zielgruppengeeignet zu planen. Das heißt, dass das Spiel nicht unter- oder überfordernd sein soll. Hansen (1990, S. 37) zeigt auf, dass die sprachliche und inhaltliche Vorbereitung für einen reibungslosen Spielverlauf sehr wichtig ist. Die Lernenden sollen die nötigen sprachlichen Wendungen beherrschen. Die Lehrenden sollen 
die nötigen Redemittel bereitstellen, sodass die sprachlichen Hilfsmittel für die Lernenden, insbesondere für schwache Lernende, zur Verfügung stehen. Für die Binnendifferenzierung soll man auch vorher planen (vgl. ebenda, S. 38). Man kann z. B. Zusatzmaterialien oder Zusatzanweisungen für starke Lernende vorbereiten, sodass sie sich nicht langweilen.

Da klare und knappe Spielanweisungen für die Durchführung des Spiels sehr wichtig sind (vgl. Hansen und Wendt 1990, S. 38; Kleppin 2007, S. 265; Behme 1985b, S. 20; Dauvillier und Lévy-Hillerich 2008, S. 81), ist es nötig, dass die Lehrperson bei der Vorbereitung die Anweisungen gut überlegt oder versucht, die Spielanleitung zu visualisieren. Damit kann man viel Zeit im Unterricht sparen, besonders wenn die Lernenden wenig Erfahrung mit Sprachlernspielen haben.

Am Ende der Vorbereitung sollen die Materialien sorgfältig überprüft werden, vor allem, wenn die Lehrperson zusätzliche Materialien basteln muss. Für Kartenspiele wie „Memory“ oder „Domino“ muss man sicherstellen, dass kein Kärtchen fehlt. Die Zusammengehörigkeit der Karten soll auch überprüft werden. Die Vollständigkeit der Spielmaterialien spielt eine entscheidende Rolle für eine reibungslose Durchführung (vgl. ebenda, S. 78-80).

Die räumlichen Bedingungen sind ein anderer wichtiger Faktor, den man bei der Auswahl und Vorbereitung des Spiels berücksichtigen muss. Außer den technischen Voraussetzungen betont Behme (1985b, S. 20), dass das Sitzen in Kreisform sowie lärmgeschützte Räumlichkeiten für kommunikative spielerische Übungen wichtig sind.

Die Raumbedingung ist dabei nicht immer ideal. In China sind z. B. in Klassenräumen meistens festgeschraubte Tische und Stühle zu finden (vgl. Becker-Sliwa 2017, S. 47). Nach Erfahrung der Forscherin können ungünstige Raumbedingungen allerdings mit passenden Tricks umgangen werden. Aktivitäten, die viel Zusammenarbeit mit Karten haben und bei denen viel geschrieben werden muss, wie beispielsweise Domino, Satzbaukastenspiel sowie Kreuzworträtsel, können als Partnerarbeit mit dem Sitzpartner geplant werden. Partnerarbeit ist für solche Spieltypen besser geeignet, weil die Lernenden bequem sitzen und zusammenarbeiten können, statt sich ständig umzudrehen. Dabei muss genügend Material zur Verfügung stehen, so dass die Lernenden gut damit arbeiten können. Interaktive, kommunikative Gesprächsspiele mit größeren Gruppen (ca. 5 bis 7 Teilnehmer) können im Stehen im Kreis organisiert werden, dann stören festgeschraubte Tische und Stühle nicht mehr. Die Lernenden können eine beliebige Stelle im Klassenraum für die eigene Gruppe auswählen. Außerdem kann die Bewegung gegen Müdigkeit helfen. 
Wenn man sich bei der Vorbereitung genügend Gedanken gemacht hat, sollte die Durchführung eines Spiels relativ reibungslos ablaufen, wenn die vorbereiteten Materialien im Unterricht zur Verfügung stehen. Die Forscherin hat z. B. während des Experiments zwei Mal die Materialien zu Hause auf dem Tisch liegen lassen ${ }^{30}$.

Bei der Durchführung spielen die Regelformulierung und Präsentation der Sprachlernspiele eine entscheidende Rolle. Die Lernenden müssen die Spielzeit und - regeln gut verstehen. Bei der Gruppenarbeit ist die Zusammensetzung der Gruppe auch sehr wichtig (siehe Kapitel 6.1). Zur Durchführung und Organisation der Sprachlernspiele bietet Schweckendiek (2001, S. 19) ein paar ausführliche Anweisungen und Kriterien an, die in der Praxis helfen können.

In der Praxis tauchen allerdings immer wieder unerwartete Situationen während der Durchführung auf. Wenn man Kartenspiele für 40 Personen vorbereitet hat, aber nur 30 Lernende anwesend sind, muss man eine andere Gruppierungsstrategie benutzen. Es ist empfehlenswert, während der Vorbereitungsphase mehr Materialien als nötig vorzubereiten.

Die Rolle der Lehrperson spielt bei der Durchführung ebenso eine bedeutsame Rolle. Auf keinen Fall sollen die Interaktion und die sprachliche Produktion der Lernenden während des Spiels von der Lehrperson bewertet oder benotet werden. Sprachlernspiele sind immer sanktionsfrei (vgl. Kleppin 2007, S. 264; Behme 1985b, S. 20; Dauvillier und Lévy-Hillerich 2008, S. 40). Das spielende Lernen soll angstfrei gestaltet sein. Dauvillier und Lévy-Hillerich (2008, S. 41) schlägt folgenden Grundsatz vor:

1. Die Lehrperson soll das Spielgeschehen nicht stören.

2. Nach dem Spiel darf, kann, soll über Fehler gesprochen werden.

3. Nicht alle Fehler müssen verbessert werden.

Die Lehrperson kann mitspielen, beispielsweise als Moderatorin beim Lottospiel oder Auktionatorin beim Auktionsspiel. Die Lehrperson kann auch als Helfer für sprachliche Richtigkeit dienen (vgl. Kleppin 2007, S. 266; Hansen und Wendt 1990, S. 39), sowie thematische und organisatorische Hinweise geben. Oder die Lehrperson kann den Prozess beobachten, um die Fähigkeit der Lernenden zu evaluieren (vgl. Hansen und Wendt 1990, S. 39).

Nach der Durchführung gibt es eine Nachbearbeitungsphase. Die Lehrperson reflektiert das Spiel und dessen Umsetzung und stellt sich Fragen zur

30 Die peinliche Situation wird vermieden, wenn man mehr Erfahrungen mit den Sprachlernspielen gesammelt hat. So kann man vor Ort ohne Zusatzmaterialien Übungen ins Spiel zaubern. Dann hat man immer einen Back-up Plan im Kopf. 
Organisation. Die Reflexion hilft der Lehrperson, die Spiele zu verbessern. Gute, erfolgreiche Spiele können archiviert werden. Zur Spielarchivierung bietet Dauvillier und Lévy-Hillerich (2008, S. 85-87) ausführliche Hinweise an.

Zusammenfassend lässt sich sagen, wenn man ein Spiel im Voraus sehr gewissenhaft vorbereitet hat, steht einer gelungenen Durchführung nichts im Wege. Aber auch für erfahrene Lehrende kann ein Spiel mal nicht so gut wie geplant laufen. Solange man jedoch bereit ist, aus Fehlern zu lernen, wird es gelingen (vgl. ebenda, S. 78).

Im Lehrtagebuch aus der vorliegenden Forschung gibt es im WS 2016 insgesamt 16 Textsegmente zu Schwierigkeiten in der Organisation von Sprachlernspielen; im SS 2017-18 hingegen nur 4 (siehe Tabelle 48 und Tabelle 49). Das heißt, dass eine erfahrene und sich reflektierende Lehrperson ihre organisatorischen Fähigkeiten bezüglich der Sprachlernspiele stets verbessern kann. Die organisatorischen Probleme werden im Anhang 17 tabellarisch zusammengefasst. Hier findet man einen vereinfachten Überblick über die kategorisierten Probleme, um die Schwierigkeiten des Spieleinsatzes zu veranschaulichen:

Tabelle 56: Textsegmentenanzahl zu den ungelungenen organisatorischen Problemen

\begin{tabular}{|c|c|}
\hline Grund der ungelungenen Organisation & Anzahl der Textsegmente \\
\hline \multicolumn{2}{|c|}{ WS 2016-17 } \\
\hline Mangel an Zeit & 2 \\
\hline Unklare Anweisung & 1 \\
\hline Erfahrungsmangel (lernerseits) & 2 \\
\hline Fehlende Materialien & 6 \\
\hline Überforderung & 4 \\
\hline Große Anzahl der Lernenden & 1 \\
\hline \multicolumn{2}{|l|}{ 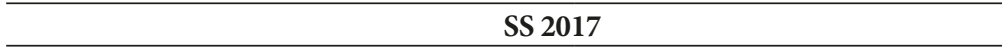 } \\
\hline Überforderung & 2 \\
\hline Mangel an Zeit & 1 \\
\hline Sonstige Probleme lernerseits & 2 \\
\hline
\end{tabular}

Aus der Tabelle wird ersichtlich, dass die meisten organisatorischen Probleme im WS 2016-17 wegen mangelnder Vorbereitung verursacht werden. Fehlende Materialien spielen dabei die größte Rolle. Danach folgt die Überforderung der Lernenden d. h. zielgruppenungeeignete Spieleinsätze. Im SS 2017 gab es keine Probleme mit Materialien. Die bei den Lernenden beobachteten Probleme hatten nichts mit Sprachwissen zu tun, sondern bezogen sich auf die allgemeinen Fähigkeiten zum Kontextverständnis oder fehlende Motivation. 
Es zeigt sich, dass die Vorbereitung für die Durchführung des Spiels bedeutsam ist. Die Lehrperson braucht auch Zeit, um Erfahrung mit Sprachlernspielen zu sammeln und die Zielgruppe kennen zu lernen, sodass sie das Spiel richtig vorbereiten kann. Infolgedessen werden die wichtigen Reflexionen aus dem Lehrtagebuch zu den organisatorischen Methoden aus den zwei Semestern dargestellt, um die Einsatzprobleme und deren Lösung zu kristallisieren.

Tabelle 57 und Tabelle 58 zeigen, dass die Vorbereitung beachtet werden muss $(1,2)$ und dass Gruppierungsmethoden bei der Durchführung eine wichtige Rolle spielen können $(4,7,8,9)$. Die Reflexionen 11, 12 und 16 veranschaulichen auch, dass die räumlichen Bedingungen den Spieleinsatz beeinflussen können. Man kann aber die Spieltypen dem Raum anpassen. Das spricht wieder für die Wichtigkeit der Vorbereitung. Die Reflexionen zum zeitlichen Aspekt (3, 4, 13) zeigen, dass, obwohl das Spiel mehr Zeit kostet, man mit den richtigen Methoden den Reibungsverlust verringern kann. Außerdem lohnt sich der Zeiteinsatz, weil man durch das Spielen mehr Übungsmöglichkeiten anbieten kann.

Betrachtet man die Analyse des Tagebuchs, können folgende Faktoren festgesellt werden: Die Lernprozessbewertung und Reflexionen zeigen, dass die Lehrperson im ersten Semester mehr reflektiert hat und bei der Unterrichtsorganisation mehr Problemen begegnet ist. Im zweiten Semester gibt es deutlich weniger ungelungene Organisation als im ersten Semester.

Zusammenfassend lässt sich sagen, wenn die Lehrperson in der Lage ist, aus ihren Fehlern zu lernen und reflektiert damit umzugehen, dann können interaktive/kommunikative Sprachlernspiele im chinesischen DaF-Unterricht eingesetzt werden. Die häufig genannten Stolpersteine (wie große Lerneranzahl und räumliche Bedingungen wie festgeschraubte Tische und Stühle) sind eher Hindernisse, die man mit Kreativität und den richtigen Strategien überwinden kann.

Auch die statistischen Ergebnisse der Items 2, 12 und 13 von Beobachtungsbogen B der Unterrichtsbeobachtung bestätigen, dass die Sprachlernspiele nicht zeitaufwendiger im Unterricht sind und für die Räumlichkeiten genauso geeignet sind wie traditionelle Methoden (siehe Anhang 12). 
Tabelle 57: Wichtige Reflexionen aus dem Lehrtagebuch zur organisatorischen Methode WS 2016-17

\begin{tabular}{|c|c|c|c|c|}
\hline \multirow[t]{2}{*}{$\begin{array}{l}\text { Zur Vor- } \\
\text { bereitung }\end{array}$} & & 1 & $\begin{array}{l}\text { Das Spiel soll sehr sorgfältig vorbereitet werden, } \\
\text { um den reibungslosen Ablauf zu sichern. }\end{array}$ & 11.10 .2016 \\
\hline & & 2 & $\begin{array}{l}\text { Die Vorbereitung fürs Spiel ist unzureichend. Das } \\
\text { führt dazu, dass nicht alle Übungsziele erreicht } \\
\text { werden können. }\end{array}$ & 17.10 .2016 \\
\hline \multirow[t]{10}{*}{$\begin{array}{l}\text { Zur } \\
\text { Durchfüh- } \\
\text { rung }\end{array}$} & Zeitlich & 3 & $\begin{array}{l}\text { Zeit bei der EG ist etwas knapp, bei der KG } \\
\text { ausreichend. Das scheint aber mit dem Spiel } \\
\text { kaum zusammenzuhängen, eher mit den } \\
\text { Lerngewohnheiten von Studierenden. }\end{array}$ & 10.10 .2016 \\
\hline & & 4 & $\begin{array}{l}\text { Die Gruppierung nimmt immer noch viel Zeit in } \\
\text { Anspruch, ist aber schon besser als am Anfang. }\end{array}$ & 24.10 .2016 \\
\hline & $\begin{array}{l}\text { Organisa- } \\
\text { torisch }\end{array}$ & 5 & $\begin{array}{l}\text { Das (interaktive) Spiel soll mit schriftlicher Übung } \\
\text { gut integriert sein, sodass es genügend Zeit für } \\
\text { die Beruhigung nach der Aufregung gibt. Sonst } \\
\text { können die Studierenden nach dem Spiel nicht } \\
\text { konzentriert arbeiten. }\end{array}$ & 11.10 .2016 \\
\hline & & 6 & $\begin{array}{l}\text { Man muss die Einsatzphase des Spiels gut } \\
\text { überlegen, Spiele mit viel Interaktion sollen eher } \\
\text { am Ende der Stunde eingesetzt werden. }\end{array}$ & 11.10 .2016 \\
\hline & & 7 & Gruppierungsmethode beachten!!! & 24.10 .2016 \\
\hline & & 8 & $\begin{array}{l}\text { Das erste Mal Gruppierung mit Hilfe von mit } \\
\text { Farben gekennzeichneten Spielkarten. Hat sehr } \\
\text { gut funktioniert. Man sieht auch ganz klar, ob jede } \\
\text { Person zu der richtigen Gruppe gegangen ist (aber } \\
\text { nicht einfach da sitzt wo sie will). Gruppierung } \\
\text { läuft auch schneller. }\end{array}$ & 7.11.2016 \\
\hline & & 9 & Gruppierung hat Zeit gekostet. & 8.11 .2016 \\
\hline & $\begin{array}{l}\text { Menge der } \\
\text { Lernenden }\end{array}$ & 10 & $\begin{array}{l}\text { Wegen der großen Anzahl von Lernenden ist } \\
\text { es schwer zu kontrollieren, ob jeder Lernende } \\
\text { Deutsch gesprochen hat. }\end{array}$ & 8.11 .2016 \\
\hline & $\begin{array}{l}\text { Räumlich- } \\
\text { keit }\end{array}$ & 11 & $\begin{array}{l}\text { Wegen räumlicher Bedingungen des } \\
\text { Klassenzimmers können die Studierenden nicht } \\
\text { sehr gut miteinander arbeiten. }\end{array}$ & 22.11.2016 \\
\hline & & 12 & $\begin{array}{l}\text { Der Klassenraum von dem Bowen Gebäude ist für } \\
\text { Gruppenarbeit ungeeignet. }\end{array}$ & 20.12 .2016 \\
\hline
\end{tabular}


Tabelle 58: Wichtige Reflexionen aus dem Lehrtagebuch zur organisatorischen Methode SS 2017

\begin{tabular}{|c|c|c|c|}
\hline \multirow[t]{4}{*}{$\begin{array}{l}\text { Zur } \\
\text { Durchfüh- } \\
\text { rung }\end{array}$} & Zeitlich & $\begin{array}{l}13 \text { Das Spiel kostet mehr Zeit. Aber bei der } \\
\text { traditionellen Methode muss die Lehrperson } \\
\text { die Antwort geben und jede Aufgabe im } \\
\text { Plenum korrigieren. Beim Spiel können } \\
\text { die Studierenden die Antwort mit Hilfe } \\
\text { der Struktur des Spielmaterials selbst } \\
\text { zusammenfassen. }\end{array}$ & 01.04 .2017 \\
\hline & Organisatorisch & $\begin{array}{l}14 \text { Die Mehrheit der Lernenden kann beim Spiel } \\
\text { aktiv teilnehmen, einige nicht. Es ist schwer } \\
\text { zu beobachten, wer wirklich teilgenommen } \\
\text { hat. }\end{array}$ & 14.04.2017 \\
\hline & & $\begin{array}{l}15 \text { Zum ersten Mal lässt die Lehrperson die } \\
\text { Studierenden das Spiel selbst basteln. Es war } \\
\text { nicht kompliziert, alle haben Interesse gezeigt. }\end{array}$ & 17.04.2017 \\
\hline & räumlich & $\begin{array}{l}16 \text { Es gibt einige Lernende, die faulenzen und } \\
\text { die Übung vom Partner machen lassen. } \\
\text { Wahrscheinlich teils wegen festgeschraubter } \\
\text { Tische und Stühle. }\end{array}$ & 13.03.2017 \\
\hline
\end{tabular}

\subsection{Kriterien für bevorzugte Spieltypen}

Die oben genannte Herangehensweise von Adaption und Durchführung der Sprachlernspiele veranschaulicht, dass Sprachlernspiele für das Interesse und die Vorlieben der Zielgruppe und deren Lernziele adäquat eingesetzt werden können. Es stellt sich die Frage: Gibt es einen von chinesischen Lernenden bevorzugten Spieletyp? Um diese Frage beantworten zu können, muss man die Ergebnisse der Befragungen über den Spieleinsatz in der Aktionsforschung analysieren. Die Analyse erfolgt mit Hilfe des „Kruskal-Wallis H“ Test durch SPSS. Der „Kruskal-Wallis $\mathrm{H}^{\text {“ }}$ Test dient der Überprüfung von signifikanten Unterschieden für Daten, die aus mehr als drei Gruppen bestehen (vgl. Qin und Bi 2016, S. 340).

In der empirischen Forschung werden zu jedem eingesetzten Spiel folgende sechs Items zur Bewertung gestellt: 
Tabelle 59: Beispiel der Items zum eingesetzten Spielen

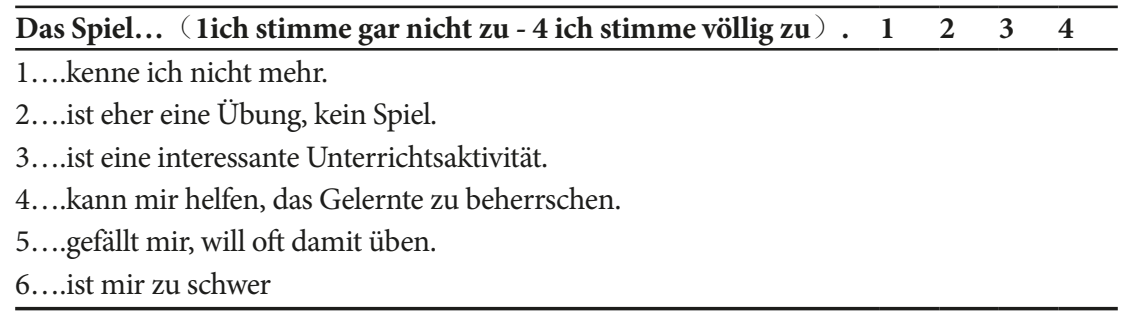

Jedes Spiel wird von Studierenden mit den oben aufgeführten 6 Items bewertet, im WS 2016-17 wurden 21 Fragenbögen in die Auswertung einbezogen, im SS 2017 waren es 25. Mit Hilfe einer deskriptiven Analyse durch SPSS wurden die Mittelwerte der jeweiligen Items berechnet (siehe Anhang18). Danach wurden die Mittelwerte von jedem einzelnen Item zu jeder Frage von allen eingesetzten

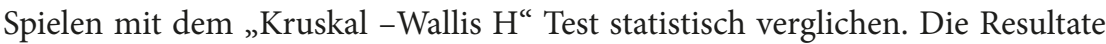

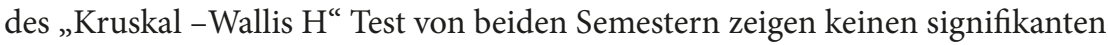
Unterschied:

Tabelle 60: Ergebnisse von „Kruskal -Wallis H“ Tests WS 2016-17

\begin{tabular}{lcccc}
\hline Item & Mittelwert & Chi-Square & df & $\begin{array}{c}\text { Asymp. Sig. } \\
(\text { zweiteilig })\end{array}$ \\
\hline 1 & 1.5450 & 8.000 & 8 & $.433>0.05$ \\
2 & 2.7566 & 8.000 & 8 & $.433>0.05$ \\
3 & 3.1746 & 8.000 & 8 & $.433>0.05$ \\
4 & 3.0265 & 8.000 & 8 & $.433>0.05$ \\
5 & 3.0318 & 8.000 & 8 & $.433>0.05$ \\
6 & 2.2011 & 8.000 & 8 & $.433>0.05$ \\
\hline
\end{tabular}

Tabelle 61: Ergebnisse von „Kruskal -Wallis H“ Tests SS 2017

\begin{tabular}{lcccc}
\hline Item & Mittelwert & Chi-Square & df & $\begin{array}{c}\text { Asymp. Sig. } \\
\text { zweiteilig })\end{array}$ \\
\hline 1 & 1.2914 & 6.000 & 6 & $.423>0.05$ \\
2 & 2.6914 & 6.000 & 6 & $.423>0.05$ \\
3 & 3.2571 & 6.000 & 6 & $.423>0.05$ \\
4 & 3.1886 & 6.000 & 6 & $.423>0.05$ \\
5 & 3.1829 & 6.000 & 6 & $.423>0.05$ \\
6 & 2.0000 & 6.000 & 6 & $.423>0.05$ \\
\hline
\end{tabular}


Die statistischen Resultate zeigen, dass kein Spiel hinsichtlich der 6 Kriterien anders von den Studierenden bewertet wird. Die Studierenden können sich an alle Spiele (Item 1) erinnern, und nahmen die Aktivität als eine Mischung von Übung und Spiel wahr (Item 2). Sie finden die eingesetzten Spiele interessant (Item 3), lernbegünstigend (Item 4) und mögen die Aktivitäten (Item 5). Sie sind auch in der Lage, mit den eingesetzten Spielen zu arbeiten und fühlen keine Überforderung (Item 6).

Mit Hilfe der Befragung kann man auch bezüglich der Sozialformen von Sprachlernspielen eine Präferenz feststellen. In diesem Fragenbogen müssen die Studierenden ihren bevorzugten Spieltyp hinsichtlich Sozialformen auswählen. In beiden Semestern sind Gruppenspiele mit 3-10 Spielern am beliebtesten. Danach folgen Partnerspiel und Einzelspiel.

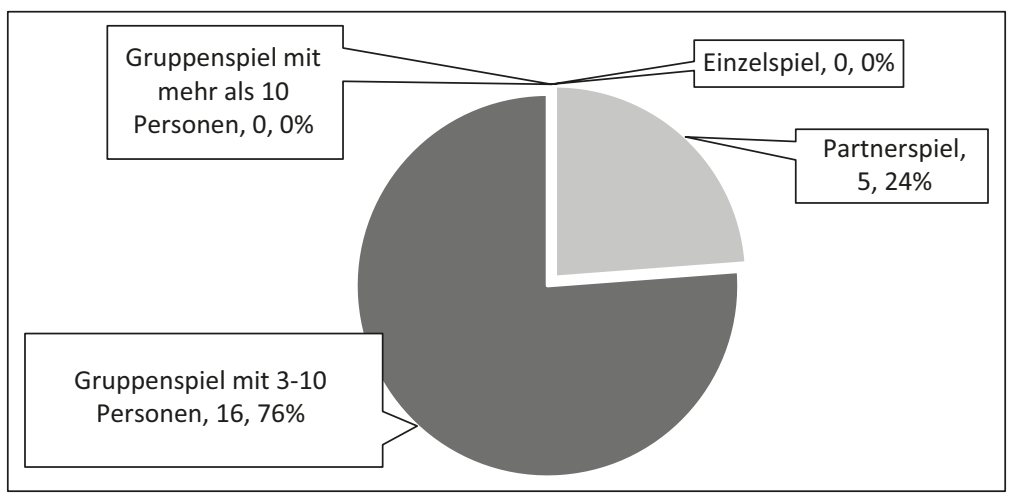

Abbildung 18: Antworten zum bevorzugten Spieltyp WS 2016-17 


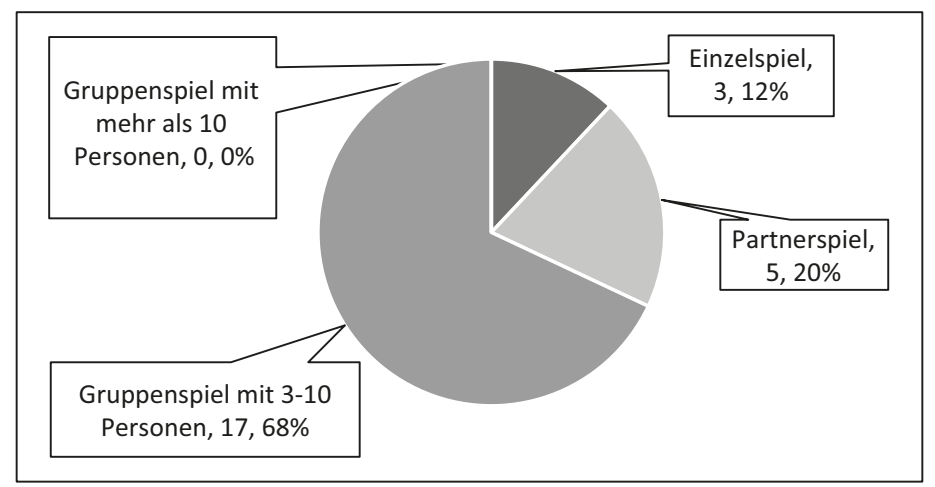

Abbildung 19: Antworten zum bevorzugten Spieltyp SS 2017

Die Studierenden haben viele Gründe genannt (siehe Anhang 19). Im Folgenden wird ein Überblick zur Anzahl der Gründe in verschiedenen Kategorien gegeben:

Tabelle 62: Zusammenfassung der Begründungen zur Sozialform der Sprachlernspiele

\begin{tabular}{llc}
\hline Sozialform & Begründung des Spieleinsatzes & $\begin{array}{c}\text { Anzahl der } \\
\text { Begründungen }\end{array}$ \\
\hline Partnerspiel & Mehr Kommunikationsmöglichkeit & 3 \\
(WS und SS) & $\begin{array}{l}\text { Passende Personenzahl für Interaktion, nicht } \\
\text { zu viel }\end{array}$ & 4 \\
& Zeitlich ökonomisch & 1 \\
Gruppenspiel mit 3-10 & Mehr Möglichkeiten für das Lösen von & 7 \\
Personen & Aufgaben & 20 \\
(WS und SS) & Mehr Kommunikations- und & \\
& Interaktionsmöglichkeiten & 1 \\
& Gutes Lernklima & 1 \\
& Mehr Gesellschaftsmöglichkeiten & 2 \\
\hline
\end{tabular}


Betrachtet man die Gründe kann man folgende Schlussfolgerung ziehen:

Erstens legt die Mehrheit der Lernenden großen Wert auf Kommunikation. Viele wählen Partner- und Gruppenarbeit aus, weil sie der Meinung sind, dass solche Sozialformen für Kommunikation und Interaktion gut geeignet sind. Zweitens kann man sagen, dass die Lernenden die Sprachlernspiele mit Kooperationselementen bevorzugen. Drittens lässt sich vermuten, dass sie in Gruppenspielen Unterstützung von den anderen Lernenden bekommen können und sich wohler fühlen. Sie fühlen sich stärker, wenn sie in der Gruppe arbeiten. Deswegen sind Spiele in Gruppen die bevorzugte Sozialform bei den meisten Befragten.

Auf der anderen Seite wissen viele Studierende auch, dass Partnerarbeit weniger zeitaufwendig ist. Sie schätzen Partnerspiele als eine Art von Sozialform, in der Kommunikation und selbständige Arbeit gut kombiniert werden können. In Klassenräumen, die für Gruppenarbeit ungeeignet sind, kann man trotzdem Partnerspiele einsetzten.

Im Sommersemester 2017 haben drei Studierende geäußert, dass sie lieber alleine arbeiten möchten, 2 davon begründen, dass sie in dieser Sozialform Zeit für selbstständiges Reflektieren haben. Das weist vermutlich darauf hin, dass die Studierenden viel Erfahrung mit Spielen gesammelt und im Unterricht mehr Selbstvertrauen gewonnen haben.

Aktivitäten, die Möglichkeiten für den Austausch von Ideen und Gedanken der Lernenden anbieten können, können die Klasse in eine Gemeinschaft verwandeln, in der die Lernenden sich wohl fühlen und sich äußern wollen. Solche Aktivitäten können ein gutes Lernklima schaffen und die Lernenden zum Lernen motivieren (vgl. Hess 2007, S. 34). Es zeigt sich, dass die chinesischen Lernenden Wert auf Kommunikation, Interaktion und Zusammenarbeit mit anderen legen, solange die Durchführung und Organisation der Sprachlernspiele ihre Vorlieben und Gewohnheiten berücksichtigen. In einer angstfreien Lernumgebung sind sie auch in der Lage, im Unterricht aktiv und kommunikationsfreudig zu sein.

\subsection{Zwischenfazit}

Jetzt können die drei Forschungsfragen aus diesem Kapitel beantwortet werden:

1. Kann der Unterricht mit Sprachlernspielen zeitlich genau so effizient sein wie Unterricht ohne Sprachlernspiele?

Ja. Bei sorgfältiger Vorbereitung und gut geplanter Durchführung können Sprachlernspiele zeitlich genauso effizient wie traditionelle Übungen sein. 
Hinsichtlich Übungsmöglichkeiten haben Sprachlernspiele noch mehr Vorteile als traditionelle Übungen: Einerseits können Sprachlernspiele verschiedene Übungsziele (schriftlich und mündlich) kombinieren. Andererseits können mehrere Lernende beim Sprachlernspiel gleichzeitig üben.

2. Kann man traditionelle Übungsformen als Sprachlernspiele adaptieren?

Ja. Dazu ist es zunächst wichtig, generelle Grundmuster von Sprachlernspielen kennen zu lernen, und darauf aufbauend die Inhalte aus traditionellen Übungen zum Spracherwerb offen und kreativ anzupassen.

3. Kann man Sprachlernspiele in einer größeren Gruppe einsetzen, auch wenn die Rahmenbedingungen nicht ideal für den Spieleinsatz sind?

Ja, man kann. Die vorliegende Forschung zeigt, dass begrenzter Raum sowie große Klassen den Einsatz von Sprachlernspielen beeinflussen können, aber kein unlösbares Problem darstellen. Wichtig ist, dass die Lehrenden sich mit den räumlichen Bedingungen und der Zielgruppe gut auskennen und die Materialien sowie die Durchführungsplanung entsprechend anpassen. Eine große Gruppe kann sogar Vorteile haben: Es bieten sich dann mehr Kommunikationsund Interaktionsmöglichkeiten zwischen den Lernenden untereinander an. Die Großgruppe macht es möglich, abwechslungsreiche Gruppierungen zu organisieren, um den Lernenden verschiedene Kommunikations- sowie Arbeitsgelegenheiten zu verschaffen. Eine Klasse mit 20 bis 40 Personen ist ideal für den Einsatz von Sprachlernspielen und abwechslungsreicher Unterrichtsgestaltung. Kleinere Gruppen (weniger als 20 Personen) bieten nur begrenzte Interaktionsvarianten bzw. Gruppenbildungsmöglichkeiten an. In sehr großen Gruppen (> 40 Personen) wird der Vorbereitungs- und Nachbearbeitungsaufwand schnell sehr hoch. Prinzipiell kann man aber sagen, dass für jede Gruppenstärke geeignete Sprachlernspiele entwickelt werden können.

Es zeigt sich, dass die erfolgreiche Durchführung von Sprachlernspielen möglich, aber nicht unbedingt leicht ist. Die Lehrperson muss in der Lage sein, mit den Materialien kreativ umzugehen. Nicht nur die Lernenden brauchen Zeit, auch die Lehrenden müssen lernen, wie man mit Sprachlernspielen umgehen soll, um diese sinnvoll im Unterricht einsetzen zu können.

Die Balance zwischen dem Aufwand der Organisation und der Wirkung der Sprachlernspiele darf nicht aus den Augen verloren werden. Sprachlernspiele sollen nicht als „Lückenfüller" eingesetzt werden, sondern müssen als eigenständige Übungen mit klar definierten Lernzielen behandelt werden. Mit der richtigen Gruppierungsmethode und motivationsfördernden Wettbewerbs- sowie Glückselementen können traditionelle Übungen ohne aufwändige 
Materialienvorbereitung auch als Sprachlernspiele adaptiert werden. Bis diese jedoch im Unterricht reibungslos und gewinnbringend angewandt werden können, ist ein gewisser Aufwand nötig. Erfahrung und Reflexion helfen den Lehrkräften, bei der Durchführung von Sprachlernspielen immer besser und sicherer zu werden, was sich auf den Lernerfolg der Lernenden auswirkt. 


\section{Fazit und Ausblick}

Die vorangegangene Diskussion hat den Versuch untergenommen, die Wirkung und Akzeptanz von Sprachlernspielen im chinesischen DaF-Kontext zu überprüfen. Die vorliegende Arbeit wurde mit der Hypothese begonnen, dass Sprachlernspiele die Motivation und Leistung der DaF-Lernenden im genannten Kontext fördern und im chinesischen DaF-Unterricht durchaus einsetzbar sein können, solange diese Methode entsprechend adaptiert und sorgfältig verwendet wird. Diese Hypothese wurde mit Hilfe der Aktionsforschung überprüft und bestätigt.

Auf der Motivationsebene hat sich ergeben, dass die Lernenden durch Sprachlernspiele mehr Selbstvertrauen beim Sprachlernen gewonnen haben, sodass sie sich im Unterricht mehr äußern und aktiver teilnehmen. Erhöhtes Selbstvertrauen bezüglich des Fremdsprachenlernens und eine Änderung bei der Einstellung gegenüber der Zielsprache durch den Einsatz von Sprachlernspielen wird auch bestätigt.

Auf der Leistungsebene haben sich im Rahmen der Forschung interessante Ergebnisse herauskristallisiert. Die Implikationen sind vielfältig:

Erstens kann man feststellen, dass Sprachlernspiele das Erlernen von fundamentalem sprachlichem Wissen fördern können. Im Gegensatz zur Kritik mancher chinesischer Lehrenden, dass interaktive/kommunikative Methoden das Erlernen von fundamentalem sprachlichem Wissen nicht fördern können (vgl. Wang 2007, S. 93), zeigt die vorliegende Forschung, dass interaktive/kommunikative Methoden wie Sprachlernspiele das Erlernen von Grammatik- und Wortschatzwissen genauso gut fördern wie traditioneller lehrerzentrierter Unterricht. Darüber hinaus können Sprachlernspiele die kommunikativen Kompetenzen der Lernenden besser als lehrerzentrierter Unterricht ohne Sprachlernspiele fördern.

Zweitens wird die Bereitschaft zur Kommunikation und Interaktion der Lernenden durch Sprachlernspiele deutlich verbessert. Die vermehrten Sprachmeldungen, Aktivitäten und die erhöhte Motivation mittel- und langfristig führen $\mathrm{zu}$ besseren und nachhaltigeren Lernfortschritten. Dabei hat sich gezeigt, dass die Lernenden mit Sprachlernspielen im zweiten Jahr bessere Leistungen zeigen als jene ohne Sprachlernspiele.

Letztendlich soll die Effizienz von Sprachlernspielen in Anbetracht des Aufwands beim Einsatz von Sprachlernspielen erwogen werden. Wenn eine hoch motivierte Gruppe bspw. in kurzer Zeit umfangreiches Wissen für die 
Zielsprache beherrschen soll, sind Sprachlernspiele keine passende Methode. Für schwache Lernende, die keine oder wenig intrinsische Motivation für die Zielsprache besitzen und sich lange Zeit mit der Sprache beschäftigen sollen bzw. müssen, können Sprachlernspiele eine ideale Methode sein, um das Interesse an der zu erlernenden Sprache und die Interaktion im Unterricht zu fördern.

Die vorliegende Forschung bestätigt auch, dass Sprachlernspiele durchaus an chinesische Rahmenbedingungen angepasst werden können und dann auch von chinesischen Lernenden akzeptiert werden. Allerdings brauchen sowohl die Lehrenden als auch die Lernenden Zeit, sich an die neue Methode zu gewöhnen. Da der Einsatz von Sprachlernspielen ein angstfreies und kommunikationsförderndes Lernklima herbeiführen kann, lohnt es sich, die Methode einzusetzen, solange die Methode für die Situation der Zielgruppe geeignet ist. Darüber hinaus können traditionelle Übungen ohne viel Aufwand als Sprachlernspiele adaptiert werden. Das ist für chinesische Lehrende, die mit einem bestimmten Lehrwerk arbeiten (müssen), von großer Bedeutung.

Interessanterweise hat die Befragung von Studierenden ergeben, dass auch die „schweigsamen“ chinesischen Lernenden Wert auf Kommunikation und Interaktion im Fremdsprachenunterricht legen. Sie nehmen Kommunikation und Interaktion als wichtige Unterrichtselemente wahr und beteiligen sich aktiv, wenn die Unterrichtsatmosphäre kommunikationsfördernd ist.

Zusammenfassend lässt sich sagen, dass Sprachlernspiele eine Reihe von Vorteilen haben und für jede Unterrichtsphase und viele Lernziele eingesetzt werden können. Sprachlernspiele sind keine "Motivierungstechnik“ (Kleppin 2017, S. 327), und sollen je nach Zielgruppe, Lernziel und Rahmenbedingungen bewusst eingesetzt werden.

Die vorliegende Forschung zeigt allerdings auch, dass der Einsatz von Sprachlernspielen nicht zu verlängerten Lernzeiten außerhalb des Unterrichtes führt. Die Daten der Befragung zur Motivation (vgl. Item 4) während zweier Semester zeigen keinen signifikanten statistischen Unterschied für Lernzeiten außerhalb des Unterrichts zwischen den beiden Gruppen (siehe Tabelle 63). Die Mehrheit der Studierenden beider Gruppen beschäftigt sich weniger als 3 Stunden pro Woche außerhalb des Deutschunterrichts mit der Zielsprache, viele von ihnen sogar weniger als 1 Stunde. 
Tabelle 63: Übersicht der Lernzeit außerhalb des Unterrichts

\begin{tabular}{lcccc}
\hline $\begin{array}{l}\text { Zeit für Deutschlernen außerhalb des } \\
\text { Unterrichts wöchentlich }\end{array}$ & \multicolumn{2}{c}{ WS 2016-17 } & \multicolumn{2}{c}{ SS 2017 } \\
\hline & KG & EG & KG & EG \\
& $\mathbf{( 3 0 )}$ & $\mathbf{( 2 6 )}$ & $\mathbf{( 3 5 )}$ & $\mathbf{( 2 5 )}$ \\
\hline weniger als 1 Stunde & 11 & 12 & 14 & 10 \\
1 bis 3 Stunden & 13 & 12 & 19 & 14 \\
3 bis 5 Stunden & 4 & 1 & 1 & 1 \\
5 bis 10 Stunden & 2 & 1 & 1 & 0 \\
mehr als 10 Stunden & 0 & 0 & 0 & 0 \\
\hline
\end{tabular}

Ein wichtiges Ziel ist deshalb, dass die Zeit außerhalb des Unterrichts bewusst genutzt werden soll, um Lernfortschritte zu erzeugen. Der Unterricht alleine, ob lernzentriert oder lehrerzentriert gestaltet, kann nur begrenzten Einfluss auf das Erlernen einer Fremdsprache haben. Wenn es der Lehrperson gelingt, die Lernenden zur Beschäftigung mit der Fremdsprache außerhalb des Unterrichts anzuregen, fördert das den Fortschritt und die Motivation der Lernenden zusätzlich. Dazu gibt es heutzutage viele, insbesondere digitale Möglichkeiten, Unterricht außerhalb eines Klassen-zimmers zu gestalten. Auch die Antworten zur Frage 13 aus der Befragung zum Einsatz der Sprachlernspiele im SS 2017 zeigen, dass die Lernenden Interesse für digitale Lern- und Lehrmethoden haben (siehe Anhang 22). Während Sprachlernspiele im Unterricht Interaktivität, Motivation und Kommunikation fördern, sollen Methoden wie „Flipped Classroom" eingesetzt werden, um das Lernen auf den Raum und die Zeit außerhalb des Klassenzimmers zu erweitern. Eine sinnvolle Kombination von Sprachlernspielen mit digitalen Lehr- und Lernmethoden bedarf noch weiterer empirischer Untersuchungen.

Wegen der Faktorenkomplexion des Fremdsprachenunterrichts wird das untersuchte Phänomen durch mehr Variablen gleichzeitig beeinflusst. Auch die Dynamik, die Instabilität sowie hohe Individualität von Prozessen und Produkten des Lehrens und Lernens können die Ergebnisse des Quasi-Experiments beeinflussen (vgl. Riemer 2014, S. 25). Die Übertragbarkeit der Forschungsergebnisse ist daher eingeschränkt.

Außerdem ist die Anzahl der Probanden (Experimentalgruppe 30, Kontrollgruppe 36) nicht ideal für die quantitative Analyse und deren Ergebnisse (zur Probanden-Information siehe Kapitel 4.2.2). Der Einfluss der Gruppenstärke wird im Kapitel 5.4 ausführlich diskutiert. Die Größe der Gruppen bedeutet auch, dass die gleiche Methode im mittleren oder großen Großgruppenunterricht 
nicht unbedingt geeignet ist. Um die Übertragbarkeit der Forschungsergebnisse zu verbessern, wäre es wünschenswert, mehr Replikationsstudien in der Zukunft unter verschiedenen Rahmenbedingungen durchzuführen.

Die Kommunikationskompetenz der Lernenden kann in dieser Arbeit nur am Rand behandelt werden, weil mündliche Prüfungen "ressourcenintensiv" und deren praktische Durchführung organisatorisch anspruchsvoll ist (vgl.Eberharter et al. 2018, S. 98). Es gibt keine validen Instrumente für eine objektive Evaluation der kommunikativen Kompetenzen und Aussprache der Lernenden, die leicht einsetzbar und zielgruppengeeignet wären. Entsprechend könnte es von Bedeutung für die Sprachlernforschung sein, passende Evaluationsinstrumente für die Kommunikationskompetenz zu entwickeln.

Der genderbezogene Unterschied beim Vorzug von Sprachlernspielen kann nicht erfasst werden, weil beide Gruppen nur sehr geringe Anzahl von Frauen aufweisen. Es ist allerdings durchaus möglich, dass Frauen und Männer verschiedene Spieltypen bevorzugen. Darüber hinaus können Studierende aus verschiedenen Fachrichtungen verschiedene Spielgewohnheiten zeigen. Weitere Untersuchungen in diesen Bereichen können neue Einsichten für den Einsatz der Sprachlernspiele bringen.

Die vorliegende Forschung zeigt, dass es zur Optimierung des studienbegleitenden DaF-Unterrichts weiterer Forschungen bedarf. Der studienbegleitende Fremdsprachenunterricht ist von der spezifischen Zielgruppe und den spezifischen Rahmenbedingungen jeder Hochschule stark abhängig. Das Curriculum und die Unterrichtsorganisation müssen von jeder Hochschule nach den Bedürfnissen der jeweiligen Zielgruppe entworfen werden. Darüber hinaus zeigt sich in der regionalen Vielfalt von Fremdsprachenausbildungen in China, dass die Erarbeitung eines nationalen Standards nicht unbedingt adäquat ist. Forschung zu den Themen nationaler Prüfungen, Lehrwerkentwicklungen und Curricula sind für die zukünftige Weiterentwicklung des studienbegleitenden DaF-Unterrichts von Bedeutung. Außerdem sollte man spielerische Elemente in chinesischen DaF-Lehrwerken einführen, sodass Lehrende und Lernende leichten Zugang zum spielerischen Lehren und Lernen finden können.

An dieser Stelle soll betont werden, dass die vorliegende Arbeit nicht versucht, die Funktion und Wirkung von Sprachlernspielen im Fremdsprachenunterricht zu verallgemeinern. Sprachlernspiele sind kein Wundermittel: Einerseits ist das motivierende Potenzial einer Lehrmethode wegen der Individualität und Komplexität von Motivation nicht generell vorhersagbar (vgl. Riemer 2016, S. 269). Andererseits kommt es auch auf die Fähigkeiten und Einstellung der Lehrperson an, wie sie ihren Unterricht gestalten kann und will. Um die Unterrichtsqualität zu verbessern, könnten aber beispielsweise mehr Fort- und 
Ausbildungsmöglichkeiten für Lehrende angeboten werden. Den Lehrenden soll bewusst gemacht werden, dass man durch passende Methoden den Unterrichtsverlauf und -erfolg verändern kann, und dass die Unterrichtspraxis kritisch hinterfragt werden muss, wenn man die Unterrichtsqualität verbessern will. Unterrichtsmethoden sollen immer zielgruppengeeignet eingesetzt werden. Unterrichtsforschung bietet die einzige Möglichkeit, die Zielgruppe richtig kennen zu lernen.

Abschließend möchte ich auf das Thema Lehrerzentriertheit vs. Lernerzentriertheit eingehen. Da die vorliegende Arbeit eine Aktionsforschung enthält, bin ich gleichzeitig die Forscherin, die Lehrerin und auch die Erforschte. Die Erfahrung mit Sprachlernspielen hat mir bewusst gemacht, dass Lehrerzentriertheit und Lernerzentriertheit keinen Widerspruch darstellen. Wenn die Lehrperson die Lernenden in das Zentrum des Unterrichts stellt, kann die Lehrperson trotzdem weiterhin eine zentrale Rolle im Unterricht spielen. Außerdem bringt lehrerzentrierter Unterricht eine Gefahr mit sich, dass die Lernenden am Unterricht nicht teilnehmen und damit nicht durch Unterricht erreicht werden können. Die Forschung von John Hattie (2010) macht deutlich, dass die Lehrperson die wichtigste Rolle im Unterricht spielt und spielen sollte. Mit einem Zitat aus seinem Werk beende ich die vorliegende Diskussion:

„... (to invoke) the power of developing a warmer socio-emotional climate in the classroom and fostering effort and thus engagement for all students. Requires teachers to enter the classroom with certain conceptions about progress, relationships and students. It requires them to believe that their role is that of a change agent - that all students can learn and progress, that achievement for all is changeable and not fixed, and that demonstrating to all students that they care about their learning is both powerful and effective" (Hattie 2010, S. 128). 



\begin{abstract}
:
Das Ziel der vorliegenden Dissertation ist, die Einsetzbarkeit und die Wirksamkeit der Sprachlernspiele im DaF-Unterricht an chinesischen Universitäten empirisch zu überprüfen. Dazu wird eine einjährige Aktionsforschung durchgeführt. Eine Reihe von verschiedenen Datenerhebungsmethoden wurden verwendet, um ein möglichst umfassendes Bild von Sprachlernspielen zu liefern: Befragungen, Tests, Unterrichtsbeobachtungen sowie Lehrtagebücher wurden quantitativ und qualitativ analysiert und ausgewertet. Die Ergebnisse bestätigen zum großen Teil die Wirkungen von Sprachlernspielen auf Motivation und Lernfortschritt. Sorgfältige Vorbereitung und kreative Herangehensweise können dabei helfen, Sprachlernspiele im DaF-Unterricht in China als festen Unterrichtsbestandteil einzusetzen. Die Dissertation ist besonders für die Fremdsprachenlehrenden interessant, die ihren Fremdsprachenunterricht aktiver gestalten möchten.
\end{abstract}

\title{
Abstract (English):
}

This study aims to explore the applicability and effectiveness of game-like activities in German as foreign language classrooms at Chinese universities. For this purpose, the researcher conducted a one-year action research. In order to provide a comprehensive picture of game-like activities, the data was collected through different methods: Surveys, tests, classroom observations and teaching diaries. The data was analysed and evaluated quantitatively and qualitatively. The results confirm that game-like activities may have large effects on students' motivation and learning outcomes. With the help of careful preparation and creativities, game-like activities can be a regular approach in German as foreign language classrooms in China. The present study is particularly interesting for foreign language teachers, who wish to create a more active foreign language classroom. 



\section{Abbildungsverzeichnis}

Abbildung 1: Heuristic Model of Variables Influencing WTC (Macintyre et al. 1998, S. 547)

Abbildung 2: Drei Möglichkeiten der Begriffserklärung nach Klippel (1980b)

Abbildung 3: Die sechs Merkmale des Spiels im Scheuerls Konzept ......... 51

Abbildung 4: Zuordnung vom Sprachlernspiel von Bohn und Schreiter (vgl. Bohn und Schreiter 2001, S. 419f.)

Abbildung 5: Hierarchische Beziehung zwischen den Begriffen didaktisches Spiel, Unterrichtsspiel und Sprachlernspiel (vgl. Mátyás 2009, S. 93)

Abbildung 6: Kriterien des Sprachlernspiels nach Kleppin von Mátyás (2009, S. 94)

Abbildung 7: Die miteinander verknüpften Merkmale

Abbildung 8: Der Kreislauf von Aktion und Reflexion (Altrichter und Posch 1998, S. 17)

Abbildung 9: $\quad$ Überblick des Plans WS 2016-17 ............................................. 113

Abbildung 10: Übersicht der Datenerhebungsmethoden im WS 2016-17 ... 114

Abbildung 11: Streudiagramm von Gesamtnote der Klausur 1 WS 2016-17

Abbildung 12: Die Ergebnisse von Item 6 der Befragung zum Einsatz der Sprachlernspiele WS 2016-17

Abbildung 13: Übersicht der Datenerhebungsmethoden SS 2017

Abbildung 14: Die Ergebnisse von Item 6 der Befragung zum Einsatz der Sprachlernspiele SS 2017

Abbildung 15: Ergebnisse von Item 7 der Befragung zum Einsatz der Sprachlernspiele SS 2017

Abbildung 16: Ergebnisse von Item 11 der Befragung zum Einsatz der Sprachlernspiele SS 2017

Abbildung 17: Entwicklungsprozess einer neuen Aktivitätsform

Abbildung 18: Antworten zum bevorzugten Spieltyp WS 2016-17 .............. 178

Abbildung 19: Antworten zum bevorzugten Spieltyp SS 2017 



\section{Tabellenverzeichnis}

Tabelle 1: Ausgewählte Daten zur Situation Deutschlernen in China 2015 (vgl. Auswärtiges Amt 2015, S. 12)

Tabelle 2: Die Problemfelder des Großgruppenunterrichts (Winkler 2011, S. 3)

Tabelle 3: Die sechs Merkmale eines Spiels vom Scheuerl und deren Interpretationen

Tabelle 4: Gegenüberstellung des Kleppin-Konzepts zum Scheuerlkonzept nach Mátyás (2009, S. 101f.)

Tabelle 5: Die Prototypische Gegenüberstellung von Übung und Aufgabe (Huneke und Steinig 2013, S. 233)

Tabelle 6: Gruppenstärken der Klassen vor und nach der Umstrukturierung

Tabelle 7: Zahl der Anwesenden und der Beteiligten bei der Befragung zur Lernerbiographie 102

Tabelle 8: Übersicht der Befragung zur Lernbiografie 102

Tabelle 9: Überblick über Gruppenverteilung und Lernmaterialien WS 2016-17

Tabelle 10: Kriterien und Itemüberblick der Befragung zur

Lernmotivation und Unterrichtsevaluation WS 2016-17

Tabelle 11: Kriterien und Itemüberblick der Befragung zum Einsatz von Sprachlernspielen WS 2016-17

Tabelle 12: Überblick der Items mit signifikantem Unterschied von der Befragung zur Motivation und Unterrichtsevaluation WS 2016-17

Tabelle 13: Ergebnisse vom MWU-Test von Item 2 der Befragung zur Motivation und Unterrichtsevaluation WS 2016-17

Tabelle 14: Ergebnisse vom MWU-Test von Item 2 am Anfang und Ende des Semesters WS 2016-17

Tabelle 15: Ergebnisse vom MWU-Test von Item 9 im WS 2016-17

Tabelle 16: Ergebnisse vom MWU-Test des Englischeinstufungstests

Tabelle 17: Übersicht der Notenverteilung der Semesterprüfung WS 2016-17

Tabelle 18: Ergebnisse vom MWU-Test der Klausur 1 121

Tabelle 19: Ergebnisse vom MWU-Test der Klausur 2

Tabelle 20: Ergebnisse vom MWU-Test der Teilfertigkeiten WS 2016-17 .... 121

Tabelle 21: Übersicht des Index lexikalischer Vielfalt der 
Tabelle 22: Übersicht der Ergebnisse von Item 1 und 2 von der Befragung zum Einsatz der Sprachlernspiele WS 2016-17 124

Tabelle 23: Übersicht der Ergebnisse von Item 3 von der Befragung zum Einsatz der Sprachlernspiele WS 2016-17

Tabelle 24: Übersicht der Ergebnisse von Item 7 von der Befragung zum Einsatz der Sprachlernspiele WS 2016-17

Tabelle 25: Übersicht der Ergebnisse von Item 8 von der Befragung zum Einsatz der Sprachlernspiele WS 2016-17 126

Tabelle 26: Übersicht der Begründungen von Item 10 von der Befragung zum Einsatz der Sprachlernspiele WS 2016-17

Tabelle 27: Übersicht der Items mit signifikantem Unterschied von der Befragung zur Motivation und Unterrichtsevaluation SS 2017 .... 131

Tabelle 28: Ergebnisse von MWU-Tests von Items aus Tabelle 27 131

Tabelle 29: Vergleich der Mittelwerte von Item 1.3 WS 2016-17 und SS 2017

Tabelle 30: Ergebnisse vom MWU-Test von Item 9 im SS 2017 .................... 133

Tabelle 31: Übersicht der Notenverteilung der Semesterprüfung SS 2017 ... 133

Tabelle 32: Ergebnisse von MWU-Tests der Semesterprüfung SS 2017 ....... 134

Tabelle 33: Ergebnisse von MWU-Tests der Teilfertigkeiten SS 2017 ........... 134

Tabelle 34: Übersicht des Index lexikalischer Vielfalt der Semesterprüfung SS 2017 135

Tabelle 35: Ergebnisse von MWU-Tests von C-Tests SS 2017

Tabelle 36: Ergebnisse von MWU-Tests zwei Semestern von Item 1 und 2 .. 138

Tabelle 37: Ergebnisse von MWU-Tests zum Vergleich der Funktionen der Sprachlernspiele 138

Tabelle 38: Übersicht der Ergebnisse von Item 8 und 12 ................................ 141

Tabelle 39: Übersicht der Kommentare von Item 10 und 12 .......................... 142

Tabelle 40: Überblick vom Beobachtungsbogen B .......................................... 145

Tabelle 41: Items mit signifikantem Unterschied zu Unterrichtsdesign und Vorbereitung ............................................................................ 145

Tabelle 42: Übersicht der Ergebnisse von MWU-Tests Item 5,6 und 7 ......... 145

Tabelle 43: Items mit signifikantem Unterschied zum Verlauf des Unterrichts 146

Tabelle 44: Übersicht der Ergebnisse der MWU-Tests von Items zum Verlauf des Unterrichts 146

Tabelle 45: Items mit signifikantem Unterschied zur Effizienz des Unterrichts 147

Tabelle 46: Übersicht der Ergebnisseder MWU-Tests von Items zur Effizienz des Unterrichts

Tabelle 47: Übersicht der Ergebnisse von MWU-Test von Item 41 148 
Tabelle 48: Kodierung von Textsegmenten des Lehrtagebuchs WS 2016-17

Tabelle 49: Kodierung von Textsegmenten des Lehrtagebuchs SS 2017 ....... 153

Tabelle 50: Überblick der eingesetzten Datenerhebungsmethoden und der gewonnenen Daten WS 2016-17 ............................................ 155

Tabelle 51: Überblick der eingesetzten Datenerhebungsmethoden und der gewonnenen Daten SS 2017 .................................................. 156

Tabelle 52: Antworten der Forschungsfragen zur Motivationserhöhung ..... 156

Tabelle 53: Antworten der Forschungsfragen zur Verbesserung der sprachlichen Kompetenzen

Tabelle 54: Ergebnisse von MWU- Tests der Semesterprüfungen im Studienjahr 2017-18

Tabelle 55: Ergebnisse von MWU-Tests über die Aktivitätsdauer WS 2016-17 und SS 2017

Tabelle 56: Textsegmentenanzahl zu den ungelungenen organisatorischen Problemen

Tabelle 57: Wichtige Reflexionen aus dem Lehrtagebuch zur organisatorischen Methode WS 2016-17

Tabelle 58: Wichtige Reflexionen aus dem Lehrtagebuch zur organisatorischen Methode SS 2017

Tabelle 59: Beispiel der Items zum eingesetzten Spielen 177

Tabelle 60: Ergebnisse von „Kruskal -Wallis H“ Tests WS 2016-17

Tabelle 61: Ergebnisse von „Kruskal -Wallis H“ Tests SS 2017

Tabelle 62: Zusammenfassung der Begründungen zur Sozialform der Sprachlernspiele 179

Tabelle 63: Übersicht der Lernzeit außerhalb des Unterrichts 



\section{Literaturverzeichnis}

Aguado, Karin (2014): Triangulation. In: Jutta Settinieri (Hg.): Empirische Forschungsmethoden für Deutsch als Fremd- und Zweitsprache. Paderborn: Schöningh (UTB Sprachwissenschaft, 8541), S. 47-55.

Agudo, Juan de Dios Martinez (2018): Introduction and Overview. In: Agudo, Juan de Dios Martínez (Hg.): Emotions in Second Language Teaching. Theory, Research and Teacher Education. Cham: Springer International Publishing, S. 1-13.

Altrichter, Herbert (2007): Lehrerinnen und Lehrer erforschen ihren Unterricht: Unterrichtsentwicklung und Unterrichtsevaluation durch Aktionsforschung. Unter Mitarbeit von Peter Posch, Pädagoge und 1938-. 4., überarb. und erw. Aufl. (Bis 3. Auflage Altrichter, Herbert Lehrer erforschen ihren Unterricht).

Altrichter, Herbert; Posch, Peter (1998): Lehrer erforschen ihren Unterricht. Eine Einführung in die Methoden der Aktionsforschung. 3., durchges. und erw. Bad Heilbrunn: Verlag Julius Klinkhardt.

Aubrey, Scott (2011): Facilitating Interaction in East Asian EFL Classrooms: Increasing Students' Willingness to Communicate. In: Literature Education in Asia 2 (2), S. 237-245.

Auswärtiges Amt (2015): Deutsch als Fremdsprache weltweit. Datenerhebung 2015. Online verfügbar unter https://www.daad.de/medien/der-daad/unsere-aufgaben/deutsche-sprache/pdfs/daad_germanistik_netzwerk-deutsch_ datenerhebung_2015.pdf, zuletzt geprüft am 23.01.2017.

Bausch, Karl-Richard (Hg.) (2007): Handbuch Fremdsprachenunterricht. 5. Aufl. Tübingen: Francke (UTB Pädagogik, Sprachwissenschaften, 8042/8043).

Becker-Sliwa, Katja (2017): Kugellager in Wuhan. Lernaktivierung an chinesischen Schulen. In: Fremdsprache Deutsch (56), S. 46.

Behme, Helma (1985a): Mit dem Spiel fängt alles an... Ein methodischer Beitrag zur Gestaltung des Unterrichtsbeginns mit Sprechspielen. In: Informationen Deutsch als Fremdsprache (1), S. 31-37.

Behme, Helma (1985b): Miteinander reden lernen, Sprechspiele im Unterricht. München: Iudicium-Verl. (Studium Deutsch als Fremdsprache - Sprachdidaktik, 4).

Boeckmann, Klaus-Börge (2016): Aktionsforschung. In: Eva Burwitz-Melzer, Grit Mehlhorn, Claudia Riemer, Karl-Richard Bausch und Hans-Jürgen Krumm (Hg.): Handbuch Fremdsprachenunterricht. 6., völlig überarbeitete und erweiterte Auflage. Tübingen: A. Francke (UTB, 8043), S. 592-596. 
Bohn, Rainer; Schreiter, Ina (2001): Sprachlernspiele. In: Gert Henrici und Claudia Riemer (Hg.): Einführung in die Didaktik des Unterrichts Deutsch als Fremdsprache mit Videobeispielen. 3. Aufl. 2 Bände. Baltmannsweiler: Schneider Hohengehren (Perspektiven Deutsch als Fremdsprache, 1), S. 419-436.

Brede, Julia Ricart (2014): Beobachtung. In: Jutta Settinieri (Hg.): Empirische Forschungsmethoden für Deutsch als Fremd- und Zweitsprache. Paderborn: Schöningh (UTB Sprachwissenschaft, 8541), S. 137-146.

Cao, Yiqian; Philp, Jenefer (2006): Interactional context and willingness to communicate: A comparison of behavior in whole class, group and dyadic interaction. In: System 34 (4), S. 480-493. DOI: 10.1016/j.system.2006.05.002.

Cao, Zhijuan (2015): Die Ist- Analyse der Gradientenwicklung von Hochschulbildung in Provinz Yunnan und deren Bedeutung. In: Education Teaching Forum (13), S. 13-14.

Caspari, Daniela (2016): Sprachenlehren als Beruf. In: Eva Burwitz-Melzer, Grit Mehlhorn, Claudia Riemer, Karl-Richard Bausch und Hans-Jürgen Krumm (Hg.): Handbuch Fremdsprachenunterricht. 6., völlig überarbeitete und erweiterte Auflage. Tübingen: A. Francke (UTB, 8043), S. 305-311.

Caspari, Daniela; Helbig, Beate; Schmelter, Lars (2007): Forschungsmethoden: Explorativ-interpretatives Forschen. In: Karl-Richard Bausch (Hg.): Handbuch Fremdsprachenunterricht. 5. Aufl. Tübingen: Francke (UTB Pädagogik, Sprachwissenschaften, 8042/8043), S. 499-505.

Casper-Hehne, Hiltraud (2015): Interkulturelle deutsch-chinesische Wissenschaftskommunikation. In: Wenjian Jia und Yulu Mou (Hg.): Sprache als Brücke der Kulturen. Sprachpolitik und Sprachwirklichkeit in Deutschland und China. 1. Aufl. Beijing: Verlag für Foreign Language Teaching and Research, 152-168.

Chang, Jinxiong; Ruan, Tiancheng; Chang, Dawei (2018): Der Einfluss von Erweiterung der Hochschulzulassung auf die Bildungsgleichheit zwischen Stadt und Land. In: Academic Research (7), S. 78-86.

Chou, Mei-Wu (2015): Grammatiklernen und -lehren im universitären DaFUnterricht Taiwans. Eine empirische Studie zu didaktischen, sprachlichen und kulturellen Aspekten. Tübingen: Narr (Giessener Beiträge zur Fremdsprachendidaktik).

Cuéllar, Lourdes; Oxford, Rebecca L. (2018): Language teachers' emotions: emerging from the shadows. In: Agudo, Juan de Dios Martínez (Hg.): Emotions in Second Language Teaching. Theory, Research and Teacher Education. Cham: Springer International Publishing, 53-72. 
Dauvillier, Christa; Lévy-Hillerich, Dorothea (2008): Spiele im Deutschunterricht. [Nachdr.]. Berlin: Langenscheidt (Fernstudienprojekt zur Fort- und Weiterbildung im Bereich Germanistik und Deutsch als Fremdsprache, Fernstudieneinheit 28).

Dauvillier, Christa; Meese, Herrad (2004): Spiele im Deutschunterricht. Berlin, München: Langenscheidt (Fernstudieneinheit, 28).

Dewaele, Jean-Marc; Gkonou Christina; Mercer, Sarah (2018): Do ESL/EFL teachers' emotional intelligence, teaching experience, proficiency and gender affect their classroom practice? In: Agudo, Juan de Dios Martínez (Hg.): Emotions in Second Language Teaching. Theory, Research and Teacher Education. Cham: Springer International Publishing, 125-141.

Die Regierung der Provinz Yunnan (2017a): Bevölkerung und Nationen der Provinz Yunnan. 人口及民族. Online verfügbar unter http://www.yn.gov.cn/ yn_yngk/gsgk/201509/t20150923_22230.html, zuletzt geprüft am 22.02.2019.

Die Regierung der Provinz Yunnan (2017b): Sprachen der Provinz Yunnan. 语言 文字. Online verfügbar unter http://www.yn.gov.cn/yn_yngk/gsgk/201509/ t20150923_22231.html, zuletzt geprüft am 22.02.2019.

Döring, Nicola; Bortz, Jürgen (2016): Forschungsmethoden und Evaluation in den Sozial- und Humanwissenschaften. Unter Mitarbeit von Sandra Pöschl. 5. vollständig überarbeitete, aktualisierte und erweiterte Auflage. Berlin, Heidelberg: Springer (Springer-Lehrbuch).

Döring, Sabine (1997): Lernen durch Spielen. Spielpädagogische Perspektiven institutionellen Lernens. 1. Aufl. Weinheim: Dt. Studien-Verl.

E, Fan (2013): Untersuchung von Bildung in Westchina: Grundausbildung muss dringend verbessert werden. 西部教育调查: 最需提高的仍是义务教育. In: Insight China (7), S. 60-63.

Eberharter, Kathrin; Kremmel, Benjamin; Konzett-Firth, Carmen (2018): Produktive Fertigkeiten überprüfen und bewerten. In: Barbara Hinger und Wolfgang Stadler (Hg.): Testen und Bewerten fremdsprachlicher Kompetenzen. Tübingen: Narr Francke Attempto (Narr Studienbücher), S. 87-115.

Elliott, John (1991): Action research for educational change. Milton Keynes [England], Philadelphia: Open University Press (Developing Teachers and Teaching).

Fei, Liangjun; Zi, Linbo; Gan, Shu; Bai, Yunfang (2016): Geographische Merkmale von Provinz Yunnan und deren Konsequenzen. 云南地理国情普查特 点及其成果应用分析探讨. In: Geomatics Technology and Equipment (3), S. 67-69.

Feldmeier, Alexis (2014): Besondere Forschungsansätze: Aktionsforschung. In: Jutta Settinieri (Hg.): Empirische Forschungsmethoden für Deutsch als 
Fremd- und Zweitsprache. Paderborn: Schöningh (UTB Sprachwissenschaft, 8541), S. 255-267.

Feng, Jun; Zhang, Min; Ou. Ying (2013): Bedarfsanalyse von Fremdsprachenpersonal in Yunnan im Rahmen von nationaler Strategie „Brückenkopf“. 云南 “桥头堡” 战略下外语人才需求调查问卷统计分析. In: Shang Qing Journal (20), 28, 145.

Friedrich, Thorsten; Jan, Eduard von (1990): Lernspielekartei. Spiele und Aktivitäten für einen kommunikativen Sprachunterricht. 1. Aufl., 3. Dr. München: Hueber.

Gass, Susan; Mackey, Alison (2012): Data Elicitation for Second and Foreign language Research. 1. Aufl. Beijing: Foreign Language Teaching and Research Press.

Gerbig, Johannes J. (2007): Vergleich der Situation in der Volksrepublik China in Ihren frühreren Grenzen, in Hongkong und in Taiwan. In: Ulrich Ammon, Roswitha Reinbothe und Jianhua Zhu (Hg.): Die deutsche Sprache in China: Geschichte, Gegenwart, Zukunftsperspektiven, S. 311-321.

Goh, Renate (2017): Der Weg zum Ziel. Unterricht planen für große Gruppen. In: Fremdsprache Deutsch (56), S. 31-36.

Goldberger, Josef (2010): Deutschunterricht an chinesischen Universitäten. Eine Beschreibung der Situation und der Entwicklungstendenzen. Online verfügbar unter https://www.researchgate.net/profile/Josef_Goldberger/publication/28736 1037_Deutschunterricht_an_chinesischen_Universitaten_Eine_Beschreibung der_Situation_und_der_Entwicklungstendenzen/links/5676175508ae502c99 ce0f87/Deutschunterricht-an-chinesischen-Universitaeten-Eine-Beschrei bung-der-Situation-und-der-Entwicklungstendenzen.pdf, zuletzt geprüft am 20.10.2018.

Grätz, Ronald (2001): Vom Spielen, Leben, Lernen. In: Fremdsprache Deutsch (25), S. 5-8.

Groos, Karl (1991): Der Lebenswert des Spiels. In: Hans Scheuerl (Hg.): Das Spiel. Theorien des Spiels. 10. Aufl. Weinheim: Beltz (Berlz Studienbuch, Bd. 2).

Grotjahn, Rüdiger (1995): Der C-Test. State of the Art. In: Zeitschrift für Fremdsprachen 6 (2), 37-60.

Grotjahn, Rüdiger (2007): Konzepte für die Erforschung des Lehrens und Lernens fremder Sprachen: Forschungsmethodologischer Überblick. In: Karl-Richard Bausch (Hg.): Handbuch Fremdsprachenunterricht. 5. Aufl. Tübingen: Francke (UTB Pädagogik, Sprachwissenschaften, 8042/8043), S. 493-499.

Grum, Urska (2012): Anwendungsbeitrag: Anwendungsbeispiele statistischer Verfahren zur Analyse von Lernersprachdaten. In: Sabine Doff (Hg.): Fremdsprachenunterricht empirisch erforschen. 1. Aufl. Tübingen: Narr, S. 274-285. 
Gu, Lijuan; Lü, Xueling; Zhou, Zhengan (2003): Einblick in den Unterrichtsalltag Deutsch als Wahl- und Nebenfach in der VR China am Beispiel der Univerisität Zhejiang, der Polytechnischen Universität Harbin und der Univeristät Hunan. In: Ulrich Wannagat (Hg.): Deutsch als zweite Fremdsprache in Ostasien - neue Perspektiven. [... internationales Symposium ... vom 27. - 30. April 1999 am Chung Chi College der Chinesischen Universität von Hongkong]. Tübingen: Stauffenburg (Arbeiten zur angewandten Linguistik, 2), S. 87-96.

Gu, Shiyuan (2004): DaF in China. Curricula, Unterrichtsmethoden und Konsequenzen für Lehraus- und -fortbildung. In: Hans-Werner Hess (Hg.): Didaktische Reflexionen. „Berliner Didaktik“ und Deutsch als Fremdsprache heute. Tübingen: Stauffenburg (Arbeiten zur angewandten Linguistik, 3), S. 89-95.

Han, Wei (2006): Warum ist der Mund schwer aufzumachen? Eine empirische Untersuchung der Sprechunfähigkeit der chinesischen Deutschlerner unter soziokulturellem und psychologischem Aspekt. In: Jianhua Zhu und Siegfried Grosse (Hg.): Interkulturelle Kommunikation Deutsch - Chinesisch. Kolloquium zu Ehren von Siegfried Grosse, 25.11. - 27.11.2004, Shanghai (19), S. 407-418.

Hansen, Maike; Wendt, Michael (1990): Sprachlernspiele. Grundlagen und annotierte Auswahlbibliographie unter besonderer Berücksichtigung des Französischunterrichts. Tübingen: Narr.

Hargreaves, Andy (2000): Mixed emotions: Teachers' perceptions of their interactions with students. In: Teaching and teacher education 16 (8), S. 811-826.

Haß, Frank (2017a): Differenzierung. In: Carola Surkamp (Hg.): Metzler Lexikon Fremdsprachendidaktik. Stuttgart: J.B. Metzler.

Haß, Frank (2017b): Sozialform. In: Carola Surkamp (Hg.): Metzler Lexikon Fremdsprachendidaktik. Stuttgart: J.B. Metzler, S. 306-309.

Hattie, John (2010): Visible learning. A synthesis of over 800 meta-analyses relating to achievement. Reprinted. London: Routledge.

He, Xiangdong (2008): Kultur von Westchina. Kulturelle Ressource und Bildung. 中国西部人文: 文化资源与素质教育——点燃西部的阳光. 1. Aufl. Beijing: Verlag der Reming Universität China.

Helmke, Andreas (2017): Unterrichtsqualität und Lehrerprofessionalität. Diagnose, Evaluation und Verbesserung des Unterrichts: Franz Emanuel Weinert gewidmet. Unter Mitarbeit von Franz E. Weinert. 7. Auflage. Seelze-Velber: Klett/Kallmeyer (Schule weiterentwickeln, Unterricht verbessern Orientierungsband).

Hernig, Marcus (2010): Deutsch in China. In: Hans-Jürgen Krumm, Gerold Ungeheuer und Herbert Ernst Wiegand (Hg.): Handbücher zur Sprach- und 
Kommunikationswissenschaft. [Neubearb.]. Berlin [u.a.]: de Gruyter Mouton (35.2), S. 1637-1642.

Hess, Hans Werner (2001): Deutschunterricht und Germanistikstudium in China. In: Gerhard Helbig, Lutz Götze, Gert Henrici und Hans-Jürgen Krumm (Hg.): Deutsch als Fremdsprache Ein internationale Handbuch. 2. Halbband. Berlin: de Gruyter (Handbücher zur Sprach- und Kommunikationswissenschaft, 19,2), S. 1579-1586.

Hess, Hans-Werner (1992): „Die Kunst des Drachentötens“. Zur Situation von Deutsch als Fremdsprache in der Volksrepublik China. Diss., Berlin.

Hess, Natalie (2007): Teaching large multilevel classes. 8. Aufl. Cambridge: Cambridge University Press (Cambridge handbooks for language teachers).

Hesse, Hermann-Günter (2007): Lernen innerhalb und außerhalb der Schule aus interkultureller Perspektive. In: Gisela Trommsdorff und Niels-Peter Birbaumer (Hg.): Anwendungsfelder der kulturvergleichenden Psychologie. Göttingen: Hogrefe (Enzyklopädie der Psychologie Theorie und Forschung Kulturvergleichende Psychologie, Bd. 3), S. 187-277.

Hoppe, Hans (1983): Spiel im Deutsch- und Fremdsprachenunterricht. Begründungen und Kriterien für die fächerspezifische Spielauswahl und -verwendung. In: Karl Josef Kreuzer (Hg.): Handbuch der Spielpädagogik. 1. Aufl. 2 Bände. Düsseldorf: Schwann (Spiel im frühpädagogischen und schulischen Bereich, 2), S. 285-303.

Hoppe, Hans (1985): Spieldidaktische Kriterien und Verfahrensvorschläge für den Einsatz von Sprachlernspielen im Fremdsprachenunterricht mit Erwachsenen. In: Informationen Deutsch als Fremdsprache (1), S. 4-14.

Hu, Dexin (2017): Regional-vergleichende Untersuchung zur Bildungsgleichheit zwischen Stadt und Land in VR China. 我国城乡教育公平程度的区域比较 研究. In: Contemporary Education Science (3), S. 14-18.

$\mathrm{Hu}$, Jianming (2003): Entwicklung und Zustand der Grundausbildung vom Englisch in Westchina. 我国西部基础英语教育的现状与发展. In: Foreign Language Teaching in School (Middle Version) (3), S. 14-18.

$\mathrm{Hu}$, Wenzhong (2001): A matter of balance - Reflections on China's foreign language policy in education. 我国外语教育规划的得与失. In: Foreign Language Teaching and Research 33 (4), S. 243-251.

Huneke, Hans-Werner; Steinig, Wolfgang (2010): Deutsch als Fremdsprache. Eine Einführung. 5., neu bearb. und erw. Aufl. Berlin: Erich Schmidt (Grundlagen der Germanistik, 34).

Huneke, Hans-Werner; Steinig, Wolfgang (2013): Deutsch als Fremsprache. Eine Einführung. 6. Aufl. Berlin: Erich Schmidt. 
Hunold, Cordula (2009): Kulturspezifische Einflüsse der Lehr-und Lernumgebung auf die (Aussprache) leistungen chinesischer Deutschlernender. In: Kristina Peuschel und Jan P. Pietzuch (Hg.): Kaleidoskop der jungen DaF-/ DaZ-Forschung. Dokumentation zur zweiten Nachwuchstagung des Fachverbandes Deutsch als Fremdsprache. Göttingen: Universitätsverlag Göttingen (Materialien Deutsch als Fremdsprache, 1866-8283, Bd. 80), S. 49-66.

Jentges, Sabine (2007): Effektivität von Sprachlernspielen. Zur Theorie und Praxis des Spieleinsatzes im Deutsch-als-Fremdsprache-Unterricht. Univ., Diss.-Marburg, 2005. Baltmannsweiler: Schneider Hohengehren (Perspektiven Deutsch als Fremdsprache, 21).

Jia, Wenjian; Wei, Yuqing (Hg.) (2011): Forschungsbericht über Germanistik als Fach in China. 3. Aufl. Beijing: Foreign Language Teaching and Research Press.

Jiang, Mei; Chen, Liping; Ming, Zhu; Huang, Yanghong (2012): Die Merkmale vom Lernstil im Enligschunterricht von Studierenden aus dem Land an Universitäten in der Provinz Yunnan. 云南高校农村生源学生英语学习风格调 查研究. In: Education Teaching Forum (2), S. 76-78.

Jiang, Mei; Huang, Yanghong; Chen, Liping; Ming, Zhu (2011a): Einstellung und Motivation beim Englischlernen von Studierenden aus dem Land an Universitäten in der Provinz Yunnan. 云南高校农村生源学生英语学习态度和动 机的实证研究. In: Agricultural Education Research (2), 22-24, 7 .

Jiang, Mei; Huang, Yanghong; Ming, Zhu (2013): Lernsituation vom Englischlernen bei Studierenden aus dem Land an Universitäten in der Provinz Yunnan und passende Unterrichtsmethode. 云南高校农村生源学生英语学习现状 调查及教学策略研究. In: Huazhang (29), 189,193.

Jiang, Mei; Ming, Zhu; Quan, Hong; Chen, Liping (2011b): A Comparative Study of English Learning Strategies of College Students of Rural-Urban Enrollments: an Empirical Study in a University of Yunnan Province. 农村与城 市不同生源大学生英语学习策略对比研究-基于云南某高校的实证分析. In: China Agricultural Education (6), S. 75-78.

Jiang, Qiuxia (2012): A Study of Eco-context and Eco-compensations for FL Education in West China. 西部地区外语教育的生态语境及补偿机制研究. In: Foreign Language in China (6), S. 4-10.

Kacjan, Brigita (2008): Sprachelementspiele und Wortschatzerwerb im fremdsprachlichen Deutschunterricht mit Jugendlichen und jungen Erwachsenen. Maribor: Filozofska facultea.

Kacjan, Brigita (2010): Sprachlernspiele. In: Hans-Jürgen Krumm, Gerold Ungeheuer und Herbert Ernst Wiegand (Hg.): Handbücher zur Sprach- und Kommunikationswissenschaft. [Neubearb.]. Berlin [u.a.]: de Gruyter Mouton (35.2), S. 1177-1181. 
Kang, Su-Ja (2005): Dynamic emergence of situational willingness to communicate in a second language. In: System 33 (2), S. 277-292. DOI: 10.1016/j. system.2004.10.004.

Kieweg, Werner (2010): Übungsformen. In: Wolfgang Hallet und Frank G. König (Hg.): Handbuch Fremdsprachendidaktik. 1. Aufl. Seelze: Kallmeyer [u.a.], S. 182-186.

Kilp, Elóide (2003): Spiele für den Fremdsprachenunterricht. Aspekte einer Spielandragogik. Zugl.: Eichstätt, Kath. Univ., Diss., 2002. Tübingen: Stauffenburg-Verl. (Forum Sprachlehrforschung, 5).

Kleppin, Karin (1980): Das Sprachlernspiel im Fremdsprachenunterricht. Untersuchungen zum Lehrer- und Lernerverhalten in Sprachlernspielen. Tübingen: Narr (Tübinger Beiträge zur Linguistik, 152).

Kleppin, Karin (1989): Sprach- und Sprachlernspiele. In: Karl-Richard Bausch, Herbert Christ und Hans-Jürgen Krumm (Hg.): Handbuch Fremdsprachenunterricht. Tübingen: Francke; UTB (UTB), S. 185-187.

Kleppin, Karin (2007): Sprachspiele und Sprachlernspiele. In: Karl-Richard Bausch (Hg.): Handbuch Fremdsprachenunterricht. 5. Aufl. Tübingen: Francke (UTB Pädagogik, Sprachwissenschaften, 8042/8043), S. 263-266.

Kleppin, Karin (2017): Sprachlernspiele. In: Carola Surkamp (Hg.): Metzler Lexikon Fremdsprachendidaktik. Stuttgart: J.B. Metzler, S. 325-327.

Klippel, Friederike (1980a): Lernspiele im Englischunterricht. Mit 50 Spielvorschlägen. Zugl.: Dortmund, Päd. Hochsch., Diss., 1979 u.d.T.: Klippel, Friederike: Das Lernspiel und seine Verwendung im Englischunterricht, Teil 2. Paderborn, München: Schöningh (Informationen zur Sprach- und Literaturdidaktik, 34). Online verfügbar unter https://epub.ub.uni-muenchen. de/8645/1/8645.pdf, zuletzt geprüft am 13.12.2015.

Klippel, Friederike (1980b): Spieltheoretische und pädagogische Grundlagen des Lernspieleinsatzes im Fremdsprachenunterricht. Zugl.: T.1 von: Klippel, Friederike: Das Lernspiel und seine Verwendung im Englischunterricht / Zugl.: Dortmund, Univ., Diss., 1979. Frankfurt am Main: Peter D.Lang (/Europäische Hochschulschriften / 11], 93).

Klippel, Friederike (1995): Spielerische Lehrerfortbildung. In: Johannes-Peter Timm (Hg.): Ganzheitlicher Fremdsprachenunterricht. Weinheim: Dt. Studien-Verl. (19). Online verfügbar unter https://epub.ub.uni-muenchen. de/8765/1/8765.pdf, zuletzt geprüft am 12.12.2015.

Klippel, Friederike (1998): Spielen im Englischunterricht. In: Der fremdsprachliche Unterricht. Englisch 33 (35), S. 4-13. 
Klippel, Friederike (2010): Activities und Sprachlernspiele. In: Wolfgang Hallet und Frank G. König (Hg.): Handbuch Fremdsprachendidaktik. 1. Aufl. Seelze: Kallmeyer [u.a.], S. 186-190.

Koenig, Michael (2003): Nachdenken über Spiele. Ein Plädoyer für die spielerische Umgestaltung von Lernaktivitäten im Fremdsprachenunterricht. In: Babylonia (1), S. 8-17. Online verfügbar unter http://babylonia.ch/fileadmin/user_upload/documents/2003-1/Baby1_03Koenig.pdf, zuletzt geprüft am 31.01.2016.

Kong, Deming (2007): Die Hochschulen mit dem Fach Germanistik. In: Ulrich Ammon, Roswitha Reinbothe und Jianhua Zhu (Hg.): Die deutsche Sprache in China: Geschichte, Gegenwart, Zukunftsperspektiven, S. 123-140.

Königs, Frank G. (2017): Sprachlehrforschung. In: Carola Surkamp (Hg.): Metzler Lexikon Fremdsprachendidaktik. Stuttgart: J.B. Metzler, S. 322-324.

Krekeler, Christian (2011): Möglichkeiten und Grenzen der Wirkungsforschung in der Fremdsprachendidaktik am Beispiel der Fehlerkorrektur. In: Natalia Hahn und Thorsten Roelcke (Hg.): Grenzen überwinden mit Deutsch. 37. Jahrestagung des Fachverbands Deutsch als Fremdsprache an der Pädagogischen Hochschule Freiburg/Br. 2010, Bd. 85. Göttingen: Universitätsverlag (Materialien Deutsch als Fremdsprache), S. 209-221.

Kreuzer, Karl Josef (1983): Zur Komplexität der spielpädagogischen Fragestellungen und Bereiche. In: Karl Josef Kreuzer (Hg.): Handbuch der Spielpädagogik. 1. Aufl. 2 Bände. Düsseldorf: Schwann (Das Spiel unter pädagogischm, psychologischem und vergleichendem Aspekt, 1), S. 7-28.

Kube, Klaus (1977): Spieldidaktik. Zugl.: Hamburg, Univ., Diss., 1977 u.d.T.: Kube: Spieldidakrik für die Unterrichtspraxis. 1. Aufl. Düsseldorf: Schwann.

Li, Chuansong (2009): Die Geschichte von Fremdsprachenausbildung in der VR China. 新中国外语教育史. Beijing: Tourism Education Press.

Li, Chuansong; Xu, Baofa (2007): A History of Modern Foreign Language Education in China. 中国近现代外语教育史. Shanghai: Shanghai Foreign Language Education Press.

Li, Xin; Zhang, Yuqin; Li, Xuekun (2018): The Regional Difference of Poverty Population in Yunnan Province. 云南省贫困人口区域性差异研究. In: Journal of Yunnan Agricultural University(Social Science) 12 (1), S. 23-29.

Li, Yaling (2010): Zur Problematik der Gleichentwicklung von fremdsprachlicher Ausbildung an Grund- und Mittelschulen in China. 中国中小学外语教 育均衡发展的政策问题与对策. In: Foreign Language Teaching and Research in Basic Education (4), S. 33-37. 
Li, Yaling (2012): On the Development of Foreign Language Education Policies in China. 中国外语教育政策发展研究. 1. Aufl. Beijing: Verlag der Universität Beijing.

Li, Yuan; Lian, Fei (2017): Die deutsche Sprache in China: eine aktuelle Bestandsaufnahme. In: Jahrbuch für Internationale Germanistik 49 (2), S. 115-143.

Liang, Jun (2018): Analyse und Anwendung der spielerischen Übungen im Lehrwek "Studienweg Deutsch“. 《当代大学德语》教材中游戏类练习的 内容分析及其应用研究. In: Journal of Kaifeng Institue of Education 38 (3), S. $102-103$.

Liang, Min (1999): „Tradition“ und „Innovation“. Einblicke in vierzig Jahre Deutsch als Fremdsprache an chinesischen Hochschulen. In: Hans-Rüdiger Fluck und Jürgen Gerbig (Hg.): Spracherwerb Deutsch in Ost- und Zentralasien. Lehr- und Lerntraditionen im Wandel (1), S. 199-207.

Lin, Chunxia (2011): Warum geben Kinder in arme Familien ihre Chance für Weiterbildung auf? 寒门学子缘何放弃高考？. In: China Economie Times, 2011.

Liu, Runqing (2016): Research Methods in Forgeign Language Teaching. 外语教学 中的科研方法. 2. Aufl. Beijing: Foreign Language Teaching and Research Press.

Lohfert, Walter (1983): Kommunikative Spiele für Deutsch als Fremdsprache: Spielpläne u. Materialien für d. Grundstufe. 1. Aufl., 1. Dr. München: Hueber.

Loo, Angelika (2007): Teaching and learning modern languages in large classes. Zugl.: Berlin, Techn. Univ., Habil-Schr., 2007. Aachen: Shaker (Beiträge zur Didaktik).

Loo, Angelika (2012): Deutsch-Unterricht in großen Lerngruppen International. Ein Praxis-Hanbuch. Aachen: Shaker.

Loo, Angelika (2016): Unterricht mit großen Gruppen. In: Eva Burwitz-Melzer, Grit Mehlhorn, Claudia Riemer, Karl-Richard Bausch und Hans-Jürgen Krumm (Hg.): Handbuch Fremdsprachenunterricht. 6., völlig überarbeitete und erweiterte Auflage. Tübingen: A. Francke (UTB, 8043), S. 361-365.

Loo, Angelika (2017): Unterricht mit grossen Lerngruppen. In: Fremdsprache Deutsch (56), S. 3-9.

Luo, Anyuan (2001): Language Education in the Development of Western China. 西部大开发中的语言教育问题. In: Journal of The Central University for Nationalities(Humane and Social Sciences Edition 28 (3), S. 105-109.

Luo, Xiaomei (2012): Ist-Analyse der Ausbildung an Grundschule in unterentwickelten Regionen in Westchina. Am Beispiel von Grundschulen in Yunnan. 中国西部不发达地区小学英语教育的现状研究. In: Journal of Longdong University: Natural Science Edition 23 (1), S. 111-114. 
MacEdonia, Manuela (2007): Sprachspiele. Tipps \& Ideen zum Sprachenlernen. 5. Aufl. Linz: Veritas.

Macintyre, Peter D.; Dörnyei, Zoltán; Clément, Richard; Noels, Kimberly A. (1998): Conceptualizing Willingness to Communicate in a L2: A Situational Model of L2 Confidence and Affiliation. In: Modern Language Journal 82 (4), S. 545-562.

Maletzke, Gerhard (1996): Interkulturelle Kommunikation. Zur Interaktion zwischen Menschen verschiedener Kulturen. Opladen: Westdeutscher.

Mátyás, Emese (2009): Sprachlernspiele im DaF-Unterricht. Einblick in die Spielpraxis des finnischen und ungarischen Deutsch-als-FremdspracheUnterrichts in der gymnasialen Oberstufe sowie in die subjektiven Theorien der Lehrenden über den Einsatz von Sprachlernspielen. Dissertation. University of Jyväskylä. Online verfügbar unter https://jyx.jyu.fi/dspace/bitstream/ handle/123456789/21307/9789513936358.pdf?sequence=1, zuletzt geprüft am 12.12.2015.

Mátyás, Emese (2010): Sprachlernspiele: Ablauf und Ergebnisse eines Forschungsprojekts für die gymnasiale Oberstufe im finnischen und ungarischen DaF-Kontext. In: Informationen Deutsch als Fremdsprache 37, S. 383-400.

McCroskey, James C.; Richmond, Virginia P. (1990): Willingness to communicate: Differing cultural perspectives. In: Southern Communication Journal 56 (1), S. 72-77. DOI: 10.1080/10417949009372817.

McDonough, Jo (1994): A teacher looks at teachers' diaries. In: ELT Journal (48), S. 57-65.

Meng, Fanqiang; Chu, Shuai; Li, Qinghai (2017): Does the Expansion of Higher Education Alleviate the Urban-Rural Education Opportunity Inequality? 高等教育规模扩张是否缓解了城乡教育机会不平等?. In: Education and Economy 33 (4), S. 9-16.

Meyer, Hilbert (1994): Unterrichtsmethoden II Praxisband. 3. Aufl. Frankfurt (Main).

Ministerium für Bildung der VR China (2018): Die Anzahle der Hochschulen in China. 高等教育学校 (机构) 数. Online verfügbar unter http://www.moe. gov.cn/s78/A03/moe_560/jytjsj_2017/gd/201808/t20180808_344750.html, zuletzt geprüft am 20.04.2019.

Mitschian, Haymo (1991): Chinesische Lerngewohnheiten. Evaluierungen für den Deutsch-als-Fremdsprachenunterricht [in der Volksrepublik China]. Zugl.: Berlin, Techn. Univ., Diss., 1991. Frankfurt/Main: Verl. für Interkulturelle Kommunikation (Interdisziplinäre Studien zum Verhältnis von Migrationen, Ethnizität und gesellschaftlicher Multikulturalität, 4). 
Mitschian, Haymo (1999): Passivität asiatischer Lerner- Analyse einer Verhaltensbeschreibung. In: Hans-Rüdiger Fluck und Jürgen Gerbig (Hg.): Spracherwerb Deutsch in Ost- und Zentralasien. Lehr- und Lerntraditionen im Wandel (1), S. 45-60.

Niewalda, Katrin (2014): Sprachlernspiele - Aus Sicht der Lernenden in mehrfacher Hinsicht eine Effektive Unterrichtstechnik. In: Japanische Gesellschaft für Germanistik (Hg.): Neue Beiträge zur Germanistik, Bd. 13. München: Iudicium (13), S. 241-260.

Niewalda, Katrin (2015): Das Sprachlernspiel im Deutsch-als-FremdspracheUnterricht: Ergebnisse einer empirischen Untersuchung mit Lehrenden an japanischen Universitäten. In: 言語文化研究 34 (2), S. 127-162.

O'Connor, Kate Eliza (2008): "You choose to care": Teachers, emotions and professional identity. In: Teaching and teacher education 24 (1), S. 117-126. DOI: 10.1016/j.tate.2006.11.008.

Oerter, Rolf (1997): Psychologie des Spiels. 2., neu ausgestattete Aufl. Weinheim: Psychologie Verlags Union.

Pawlak, Mirosław; Mystkowska-Wiertelak, Anna; Bielak, Jakub (2015): Investigating the nature of classroom willingness to communicate (WTC): A micro-perspective. In: Language Teaching Research 20 (5), S. 654-671. DOI: $10.1177 / 1362168815609615$.

Peng, Jian-E; Woodrow, Lindy (2010): Willingness to Communicate in English: A Model in the Chinese EFL Classroom Context. In: language learning 60 (4). DOI: 10.1111/j.1467-9922.2010.00576.x.

Peng, Yue; Yang, Lei (2018): Optimizing the Teaching of German Quality Elective Course in Colleges in the Bachground of "One Belt, One Road": Taking German Teaching as a Research Example. ”一带一路”背景下高校非通用语 素质选修课教学优化探索. In: The Science Education Article Collects (415), S. 190-192.

Porsch, Raphaela (2014): Test. In: Jutta Settinieri (Hg.): Empirische Forschungsmethoden für Deutsch als Fremd- und Zweitsprache. Paderborn: Schöningh (UTB Sprachwissenschaft, 8541), S. 87-102.

Qi, Zihuan (2017): Ist-Analyse vom westchinesichen Bildungswesen. 中国西部 教育现状分析与研究. In: West China (3), S. 5.

Qin, Xiaoqing; Bi, Jing (2016): Quantitative Approaches ans Quantitative Data Analysis in L2 Research. 外语教学定量研究方法及数据分析. Beijing: Foreign Language Teaching and Research Press.

Raupach, Evelyne (2016): Möglichkeiten der Integration chinesischer Lernkulturen im fremdsprachlichen Deutschunterricht. In: Feldmeier, Alexis, Eichstaedt und Annett (Hg.): Lernkulturen-Schriftsprache in DaZ-Grammatik-Sprachliche Anforderungen in den Fächern. 41. Jahrestagung des 
Fachverbandes Deutsch als Fremdsprache und Zweitsprache an der Universität Münster 2014. Göttingen: Universitätsverlag Göttingen (Materialien Deutsch als Fremdsprache, 94), S. 53-62.

Reinbothe, Roswitha (2007): Sprachliche Schwierigkeiten im deutschen Unterricht in China. In: Ulrich Ammon, Roswitha Reinbothe und Jianhua Zhu (Hg.): Die deutsche Sprache in China. Geschichte, Gegenwart, Zukunftsperspektiven. München: Iudicium, S. 54-67.

Riemer, Claudia (2014): Forschungsmethodologie Deutsch als Fremd- und Zweitsprache. In: Jutta Settinieri (Hg.): Empirische Forschungsmethoden für Deutsch als Fremd- und Zweitsprache. Paderborn: Schöningh (UTB Sprachwissenschaft, 8541), S. 15-31.

Riemer, Claudia (2016): Affektive Faktoren. In: Eva Burwitz-Melzer, Grit Mehlhorn, Claudia Riemer, Karl-Richard Bausch und Hans-Jürgen Krumm (Hg.): Handbuch Fremdsprachenunterricht. 6., völlig überarbeitete und erweiterte Auflage. Tübingen: A. Francke (UTB, 8043), S. 266-271.

Roche, Jörg (2013): Fremdsprachenerwerb, Fremdsprachendidaktik. 3., vollständig überarbeitete Aufl. Tübingen, Basel: A. Francke (UTB, 2691: UTB basics).

Rösler, Dietmar (1994): Deutsch als Fremdsprache. Stuttgart: Metzler (Sammlung Metzler, Bd. 280).

Rösler, Dietmar (2015): Studienbegleitender Deutschunterricht in naturwissenschaftlich-technischen Studiengängen an Universitäten außerhalb des deutschsprachigen Raums. In: Zeitschrift für Interkulturellen Fremdsprachenunterricht 20 (1), S. 7-20.

Sánchez Benito, Juana; Sanz Oberberger, Carlos; Dreke, Michael (2007): Spielend Deutsch lernen. Interaktive Arbeitsblätter für Anfänger und Fortgeschrittene. [Nachdr.]. Berlin: Langenscheidt.

Scheuerl, Hans (1983): Die pädagogisch-anthropologische Dimension des Spiels. In: Karl Josef Kreuzer (Hg.): Handbuch der Spielpädagogik. 1. Aufl. 2 Bände. Düsseldorf: Schwann (Das Spiel unter pädagogischm, psychologischem und vergleichendem Aspekt, 1), S. 31-42.

Scheuerl, Hans (1991): Spiel- ein menschliches Grundverhalten? In: Hans Scheuerl (Hg.): Das Spiel. Theorien des Spiels. 10. Aufl. Weinheim: Beltz (Berlz Studienbuch, Bd. 2), S. 189-208.

Scheuerl, Hans (1994): Das Spiel. 12., unveränd. Aufl. Weinheim: Beltz.

Schiffler, Ludger (1985): Interaktiver Fremdsprachenunterricht. 2., korr. Aufl. Stuttgart.

Schiller, Friedrich (1991): Spiel und Freiheit- der ästhetische Zustand. In: Hans Scheuerl (Hg.): Das Spiel. Theorien des Spiels. 10. Aufl. Weinheim: Beltz (Berlz Studienbuch, Bd. 2), S. 34-40. 
Schmidjell, Annegret (2017): Interaktionsorientierter Unterricht. Lernende in großen Gruppen aktivieren. In: Fremdsprache Deutsch (56), S. 10-18.

Schweckendiek, Jürgen (2001): Spiele und Spielerisches. Zur Förderung der Gruppenintergration und zur Binnendifferenzierung. In: Fremdsprache Deutsch (25), S. 9-19.

Schwerdtfeger, Inge Christine (1989): Arbeits- und Übungsformen: Überblick. In: Karl-Richard Bausch, Herbert Christ und Hans-Jürgen Krumm (Hg.): Handbuch Fremdsprachenunterricht. Tübingen: Francke; UTB (UTB), S. $187-190$.

Schwerdtfeger, Inge Christine (2007): Gruppenunterricht und Partnerarbeit. In: Karl-Richard Bausch (Hg.): Handbuch Fremdsprachenunterricht. 5. Aufl. Tübingen: Francke (UTB Pädagogik, Sprachwissenschaften, 8042/8043), S. 254-257.

Settinieri, Julia (2012): Fokus Datenanalyse: Statitische Verfahren Grundlagenbeitrag. In: Sabine Doff (Hg.): Fremdsprachenunterricht empirisch erforschen. 1. Aufl. Tübingen: Narr, S. 249-270.

Smith, Lesly; King, Jim (2018): Silence in the foreign language classroom: the emotional challenges for L2 teachers. In: Agudo, Juan de Dios Martínez (Hg.): Emotions in Second Language Teaching. Theory, Research and Teacher Education. Cham: Springer International Publishing, 323-339.

Spitzer, Manfred (2000): Geist im Netz. Modelle für Lernen, Denken und Handeln. Heidelberg: Spektrum Akademischer Verlag.

Stadler-Altmann, Ulrike (2015): Gebaute Umgebung als Lernumgebung: Haben Schulgebäude und Klassenzimmer Einfluss auf Lehren und Lernen? In: Ulrike Stadler-Altmann (Hg.): Lernumgebungen. Erziehungswissenschaftliche und architekturkritische Perspektiven auf Schulgebäude und Klassenzimmer, S. 49-68.

Steinhilber, Jürgen (1979): Zur Didaktik des Unterrichtsspiels im Fremdsprachenunterricht. Berlin, Freie Univ., Diss., 1979.

Steinhilber, Jürgen (1986): Didaktik des Spiels im Fremdsprachenunterricht. Zugl.: Univ., Diss., u.d.T.: Zur Didaktik des Unterrichtsspiels im Fremdsprachenunterricht. 5. Aufl. Frankfurt am Main: Hirschgraben-Verl.

Steinmüller, Ulrich (2019): Konfuzius oder Nürnberger Trichter. Überlegungen zur Didaktik und Methodik des DaF-Unterrichts in China. In: Informationen Deutsch als Fremdsprache 46 (2), S. 227-238.

Stellfeld, Elke (1995): Zu Schreibspielen als Sprachlernspiele im Fremdsprachenunterricht des mittleren Schualters (Russischunterricht). Dissertation. Ottovon-Guericke-Univeristät Magdeburg, Magdeburg. 
Stork, Antje; Zhao, Jin (2009): Schweigsame chinesische Seminarteilnehmer: Angst vor Gesichtsverlust? In: Zielsprache Deutsch 36 (2), S. 31-49.

Technische Universität Kunming (23.08.2018): Über die Arbeit von Immatrikulation im Jahr 2018 der Technischen Universität Kunming. 昆明理工大学2018 年本科招生工作取得新突破. Online verfügbar unter http://www.kmust. edu.cn/html/xyxw/2018/08/23/4f6b60e7-e1af-4c35-bc27-28fac0a9cf59.html, zuletzt geprüft am 20.04.2019.

Technische Universität Kunming (30.10.2018): Profil und Geschichte der Technischen Universität Kunming. 学校简介. Online verfügbar unter http://www. kmust.edu.cn/html/xxgk/xxjj/1.html, zuletzt geprüft am 27.02.2019.

Thelen, Gabriele (2003): Chinesische Germanistikstudierende zwischen gestern und heute. eine empirische Studie. Regensburg (Materialien Deutsch als Fremdsprache, 67).

Tian, Chunyu (2017): Didaktische und methodische Aspekte des Lehrens und Lernens im DaF-Unterricht für Studierende in naturwissenschaftlichen und technischen Fachgebieten in China: am Beispiel des DaF-Unterrichts an der Chinesisch-Deutschen Technischen Fakultät der Qingdao University of Science and Technology. München: Iudicium Verlag.

van der Kooij, Rimmer (1983): Die psychologischen Theorien des Spiels. In: Karl Josef Kreuzer (Hg.): Handbuch der Spielpädagogik. 1. Aufl. 2 Bände. Düsseldorf: Schwann (Das Spiel unter pädagogischm, psychologischem und vergleichendem Aspekt, 1), S. 297-335.

Vollmer, Helmut J. (2007): Leistungsmessung, Lernerfolgskontrolle, Selbstbeurteilung: Überblick. In: Karl-Richard Bausch (Hg.): Handbuch Fremdsprachenunterricht. 5. Aufl. Tübingen: Francke (UTB Pädagogik, Sprachwissenschaften, 8042/8043), S. 365-370.

Wang, Bo (2013): Ist-Analyse der Beziehung von Bildungsresourcen und Einkommen: Ein Vergleich zwischen Ost- und Westchina. 我国东部、西部教 育资源与居民收入的分配现状及其相互作用. In: Distance education in China (12), S. 52-54.

Wang, Jing; Yang, Nan (2017): A Survey of the Current Rural Basic Education in Yunnan Border Areas and Policy Suggestions. 云南边境地区农村基础教育 发展状况与政策建议. In: Academic Expoloration (7), S. 141-145.

Wang, Min (1988): Hauptprobleme der Deutschlernenden aus China mit der deutschen Aussprache. In: Informationen Deutsch als Fremdsprache 15 (1), S. 76-82.

Wang, Qiang; Zhang, Ning (2011): Teaching in Large Classes- English as Foreign Language. Online verfügbar unter https://warwick.ac.uk/fac/soc/al/research/ groups/llta/resources/telc/5._wang_qiang_overview_of_china_research_0. pdf, zuletzt geprüft am 10.04.2018. 
Wang, Yingpin (2007): Mündliche kommunikative Fähigkeiten chinesischer Deutschlerner. Probleme und Perspektive. München: Iudicium.

Warwitz, Siegbert A.; Rudolf, Anita (2004): Vom Sinn des Spielens. Reflexionen und Spielideen. 2., aktualisierte Aufl. Baltmannsweiler: Schneider Hohengehren.

Wei, Ying (2015): The Empirical Research on Employment Intentions of Ordinary College Graduates in Yunnan Pronvince. Dissertation. Huazhong University of Science and Techonlogy, Wuhan.

Wei, Yumei; Liu, Xianchun (2016): Zur Verbesserung der Bildungsgleichheit von Bildungschancen an Hochschulen in Westchina. 全面小康社会建设：西部 高等教育入学机会公平的提升. In: Jiangsu Journal of Higher Education (2), S. $78-80$.

Wei, Yuqing (2009): Entwicklung des Fachs: Germanstik. 德语专业教育发展研 究. In: Weidong Dai und Wenzhong Hu (Hg.): Entwicklung der chinesischen Fremdsprachenbildungswesen (1949-2009). 中国外语教育发展研究 (19492009). Shanghai: Shanghai Foreign Language Education Press, S. 270-307.

Wei, Yuqing; Qin, Wenwen (2017): Geschichte, Entwicklung und Zukunft der chinesischen Germanistik. In: Jahrbuch für Internationale Germanistik 49 (2), S. $145-156$.

Wen, W. P.; Clément, Richard (2003): A Chinese Conceptualisation of Willingness to Communicate in ESL. In: Language, Culture and Curriculum 16 (1), S. 18-38. DOI: 10.1080/079083103086666654.

Winkler, Markus (2011): Arbeiten und Unterricht mit Großgruppen für DaF. Goethe-Insitut Rabat/ Casablanca. Online verfügbar unter http://www.goethe.de/ins/ma/rab/pro/pasch/dok/dok.pdf, zuletzt geprüft am 10.04.2018.

Xie, Xiaoyan (2010): Why are students quiet? Looking at the Chinese context and beyond. In: ELT Journal 64 (1), S. 10-20. DOI: 10.1093/elt/ccp060.

Xu, Fei (2017): Research on the Higher-Education Reform of Foreign Language in the Background of " One Belt and One Road " .“一带一路”背景下外语 高等教育改革研究. In: Theory and Practice of Education 37 (12), S. 45-46.

Yang, Jianpei; Loo, Angelika (2007): Vom Notfall zur Innovation? Zur Großgruppendidaktik im chinesischen DaF-Unterricht. In: German as foreign language (3), S. 73-90. Online verfügbar unter http://www.gfl-journal.de/32007/yang_loo.pdf.

Yang, Mengying (2017): Effektivität von Vokabellernstrategien im DaF-Unterriht in China. Dissertartion. Phillip- Universität, Marburg. Online verfügbar unter https://archiv.ub.uni-marburg.de/diss/z2017/0775/pdf/dmy.pdf, zuletzt geprüft am 14.02.2019.

Yeh, Lien Chuan (2014): Gezielte Vermittlung und Akzeptanz von Wortschatzlernmethoden: Eine empirische Untersuchung über den Einfluss von 
Lehrmethoden auf das Lernverhalten bei DaF-Studierenden in Taiwan und in China. Marburg: Tectum Verlag.

Yin, Hong-biao; Lee, John Chi-Kin (2012): Be passionate, but be rational as well: Emotional rules for Chinese teachers' work. In: Teaching and teacher education 28 (1), S. 56-65.

You, Chenjing; Dörnyei, Zoltán (2016): Language Learning Motivation in China. Results of a Large-Scale Stratified Survey. In: Applied Linguistics 37 (4), S. $495-$ 519. DOI: 10.1093/applin/amu046.

Zarrinabadi, Nourollah (2014): Communicating in a second language: Investigating the effect of teacher on learners' willingness to communicate. In: System 42 (1), S. 288-295.

Zarrinabadi, Nourollah; Tanbakooei, Nooshin (2016): Willingness to Communicate: Rise, Development, and Some Future Directions 10 (1). DOI: 10.1111/ $\operatorname{lnc} 3.12176$.

Zeilinger, Miriam (2006): Beratung von ostasiatischen Studierenden. In: Zeitschrift für Interkulturellen Fremdsprachenunterricht 11 (2), S. 21-23. Online verfügbar unter http://www.ualberta.ca/ german/ejournal/Zeilinger1.htm, zuletzt geprüft am 11.05.2015.

Zhang, Jiayi; Beckmann, Nadin; Beckmann, Jens F. (2018): To talk or not to talk: A review of situational antecedents of willingness to communicate in the second language classroom. In: System 72, S. 226-239. DOI: 10.1016/j. system.2018.01.003.

Zhang, Xiaoping; Zhang, Liang (2015): Zulassungpriorität von hochqualifizierter chinesischen Universitäten. Am Beispiel von “211 Hochschulen”. 中国高 质量大学入学机会和招生偏好研究. In: Journal of Higher Education (7), S. 28-35.

Zhao, Junhai; Zhang, Biao (2017): A Study on the Demand and Training Model for Localized Foreign Language Interpreters and Translators in Yunnan. 云南 本土特色外语翻译专业人才需求及培养模式研究. In: Education and Teaching Research 31 (3), 38-43,115.

Zhao, Xinguo; Mao, Xiaoling (2012): Probleme der Enwicklung der Grundausbildung für Minderheitsnationen in Provinz Yunnan und deren Ursachen. 现 阶段云南发展民族基础教育面临的问题及原因探析. In: Journal of Southwest Univeristy for Nationalities 33 (8), S. 221-224.

Zhao, Zhiyong (2019): Deutschdidaktik und -methodik in China - Entwicklungen und Erfahrungen. In: Informationen Deutsch als Fremdsprache 46 (1), S. 19-39.

Zheng, Mingyuan (2018): Reflections on Foreign Language Educational Policy Adjustments in China from Perspective of "One Belt And One Road" 
Construction. “一带一路” 视域下中国外语教育政策调整问题的若干思 考. In: Heilongjiang Researches on Higher Education (5), S. 47-50.

Zhu, Jianhua (2003): Deutsch als Anwendungsfach für Chinesen. Probleme und Perspektiven. In: Ulrich Wannagat (Hg.): Deutsch als zweite Fremdsprache in Ostasien - neue Perspektiven. [... internationales Symposium ... vom 27. - 30. April 1999 am Chung Chi College der Chinesischen Universität von Hongkong]. Tübingen: Stauffenburg (Arbeiten zur angewandten Linguistik, 2), S. 35-42.

Zhu, Jianhua (2007a): „Hochschuldeutsch“- Deutsch als Anwendungsfach an Hochschulen und Univeristäten. In: Ulrich Ammon, Roswitha Reinbothe und Jianhua Zhu (Hg.): Die deutsche Sprache in China: Geschichte, Gegenwart, Zukunftsperspektiven, S. 141-152.

Zhu, Jianhua (2007b): Neue Orientierungen für Deutsch als Anwendungsfach in China. In: Neue Beiträge zur Germanistik 134, S. 91-102.

Zhu, Jianhua (2009): Entwicklung des Fachs: Hochschuldeutsch. 大学德语教 育发展研究. In: Weidong Dai und Wenzhong Hu (Hg.): Entwicklung der chinesischen Fremdsprachenbildungswesen (1949-2009). 中国外语教育发 展研究 (1949-2009). Shanghai: Shanghai Foreign Language Education Press, S. 628-654.

Zhu, Jianhua (2019): Perspektiven zur Didaktik und Methodik für Deutsch als Fremdsprachen (DaF) in China - besonders am Besipiel „Hochschuldeutsch“. In: Informationen Deutsch als Fremdsprache 46 (1), S. 7-18.

Zhu, Jianhua; Li, Yuan (2004): Lehrwerksentwicklung für Deutsch als Anwendungsfach in China. Am Beispiel „Klick auf Deutsch“. In: Hans-Werner Hess (Hg.): Didaktische Reflexionen. „Berliner Didaktik“ und Deutsch als Fremdsprache heute. Tübingen: Stauffenburg (Arbeiten zur angewandten Linguistik, 3), 97-107.

Zhu, Jianhua; Li, Yuan (2011): Lehrerhandbuch für Klick auf Deutsch. Beijing: Foreign Language Teaching and Research Press.

Zhu, Jianhua; Li, Yuan (2013): Lehrwerk „Klick auf Deutsch“. 2. Aufl. 4 Bände. Beijing: Verlag für Foreign Language Teaching and Research (1).

Ziebell, Barbara; Meese, Herrad (2004): Unterrichtsbeobachtung und Lehrerverhalten. 3. [Dr.]. Berlin [u.a.]: Langenscheidt (Fernstudienprojekt zur Fort- und Weiterbildung im Bereich Germanistik und Deutsch als Fremdsprache: Teilbereich Deutsch als Fremdsprache).

Dieses Werk enthält zusätzliche Informationen als Anhang. Sie können von unserer Website heruntergeladen werden: http://dx.doi.org/10.3726/b16557 Dazu müssen Sie den folgenden Freischaltcode eingeben: PL20Dx37H 


\section{Deutsch als Fremdsprache in der Diskussion}

Herausgegeben von Martin Löschmann

Band 1 Martin Löschmann: Effiziente Wortschatzarbeit. Alte und neue Wege. Arbeit am Wortschatz. 1993.

Band 2 Manfred Pudszuhn: Fachunterricht versus Sprachunterricht. Untersuchungen zum studienvorbereitenden Fachsprachunterricht (DaF) für Ausländer. 1994.

Band 3 Martin Hahn / Sebastian Künzel / Gerhard Wazel (Hrsg.): Multimedia - eine neue Herausforderung für den Fremdsprachenunterricht. 1996. 2., durchgesehene Auflage 1998.

Band 4 Martin Löschmann/Magda Stroinska (Hrsg.): Stereotype im Fremdsprachenunterricht. 1998.

Band 5 Gerhard Wazel/Institut für Interkulturelle Kommunikation e.V. (Hrsg.): Interkulturelle Kommunikation in Wirtschaft und Fremdsprachenunterricht. 2001.

Band 6 Dagmar Blei: Zur Fachgeschichte Deutsch als Fremdsprache. Eigengeschichten zur Wissenschaftsgeschichte. 2003.

Band 7 Martin Hahn/Gerhard Wazel (Hrsg.): Theorie und Praxis des DaF- und DaZ-Unterrichts heute. 20 Jahre Institut für Interkulturelle Kommunikation e.V. 2011.

Band 8 Vlasta Kučiš (Hrsg.): Translation in Theorie und Praxis. 2013.

Band 9 Ellen Tichy / Viktoria llse (Hrsg.): Deutsch in Mittelosteuropa nach 1989. 25 Jahre Germanistikstudiengänge, Deutschlehrerausbildung, DaF-Lehrwerke und DaF-Unterricht. 2014.

Band 10 Martin Löschmann (Hrsg.): Humor im Fremdsprachenunterricht. 2015.

Band 11 Janek Scholz / Marvin Wassermann / Johanna Zahn: DaZ-Unterricht an Schulen. Didaktische Grundlagen und methodische Zugänge. 2020.

\section{DaZ und DaF in der Diskussion}

Herausgegeben von Jörg Meier, Beatrice Müller und Thorsten Roelcke

Band 12 Jing Zeng: Sprachlernspiele im DaF-Unterricht in der VR China. Möglichkeiten und Herausforderungen ihres Einsatzes in Universitäten. 2020.

www.peterlang.com 
